\title{
HSB 84A Pumping Test
}

by

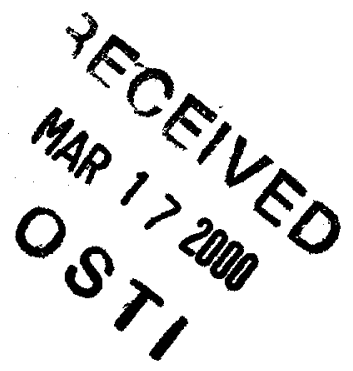

W. Maloney

Westinghouse Savannah River Company

Savannah River Site

Aiken, South Carolina 29808

This paper was prepared in connection with work done under the above contract number with the U.S. Department of Energy. By acceptance of this paper, the publisher and/or recipient acknowledges the U.S. Government's right to retain a nonexclusive, royalty-free license in and to any copyright covering this paper, along with the right to reproduce and to authorize others to reproduce all or part of the copyrighted paper. 


\section{DISCLAIMER}

This report was prepared as an account of work sponsored by an agency of the United States Government. Neither the United States Government nor any agency thereof, nor any of their employees, makes any warranty, express or implied, or assumes any legal liability or responsibility for the accuracy, completeness, or usefulness of any information, apparatus, product or process disclosed, or represents that its use would not infringe privately owned rights. Reference herein to any specific commercial product, process or service by trade name, trademark, manufacturer, or otherwise does not necessarily constitute or imply its endorsement, recommendation, or favoring by the United States Government or any agency thereof. The views and opinions of authors expressed herein do not necessarily state or reflect those of the United States Government or any agency thereof.

This report has been reproduced directly from the best available copy.

Available for sale to the public, in paper, from: U.S. Department of Commerce, National Technical Information Service, 5285 Port Royal Road, Springfield, VA 22161

phone: (800) 553-6847

fax: (703) 605-6900

email: orders@ntis.fedworld.gov

online ordering: http://www.ntis.gov/ordering.htm

Available electronically at http://www.doe.gov/bridge

Available for a processing fee to U.S. Department of Energy and its contractors, in paper, from: U.S. Department of Energy, Office of Scientific and Technical Information, P.O. Box 62, Oak Ridge, TN 37831-0062

phone: (865)576-8401

fax: (865)576-5728

email: reports@adonis.osti.gov 


\section{DISCLAIMER}

Portions of this document may be illegible in electronic image products. Images are produced from the best available original document. 


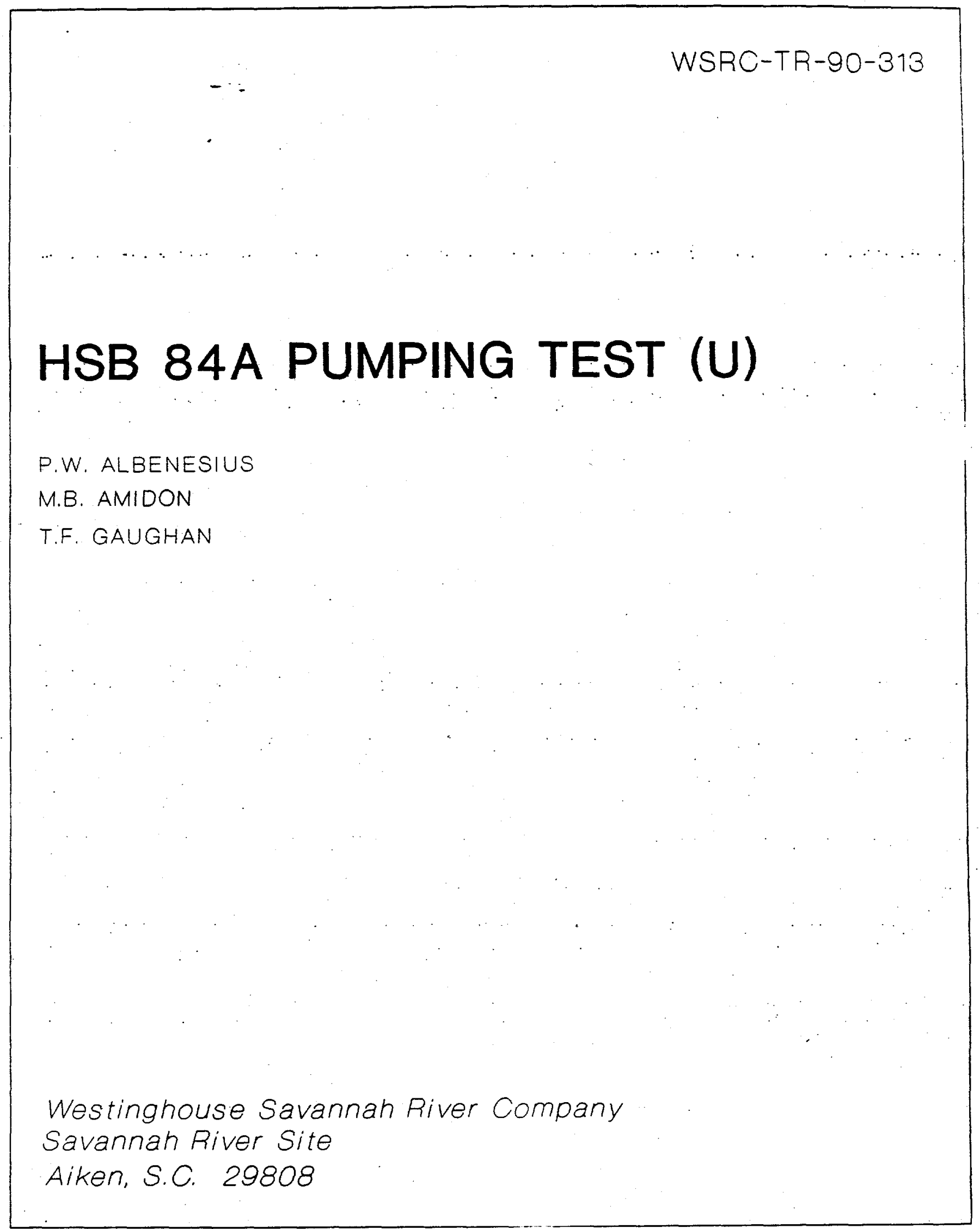


HSB 84 A PUMPING TEST ( 0 )

P.W. ALBENESIUS

M.A. AMIDON

T.F. GAUGHAN

Publication Date: August 15, 1990

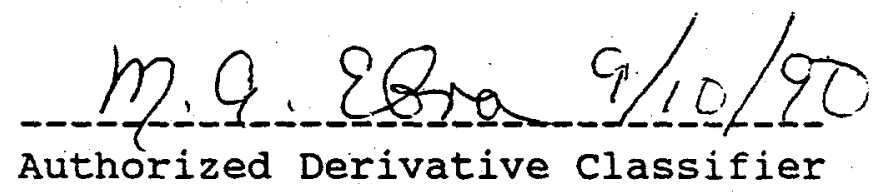

Westinghouse Savannah River Company Savannah River Site

Aiken, S.C. 29808 
Two constant discinarge, muiciple weli pumping tests were performed in the Congaree aquifer at the $\mathrm{H}$-Area seepage basins duing the weeks of April 30 through May 11. The purpose of the tests. was to collect information that might determine the source of groundwater contamination in the Congaree aquifer and to estimate the hydraulic parameters of the aquifer.

Transmissivity estimates from data collected in Test one ranged from $1644 \mathrm{ft}^{2} /$ day to $2253 \mathrm{ft}^{2} /$ day with an average of $2013 \cdot \mathrm{ft}^{2} /$ day and from $181.2 \mathrm{ft}^{2} /$ day to $2562 \mathrm{ft}^{2} /$ day with an average of $2269 \mathrm{ft}^{2} / \mathrm{day}$ in Test Two. Some leakage through the confining bed was apparent in the vicinity of observation well HSB 69A.

This report includes the data collected, the analyses, results and interpretation of the pumping tests performed at HSB 84A. It should serse as a good baseline for future studies on the subject of contaminant migration in the Congaree aquifer on the Savannah River site. 
CONTENT'

INTRODDCTION

DIscussION

Project Background

Pre-Test. Trend Data

Drawdown and Recovery Data

Test one

Test Two

Leakage at HSB 69A

other Results

sampling

RECOMMENDATIONS FOR FUTURE WORR

CONCLUSIONS

REFERENCES

APPENDIX A

APPENDIX B

APPENDIX $C$

APPENDIX D

APPENDIX E

APPENDIX $\mathbf{F}$

APPENDIX G
Test one Drawdown Data

Test one Aqtesolv Plots

Test Two Drawdown and Recovery Data

Test Two Drawdown Plots

Test Two Recovery Plots.

Chemical Data

Pre-Test Water Level Data 


\section{LIST OF FIGURES}

1. Pumping Well and Observation Wells

2. Test one Barometric Pressure

3. Test Two Barometric Pressure

4. Test one Pumping Rate

5. Test Two Pumping Rate

6. Sectional View of Congaree Aquifer.

7. Tritium Concentrations

\section{IIST OF TABIESS}

1. Test one Analytical Results 


\section{INTRODOCTION}

Two constant discharge, multiple well pumping tests were performed in the Congaree Aquifer at the $\mathrm{H}$-area seepage basins during the weeks of April 30 through May 11. Monitoring well HSB $84 \mathrm{~A}$ was used as the pumping well and wells HSB 84B,C and D, HSB 69 and 69A, HSB 68A, HSB 139A, HSB $83 \mathrm{~A}, \mathrm{HSB} 86 \mathrm{~A}$, and HSB $118 \mathrm{~A}$ were used as observation wells in both" tests, (see Figure 1 for location map)... The. first test, hereafter referred to as Test one, was conducted on May. 1 and May 2. The average pumping rate for Test one was fifty nine gallons per minute and the duration of pumping was approximately twenty two hours. The test ended due to equipment problems. Because the exact time of pumping cessation was unknown, recovery in the observation wells was not recorded. A second test (Test Two) was conducted on May 8 and May 9 at an average pumping rate of fifty seven gallons per minute for a twenty one and a half hour duration. Recovery was measured in the observation wells for a twenty four hour period after pumping ceased for Test Two.

Water samples were collected every two hours from the pumping well during both tests and analyzed for tritium by an on site laboratory. Results from both tests were plotted and included in this report. During Test Two continuous measurements of $\mathrm{pH}$, Specific conductance, Temperature, dissolved oxygen and redox were taken from the discharging well: Those results will be included in a later report.

Several analytical solutions were used to reduce the drawdown and recovery data from both tests. The field measurements and the data reduction are included along with the results and conclusions.

\section{DISCUSSION}

\section{PROJECT BACKGROUND}

Since its installation in 1985, monitoring well HSB $84 \mathrm{~A}$ has been the only Congaree well in the General Separations Area to display levels of tritium in excess of 10,000 $\mathrm{pCi} / \mathrm{ml}$. other congaree wells in the area display tritium contamination at levels less than $200 \mathrm{pCi} / \mathrm{ml}$. Monitoring data indicate that contaminated water from the shallower water table and McBean hydrologic units entered the congaree through a localized zone of high leakance near HSB 84A. Several theories about the nature and location of the hydraulic connection between the congaree and shallower units have been postulated. A pumping test at HSB $84 \mathrm{~A}$ was initiated to gain information about aquifer characteristics 

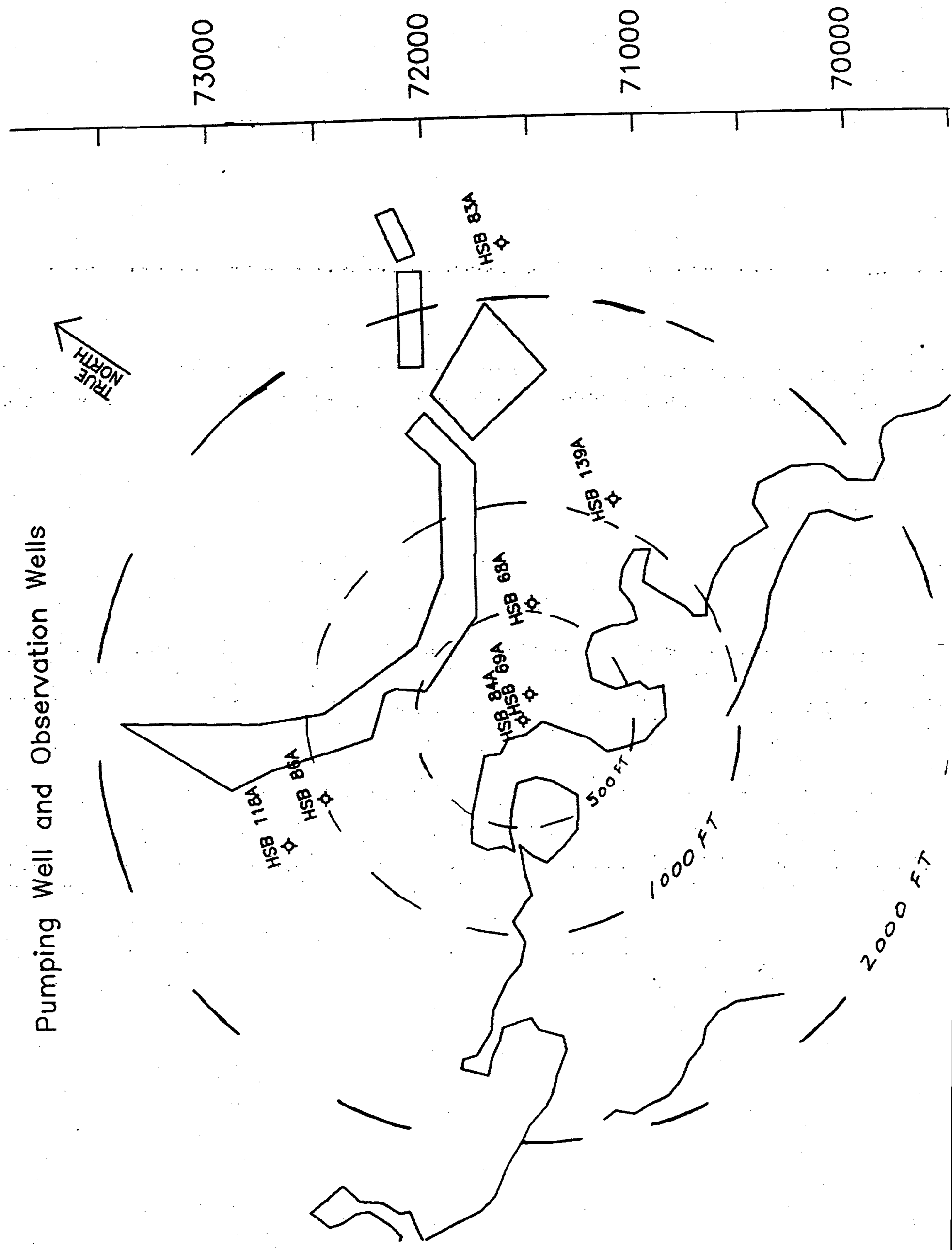
and leakance values in the vicinity of the anomolous congaree- contamination.

\section{PRE-TES'I TREND DATE}

Water levels were continuously recorded from April 27 until April 30 in all of the wells in the HSB 84 and 69 clusters. After the completion of Test one, water levels were continuously recorded in the same wells from May. 2. through May 6, prior to the start of Test Two. The data were recorded using pressure transducers and a Hermit TM 2000B data logger.

Barometric data were obtained from the SRL meteorology lab for the time period that corresponded to both the pretest water levels and the two pumping tests. In addition; readings were taken every hour during both tests using a field barometer and that data is plotted and included as Figures 2 and 3 . An attempt was made to compute the barometric efficiency of the congaree aquifer using the pretest data but barometric changes were so small during the period of data aquisition that it was not possible to discern any effect on water levels resulting from barometric pressure changes. Changes in barometric pressure during the drawdown portions of both tests were small, but during the recovery portion of Test Two, the barometric pressure made a discernible drop. Because hydraulic parameter estimates using the recovery data were in good agreement with estimates based on drawdown data, the drop in barometric pressure was not interpreted as having any effect on water levels. This is in contrast to the 1988. Congaree pumping test conducted by $\mathrm{CH}_{2} \mathrm{M}$ Hill in which barometric efficiency was estimated to be about $25 \%$ for the Congaree aquifer ${ }^{1}$.

\section{DRAWDOWN AND RECOVERY DATA}

Test one: Test one was conducted for an approximately 22 hour period at a constant discharge rate of 59 gallons per minute. The pumping rate was verified every hour and a graph depicting pumping rate through time is included as Figure 4. Drawdown was measured using pressure transducers and a data logger for wells HSB $84 \mathrm{~A}, 84 \mathrm{~B}, 84 \mathrm{C}, 84 \mathrm{D}, 69 \mathrm{~A}$, and 69 , throughout the test. Drawdown was measured using an electric water level tape in wells HSB $86 \mathrm{~A}, 118 \mathrm{~A}, 68 \mathrm{~A}, 139 \mathrm{~A}$ and $83 \mathrm{~A}$. Because of problems with the generator (power source for the pump), the exact time that pumping ceased is not known and therefore it was not possible to record the recovery of water levels for Test one. The drawdown data were analyzed by hand plotting using the cooper Jacob straight Iine approximation and the Theis non-equilibrium solution, and by computer software using the Theis nonequilibrium solution and the Hantush Jacob leaky confined. 


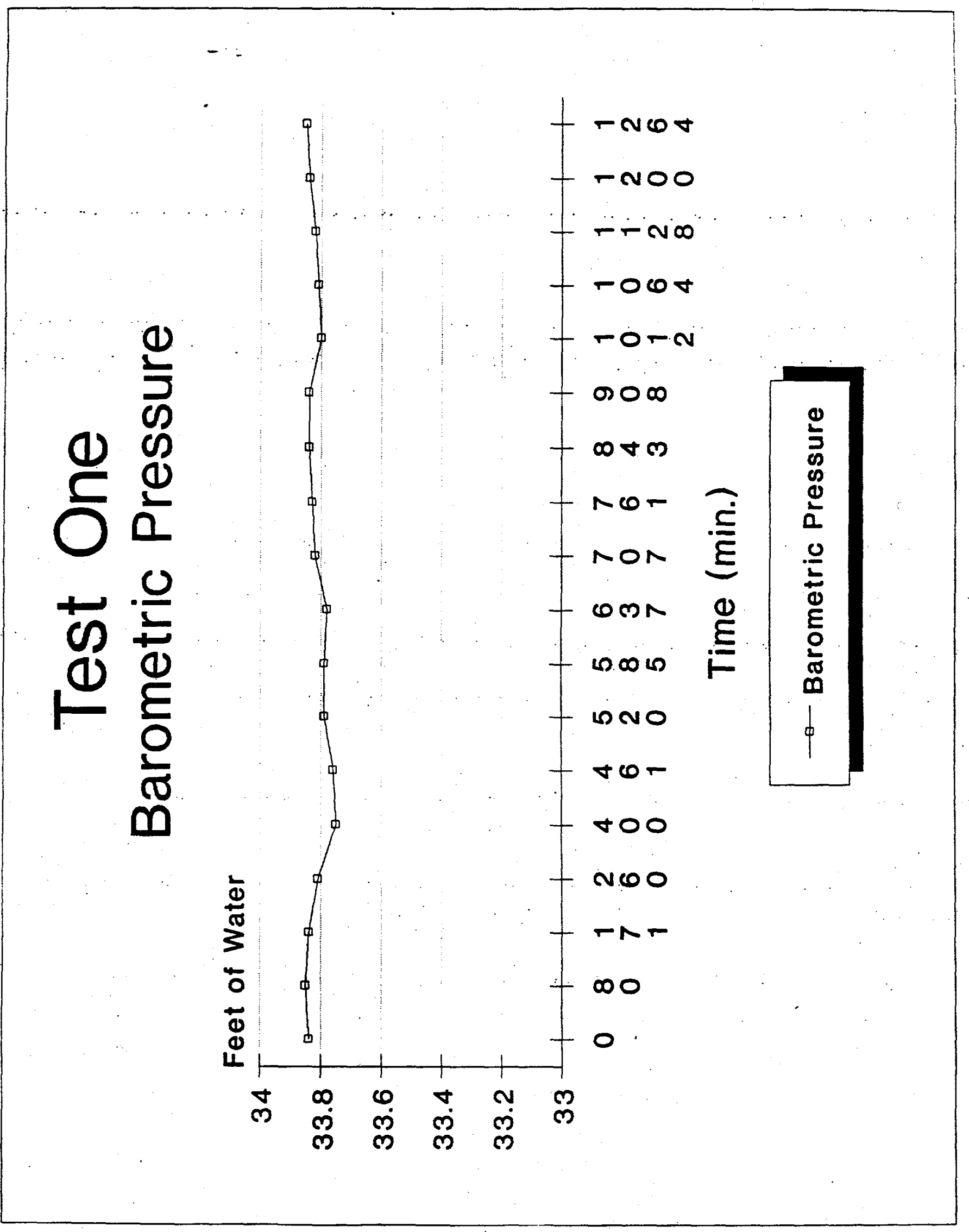




\section{Test Two Barometric Pressure}

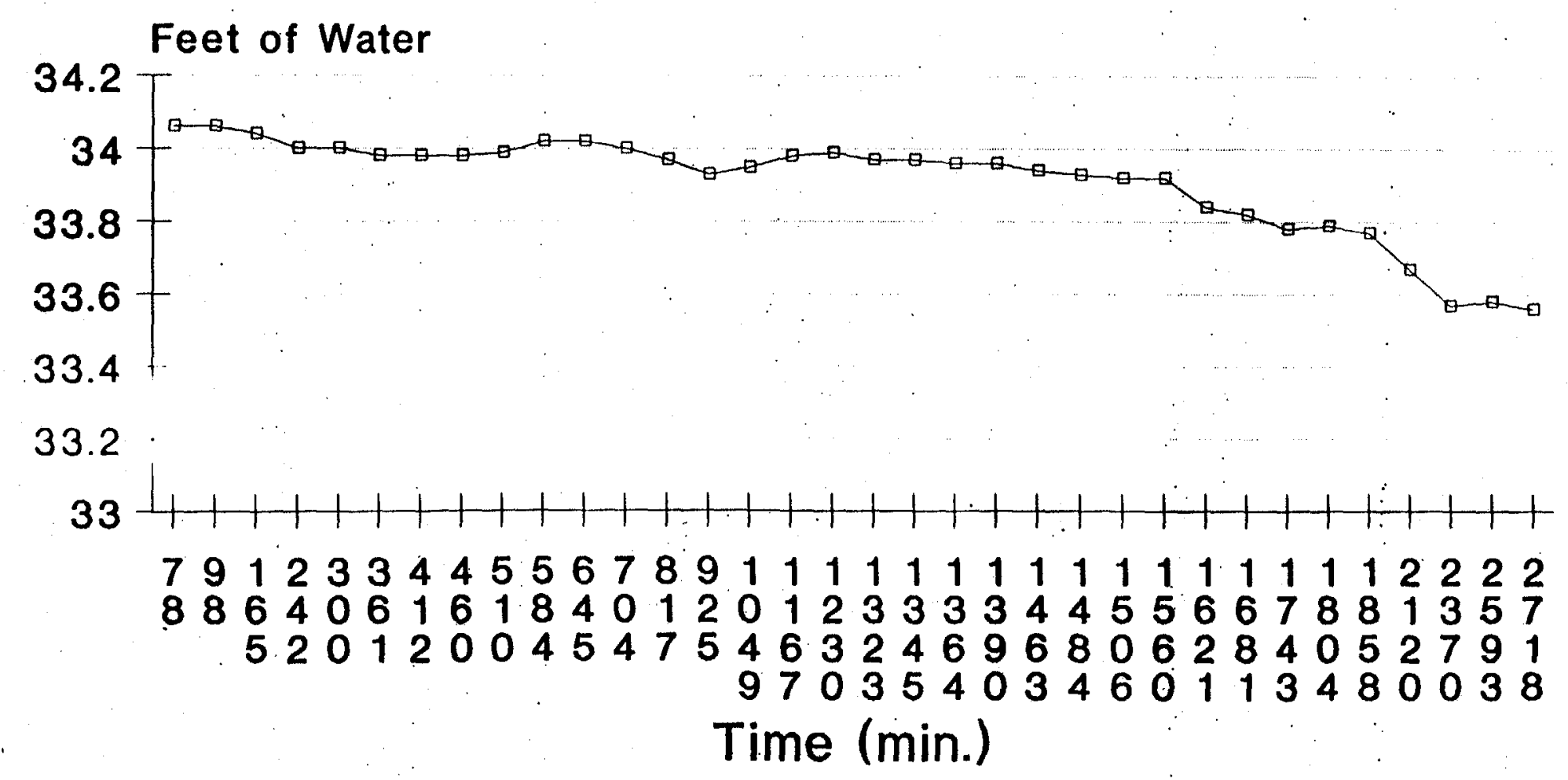

$\rightarrow$ Barometric Pressure 
aquifer solution. Results from these solutions are included as Table 1 .

Test Two: Test Two was conducted at a constant discharge rate of 57 gallons per minute for a 21.5 hour duration. Monitoring of drawdown was done for the same wells and in the same manner as in Test one. Recovery data were recorded in hand measured wells for a 24 hour period after pumping ceased and for a 96 hour period in the instrumented wells. The same analytical solutions that were applied in Test one were also applied in Test Two. Recovery data were analyzed using a computer program based on the Theis non-equilibrium solution. Fluctuations in pumping rate for Test Two were much greater than in Test one (Figures 4 and 5), and in the closest observation well (HSB 69A), the drawdown seemed to be affected by the variable pumping rate. The other observation wells did not seem to display effects of non-constant discharge, probably related to their greater distances from the pumping well. Results from the analyses of both the drawdown and recovery portions of the test are included as Table 2 .

Several analytical solutions were used to estimate the hydraulic parameters of the congaree Aquifer. The results are tabulated in Tables 1 and 2 of this report. Solutions used were the Cooper Jacob straight line approximation the Theis non-equilibrium solution, (as described in Heath ${ }^{2}$ ), the Theis recovery solution, and the Hantush Jacob leaky solution, (as applied in Aqtesolv ${ }^{3}$ and described in Hantush Jacob, 1958). Plots were done by hand and by computer and compared for consistency. The Theis non-equilibrium solution was chosen as the most applicable for this particular set of tests.

The Cooper Jacob straight line solution is based on the cone of depression assuming a steady shape. The steady shape is related to distance from the pumping well and total time of pumping. The greater the distance from the pumping well, the longer it takes to achieve steady shape of the cone. For that reason, the confidence level of estimates obtained using the Cooper Jacob method, diminishes with distance from the pumping well: Observation well HSB 83A was at the greatest distance from the pumping well (2253 feet) and as a result, displayed the least amount of drawdown. The hydraulic parameters estimated for $83 \mathrm{~A}$ using the straight line approximation are significantly different than those estimated from the other wells. However, using the Theis non-equilibrium solution, which does not depend on steady state or steady shape of the drawdown cone, the estimated parameters for the aquifer at HSB $83 \mathrm{~A}$ are very similar to those estimated at other observation wells, (see Tables 1 and 2 ). 


\section{Test One Pumping Rate}

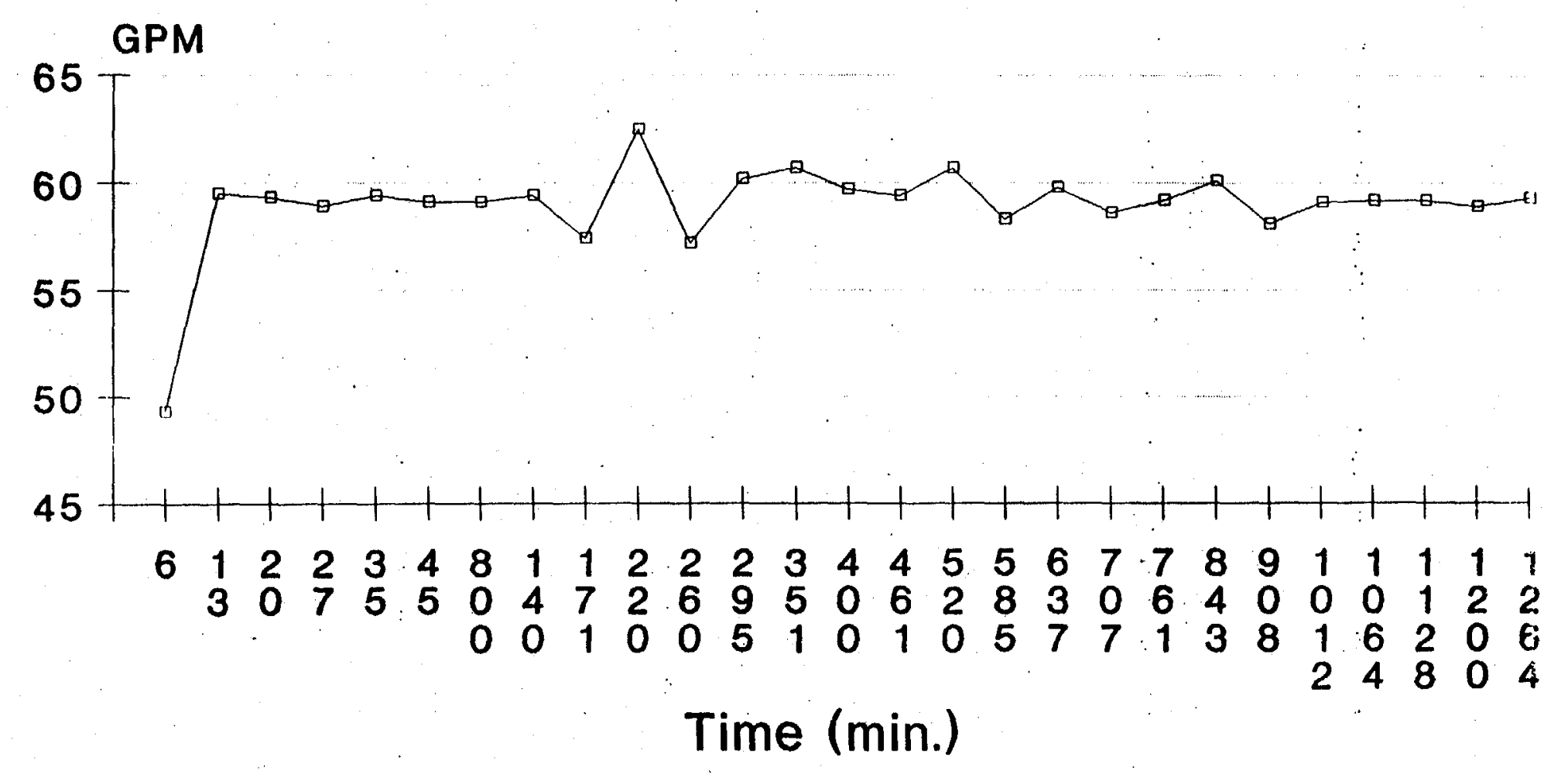

May $1-2$ 


\section{Test Two Pumping Rate}

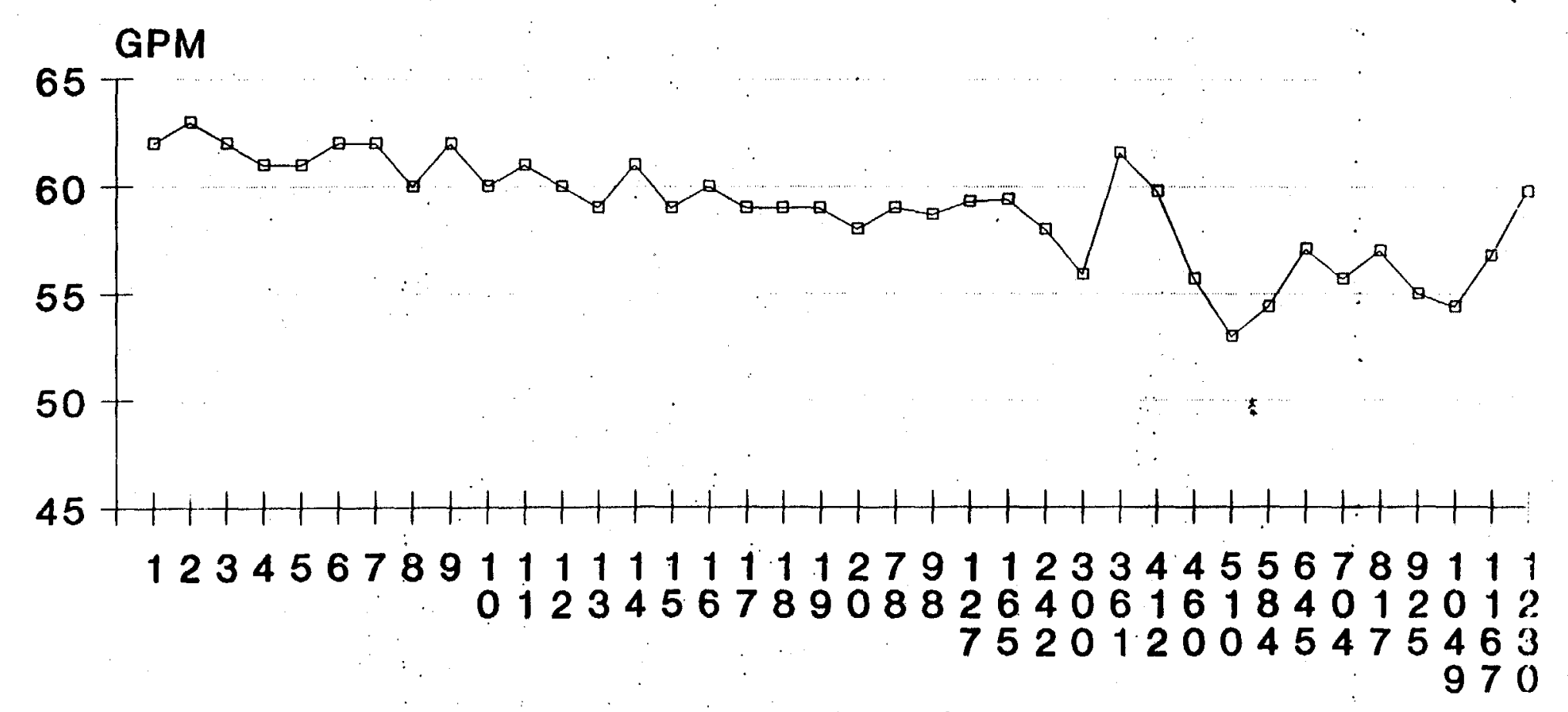

Time (min.)

$\rightarrow$ Pumping Rate

May 8 - 9 
The Theis non-equilibrium solution produced consistent estimates of transmissivity and storativity and was applied to data from all of the wells. The Hantush Jacob leaky solution was used to analyze drawdown data from several of the observation wells. The only data that displayed obvious leakage (or recharge), was from HSB 69A. The data collected from the other wells tended to fit the Theis non-leaky type curve satisfactorily.

\section{LEAKAGE AT HSB 69A}

Drawdown data from observation well HSB 69A were plotted on $10 \mathrm{~g}-\mathrm{log}$ paper and a curve matching procedure was used, based on the Hantush Jacob leaky confined aquifer analytical method, to estimate transmissivity, storativity,.. and hydraulic conductivity of the aquifer and the leaky confining bed. HSB $69 \mathrm{~A}$ is relatively close to the pumping well, HSB 84A, and both wells are partially penetrating wells, i.e. they are not open to the entire saturated thickness of the aquifer. When a pumping test is conducted using a pumping well that is partially penetrating, it is possible for the drawdown in nearby observation wells to be affected by vertical flow (partial penetration). When plotted, data that is affected by partial penetration can be mistaken for recharge or leakage. A mathematical method is presented in walton, 1988, for determining whether or not to expect partial penetration impacts. 'The equation is:

$\begin{aligned} r>1.5 b \mathrm{P}_{\mathrm{h}} / \mathrm{P}_{\mathrm{v}}, \text { where } \mathrm{r} & =\text { radial distance of observation } \\ & \text { well from pumping well. } \\ \mathrm{b} & =\text { aquifer thickness } \\ \mathrm{P}_{\mathrm{h}} & =\text { horizontal hydraulic conductivity } \\ & \text { of the aquifer. } \\ \mathrm{P}_{\mathrm{v}}= & \text { vertical hydraulic conductivity } \\ & \text { of the aquifer. }\end{aligned}$

The radial distance from HSB $69 \mathrm{~A}$ to HSB $84 \mathrm{~A}$ is 122 feet. Using the above equation with the following parameters:

aquifer thickness $=55 \mathrm{ft}$. based on lithologic logs, $\mathrm{P}_{\mathrm{h}} / \mathrm{P}_{\mathrm{V}}=1$ (aquifer isotropy is a basic assumption),

it can be determined that HSB $69 \mathrm{~A}$ is outside of the influence of partial penetration. Even if $P_{h} / P_{v}=2$, the results indicate that partial penetration impacts are insignificant.

Having eliminated the possibility of partial penetration impacts and assuming that the boundary conditions observed in the data collected at HSB $69 \mathrm{~A}$ resulted from vertical leakage across the confining bed, the hydraulic conductivity of the confining bed can be mathematically estimated using the following equation: 


$$
\begin{aligned}
K^{\prime}=T\left(\bar{b}^{\prime}\right) / B^{2}, \text { where } K^{\prime}= & \text { hydraulic conductivity of the } \\
& \text { confining bed. } \\
T \text { } & \text { transmissivity of the aquifer. } \\
b^{\prime}= & \text { thickness of the confining bed. } \\
B^{\prime}= & \text { variable determined from the } \\
& \text { curve matching procedure } \\
& \text { described in Hantush Jacob, } 1955 .
\end{aligned}
$$

Using the above equation and the following parameters:

$T=1292 \mathrm{ft}^{2} /$ day, based on data collected during the tests, $b^{\prime}=6$ feet, based on lithologic logs,

$B=1627$, based on $r / B=.075$ from the curve matching with $r$ previously identified as 122 feet,

the hydraulic conductivity of the leaky confining bed was estimated to be $0.0029 \mathrm{ft} / \mathrm{day}$. This is one to three orders of magnitude higher than estimates based on undisturbed samples collected in the confining bed from other points in the area in which the pumping test was conducted ${ }^{4}$. These results suggest the presence of a localized area of increased leakance in the vicinity of HSB $69 \mathrm{~A}$.

\section{OTHER RESULTS}

Transmissivity estimates based on the drawdown at each observation well using the Theis non-equilibrium solution were all very similar (with the exception of HSB 69A) and ranged from $1644 \mathrm{ft}^{2} /$ day to $2253 \mathrm{ft}^{2} /$ day with an average of $2013 \mathrm{ft}^{2} /$ day for Test one and from $1812 \mathrm{ft}^{2} /$ day to 2562 $\mathrm{ft}^{2} /$ day with an average of $2269 \mathrm{ft}^{2} /$ day for Test Two.

storage coefficient estimates ranged from 0.00022 to 0.00031 for Test one with an average value of 0.00027 and from 0.00020 to 0.00029 with an average of 0.00025 for Test Two (excluding HSB 69A): The transmissivity estimate using data collected at HSB 69A and the Hantush Jacol leaky solution was $1281 \mathrm{ft}^{2} /$ day for Test one and $1247 \mathrm{ft}^{2} /$ day for Test Two. The storage coefficient estimate based on HSB 69A data was 0.00038 for Test one and 0.00027 for Test Two.

A review of the geology of the tested aquifer reveals that the aquifer thickness is somewhat variable, ranging from 48 feet at the pumping well, to 94 , feet at HSB $83 \mathrm{~A}$ (see Figure 6). A basic assumption of the analytical solutions is that the saturated thickness of the pumped aquifer is uniform. The hydraulic conductivity of the aquifer material can be simply derived mathematically by dividing the transmissivity by the aquifer thickness. Because the aquifer thickness is apparently not uniform, hydraulic conductivity was estimated $u: i:-1$ both an average thickness of 65 feet and using the var $:$ : "hicknesses at each well. The following short table $1 \mathrm{i}:{ }^{*}$ : he range and average 


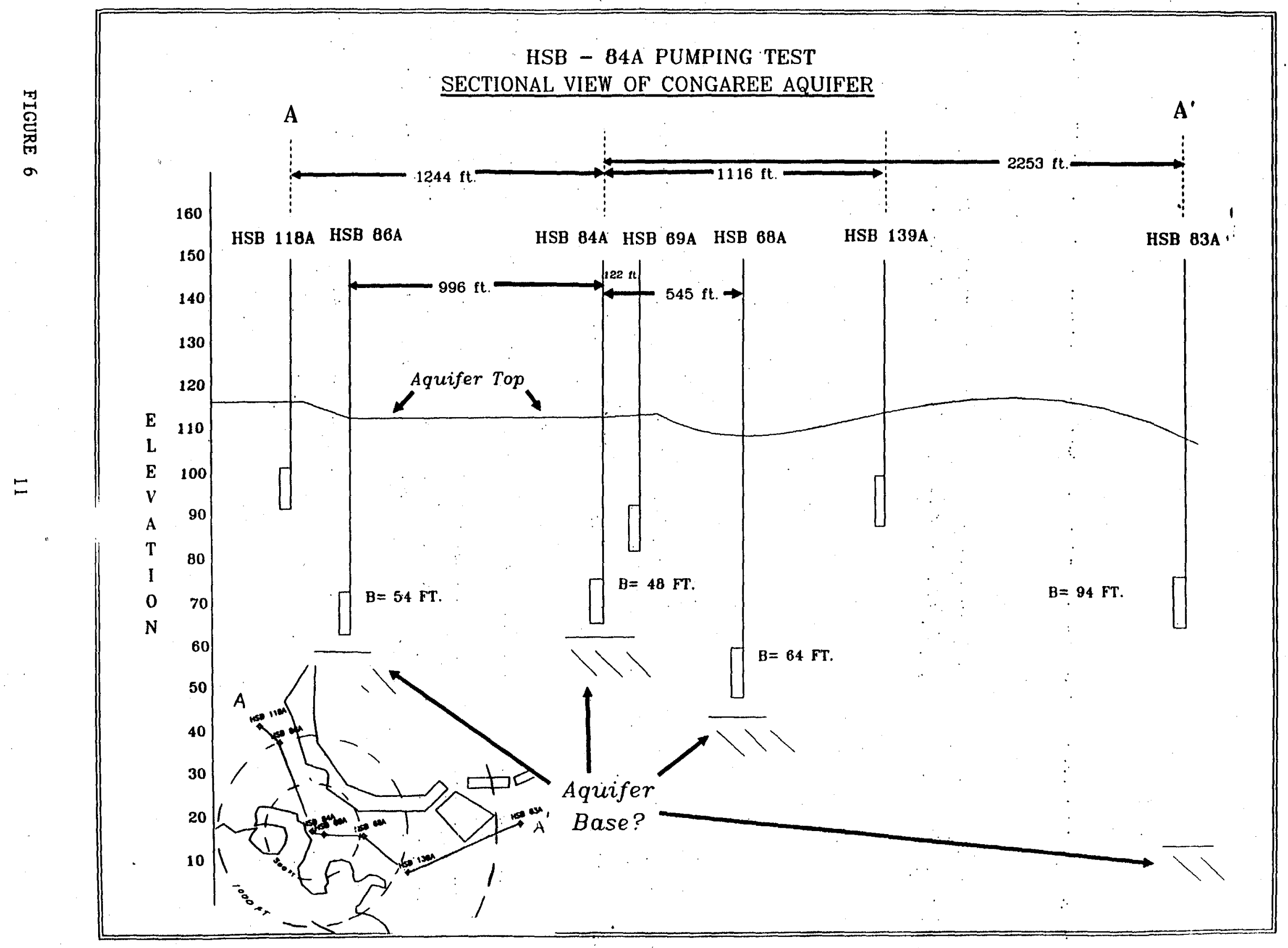


estimates of hydraulic conductivity based on uniform thickness and varying aquifer thicknesses:

TEST ONE

TEST TWO

Aquifer Thickness

VARIABLE

Range of $k_{h}$

Average $\mathrm{K}_{\mathrm{h}}$.

24 to $40 \mathrm{ft} / \mathrm{day}$

$31 \mathrm{ft} / \mathrm{day}$

VARIABLE

Aquifer Thickness

65 feet

20 to $35 \mathrm{ft} / \mathrm{day}$

$29 \mathrm{ft} / \mathrm{day}$

TEST TWO
VARIABLE
26 to $47 \mathrm{ft} / \mathrm{day}$
$34 \mathrm{ft} / \mathrm{day}$
$65 \mathrm{feet}$
19 to $39 \mathrm{ft} / \mathrm{day}$
$32 \mathrm{ft} / \mathrm{day}$

No hydraulic parameters were estimated from the drawdown in the pumping well (HSB 84A), because well efficiency was so low (estimated to be $27 \%$ ), that the data are essentially unusable. Specific capacity of the pumping well (yield per unit of drawdown) was calculated to be 1.8 gallons per minute/foot of drawdown or $1.8 \mathrm{gpm} / \mathrm{ft}$.

Observation wells screened in the upper water bearing. zones, above the pumped aquifer, did not show any drawdown during either test that could be attributed to pumping. Data collected from those wells is included in Appendices $A$ and $c$.

\section{SAMPLING}

$500 \mathrm{ml}$ samples were collected from the pumping well approximately every two hours during. both tests. The samples were taken to an on site $1 \mathrm{ab}$ and analyzed for tritium. The results were plotted versus time on graph paper and are included as Figure 7. Test Two was originally started at 9:00 a.m. on May 8 and was terminated after nineteen minutes because of problems with the generator, and then re-initiated three hours later. A sample was collected after one minute during the aborted test and the data is plotted with the rest of the Test Two data.

During Test Two, continuous measurements of temperature, $\mathrm{pH}$, conductivity, dissolved oxygen and ORP were made throughout the test using an in line Hydrolab tm. These data were not included in this report but will be included in a later report. 


\section{Tritium Concentrations Test One and Two}

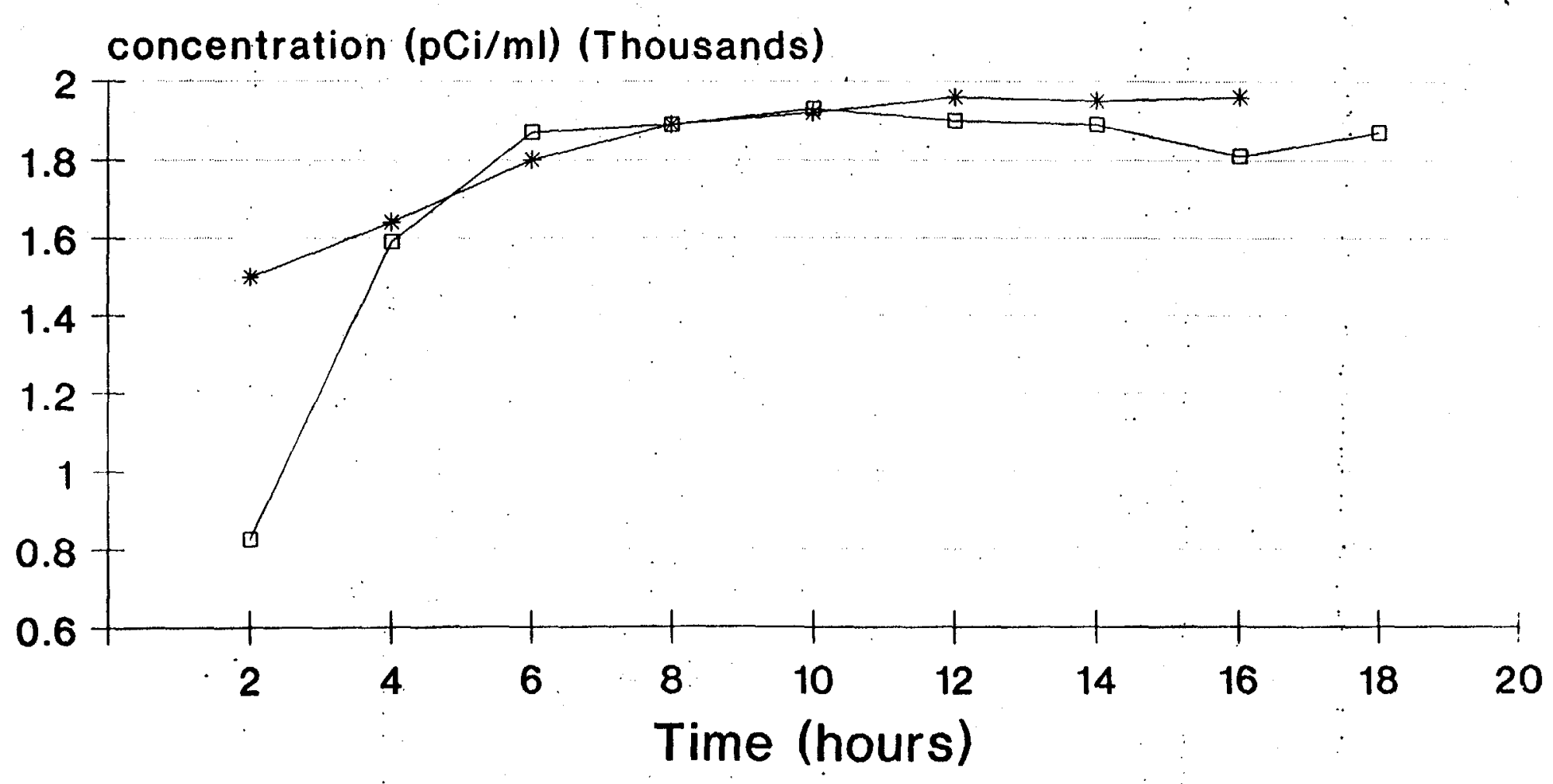

$$
\text { *-Test One } \rightarrow \text { - Test Two }
$$




\section{RECOMAENDATIONS FOR FUTURE WORK}

Pumping rate constancy was a problem in both tests, although test one was fairly uniform. The only power source that was available for this particular test was an older model generator. If a follow up test is attempted it would be adviseable to rent a new generator with a minimum of 72 hour's of fuel capacity.

The pumping well easily yielded 55 to 65 gallons per minute with fifteen to twenty feet of available drawdown remaining. Well efficiency was estimated at $27 \%$ using the distance drawdown analytical method and actual remaining available drawdown could have been as much as 40 feet. Based on that information, HSB $84 \mathrm{~A}$ could be expected to yield up to or greater than 100 gallons per minute. A fully penetrating 6" diameter well could believably yield 200 or more gallons per minute and such a pumping rate over several days might stress the system enough to draw some more definite conclusions about the contamination source and the characteristics of the confining bed. If a follow up test is attempted, a larger capacity pump should be used in HSB $84 \mathrm{~A}$, or a 6" diameter fully penetrating well should be installed nearby. There are plenty of Congaree wells in the area to use as observation wells and therefore no additional wells would need to be installed for that purpose.

The original plan for pumping HSB 84A, called for measuring water. levels during the drawdown and recovery portions of the test, in FSB 78A which is well outside of the influence of the pumping test. Because the data logger was available to collect precedent and antecedent water levels in HSB $84 \mathrm{~A}$ and HSB $69 \mathrm{~A}$ and because manpower resources were limited for the test, monitoring of FSB $78 \mathrm{~A}$ was not implemented. Sensitivity of the pressure transducers to changes in water level seemed to have some limitations i.e. there may have been some instrument drift when collecting the trend water levels which compounded the difficulty of computing barometric efficiency. Monitoring a well that is completely removed from the radius of influence of the pumping test is a very sound method for verifying the existence (or abserice) of external influences. Any future test should utilize that method.

\section{CONCLUSIONS}

Data collected and analyzed resulting from the pumping test do not clearly answer the question of why there is tritium contamination in the congaree aquifer at HSB 84A, however some leakage through the confining bed (green clay) was observed in the test results. The hydraulic conductivity of the confining bed was estimated to be 0.003 
feet/day-in the vicinity of observation well HSB 69A. This is one to three orders of magnitude higher than estimates made on undisturbed samples of the same clay layer collected at other wells in the area. The chemical data collected during the test do indicate that the pumpage was pulling in a constant concentration of tritium, with a rapid increase in the very early time of pumping and a quick levelling off to a.concentration approximately. ten times the pre-test level.

The wells screened in the zones above the pumped zone, did not display drawdown during either test. That information would tend to eliminate the possibility of any large opening, direct line type of communication existing between the Congaree and the upper zones in the immediate vicinity of HSB $84 \mathrm{~A}$.

Leakage (or recharge) was observed at HSB 69A. From the measured leakage, the hydraulic conductivity of the confining layer was estimated. A longer term test with a higher pumping rate and more observation wells might yield more information about leakage and lead to some stronger conclusions about the permeability and characteristics of the confining bed.

Although several analytical solutions were used to reduce the drawdown data, the Theis non-equilibrium solution was chosen as the most applicable for this particular exercise. Estimates of transmissivity based on the Theis solution at the different observation wells (excluding HSB $69 \mathrm{~A}$ ) ranged from $1644 \mathrm{ft}^{2} /$ day to $2253 \mathrm{ft}^{2} /$ day with an average of $2013 \mathrm{ft}^{2} / \mathrm{day}$ for Test one and from $1812 \mathrm{ft}^{2} / \mathrm{day}$ to $2562 \mathrm{ft}^{2} /$ day with an average of $2269 \mathrm{ft}^{2} /$ day for Test Two. Storage coefficient estimates ranged from 0.00022 to 0.00031 with an average of 0.00027 for Test one and from 0.00020 to 0.00029 with an average of 0.00025 for Test Two. The transmissivity estimate at HSB $69 \mathrm{~A}$ was estimated to be $1281 \mathrm{ft}^{2} /$ day for Test one and $1247 \mathrm{ft}^{2} /$ day for Test. Two, based on the Hantush Jacob leaky confined aquifer solution. The storage coefficients estimated at HSB $69 \mathrm{~A}$ were 0.00038 for Test one and 0.00027 for Test Two. 


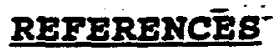

1. $\mathrm{CH}_{2} \mathrm{H}$ HILL, 1989. Congaree Aquifer Test, Savannah River site. Prepared for Westinghouse Savannah River Co. April 1989.

2. Heath, R.C., 1983. Basic Ground Water Hydrology, USGS Water Supply Paper 2220, 70pp:

3. G.M. Duffield and J.O. Rumbaugh III. AQTESOLV TM, Aquifer Test Solver, Version 1.00: October 1989.

4. Application For A Hazardous Waste Part B Permit, Savannah River Plant, United states Department of Energy, Volume V, H-Area Hazardous Waste Management Facility; Part 2: Sections E.1 - E.8, Table E.5-7, April 15, 1988. 


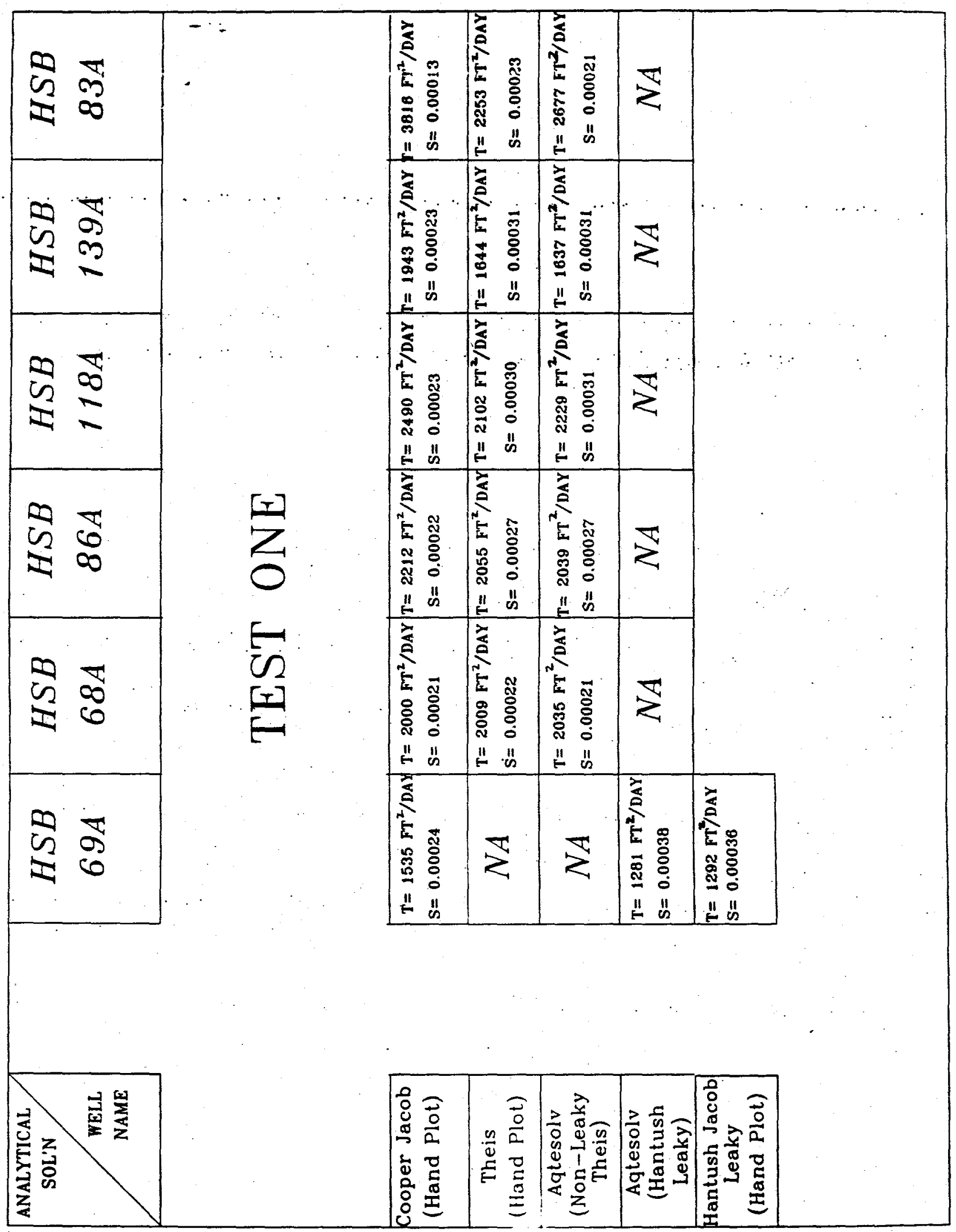




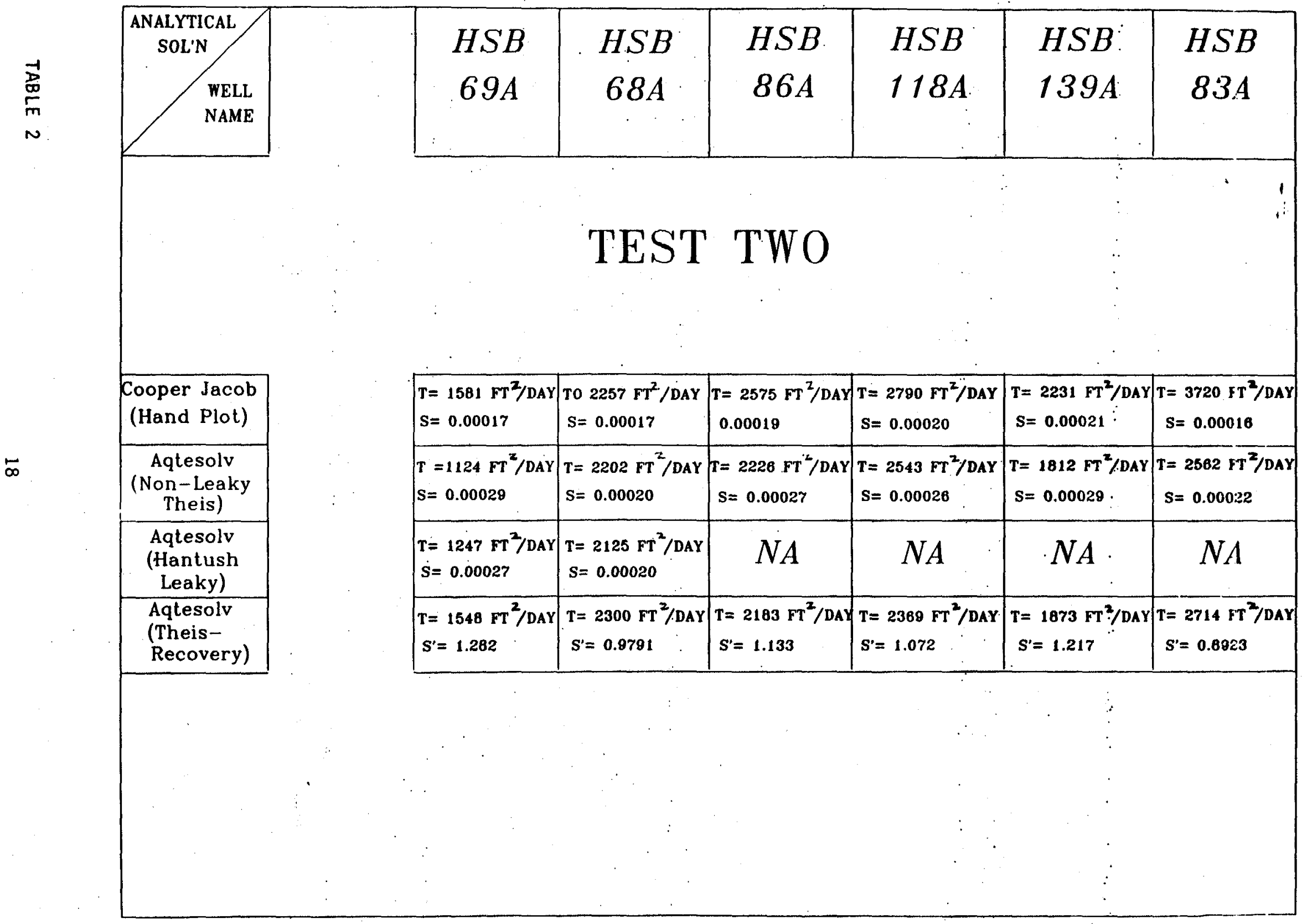


APPENDIX A

TEST ONE

DRATIDOWN DATA 
HSB 84A

APRIL 1, 1990 TEST ONE

DRAWDOWN DATA

ELAPSED TIME DRAWDOWN

\begin{tabular}{|c|c|c|}
\hline $\begin{array}{l}0.0000 \\
0.0083 \\
0.0166 \\
0.0250 \\
0.0333 \\
0.0416 \\
0.0500 \\
0.0583 \\
0.0666 \\
0.0750 \\
0.0833 \\
0.1000 \\
0.1166 \\
0.1333 \\
0.1500 \\
0.1666 \\
0.1833 \\
0.2000 \\
0.2166 \\
0.2333 \\
0.2500 \\
0.2666 \\
0.2833 \\
0.3000 \\
0.3166 \\
0.3333 \\
0.4166 \\
0.5000 \\
0.5833 \\
0.6666 \\
0.7500 \\
0.8333 \\
0.9166 \\
1.0000 \\
1.0833 \\
1.1666 \\
1.2500 \\
1.3333 \\
1.4166 \\
1.5000 \\
1.5833 \\
1.6666 \\
1.7500 \\
1.8333 \\
1.9166 \\
2.0000 \\
2.5000\end{array}$ & : & $\begin{array}{r}2.271 \\
3.454 \\
5.395 \\
5.836 \\
7.146 \\
7.761 \\
8.518 \\
8.692 \\
9.401 \\
9.607 \\
11.389 \\
12.777 \\
13.456 \\
15.002 \\
16.090 \\
15.617 \\
16.800 \\
19.403 \\
19.640 \\
20.933 \\
20.823 \\
21.895 \\
22.258 \\
21.801 \\
22.416 \\
23.205 \\
23.915 \\
25.429 \\
26.659 \\
25.445 \\
26.328 \\
27.480 \\
28.410 \\
26.817 \\
28.410 \\
29.215 \\
27.811 \\
28.300 \\
29.041 \\
28.505 \\
29.483 \\
29.436 \\
28.647 \\
28.868 \\
28.616 \\
28.647 \\
29.183\end{array}$ \\
\hline
\end{tabular}




\begin{tabular}{|c|c|}
\hline $\begin{array}{l}3.0000 \\
3.5000 \\
4.0000 \\
4.5000 \\
5.0000 \\
5.5000 \\
6.0000 \\
6.5000 \\
7.0000 \\
7.5000 \\
8.0000 \\
8.5000 \\
9.0000 \\
9.5000 \\
10.0000 \\
12.0000 \\
14.0000 \\
16.0000 \\
18.0000 \\
20.0000 \\
22.0000 \\
24.0000 \\
26.0000 \\
28.0000 \\
30.0000 \\
32.0000 \\
34.0000 \\
36.0000 \\
38.0000 \\
40.0000 \\
42.0000 \\
44.0000 \\
46.0000 \\
48.0000 \\
50.0000 \\
86.00000 \\
82.0000 \\
54.0000 \\
56.00000 \\
58.0000 \\
60.0000 \\
62.0000 \\
64.0000 \\
66.0000 \\
68.0000 \\
70.0000 \\
72.0000 \\
74.0000 \\
76.0000 \\
78.0000 \\
80.0000 \\
840000 \\
86000\end{array}$ & $\begin{array}{r}30.067 \\
30.083 \\
30.556 \\
30.177 \\
30.177 \\
30.445 \\
30.083 \\
30.288 \\
38.899 \\
30.177 \\
30.887 \\
30.414 \\
29.814 \\
29.704 \\
30.035 \\
30.020 \\
30.714 \\
30.193 \\
30.461 \\
30.793 \\
30.745 \\
30.666 \\
30.130 \\
30.714 \\
30.840 \\
31.581 \\
31.297 \\
31.723 \\
30.777 \\
32.165 \\
30.445 \\
30.840 \\
30.146 \\
31.155 \\
31.345 \\
31.187 \\
30.603 \\
30.998 \\
30.556 \\
31.534 \\
31.061 \\
31.455 \\
31.723 \\
31.424 \\
30.871 \\
31.171 \\
31.266 \\
31.455 \\
30.934 \\
31.969 \\
31.367 \\
32.417\end{array}$ \\
\hline
\end{tabular}




$\begin{array}{ll}90.0000 & 31.550 \\ 92.0000 & 31.360 \\ 94.0000 & 30.793 \\ 96.0000 & 31.187 \\ 98.0000 & 31.755 \\ 100.000 & 31.455 \\ 110.000 & 31.629 \\ 120.000 & 32.402 \\ 130.000 & 31.991 \\ 140.000 & 32.370 \\ 150.000 & 31.660 \\ 160.000 & 32.764 \\ 170.000 & 32.480 \\ 180.000 & 31.771 \\ 190.000 & 31.928 \\ 200.000 & 31.266 \\ 210.000 & 32.196 \\ 220.000 & 31.834 \\ 230.000 & 32.275 \\ 240.000 & 31.376 \\ 250.000 & 32.465 \\ 260.000 & 33.143 \\ 270.000 & 32.701 \\ 280.000 & 32.764 \\ 290.000 & 32.449 \\ 300.000 & 32.875 \\ 310.000 & 32.559 \\ 320.000 & 32.291 \\ 330.000 & 32.764 \\ 340.000 & 33.301 \\ 350.000 & 32.969 \\ 360.000 & 32.465 \\ 370.000 & 31.976 \\ 380.000 & 32.402 \\ 390.000 & 31.849 \\ 400.000 & 32.402 \\ 410.000 & 31.865 \\ 420.000 & 31.976 \\ 430.000 & 32.275 \\ 440.000 & 32.086 \\ 450.000 & 32.954 \\ 460.000 & 32.733 \\ 470.000 & 32.859 \\ 480.000 & 33.317 \\ 490.000 & 32.638 \\ 500.000 & 33.175 \\ 510.000 & 32.323 \\ 520.000 & 32.323 \\ 530.000 & 31.897 \\ 540.000 & 31.723 \\ 550.000 & 33.806 \\ 560.000 & 32.591 \\ 570.000 & 32.386 \\ 580.000 & \end{array}$




$\begin{array}{ll}590.000 & 32.118 \\ 600.000 & 31.660 \\ 610.000 & 32.449 \\ 620.000 & 32.433 \\ 630.000 & 33.080 \\ 640.000 & 32.496 \\ 650.000 & 32.859 \\ 660.000 & 32.070 \\ 670.000 & 31.360 \\ 680.000 & 31.865 \\ 690.000 & 31.502 \\ 700.000 & 32.260 \\ 710.000 & 32.622 \\ 720.000 & 32.402 \\ 730.000 & 31.692 \\ 740.000 & 32.544 \\ 750.000 & 32.591 \\ 760.000 & 32.512 \\ 770.000 & 31.692 \\ 780.000 & 31.897 \\ 790.000 & 31.644 \\ 800.000 & 32.417 \\ 810.000 & 33.916 \\ 820.000 & 31.897 \\ 830.000 & 32.323 \\ 840.000 & 32.291 \\ 850.000 & 32.827 \\ 860.000 & 33.096 \\ 870.000 & 33.175 \\ 880.000 & 32.307 \\ 890.000 & 32.323 \\ 900.000 & 32.528 \\ 910.000 & 33.395 \\ 920.000 & 32.701 \\ 930.000 & 32.780 \\ 940.000 & 32.102 \\ 950.000 & 33.317 \\ 960.000 & 30.635 \\ 970.000 & 33.253 \\ 980.000 & 33.632 \\ 990.000 & 33.159 \\ 1000.00 & 33.111 \\ 1100.00 & 32.275 \\ 1200.00 & 32.796 \\ 1300.00 & 4.322 \\ 1400.00 & 1.277\end{array}$


WELL NO.: $84 \mathrm{~A}$

PAGE 1 OF 2

DISTANCE FROM PUMPING WELL:

DATE: May 1, 1990

\begin{tabular}{|c|c|c|c|c|c|c|}
\hline TIME & $\begin{array}{c}\text { ELAPSED } \\
\text { TIME (MIN) }\end{array}$ & $\begin{array}{c}\text { DEPTH TO } \\
\text { WATER (TOC) }\end{array}$ & DRAWDOWN & $\begin{array}{c}\text { FLOW- } \\
\text { METER }\end{array}$ & Q (GPM) & MBAR \\
\hline $10: 15$ & 0 & 59.01 & 0 & 18149 & & 1013.9 \\
\hline $10: 21$ & 6 & 89.20 & 30.19 & 18445 & 49.3 & \\
\hline 10.28 & 13 & 89.63 & 30.62 & 18923 & 59.5 & \\
\hline 10.35 & 20 & 89.77 & 30.76 & 19338 & 59.3 & \\
\hline $10: 42$ & 27 & 89.98 & 30.97 & 19750 & 58.9 & \\
\hline $10: 50$ & 35 & 90.15 & 31.14 & 20225 & 59.4 & \\
\hline $11: 00$ & 45 & 90.25 & 31.24 & 208.15 & 59.0 & \\
\hline 11.35 & 80 & 90.60 & 31.59 & 22885 & 59.1 & 1014.25 \\
\hline $12: 35$ & 140 & 90.90 & 31.89 & 26448 & 59.4 & \\
\hline $13: 06$ & 171 & 91.07 & 32.06 & 28226 & 57.4 & 1013.88 \\
\hline $13: 55$ & 220 & 91.30 & 32.29 & 31287 & 62.5 & \\
\hline $14: 35$ & 260 & 91.65 & 32.64 & 33573 & 57.2 & 1013.2 \\
\hline $15: 10$ & 295 & 91.75 & 32.74 & 35680 & 60.2 & \\
\hline $16: 06$ & 351 & 91.72 & 32.71 & 39039 & 60.0 & \\
\hline $16: 55$ & 400 & 91.55 & 32.54 & 41964 & 59.7 & 1011.25 \\
\hline $17: 56$ & 461 & 91.55 & 32.54 & 45589 & $59: 4$ & 1011.54 \\
\hline $18: 55$ & 520 & 91.58 & 32.57 & 49170 & 60.7 & 1012.16 \\
\hline $20: 00$ & 585 & 91.61 & 32.60 & 52960 & 58.3 & 1012.38 \\
\hline $20: 52$ & 637 & 91.67 & 32.66 & 56070 & 59.8 & 1012.30 \\
\hline $22: 02$ & 707 & 91.67 & 32.66 & 60170 & 58.6 & 1013.55 \\
\hline $22: 56$ & 761 & 91.70 & 32.69 & 63368 & 59.2 & 1013.60 \\
\hline $00: 18$ & 843 & 91.60 & 2.59 & 68293 & 60.01 & 1013.9 \\
\hline & & & & & & \\
\hline
\end{tabular}




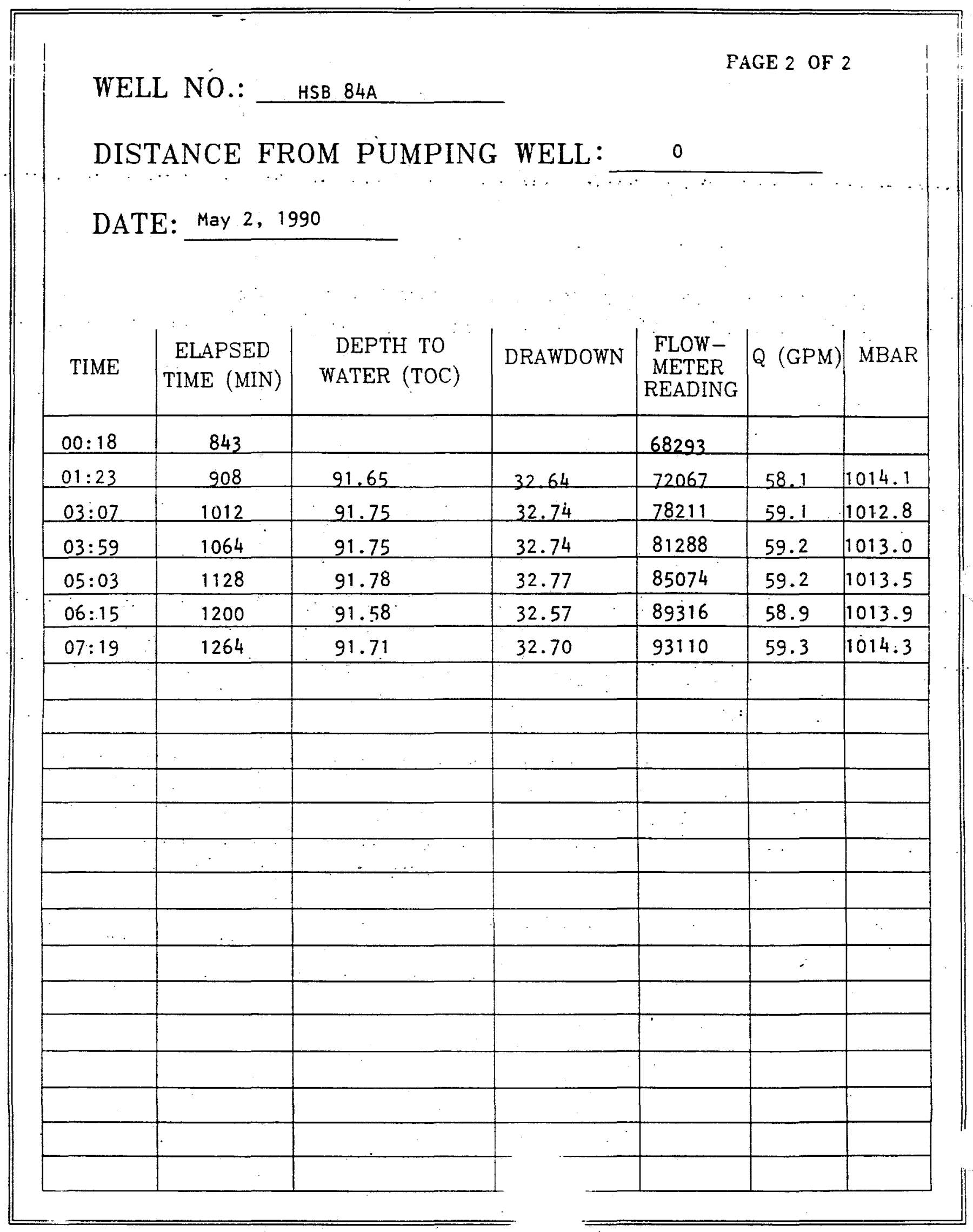




\section{HSB 84B \\ MAY 1,1990 \\ TEST ONE \\ DRAWDOWN DATA}

\section{ELAPSED TIME}

0.0000

0.0083

0.0166

0.0250

0.0333

0.0416

0.0500

0.0583

0.0666

0.0750

0.0833

0.1000

0.1166

0.1333

0.1500

0.1666

0.1833

0.2000

0.2166

0.2333

0.2500

0.2666

0.2833

0.3000

0.3166

0.3333

0.4166

0.5000

0.5833

0.6666

0.7500

0.8333

0.9166

1.0000

1.0833

1.1666

$1.2500^{\circ}$

1.3333

1.4166

1.5000

1.5833

1.6666

1.7500

1.8333

1.9166

2.0000

2.5000

3.0000

DRAWDOWN

$-0.015$

$-0.015$

$-0.015$

$-0.015$

$-0.015$

$-0.015$

$-0.015$

$-0.015$

$-0.015$

$-0.015$

$-0.015$

$-0.015$

$-0.015$

$-0.015$

$-0.015$

$-0.015$

$-0.015$

$-0.015$

$-0.015$

$-0.015$

$-0.015$

$-0.015$

$-0.015$

$-0.015$

$-0.015$

$-0.015$

$-0.015$

$-0.015$

$-0.015$

$-0.015$

$-0.031$

$-0.031$

$-0.031$

$-0.031$

$-0.031$

$-0.031$

$-0.031$

$-0.031$

$-0.031$

$-0.031$

$-0.015$

$-0.015$

$-0.015$

$-0.015$

$-0.015$

$-0.015$

$-0.015$

$-0.015$ 


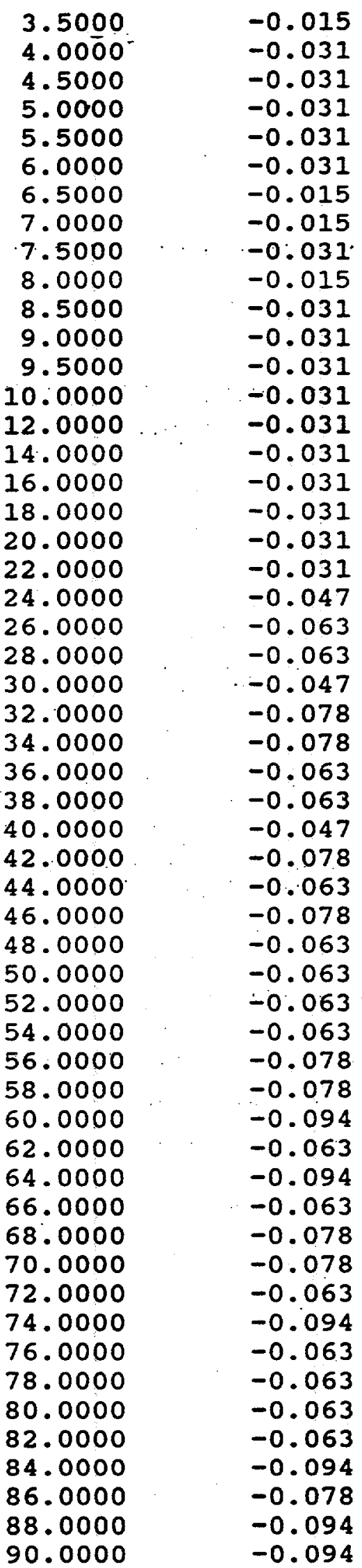




$\begin{array}{ll}92.0000 & -0.078 \\ 94.0000 & -0.078 \\ 96.0000 & -0.094 \\ 98.0000 & -0.063 \\ 100.000 & -0.078 \\ 110.000 & -0.063 \\ 120.000 & -0.078 \\ 130.000 & -0.078 \\ 140.000 & -0.078 \\ 150.000 & -0.078 \\ 160.000 & -0.063 \\ 170.000 & -0.110 \\ 180.000 & -0.094 \\ 190.000 & -0.078 \\ 200.000 & -0.063 \\ 210.000 & -0.094 \\ 220.000 & -0.063 \\ 230.000 & -0.078 \\ 240.000 & -0.078 \\ 250.000 & -0.078 \\ 260.000 & -0.078 \\ 270.000 & -0.078 \\ 280.000 & -0.078 \\ 290.000 & -0.078 \\ 300.000 & -0.078 \\ 310.000 & -0.047 \\ 320.000 & -0.063 \\ 330.000 & -0.031 \\ 340.000 & -0.047 \\ 350.000 & -0.063 \\ 360.000 & -0.063 \\ 370.000 & -0.063 \\ 380.000 & -0.126 \\ 390.000 & -0.063 \\ 400.000 & -0.078 \\ 410.000 & -0.126 \\ 420.000 & -0.063 \\ 430.000 & -0.078 \\ 440.000 & -0.047 \\ 450.000 & -0.047 \\ 460.000 & -0.063 \\ 470.000 & -0.063 \\ 480.000 & -0.063 \\ 490.000 & -0.063 \\ 500.000 & -0.063 \\ 510.000 & -0.047 \\ 520.000 & -0.047 \\ 530.000 & -0.047 \\ 540.000 & -0.063 \\ 550.000 & -0.063 \\ 560.000 & -0.063 \\ 570.000 & -0.063 \\ 580.000 & -0.063 \\ 590.000 & -0.063\end{array}$




$\begin{array}{ll}600.000 & -0.063 \\ 610.000 & -0.063 \\ 620.000 & -0.063 \\ 630.000 & -0.063 \\ 640.000 & -0.063 \\ 650.000 & -0.063 \\ 660.000 & -0.063 \\ 670.000 & -0.063 \\ 680.000 & -0.063 \\ 690.000 & -0.063 \\ 700.000 & -0.063 \\ 710.000 & -0.063 \\ 720.000 & -0.063 \\ 730.000 & -0.063 \\ 740.000 & -0.063 \\ 750.000 & -0.063 \\ 760.000 & -0.063 \\ 770.000 & -0.063 \\ 780.000 & -0.063 \\ 790.000 & -0.063 \\ 800.000 & -0.063 \\ 810.000 & -0.063 \\ 820.000 & -0.063 \\ 830.000 & -0.063 \\ 840.000 & -0.063 \\ 850.000 & -0.063 \\ 860.000 & -0.063 \\ 870.000 & -0.063 \\ 880.000 & -0.063 \\ 890.000 & -0.063 \\ 900.000 & -0.063 \\ 910.000 & -0.063 \\ 920.000 & -0.063 \\ 930.000 & -0.063 \\ 940.000 & -0.063 \\ 950.000 & -0.063 \\ 960.000 & -0.063 \\ 970.000 & -0.063 \\ 980.000 & -0.063 \\ 990.000 & -0.063 \\ 1000.00 & -0.063 \\ 1100.00 & -0.063 \\ 1200.00 & -0.047 \\ 1300.00 & -0.031 \\ 1400.00 & 0.110\end{array}$




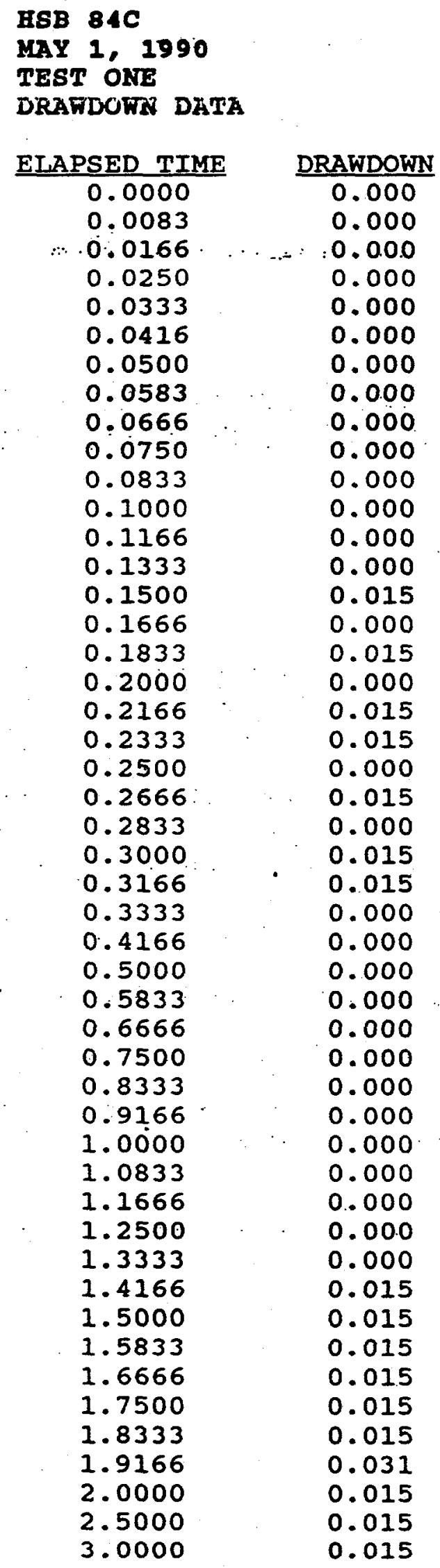




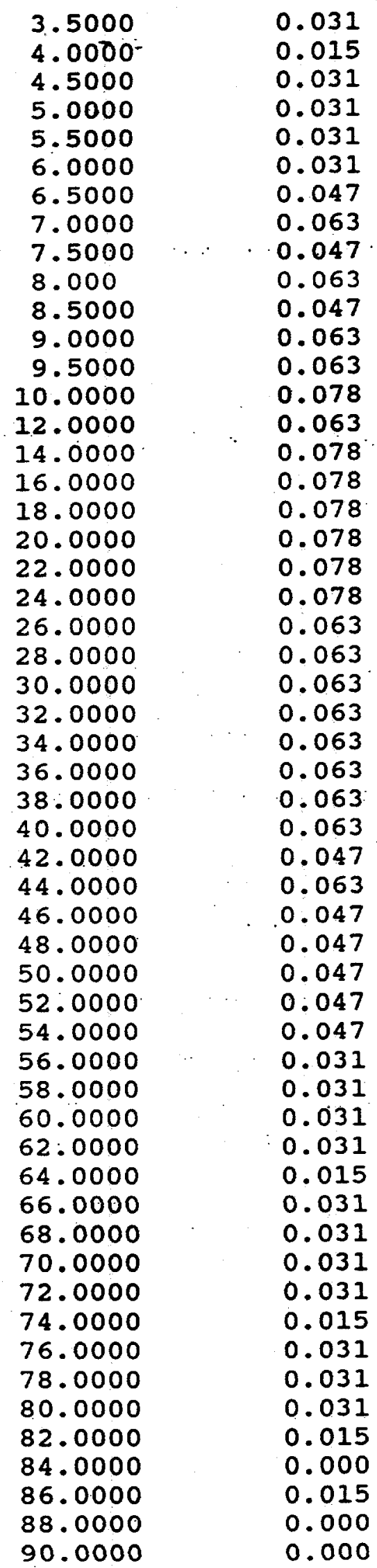




$\begin{array}{ll}92.0000 & 0.015 \\ 94.0000 & 0.000 \\ 96.0000 & 0.000 \\ 98.0000 & 0.015 \\ 100.000 & 0.000 \\ 110.000 & -0.015 \\ 120.000 & -0.047 \\ 130.000 & -0.063 \\ 140.000 & -0.078 \\ 150.000 & -0.078 \\ 160.000 & -0.078 \\ 170.000 & -0.078 \\ 180.000 & -0.094 \\ 190.000 & -0.094 \\ 200.000 & -0.063 \\ 210.000 & -0.094 \\ 220.000 & -0.078 \\ 230.000 & -0.078 \\ 240.000 & -0.078 \\ 250.000 & -0.078 \\ 260.000 & -0.063 \\ 270.000 & -0.078 \\ 280.000 & -0.078 \\ 290.000 & -0.094 \\ 300.000 & -0.078 \\ 310.000 & -0.063 \\ 320.000 & -0.078 \\ 330.000 & -0.063 \\ 340.000 & -0.078 \\ 350.000 & -0.078 \\ 360.000 & -0.078 \\ 370.000 & -0.078 \\ 380.000 & -0.126 \\ 390.000 & -0.078 \\ 400.000 & -0.094 \\ 410.000 & -0.110 \\ 420.000 & -0.078 \\ 430.000 & -0.094 \\ 440.000 & -0.063 \\ 450.000 & -0.078 \\ 460.000 & -0.078 \\ 470.000 & -0.078 \\ 480.000 & -0.078 \\ 490.000 & -0.078 \\ 500.000 & -0.078 \\ 510.000 & -0.078 \\ 520.000 & -0.078 \\ 530.000 & -0.078 \\ 540.000 & -0.078 \\ 550.000 & -0.078 \\ 560.000 & -0.078 \\ 570.000 & -0.098 \\ 580.000 & \\ 590.000 & \\ & \end{array}$




$\begin{array}{ll}600.000 & -0.094 \\ 610.000 & -0.094 \\ 620.000 & -0.094 \\ 630.000 & -0.094 \\ 640.000 & -0.094 \\ 650.000 & -0.094 \\ 660.000 & -0.094 \\ 670.000 & -0.094 \\ 680.000 & -0.094 \\ 690.000 & -0.094 \\ 700.000 & -0.094 \\ 710.000 & -0.094 \\ 720.000 & -0.094 \\ 730.000 & -0.094 \\ 740.000 & -0.094 \\ 750.000 & -0.094 \\ 760.000 & -0.094 \\ 770.000 & -0.094 \\ 780.000 & -0.094 \\ 790.000 & -0.094 \\ 800.000 & -0.094 \\ 810.000 & -0.094 \\ 820.000 & -0.094 \\ 830.000 & -0.094 \\ 840.000 & -0.094 \\ 850.000 & -0.094 \\ 860.000 & -0.094 \\ 870.000 & -0.094 \\ 880.000 & -0.094 \\ 890.000 & -0.094 \\ 900.000 & -0.094 \\ 910.000 & -0.094 \\ 920.000 & -0.094 \\ 930.000 & -0.094 \\ 940.000 & -0.094 \\ 950.000 & -0.094 \\ 960.000 & -0.094 \\ 970.000 & -0.094 \\ 980.000 & -0.094 \\ 990.000 & -0.094 \\ 1000.00 & -0.094 \\ 1100.00 & -0.173 \\ 1200.00 & -0.157 \\ 1300.00 & -0.126 \\ 1400.00 & -0.047 \\ & \\ 7300 & \end{array}$


HSB 84D

MAY 1, 1990

TEST ONE

DRAWDOWN DALA

ELAPSED TIME

0.0000

0.0083

0.0 .166

0.0250

0.0333

0.0416

0.0500

0.0583

0.0666

0.0750

0.0833

0.1000

0.1166

0.1333

0.1500

0.1666

0.1833

0.2000

0.2166

0.2333

0.2500

0.2666

0.2833

0.3000

0.3166

0.3333

0.4166

0.5000

0.5833

0.6666

0.7500

0.8333

0.9166

1.0000

1.0833

1. 1666

1.2500

1.3333

1.4166

1.5000

1.5833

1.6666

1.7500

1.8333

1.9166

2.0000

2. 5000

3.0000

DRAWDOWN

$-0.015$

0.000

0.000

0.000

0.000

0.000

$-0.015$

$-0.015$

$-0.015$

$-0.015$

$-0.015$

$-0.015$

$-0.015$

$-0.015$

$-0.015$

$-0.015$

0.000

$-0.015$

$-0.015$

$-0.015$

$-0.015$

$-0.015$

$-0.015$

$-0.015$

$-0.015$

$-0.015$

$-0.015$

$-0.015$

$-0.031$

$-0.031$

$-0.031$

$-0.031$

$-0.031$

$-0.031$

$-0.031$

$-0.031$

$-0.031$

$-0.031$

$-0.031$

$-0.031$

$-0.031$

$-0.031$

$-0.015$

$-0.031$

$-0.015$

$-0.015$

$-0.015$

$-0.015$ 


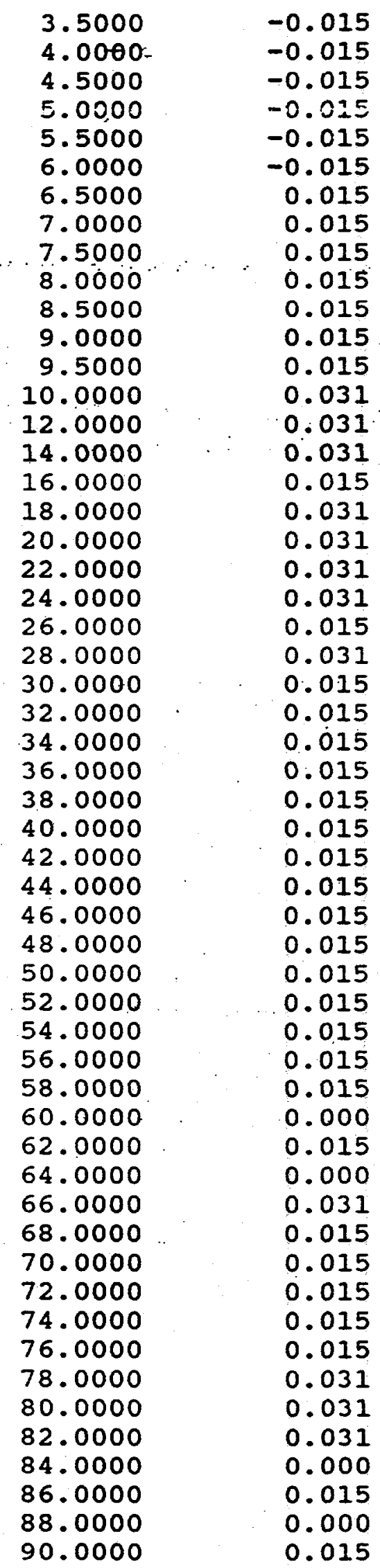




\begin{tabular}{|c|c|c|}
\hline $\begin{array}{l}94.0000 \\
96.0000 \\
100.000 \\
110.000 \\
120.000 \\
130.000 \\
140.000 \\
150.000 \\
160.000 \\
170.000 \\
180.000 \\
190.000 \\
200.000 \\
210.000 \\
220.000 \\
230.000 \\
240.000 \\
250.000 \\
260.000 \\
270.000 \\
280.000 \\
290.000 \\
300.000 \\
310.000 \\
320.000 \\
330.000 \\
340.000 \\
350.000 \\
360.000 \\
370.000 \\
380.000 \\
390.000 \\
400.000 \\
410.000 \\
420.000 \\
430.000 \\
440.000 \\
450.000 \\
460.000 \\
470.000 \\
480.000 \\
490.000 \\
500.000 \\
510.000 \\
520.000 \\
530.000 \\
540.000 \\
550.000 \\
570.000 \\
590.000\end{array}$ & $\cdot$ & $\begin{array}{l}0.031 \\
0.015 \\
0.015 \\
0.031 \\
0.015 \\
0.031 \\
0.015 \\
0.031 \\
0.015 \\
0.015 \\
0.031 \\
0.015 \\
0.015 \\
0.031 \\
0.031 \\
0.015 \\
0.031 \\
0.031 \\
0.031 \\
0.031 \\
0.015 \\
0.015 \\
0.031 \\
0.015 \\
0.031 \\
0.047 \\
0.031 \\
0.047 \\
0.031 \\
0.031 \\
0.01 \\
0.031 \\
0.015 \\
0.015 \\
0.015 \\
0.031 \\
0.015 \\
0.031 \\
0.015 \\
0.000 \\
0.031 \\
0.031 \\
0.015 \\
0.031 \\
0.031 \\
0.031 \\
0.031 \\
0.031 \\
0.031 \\
0.031 \\
0.031 \\
0.031 \\
0.031 \\
0\end{array}$ \\
\hline
\end{tabular}




\begin{tabular}{lr}
600.000 & 0.015 \\
610.000 & 0.015 \\
620.000 & 0.000 \\
630.000 & 0.000 \\
640.000 & 0.000 \\
650.000 & 0.000 \\
660.000 & 0.000 \\
670.000 & 0.000 \\
680.000 & 0.000 \\
690.000 & 0.000 \\
700.000 & 0.000 \\
710.000 & 0.000 \\
720.000 & 0.000 \\
730.000 & 0.000 \\
740.000 & 0.000 \\
750.000 & 0.000 \\
760.000 & 0.000 \\
770.000 & 0.000 \\
780.000 & 0.000 \\
790.000 & 0.000 \\
800.000 & -0.015 \\
810.000 & -0.015 \\
820.000 & -0.015 \\
830.000 & 0.000 \\
840.000 & -0.015 \\
850.000 & 0.000 \\
860.000 & 0.000 \\
870.000 & -0.015 \\
880.000 & -0.015 \\
890.000 & -0.015 \\
900.000 & -0.015 \\
910.000 & -0.015 \\
920.000 & -0.015 \\
930.000 & -0.015 \\
940.000 & -0.015 \\
950.000 & -0.015 \\
960.000 & -0.015 \\
970.000 & -0.031 \\
980.000 & -0.031 \\
990.000 & -0.031 \\
1000.00 & -0.031 \\
1100.00 & -0.031 \\
1200.00 & -0.031 \\
1300.00 & 0.000 \\
1400.00 & 0.031 \\
\hline $5 D$ &
\end{tabular}




\section{HSB 69 \\ MAY 1, 19900 \\ TEST ONE \\ DRAFDOWL DATA}

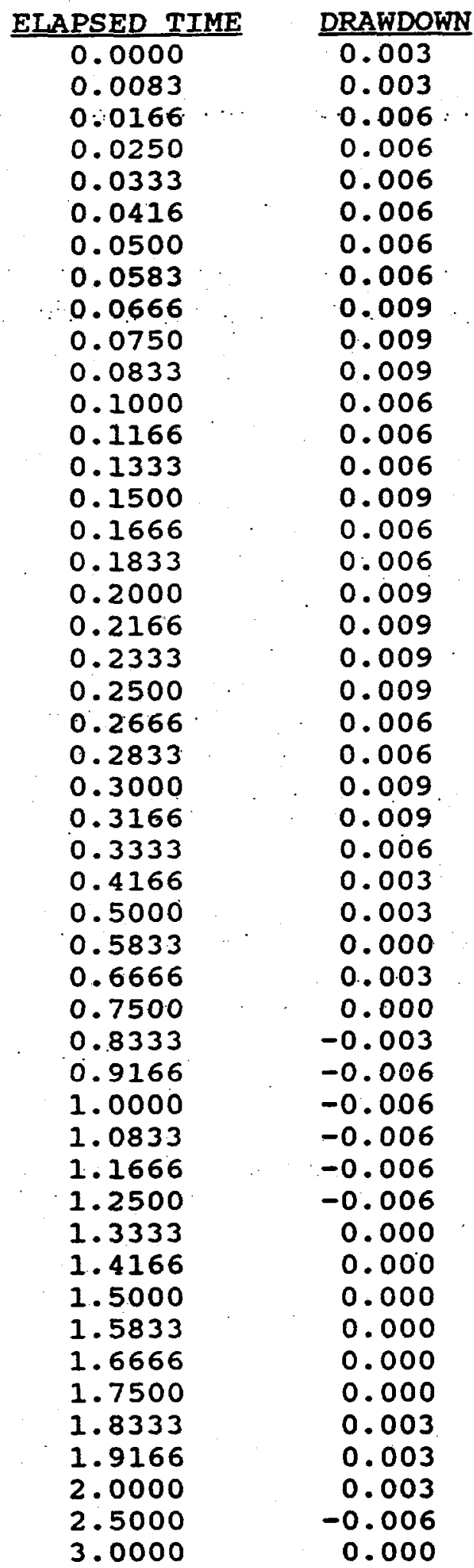




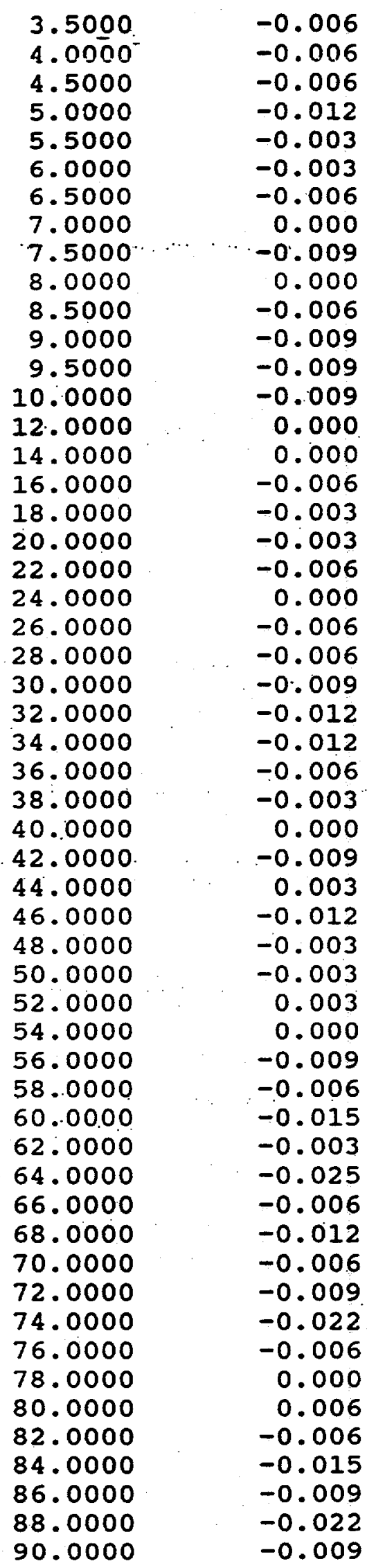




\begin{tabular}{lr}
92.0000 & -0.006 \\
94.0000 & -0.015 \\
96.0000 & -0.015 \\
98.0000 & 0.000 \\
100.000 & -0.003 \\
110.000 & -0.006 \\
120.000 & -0.009 \\
130.000 & -0.015 \\
140.000 & -0.012 \\
150.000 & -0.015 \\
160.000 & -0.012 \\
170.000 & -0.025 \\
180.000 & -0.018 \\
190.000 & -0.018 \\
200.000 & -0.009 \\
210.000 & -0.031 \\
220.000 & -0.006 \\
230.000 & -0.028 \\
240.000 & -0.028 \\
250.000 & -0.031 \\
260.000 & -0.012 \\
270.000 & -0.012 \\
280.000 & -0.018 \\
290.000 & -0.028 \\
300.000 & -0.015 \\
310.000 & -0.012 \\
320.000 & -0.018 \\
330.000 & -0.009 \\
340.000 & -0.018 \\
350.000 & -0.028 \\
360.000 & -0.025 \\
370.000 & -0.031 \\
380.000 & -0.056 \\
390.000 & -0.025 \\
400.000 & -0.031 \\
410.000 & -0.053 \\
420.000 & -0.025 \\
430.000 & -0.031 \\
440.000 & -0.022 \\
450.000 & -0.018 \\
460.000 & -0.025 \\
470.000 & -0.018 \\
480.000 & -0.022 \\
490.000 & -0.022 \\
500.000 & -0.022 \\
510.000 & -0.018 \\
520.000 & -0.022 \\
530.000 & -0.022 \\
540.000 & -0.025 \\
550.000 & -0.022 \\
570.0000 & -0.022 \\
590.000 & -0.025 \\
& -0.025 \\
\hline & -0.025
\end{tabular}




\begin{tabular}{ll}
600.000 & - \\
610.000 & -0.025 \\
620.000 & -0.025 \\
630.000 & -0.022 \\
640.000 & -0.022 \\
650.000 & -0.025 \\
660.000 & -0.025 \\
670.000 & -0.022 \\
680.000 & -0.022 \\
690.000 & -0.018 \\
700.000 & -0.018 \\
710.000 & -0.018 \\
720.000 & -0.018 \\
730.000 & -0.018 \\
740.000 & -0.018 \\
750.000 & -0.018 \\
760.000 & -0.018 \\
770.000 & -0.018 \\
780.000 & -0.018 \\
790.000 & -0.015 \\
800.000 & -0.018 \\
810.000 & -0.018 \\
820.000 & -0.018 \\
830.000 & -0.022 \\
840.000 & -0.018 \\
850.000 & -0.018 \\
860.000 & -0.018 \\
870.000 & -0.018 \\
880.000 & -0.022 \\
890.000 & -0.022 \\
900.000 & -0.022 \\
910.000 & -0.022 \\
920.000 & -0.022 \\
930.000 & -0.025 \\
940.000 & -0.025 \\
950.000 & -0.025 \\
960.000 & -0.028 \\
970.000 & -0.031 \\
980.000 & -0.031 \\
990.000 & -0.034 \\
1000.00 & -0.034 \\
1100.00 & -0.037 \\
1200.00 & -0.031 \\
1300.00 & -0.028 \\
1400.00 & -0.006 \\
\hline 10000 & 0.009
\end{tabular}

END 
HSB 69A

MAY 1,1990

TEST ONE

DRAWDOWI DATA

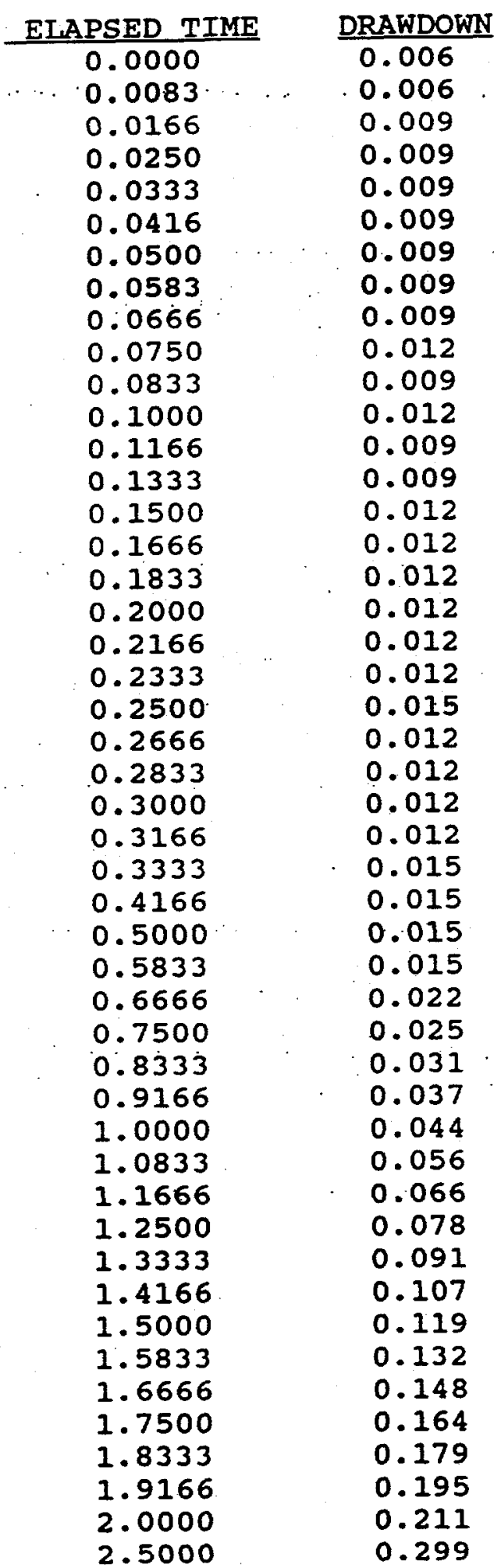




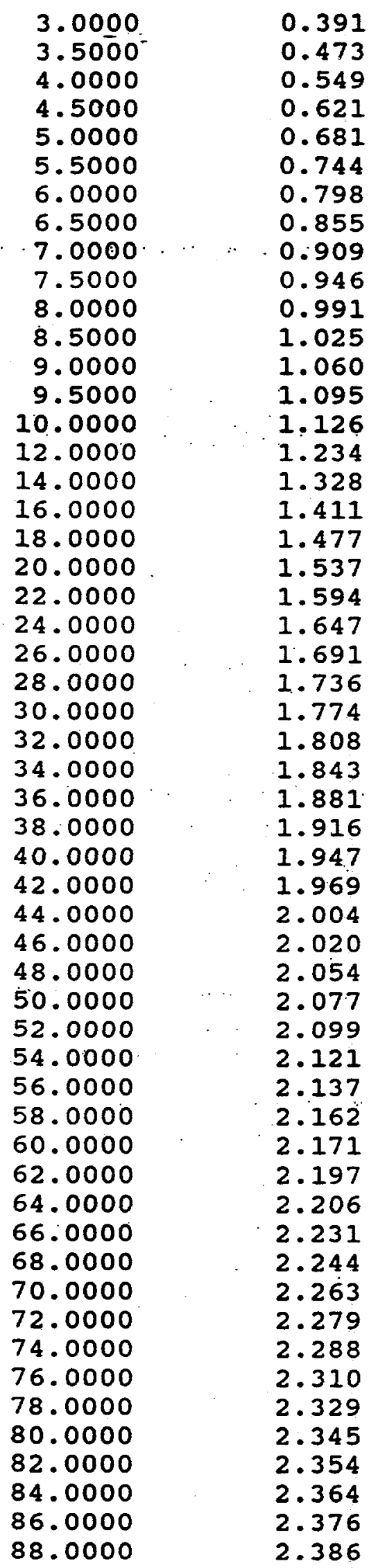




\begin{tabular}{|c|c|c|}
\hline 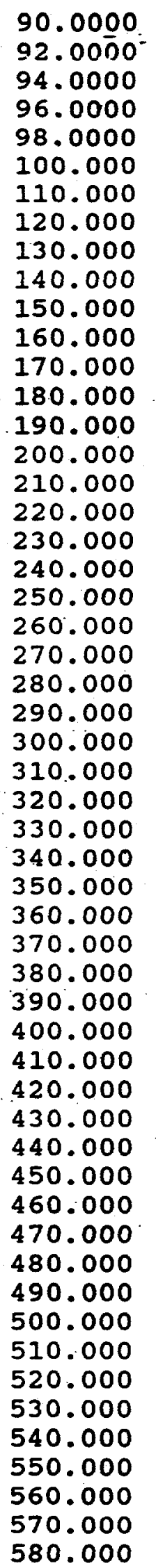 & $\cdot$ & $\begin{array}{l}2.402 \\
2.414 \\
2.424 \\
2.433 \\
2.455 \\
2.468 \\
2.500 \\
2.544 \\
2.582 \\
2.620 \\
2.651 \\
2.689 \\
2.717 \\
2.743 \\
2.777 \\
2.809 \\
2.822 \\
2.859 \\
2.869 \\
2.891 \\
2.916 \\
2.951 \\
2.973 \\
2.992 \\
3.001 \\
3.027 \\
3.046 \\
3.058 \\
3.077 \\
3.090 \\
3.093 \\
3.109 \\
3.118 \\
3.106 \\
3.140 \\
3.144 \\
3.134 \\
3.162 \\
3.172 \\
3.222 \\
3.232 \\
3.241 \\
3.251 \\
3.260 \\
3.267 \\
3.296 \\
3.301 \\
3.211\end{array}$ \\
\hline
\end{tabular}




\begin{tabular}{ll}
590.000 & 3.320 \\
600.000 & 3.327 \\
610.000 & 3.333 \\
620.000 & 3.342 \\
630.000 & 3.349 \\
640.000 & 3.355 \\
650.000 & 3.361 \\
660.000 & 3.368 \\
670.000 & 3.377 \\
680.000 & 3.383 \\
690.000 & 3.390 \\
700.000 & 3.396 \\
710.000 & 3.402 \\
720.000 & 3.412 \\
730.000 & 3.415 \\
740.000 & 3.424 \\
750.000 & 3.431 \\
760.000 & 3.437 \\
770.000 & 3.443 \\
780.000 & 3.450 \\
790.000 & 3.453 \\
800.000 & 3.456 \\
810.000 & 3.462 \\
820.000 & 3.466 \\
830.000 & 3.472 \\
840.000 & 3.475 \\
850.000 & 3.481 \\
860.000 & 3.488 \\
870.000 & 3.491 \\
880.000 & 3.497 \\
890.000 & 3.500 \\
900.000 & 3.507 \\
910.000 & 3.513 \\
920.000 & 3.519 \\
930.000 & 3.522 \\
940.000 & 3.525 \\
950.000 & 3.529 \\
960.000 & 3.532 \\
970.000 & 3.535 \\
980.000 & 3.541 \\
990.000 & 3.544 \\
1000.00 & 3.548 \\
1100.00 & 3.595 \\
1200.00 & 3.627 \\
1300.00 & 3.639 \\
1400.00 & 1.265 \\
\hline & \\
\hline &
\end{tabular}


WELL NO.: HSB 86A

PAGE 3 OP 3

DISTANCE FROM PUMPING WELL: $996 \mathrm{Ft}$

DATE: May 1,1990

\begin{tabular}{|c|c|c|c|c|c|c|}
\hline TIME & $\begin{array}{c}\text { ELAPSED } \\
\text { TIME (MIN) }\end{array}$ & $\begin{array}{c}\text { DEPTH TO } \\
\text { WATER (TOC) }\end{array}$ & DRAWDOWN & $\begin{array}{c}\text { FLOW- } \\
\text { METER }\end{array}$ & Q (GPM) & MBAR \\
\hline $10: 15$ & 0 & 95.56 & 0 & & & 1013.9 \\
\hline $11: 07$ & 52 & 95.67 & 0.11 & & & \\
\hline $11: 42$ & 87 & 95.78 & 0.22 & & & \\
\hline $12: 42$ & 147 & 95.92 & 0.36 & & & \\
\hline $13: 13$ & 178 & 96.01 & 0.45 & & & \\
\hline $14: 03$ & 228 & 96.08 & 0.52 & & & \\
\hline 14.45 & 270 & 96.14 & 0.58 & & & \\
\hline $15: 29$ & 314 & 96.20 & 0.64 & & & \\
\hline $16: 17$ & 362 & 96.25 & 0.69 & & & \\
\hline $17: 02$ & 407 & 96.30 & 0.74 & & & \\
\hline $18: 05$ & 470 & 96.36 & 0.80 & & & \\
\hline $19: 01$ & 526 & 96.40 & 0.84 & & & \\
\hline $20: 07$ & 592 & 96.45 & 0.89 & & & \\
\hline $20: 58$ & 643 & 96.50 & 0.94 & & & \\
\hline $22: 08$ & 713 & 96.50 & 0.94 & & & \\
\hline $23: 03$ & 768 & 96.57 & 1.01 & & & \\
\hline $00: 24$ & 849 & 96.60 & 1.04 & & & \\
\hline $01: 35$ & 920 & 96.64 & 1.08 & & & \\
\hline $03: 17$ & 1022 & 96.67 & 1.11 & & & \\
\hline $05: 13$ & 1138 & 96.72 & 1.16 & & & \\
\hline $07: 34$ & 1279 & 96.77 & 1.21 & & & \\
\hline & & & & & & \\
\hline
\end{tabular}




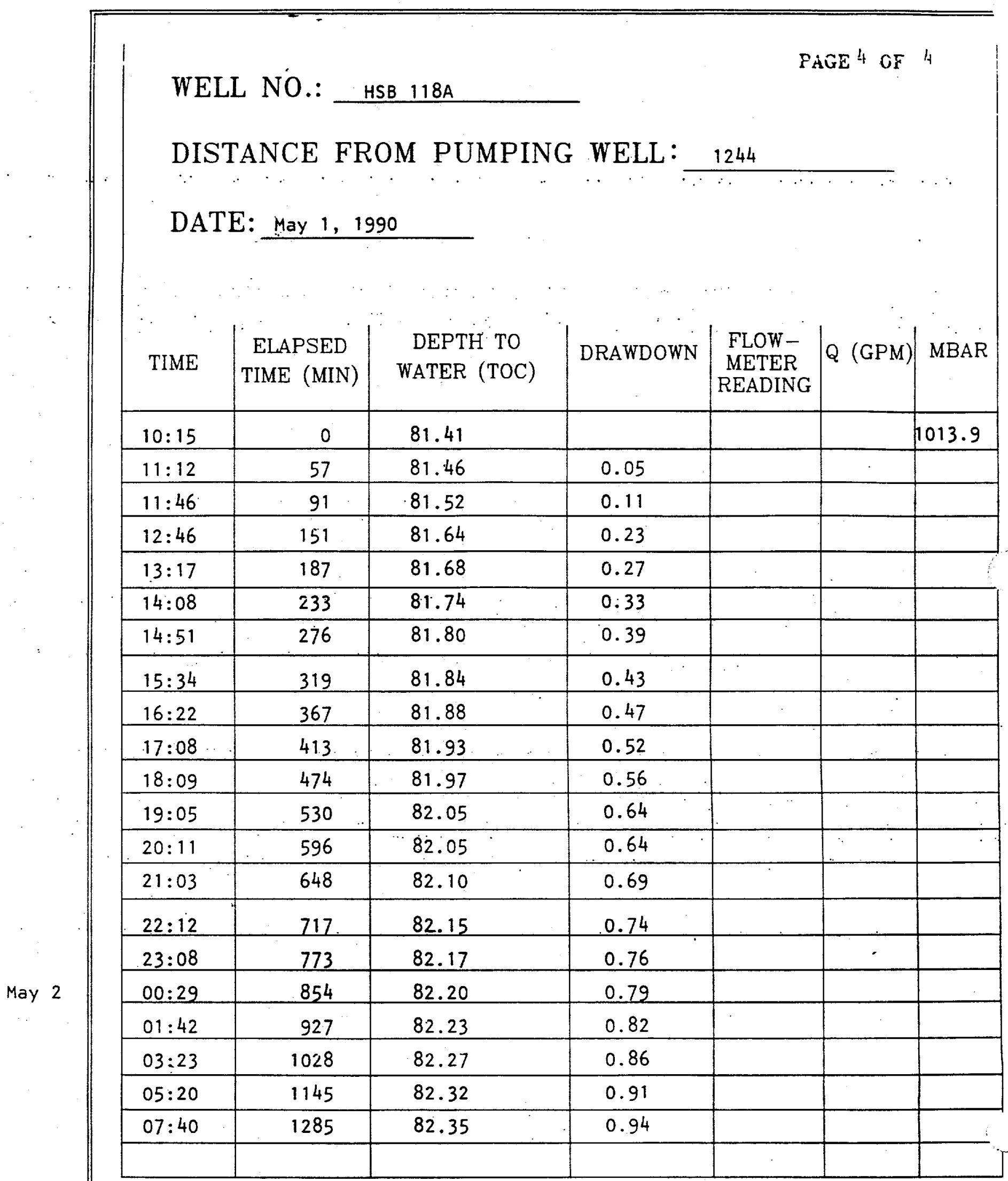


WELL NO.: HSB 68A

PAGE $_{5}$ OF 5

DISTANCE FROM PUMPING WELL: 545 FT

DATE: May 1,1990

\begin{tabular}{|c|c|c|c|c|c|c|}
\hline $\begin{array}{c}\text { TIME } \\
\text { (Min.) }\end{array}$ & $\begin{array}{c}\text { ELAPSED } \\
\text { TIME (MIN) }\end{array}$ & $\begin{array}{c}\text { DEPTH TO } \\
\text { WATER (TOC) }\end{array}$ & DRAWDOWN & $\begin{array}{c}\text { FLOW- } \\
\text { METER }\end{array}$ & Q (GPM) & MBAR \\
\hline $10: 15$ & 0 & 79.40 & 0 & & & 1013.9 \\
\hline $11: 18$ & 63 & 79.99 & 0.59 & & & \\
\hline 11.51 & 96 & 80.1 .5 & 0.75 & & & \\
\hline 12.51 & 156 & 80.35 & 0.95 & & & \\
\hline $13: 22$ & 187 & 80.40 & 1.00 & & & \\
\hline $14: 14$ & 239 & 80.51 & 1.11 & & & \\
\hline $14: 57$ & 282 & 80.58 & 1.18 & & & \\
\hline $15: 41$ & 326 & 80.65 & 1.25 & & & \\
\hline $16: 28$ & 373 & 80.71 & 1.31 & & & \\
\hline $17: 14$ & 419 & 80.75 & 1.35 & & & \\
\hline $18: 15$ & 480 & 80.82 & 1.42 & & & \\
\hline $19: 13$ & 538 & 80.87 & 1.47 & & & \\
\hline $20: 16$ & 601 & 80.95 & 1.55 & & & \\
\hline $21: 09$ & 654 & 80.99 & 1.59 & & & \\
\hline $22: 17$ & 722 & 81.04 & 1.64 & & & \\
\hline $23: 15$ & 780 & 81.10 & 1.71 & & & \\
\hline $00: 33$ & 858 & 81.10 & 1.71 & & & \\
\hline $01: 50$ & 935 & 81.13 & 1.73 & & & \\
\hline $03: 31$ & 1036 & 81.17 & 1.77 & & & \\
\hline $05: 27$ & 1152 & 81.22 & 1.82 & & & \\
\hline $07: 48$ & 1293 & 81.25 & 1.85 & & & \\
\hline & & & & & & \\
\hline & & & & & \\
\hline
\end{tabular}


WELL NO.: HSB 139A

PAGE 6 OF 6

DISTANCE FROM PUMPING WELL: $1116 \mathrm{FT}$

DATE: May 1, 1990

\begin{tabular}{|c|c|c|c|c|c|c|}
\hline TIME & $\begin{array}{c}\text { ELAPSED } \\
\text { TIME (MIN) }\end{array}$ & $\begin{array}{c}\text { DEPTH TO } \\
\text { WATER (TOC) }\end{array}$ & DRAWDOWN & $\begin{array}{c}\text { FLOW- } \\
\text { METRR } \\
\text { READING }\end{array}$ & Q (GPM) & MBAR \\
\hline $10: 15$ & 0 & 62.00 & 0 & & & 1013.9 \\
\hline $11: 22$ & 67 & 62.08 & 0.08 & & & \\
\hline 11.56 & 101 & 62.17 & 0.17 & & & \\
\hline 12.55 & 160 & 62.30 & 0.30 & & & \\
\hline $13: 26$ & 191 & 62.37 & 0.37 & & & \\
\hline $14: 19$ & 244 & 62.46 & 0.46 & & & \\
\hline $15: 02$ & 287 & 62.52 & 0.52 & & & \\
\hline $15: 46$ & 331 & 62.58 & 0.58 & & & \\
\hline $16: 33$ & 378 & 62.63 & 0.63 & & & \\
\hline $17: 19$ & 424 & 62.69 & 0.69 & & & \\
\hline $18: 20$ & 485 & 62.75 & 0.75 & & & \\
\hline $19: 19$ & 544 & 62.81 & 0.81 & & & \\
\hline $20: 20$ & 605 & 62.87 & 0.87 & & & \\
\hline $21: 15$ & 660 & 62.90 & 0.90 & & & \\
\hline 22.23 & 728 & 62.95 & 0.95 & & & \\
\hline $23: 20$ & 785 & 63.00 & 1.00 & & & \\
\hline $00: 38$ & 863 & 63.03 & 1.03 & & & \\
\hline $01: 56$ & 941 & 63.06 & 1.06 & & & \\
\hline $03: 38$ & 1043 & 63.11 & 1.11 & & & \\
\hline $05: 33$ & 1158 & 63.16 & 1.16 & & & \\
\hline $07: 55$ & 1300 & 63.20 & 1.20 & & & \\
\hline & & & & & & \\
\hline
\end{tabular}


WELL NO.: HSB 83A

PAGE 7 OF 7

DISTANCE FROM PUMPING WELL: $2253 \mathrm{FT}$

DATE: May 1, 1990

\begin{tabular}{|c|c|c|c|c|c|c|}
\hline TIME & $\begin{array}{c}\text { ELAPSED } \\
\text { TIME (MIN) }\end{array}$ & $\begin{array}{c}\text { DEPTH TO } \\
\text { WATER (TOC) }\end{array}$ & DRAWDOWN & $\begin{array}{c}\text { FLOW- } \\
\text { METER }\end{array}$ & Q (GPM) & MBAR \\
\hline $10: 15$ & 0 & 65.87 & 0 & & & 1013.9 \\
\hline $11: 28$ & 73 & 65.88 & 0.01 & & & \\
\hline $12: 02$ & 107 & 65.90 & 0.03 & & & \\
\hline 12.59 & 164 & 65.96 & 0.09 & & & \\
\hline $13: 32$ & 197 & 66.00 & 0.13 & & & \\
\hline $14: 26$ & 251 & 66.01 & 0.14 & & & \\
\hline $15: 52$ & 337 & 66.06 & 0.19 & & & \\
\hline $17: 26$ & 431 & 66.12 & 0.25 & & & \\
\hline $18: 26$ & 491 & 66.17 & 0.30 & & & \\
\hline $19: 25$ & 550 & 66.20 & 0.33 & & & \\
\hline $20: 27$ & 612 & 66.28 & 0.41 & & & \\
\hline $21: 21$ & 666 & 66.30 & 0.43 & & & \\
\hline $22: 29$ & 734 & 66.30 & 0.43 & & & \\
\hline $00: 43$ & 868 & 66.35 & 0.48 & & & \\
\hline $03: 46$ & 1051 & 66.40 & 0.53 & & & \\
\hline $05: 42$ & 1167 & 66.42 & 0.55 & & & \\
\hline $08: 05$ & 1310 & 66.45 & 0.58 & & & \\
\hline & & & & & & \\
\hline & & & & & & \\
\hline & & & & & & \\
\hline & & & & & & \\
\hline & & & & & & \\
\hline
\end{tabular}


$-\cdots$

APPENDIX B

TEST ONE

LEAKY AND MON-LEAKY BOLUTIONS

AQTESOLV ${ }^{\text {TL }}$ PLOTS 


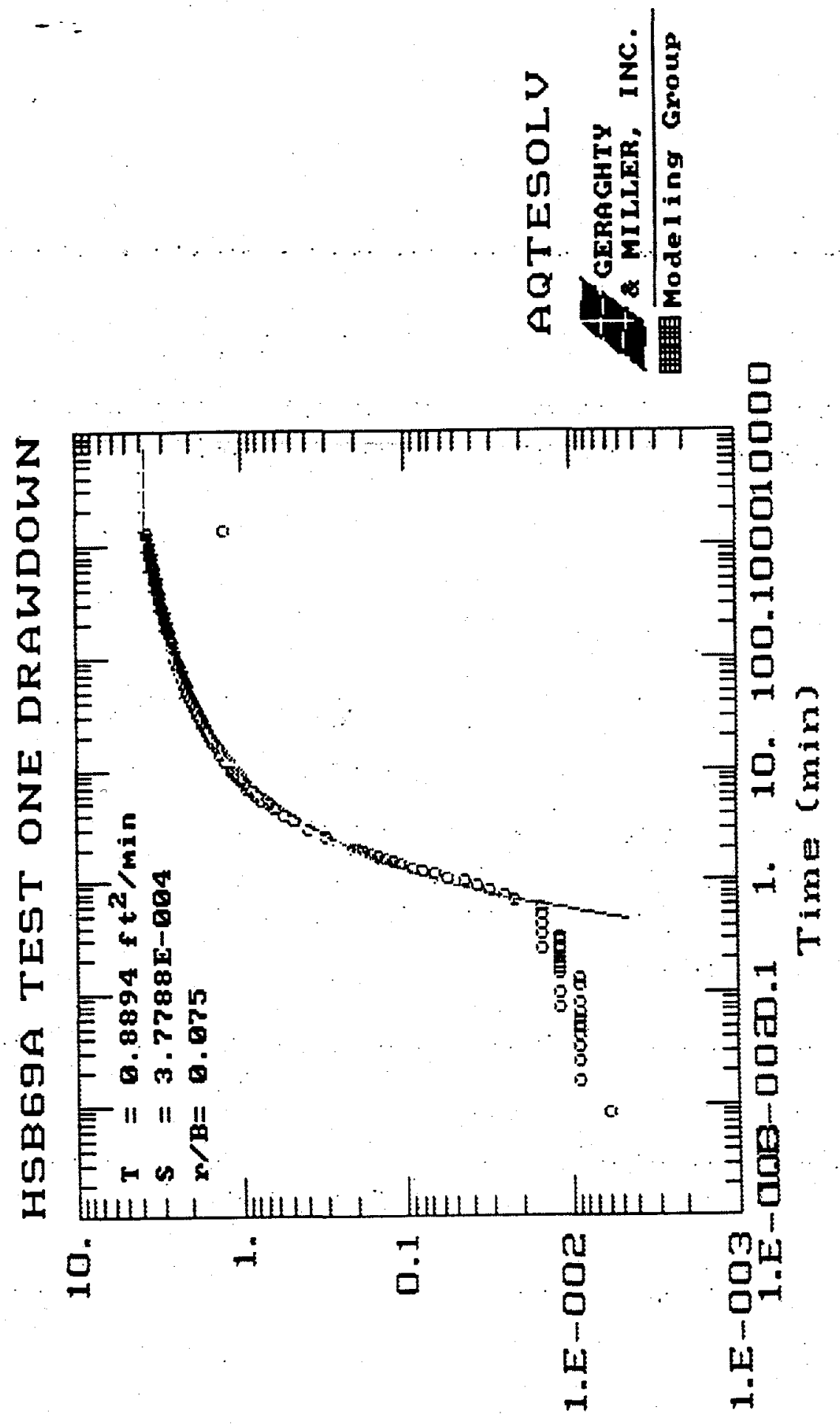

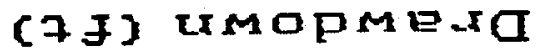




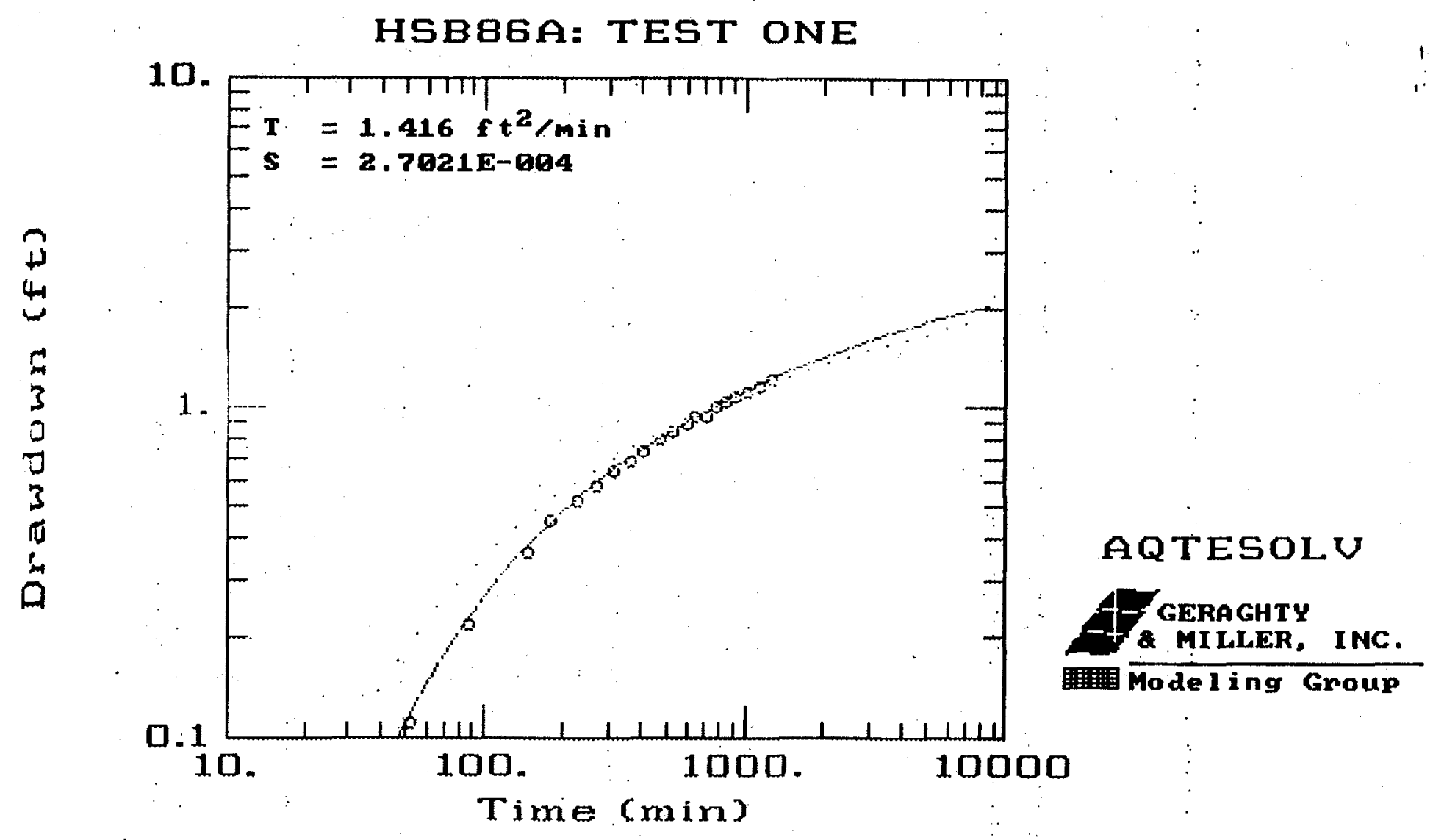




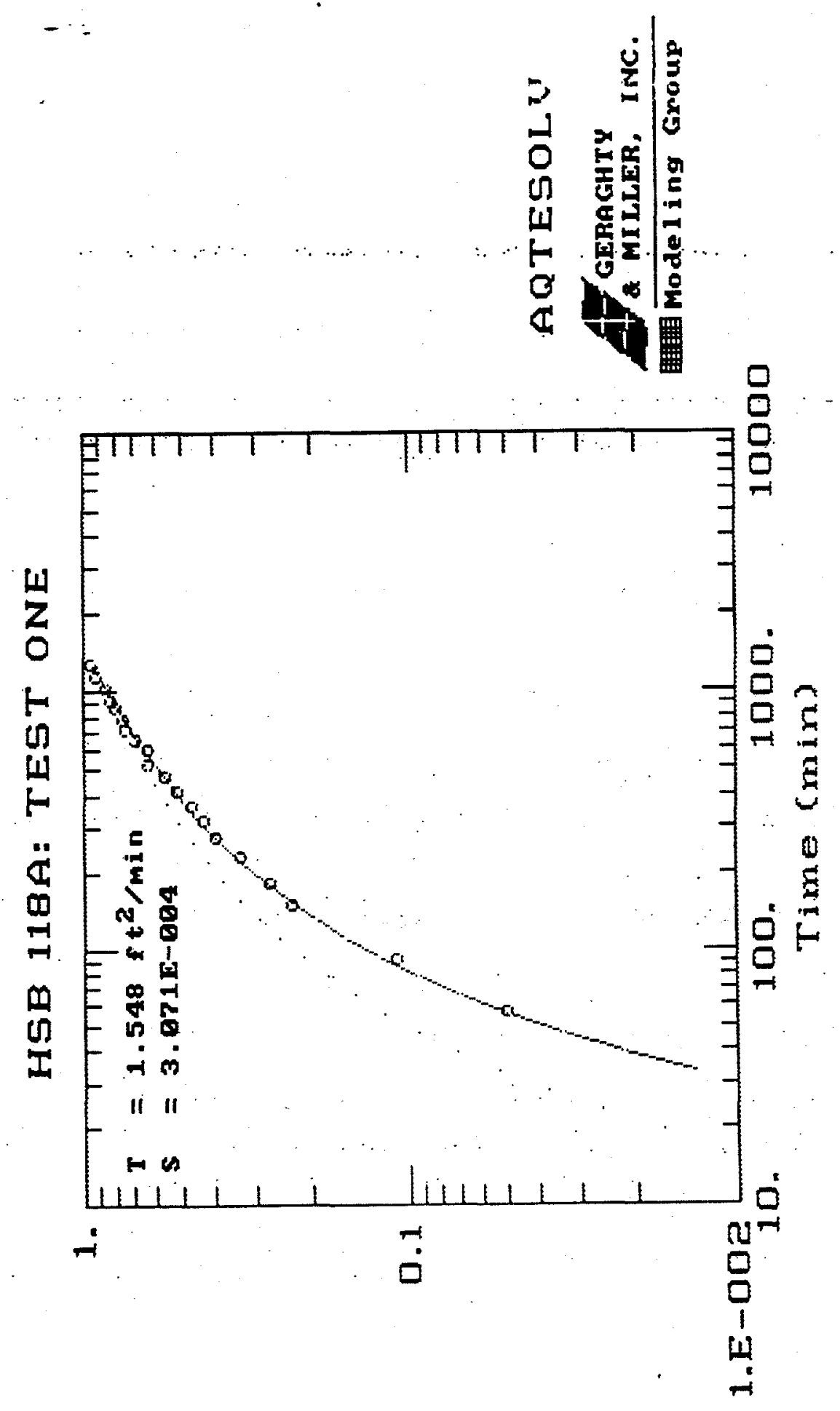

(7于) שMOPME- 


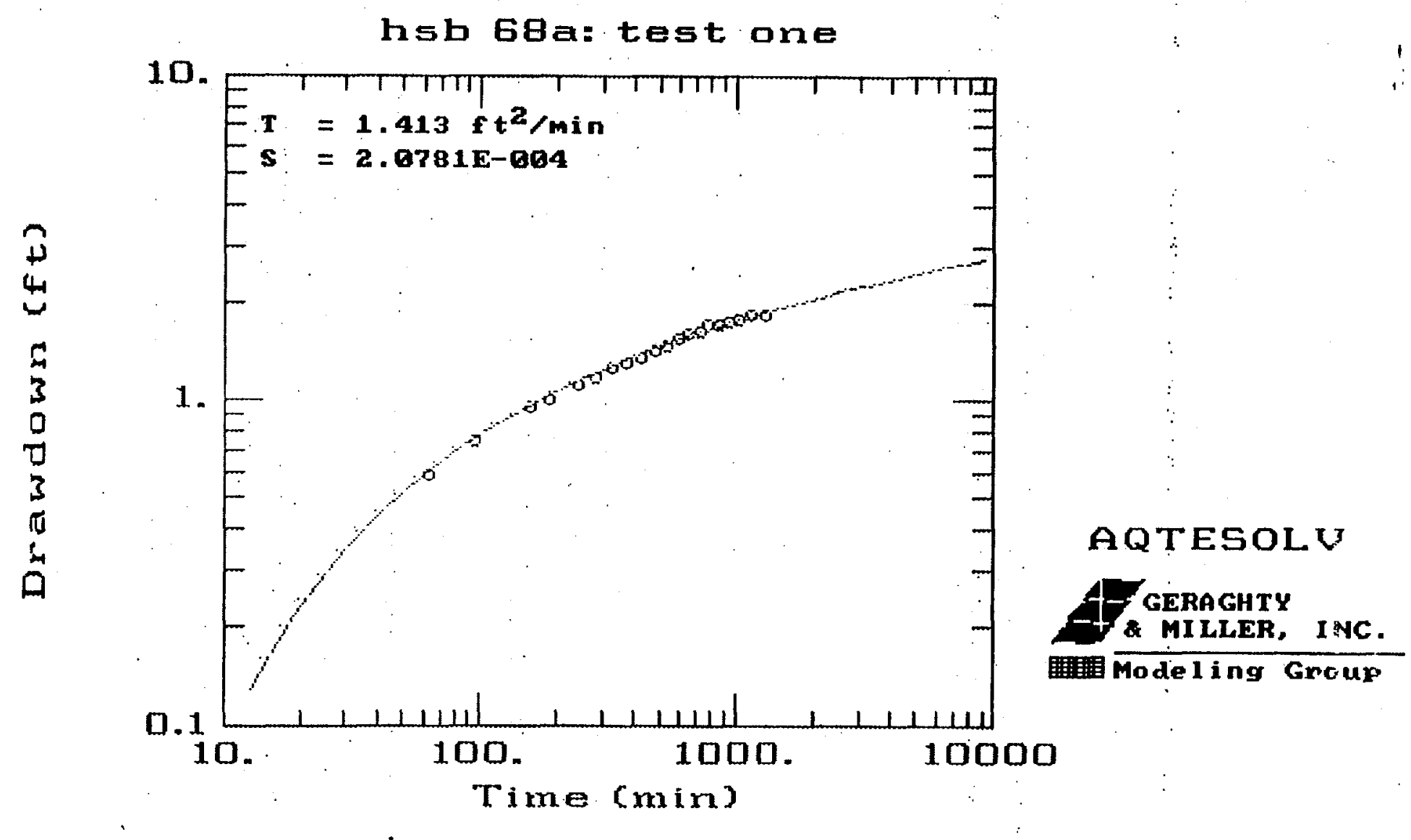




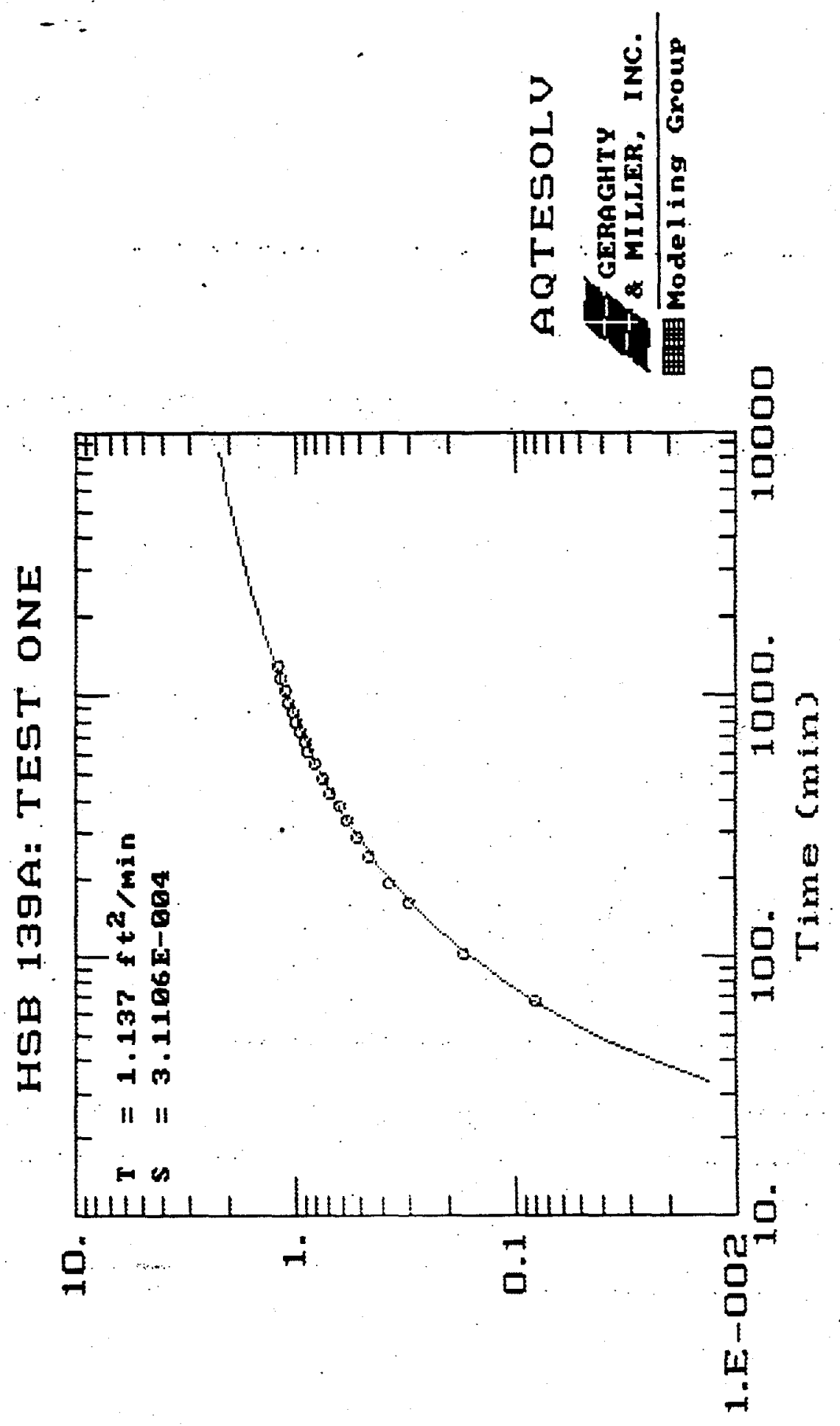

(7) यmnTmexa 


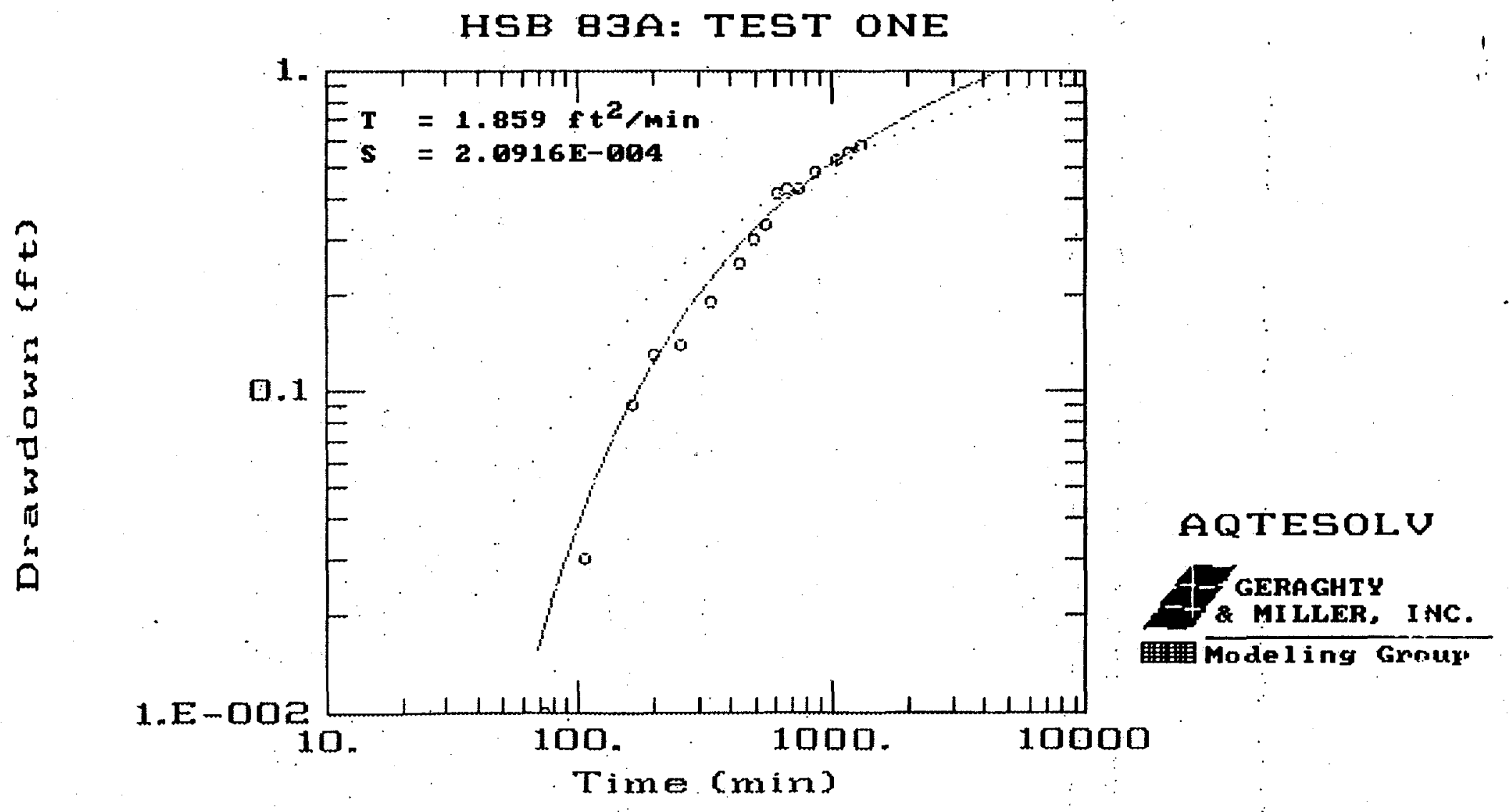


APPENDIX C

TEST TTO

DRANDOWN AND RECOVERY DATA 
WELL NO.: HSB 84A

PAGE 1 OF 3

DISTANCE FROM PUMPING WELL: 0

DATE: May 8, 1990

\begin{tabular}{|c|c|c|c|c|c|c|}
\hline TIME & $\begin{array}{c}\text { ELAPSED } \\
\text { TIME (MIN) }\end{array}$ & WETER (TOC) & DRAWDOWN & $\begin{array}{c}\text { FLOW- } \\
\text { METER } \\
\text { READING }\end{array}$ & Q (GPM) & MBAR \\
\hline $12: 30$ & 0 & 59.17 & 0 & 96790 & & \\
\hline $12: 31$ & 1 & & & 96852 & 62 & \\
\hline $12: 32$ & 2 & & & 96915 & 63 & \\
\hline $12: 33$ & 3 & & & 96977 & 62 & \\
\hline $12: 34$ & 4 & & & 97039 & 61 & \\
\hline $12: 35$ & 5 & & & 97100 & 61 & \\
\hline $12: 36$ & 6 & & 97162 & 62 & \\
\hline $12: 37$ & 7 & & 97224 & 62 & \\
\hline $12: 38$ & 8 & & & 97284 & 60 & \\
\hline $12: 39$ & 9 & & 97346 & 62 & \\
\hline $12: 40$ & 10 & & & 97406 & 60 & \\
\hline $12: 41$ & 11 & & 97467 & 61 & \\
\hline $12: 42$ & 12 & & & 97527 & 60 & \\
\hline $12: 43$ & 13 & & & 97586 & 59 & \\
\hline $12: 44$ & 14 & & & 97647 & 61 & \\
\hline $12: 45$ & 15 & & & 97706 & 59 & \\
\hline $12: 46$ & 16 & & & 97766 & 60 & \\
\hline $12: 47$ & 17 & & & 97825 & 59 & \\
\hline $12: 48$ & 18 & & & 97884 & 59 & \\
\hline $12: 49$ & 19 & & & 97943 & 59 & \\
\hline $12: 50$ & 20 & & & 98001 & 58 & \\
\hline $13: 48$ & 78 & 90.1 & $3 n .93$ & 101407 & 59 & 1020.7 \\
\hline
\end{tabular}


WELL NO.: HSB 84A

PAGE 2 OF 3

DISTANCE FROM PUMPING WELL: 0

DATE: May 8, 1990

\begin{tabular}{|c|c|c|c|c|c|c|}
\hline TIME & $\begin{array}{c}\text { ELAPSED } \\
\text { TIME (MIN) }\end{array}$ & $\begin{array}{c}\text { DEPTH TO } \\
\text { WATER (TOC) }\end{array}$ & DRAWDOWN & $\begin{array}{c}\text { FLOW- } \\
\text { METER } \\
\text { READING }\end{array}$ & (GPM) & MBAR \\
\hline $14: 08$ & 98 & $\sim 90.5$ & 31.33 & 102580 & 58.7 & 1020.45 \\
\hline $14: 37$ & 127 & $\sim 90.5$ & 31.33 & 104300 & 59.3 & \\
\hline $15: 15$ & 165 & $\sim 91.05$ & 31.88 & 106556 & 59.4 & 1020.0 \\
\hline $16: 32$ & 242 & $\sim 91.34$ & 32.17 & 111020 & 58.0 & 1018.9 \\
\hline $17: 30$ & 300 & $\sim 91.91$ & 32.74 & 114266 & 55.9 & 1018.8 \\
\hline $18: 31$ & 361 & $\sim 91.30$ & 32.13 & 118022 & 61.6 & 1018.24 \\
\hline $19: 22$ & 412 & $\sim 89.6$ & 30.43 & 121074 & 59.8 & 1018.3 \\
\hline $20: 10$ & 460 & NR & & 123749 & 55.7 & 018.15 \\
\hline $21: 00$ & 510 & $\sim 87.7$ & 28.53 & 126399 & 53.0 & 1018.35 \\
\hline $22: 14$ & 584 & $\sim 89.7$ & 30.53 & 130427 & 54.4 & 019.3 \\
\hline $23: 15$ & 645 & 89.92 & 30.75 & 133913 & 57.1 & 019.3 \\
\hline $00: 14$ & 704 & 89.25 & 30.08 & 137202 & 55.7 & 018.65 \\
\hline $02: 07$ & 817 & 89.82 & 30.69 & 143639 & 57.0 & 017.8 \\
\hline $03: 55$ & 925 & $\sim 89.0$ & 29.83 & 149580 & 59.0 & 016.7 \\
\hline $05: 59$ & 1049 & $\sim 89.9$ & 30.73 & 156.324 & 54.4 & 1017.4 \\
\hline $07: 57$ & 1167 & $\sim 92.17$ & 33.0 & 163025 & $56: 8$ & 1018.30 \\
\hline $09: 00$ & 1230 & $\sim 92.15$ & 32.98 & 166790 & 59.8 & 1018.37 \\
\hline & & Pump shut off at & $0: 00$ A. M. & & & \\
\hline & & RECGVER & & & & \\
\hline $10: 33$ & 33 & 60.97 & 1.8 & & & 1017.78 \\
\hline $10: 55$ & 55 & 60.70 & 1.53 & & & 1017.91 \\
\hline $11: 14$ & 74 & 60.50 & $\ddots 33$ & & & 1017.69 \\
\hline & & & & & & \\
\hline
\end{tabular}


WELL NO.: HSB 84A

PAGE 3 OF 3

\section{DISTANCE FROM PUMPING WELL: 0}

DATE: May 9, 1990

\begin{tabular}{|c|c|c|c|c|c|c|}
\hline TIME & $\begin{array}{l}\text { ELAPSED } \\
\text { TIME (MIN) }\end{array}$ & $\begin{array}{l}\text { DEPTH TO } \\
\text { WATER (TOC) }\end{array}$ & DRAWDOWN & \begin{tabular}{|c} 
FLOW- \\
METER \\
READING
\end{tabular} & $Q(\mathrm{GPM})$ & MBAR \\
\hline $11: 40$ & 100 & 60.37 & 1.2 & & & $1017 . \$$ \\
\hline $12: 53$ & 173 & 60.12 & 0.95 & & & 1016.96 \\
\hline $13: 14$ & 194 & 60.05 & 0.88 & & & 1016.80 \\
\hline $13: 36$ & 216 & 60.05 & 0.88 & & & 1016.54 \\
\hline $14: 29$ & 269 & 59.90 & 0.73 & & & 1015.83 \\
\hline $15: 30$ & 330. & 59.85 & 0.68 & & & 1014.18 \\
\hline $16: 30$ & 390 & 59.78 & 0.61 & & & 1013.45 \\
\hline $17: 32$ & 492 & 59.72 & 0.55 & & & 1012.29 \\
\hline $18: 33$ & -513 & -59.67 & 0.50 & & & 1012.45 \\
\hline $19: 27$ & 567 & 59.64 & 0.47 & & & 1012.0 \\
\hline $23: 49$ & 829 & -59.48 & 0.31 & & & 1008.8 \\
\hline $03: 59$ & 1079 & 59.40 & 0.23 & & & 1009.95 \\
\hline $07: 42$ & 1302 & 59.36 & 0.19 & & & 1006.1 \\
\hline $09: 47$ & 1427 & 59.33 & 0.16 & & & 1005.7 \\
\hline & & & & & & \\
\hline & & & & & & \\
\hline & & & & & & \\
\hline & & & & & & \\
\hline & & & & & & \\
\hline & & & & & & \\
\hline & & & & & & \\
\hline & & & & & & \\
\hline
\end{tabular}




\section{HSB 84A \\ MAY 8, 1990 \\ TEST TWO \\ DRAFDOWH DATA}

\section{ELAPSED TIME}

0.0000

0.0083

0.0166

0.0250

0.0333

0.0416

0.0500

0.0583

0.0666

0.0750

0.0833

0.1000

0.1166

0.1333

0.1500

0.1666

0.1833

0.2000

0.2166

0.2333

0.2500

0.2666

0.2833

0.3000

0.3166

0.3333

0.4166

0.5000

0.5833

0.6666

0.7500

0.8333

0.9166

1.00 .00

1.0833

1.1666

1.2500

1.3333

1.4166

1.5000

1.5833

1.6666

1.7500

1.8333

1.9166

2.0000

2.5000

3.0000

DRAWDOWN

8.423

0.914

4.511

4.275

5.600

6.262

7.414

8.408

8.565

9.906

10.458

11.626

13.156

14.497

15.380

16.374

17.415

19.371

18.267

20.649

19.529

21.043

22.242

21.075

23. 236

22.337

25.066

26.092

29.247

29.625

28.631

30.335

28.379

29.215

30.303

30.934

28.836

30.430

29.909

30.934

31.613

30.240

30.351

29.688

31.171

31.408

31.644

31.818 


\begin{tabular}{ll}
3.5000 & 30.382 \\
4.0000 & 31.266 \\
4.5000 & 30.808 \\
5.0000 & 31.376 \\
5.5000 & 31.424 \\
6.0000 & 30.950 \\
6.5000 & 30.903 \\
7.0000 & 31.644 \\
7.5000 & 32.512 \\
8.0000 & 32.323 \\
8.5000 & 31.865 \\
9.0000 & 31.960 \\
9.5000 & 30.856 \\
10.0000 & 31.218 \\
12.0000 & 30.098 \\
14.0000 & 29.499 \\
16.0000 & 31.392 \\
18.0000 & 30.982 \\
20.0000 & 30.682 \\
22.0000 & 30.982 \\
24.0000 & 30.130 \\
26.0000 & 31.944 \\
28.0000 & 31.991 \\
30.0000 & 30.193 \\
32.0000 & 32.291 \\
34.0000 & 31.392 \\
36.0000 & 31.203 \\
38.0000 & 32.307 \\
40.0000 & 30.698 \\
42.0000 & 31.234 \\
44.0000 & 32.433 \\
46.0000 & 30.083 \\
48.0000 & 29.956 \\
50.0000 & 30.272 \\
52.0000 & 31.250 \\
54.0000 & 31.629 \\
56.0000 & 31.502 \\
58.0000 & 31.597 \\
60.0000 & 30.272 \\
62.0000 & 30.919 \\
64.0000 & 30.635 \\
66.0000 & 31.502 \\
68.0000 & 31.613 \\
70.0000 & 30.603 \\
72.0000 & 30.083 \\
74.0000 & 30.745 \\
76.0000 & 32.433 \\
78.0000 & 31.786 \\
80.0000 & 29.941 \\
82.0000 & 30.998 \\
84.0000 & 31.439 \\
86.0000 & 32.165 \\
88.0000 & 31.234 \\
90.0000 & \\
\hline &
\end{tabular}




\begin{tabular}{ll}
92.0000 & 30.572 \\
94.0000 & 30.840 \\
96.0000 & 31.360 \\
98.0000 & 31.360 \\
100.000 & 30.840 \\
110.000 & 32.496 \\
120.000 & 31.234 \\
130.000 & 31.045 \\
140.000 & 31.771 \\
150.000 & 32.938 \\
160.000 & 30.303 \\
170.000 & 31.913 \\
180.000 & 31.676 \\
190.000 & 31.991 \\
200.000 & 31.471 \\
210.000 & 31.518 \\
220.000 & 31.865 \\
230.000 & 31.771 \\
240.000 & 33.080 \\
250.000 & 33.253 \\
260.000 & 32.338 \\
270.000 & 31.960 \\
280.000 & 32.922 \\
290.000 & 31.203 \\
300.000 & 33.001 \\
310.000 & 33.190 \\
320.000 & 33.159 \\
330.000 & 32.149 \\
340.000 & 33.206 \\
350.000 & 31.802 \\
360.000 & 32.338 \\
370.000 & 31.613 \\
380.000 & 32.544 \\
390.000 & 32.275 \\
400.000 & 31.045 \\
410.000 & 31.124 \\
420.000 & 30.319 \\
430.000 & 31.218 \\
440.000 & 29.909 \\
450.000 & 30.714 \\
460.000 & 29.530 \\
470.000 & 29.373 \\
480.000 & 27.716 \\
490.000 & 27.322 \\
500.000 & 28.174 \\
510.000 & 27.038 \\
520.000 & 29.530 \\
530.000 & 27.716 \\
540.000 & 28.000 \\
550.000 & 28.805 \\
560.000 & 29.657 \\
570.000 & 30.761 \\
580.000 & 30.556 \\
590.000 & 30.256 \\
& \\
\hline 150
\end{tabular}




\begin{tabular}{ll}
600.000 & 29.846 \\
610.000 & 31.439 \\
620.000 & 31.360 \\
630.000 & 30.761 \\
640.000 & 30.934 \\
650.000 & 30.777 \\
660.000 & 31.360 \\
670.000 & 31.250 \\
680.000 & 29.893 \\
690.000 & 29.199 \\
700.000 & 28.332 \\
710.000 & 29.830 \\
720.000 & 29.310 \\
730.000 & 33.127 \\
740.000 & 32.039 \\
750.000 & 32.985 \\
760.000 & 31.755 \\
770.000 & 30.729 \\
780.000 & 30.777 \\
790.000 & 30.240 \\
800.000 & 30.430 \\
810.000 & 29.925 \\
820.000 & 30.351 \\
830.000 & 31.203 \\
840.000 & 29.688 \\
850.000 & 29.783 \\
860.000 & 29.672 \\
870.000 & 30.461 \\
880.000 & 29.404 \\
890.000 & 30.477 \\
900.000 & 30.272 \\
910.000 & 29.105 \\
920.000 & 28.205 \\
930.000 & 29.120 \\
940.000 & 28.805 \\
950.000 & 29.294 \\
960.000 & 28.947 \\
970.000 & 28.773 \\
980.000 & 28.663 \\
990.000 & 28.963 \\
1000.00 & 29.389 \\
1100.00 & 31.550 \\
1200.00 & 32.654 \\
$E N D$ & \\
& \\
\hline 70
\end{tabular}




\section{HSB $84 B$ \\ MAY $8,1 \overline{9} 90$ \\ TEST TWO \\ DRAWDOWN DATA}

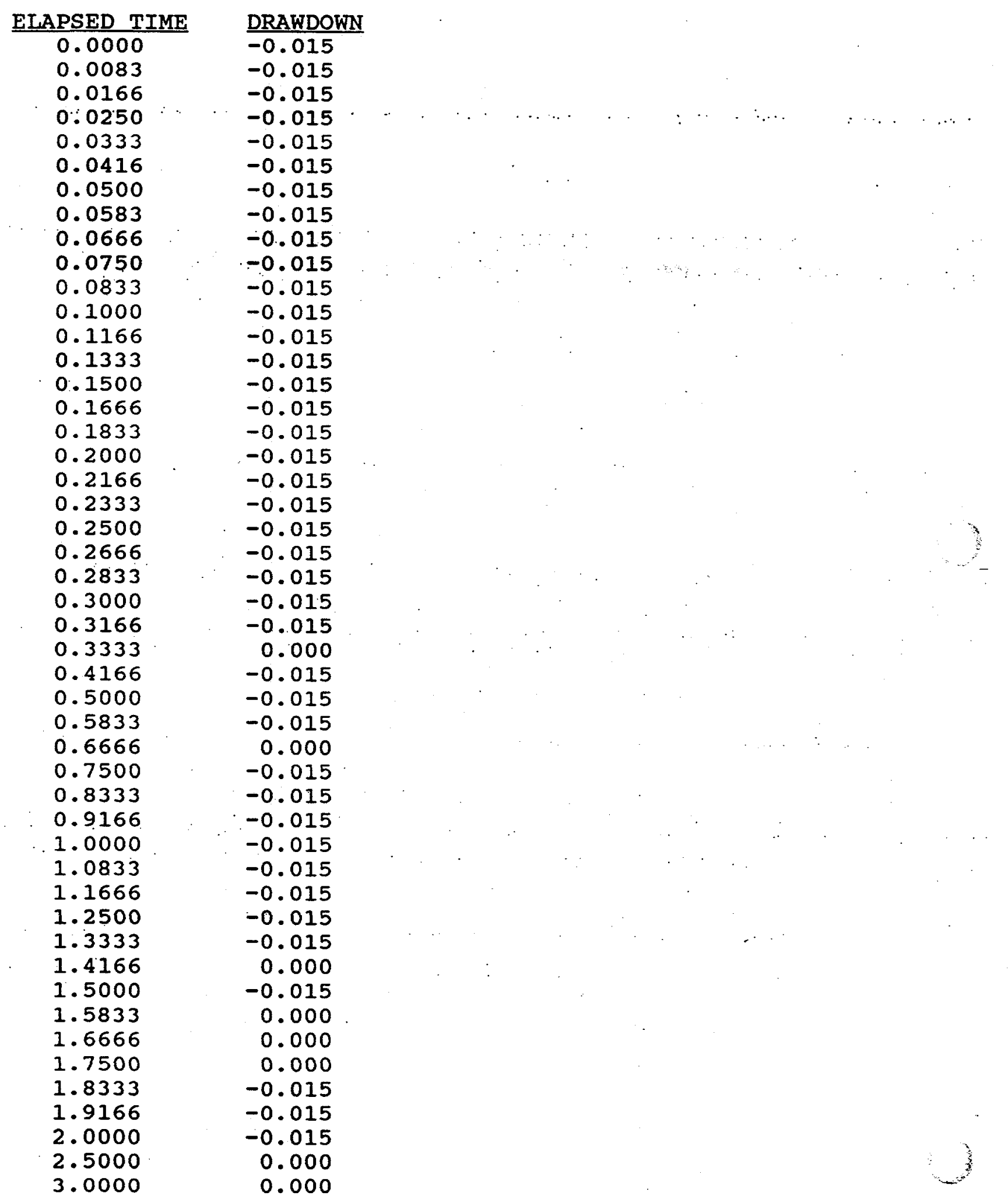




$\begin{array}{lr}3.5000 & 0.000 \\ 4.0000 & -0.015 \\ 4.5000 & -0.015 \\ 5.0000 & -0.031 \\ 5.5000 & -0.031 \\ 6.0000 & -0.031 \\ 6.5000 & -0.031 \\ 7.0000 & -0.031 \\ 7.5000 & -0.047 \\ 8.0000 & -0.047 \\ 8.5000 & -0.031 \\ 9.0000 & -0.031 \\ 9.5000 & -0.031 \\ 10.0000 & -0.031 \\ 12.0000 & 0.000 \\ 14.0000 & -0.015 \\ 16.0000 & -0.031 \\ 18.0000 & -0.031 \\ 20.0000 & -0.031 \\ 22.0000 & -0.031 \\ 24.0000 & -0.031 \\ 26.0000 & -0.047 \\ 28.0000 & -0.047 \\ 30.0000 & -0.063 \\ 32.0000 & -0.063 \\ 34.0000 & -0.063 \\ 36.0000 & -0.047 \\ 38.0000 & -0.063 \\ 40.0000 & -0.063 \\ 42.0000 & -0.063 \\ 44.0000 & -0.063 \\ 46.0000 & -0.063 \\ 48.0000 & -0.063 \\ 50.0000 & -0.078 \\ 52.0000 & -0.063 \\ 54.0000 & -0.063 \\ 56.0000 & -0.078 \\ 58.0000 & -0.063 \\ 60.0000 & -0.078 \\ 62.0000 & -0.047 \\ 64.0000 & -0.063 \\ 66.0000 & -0.063 \\ 68.0000 & -0.063 \\ 70.0000 & -0.047 \\ 72.0000 & -0.063 \\ 74.0000 & -0.063 \\ 76.0000 & -0.078 \\ 78.0000 & -0.078 \\ 80.0000 & -0.063 \\ 82.0000 & -0.078 \\ 84.0000 & -0.078 \\ 86.0000 & -0.063 \\ 88.0000 & -0.078 \\ 90.0000 & \end{array}$




$\begin{array}{ll}92.0000 & -0.063 \\ 94.0000 & -0.063 \\ 96.0000 & -0.063 \\ 98.0000 & -0.063 \\ 100.000 & -0.047 \\ 110.000 & -0.078 \\ 120.000 & -0.047 \\ 130.000 & -0.078 \\ 140.000 & -0.063 \\ 150.000 & -0.078 \\ 160.000 & -0.078 \\ 170.000 & -0.094 \\ 180.000 & -0.063 \\ 190.000 & -0.078 \\ 200.000 & -0.078 \\ 210.000 & -0.078 \\ 220.000 & -0.078 \\ 230.000 & -0.078 \\ 240.000 & -0.078 \\ 250.000 & -0.078 \\ 260.000 & -0.078 \\ 270.000 & -0.078 \\ 280.000 & -0.078 \\ 290.000 & -0.078 \\ 300.000 & -0.078 \\ 310.000 & -0.094 \\ 320.000 & -0.078 \\ 330.000 & -0.094 \\ 340.000 & -0.078 \\ 350.000 & -0.078 \\ 360.000 & -0.078 \\ 370.000 & -0.078 \\ 380.000 & -0.094 \\ 390.000 & -0.078 \\ 400.000 & -0.078 \\ 410.000 & -0.078 \\ 420.000 & -0.078 \\ 430.000 & -0.078 \\ 440.000 & -0.094 \\ 450.000 & -0.078 \\ 460.000 & -0.078 \\ 470.000 & -0.078 \\ 480.000 & -0.078 \\ 490.000 & -0.078 \\ 500.000 & -0.078 \\ 510.000 & -0.078 \\ 520.000 & -0.078 \\ 530.000 & -0.078 \\ 540.000 & -0.078 \\ 550.000 & -0.078 \\ 560.000 & -0.078 \\ 570.000 & -0.078 \\ 580.000 & -0.078 \\ 590.000 & -0.078\end{array}$




$\begin{array}{ll}600.000 & -0.063 \\ 610.000 & -0.078 \\ 620.000 & -0.078 \\ 630.000 & -0.078 \\ 640.000 & -0.078 \\ 650.000 & -0.063 \\ 660.000 & -0.063 \\ 670.000 & -0.063 \\ 680.000 & -0.063 \\ 690.000 & -0.063 \\ 700.000 & -0.063 \\ 710.000 & -0.063 \\ 720.000 & -0.063 \\ 730.000 & -0.063 \\ 740.000 & -0.063 \\ 750.000 & -0.063 \\ 760.000 & -0.063 \\ 770.000 & -0.063 \\ 780.000 & -0.063 \\ 790.000 & -0.063 \\ 800.000 & -0.063 \\ 810.000 & -0.063 \\ 820.000 & -0.063 \\ 830.000 & -0.063 \\ 840.000 & -0.063 \\ 850.000 & -0.063 \\ 860.000 & -0.063 \\ 870.000 & -0.063 \\ 880.000 & -0.063 \\ 890.000 & -0.063 \\ 900.000 & -0.063 \\ 910.000 & -0.063 \\ 920.000 & -0.063 \\ 930.000 & -0.063 \\ 940.000 & -0.063 \\ 950.000 & -0.063 \\ 960.000 & -0.063 \\ 970.000 & -0.063 \\ 980.0 .00 & -0.063 \\ 990.000 & -0.063 \\ 1000.00 & -0.063 \\ 1100.00 & -0.047 \\ 1200.00 & -0.047 \\ E N D & \\ & \end{array}$




\section{HSB $84 C$ \\ MAY 8,1990 \\ TEST TWO \\ DRAFDOKA DATA}

\begin{tabular}{|c|c|}
\hline ELAPSED TIME & DRAWDOWN \\
\hline 0.0000 & -0.031 \\
\hline 0.0083 & -0.031 \\
\hline 0.0166 & -0.031 \\
\hline 0.0250 & -0.031 \\
\hline 0.0333 & -0.031 \\
\hline 0.0 .416 & -0.031 \\
\hline 0.0500 & -0.015 \\
\hline 0.0583 & -0.015 \\
\hline 0.0666 & -0.015 \\
\hline 0.0750 & -0.015 \\
\hline 0.0833 & -0.015 \\
\hline 0.1000 & -0.015 \\
\hline 0.1166 & -0.015 \\
\hline 0.1333 & -0.015 \\
\hline 0.1500 & -0.015 \\
\hline 0.1666 & -0.015 \\
\hline 0.1833 & -0.015 \\
\hline 0.2000 & -0.015 \\
\hline 0.2166 & -0.015 \\
\hline 0.2333 & -0.015 \\
\hline 0.2500 & -0.015 \\
\hline 0.2666 & 0.000 \\
\hline 0.2833 & 0.000 \\
\hline 0.3000 & 0.000 \\
\hline 0.3166 & 0.000 \\
\hline 0.3333 & 0.000 \\
\hline 0.4166 & 0.000 \\
\hline 0.5000 & 0.000 \\
\hline 0.5833 & 0.000 \\
\hline 0.6666 & 0.000 \\
\hline 0.7500 & 0.000 \\
\hline 0.8333 & 0.000 \\
\hline 0.9166 & 0.000 \\
\hline 1.0000 & 0.000 \\
\hline 1.0833 & 0.000 \\
\hline 1.1666 & 0.000 \\
\hline 1.2500 & 0.000 \\
\hline 1.3333 & 0.015 \\
\hline 1.4166 & 0.015 \\
\hline 1.5000 & 0.015 \\
\hline 1.5833 & 0.015 \\
\hline 1.6666 & 0.015 \\
\hline 1.7500 & 0.015 \\
\hline 1.8333 & 0.015 \\
\hline 1.9166 & 0.015 \\
\hline 2.0000 & 0.015 \\
\hline 2.5000 & 0.031 \\
\hline 3.0000 & 0.031 \\
\hline
\end{tabular}




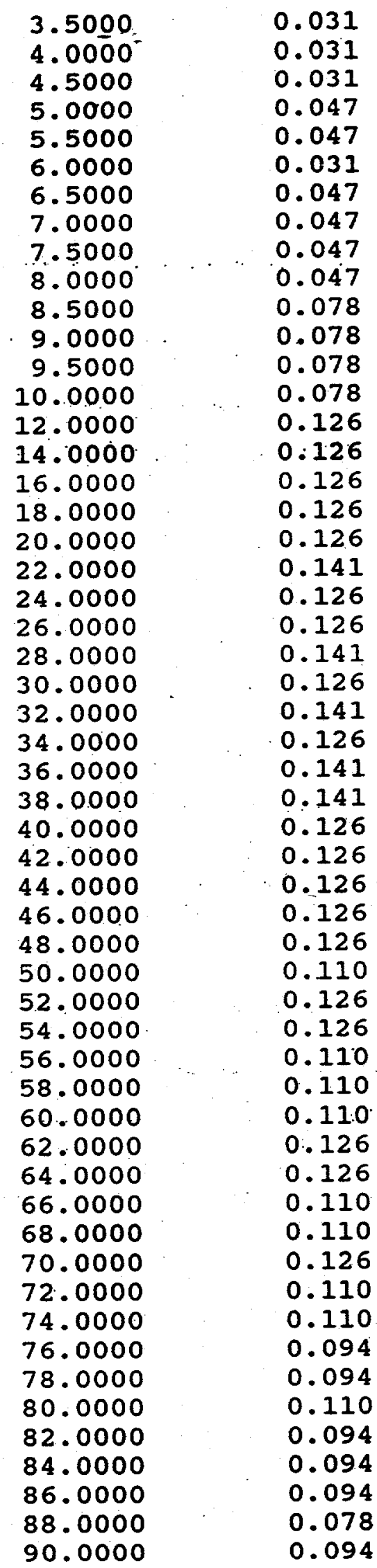




\begin{tabular}{|c|c|}
\hline $\begin{array}{l}92.0000 \\
94.0000 \\
96.0000 \\
98.0000 \\
100.000 \\
110.000 \\
120.000 \\
130.000 \\
140.000 \\
150.000 \\
160.000 \\
170.000 \\
180.000 \\
190.000 \\
200.000 \\
210.000 \\
220.000 \\
230.000 \\
240.000 \\
250.000 \\
260.000 \\
270.000 \\
280.000 \\
290.000 \\
300.000 \\
310.000 \\
320.000 \\
330.000 \\
340.000 \\
350.000 \\
360.000 \\
370.000 \\
380.000 \\
390.000 \\
400.000 \\
410.000 \\
420.000 \\
430.000 \\
440.000 \\
450.000 \\
460.000 \\
470.000 \\
480.000 \\
490.000 \\
500.000 \\
510.000 \\
520.000 \\
530.000 \\
540.000 \\
550.000 \\
560.000 \\
580.000\end{array}$ & $\begin{array}{r}0.094 \\
0.094 \\
0.094 \\
0.094 \\
0.094 \\
0.063 \\
0.047 \\
0.015 \\
0.015 \\
0.015 \\
0.015 \\
-0.015 \\
0.015 \\
0.000 \\
0.000 \\
0.000 \\
0.000 \\
0.000 \\
0.000 \\
0.000 \\
0.000 \\
0.000 \\
0.000 \\
-0.015 \\
0.000 \\
-0.015 \\
0.000 \\
-0.015 \\
-0.015 \\
-0.015 \\
-0.015 \\
-0.015 \\
-0.015 \\
-0.015 \\
-0.015 \\
-0.015 \\
-0.015 \\
-0.015 \\
-0.015 \\
-0.015 \\
-0.015 \\
-0.015 \\
-0.015 \\
-0.015 \\
-0.015 \\
-0.015 \\
-0.031 \\
-0.031 \\
-0.015 \\
-0.015 \\
-0.015 \\
-0.015 \\
0.015\end{array}$ \\
\hline
\end{tabular}




$\begin{array}{lr}600.000 & -0.015 \\ 610.000 & -0.015 \\ 620.000 & -0.015 \\ 630.000 & -0.015 \\ 640.000 & -0.015 \\ 650.000 & -0.015 \\ 660.000 & -0.015 \\ 670.000 & -0.015 \\ 680.000 & -0.015 \\ 690.000 & -0.015 \\ 700.000 & -0.015 \\ 710.000 & -0.015 \\ 720.000 & -0.015 \\ 730.000 & -0.015 \\ 740.000 & -0.015 \\ 750.000 & 0.000 \\ 760.000 & -0.015 \\ 770.000 & -0.015 \\ 780.000 & -0.015 \\ 790.000 & 0.000 \\ 800.000 & 0.000 \\ 810.000 & -0.015 \\ 820.000 & -0.015 \\ 830.000 & -0.015 \\ 840.000 & -0.015 \\ 850.000 & 0.000 \\ 860.000 & -0.015 \\ 870.000 & 0.000 \\ 880.000 & 0.000 \\ 890.000 & 0.000 \\ 900.000 & -0.015 \\ 910.000 & 0.000 \\ 920.000 & 0.000 \\ 930.000 & 0.000 \\ 940.000 & 0.000 \\ 950.000 & 0.000 \\ 960.000 & 0.000 \\ 970.000 & 0.000 \\ 980.000 & 0.000 \\ 990.000 & 0.000 \\ 1000.00 & 0.000 \\ 1100.00 & -0.078 \\ 1200.00 & -0.063 \\ N D & \\ & \end{array}$




\section{HSB 84D \\ MAY 9, 1990 \\ TEST TTO \\ RECOVERY DATA}

EIAPSED TIME

$\begin{array}{ll}0.0000 & 11.794 \\ 0.0083 & 11.794 \\ 0.0166 & 11.794 \\ 0.0250 & 11.794 \\ 0.0333 & 11.794 \\ 0.0416 & 11.794 \\ 0.0500 & 11.794 \\ 0.0583 & 11.794 \\ 0.0666 & 11.794 \\ 0.0750 & 11.794 \\ 0.0833 & 11.794 \\ 0.1000 & 11.794 \\ 0.1166 & 11.794 \\ 0.1333 & 11.794 \\ 0.1500 & 11.794 \\ 0.1666 & 11.794 \\ 0.1833 & 11.794 \\ 0.2000 & 11.794 \\ 0.2166 & 11.794 \\ 0.2333 & 11.794 \\ 0.2500 & 11.794 \\ 0.2666 & 11.794 \\ 0.2833 & 11.794 \\ 0.3000 & 11.794 \\ 0.3166 & 11.794 \\ 0.3333 & 11.794 \\ 0.4166 & 11.794 \\ 0.5000 & 11.794 \\ 0.5833 & 11.794 \\ 0.6666 & 11.794 \\ 0.7500 & 11.794 \\ 0.8333 & 11.794 \\ 0.9166 & 11.794 \\ 1.0000 & 11.794 \\ 1.0833 & 11.794 \\ 1.1666 & 11.794 \\ 1.2500 & 11.794 \\ 1.3333 & 11.794 \\ 1.4166 & 11.794 \\ 1.5000 & 11.794 \\ 1.5833 & 11.794 \\ 1.6666 & 11.794 \\ 1.7500 & 11.794 \\ 1.8333 & 11.794 \\ 1.9166 & 11.794 \\ 2.0000 & 11.794 \\ 2.5000 & 11.794 \\ 3.0000 & 11.794\end{array}$




\begin{tabular}{ll}
3.5000 & 11.794 \\
4.0000 & 11.794 \\
4.5000 & 11.794 \\
5.0000 & 11.794 \\
5.5000 & 11.794 \\
6.0000 & 11.794 \\
6.5000 & 11.794 \\
7.0000 & 11.794 \\
7.5000 & 11.794 \\
8.0000 & 1.11794 \\
8.5000 & 11.794 \\
9.0000 & 11.794 \\
9.5000 & 11.794 \\
10.0000 & 11.794 \\
12.0000 & 11.794 \\
14.0000 & 11.794 \\
16.0000 & 11.794 \\
18.0000 & 11.794 \\
20.0000 & 11.794 \\
22.0000 & 11.794 \\
24.0000 & 11.794 \\
26.0000 & 11.794 \\
28.0000 & 11.794 \\
30.0000 & 11.794 \\
32.0000 & 11.794 \\
34.0000 & 11.794 \\
36.0000 & 11.794 \\
38.0000 & 11.794 \\
40.0000 & 11.794 \\
42.0000 & 11.794 \\
44.0000 & 11.794 \\
46.0000 & 11.794 \\
48.0000 & 11.794 \\
50.0000 & 11.794 \\
52.0000 & 11.794 \\
54.0000 & 11.794 \\
56.0000 & 11.794 \\
58.0000 & 11.794 \\
60.0000 & 11.794 \\
62.0000 & 11.794 \\
64.0000 & 11.794 \\
66.0000 & 11.794 \\
68.0000 & 11.794 \\
70.0000 & 11.794 \\
72.0000 & 11.794 \\
74.0000 & 11.794 \\
76.0000 & 11.794 \\
78.0000 & 11.794 \\
80.0000 & 11.794 \\
82.0000 & 11.794 \\
84.0000 & 11.794 \\
86.0000 & 11.794 \\
88.0000 & 11.794 \\
90.0000 & 11.794 \\
& \\
\hline & \\
\hline
\end{tabular}




$\begin{array}{ll}92.0000 & 11.794 \\ 94.0000 & 11.794 \\ 96.0000 & 11.794 \\ 98.0000 & 11.794 \\ 100.000 & 11.794 \\ 110.000 & 11.794 \\ 120.000 & 11.794 \\ 130.000 & 11.794 \\ 140.000 & 11.794 \\ 150.000 & 11.794 \\ 160.000 & 11.794 \\ 170.000 & 11.794 \\ 180.000 & 11.794 \\ 190.000 & 11.794 \\ 200.000 & 11.794 \\ 210.000 & 11.794 \\ 220.000 & 11.794 \\ 230.000 & 11.794 \\ 240.000 & 11.794 \\ 250.000 & 11.794 \\ 260.000 & 11.794 \\ 270.000 & 11.794 \\ 280.000 & 11.794 \\ 290.000 & 11.794 \\ 300.000 & 11.794 \\ 310.000 & 11.794 \\ 320.000 & 11.794 \\ 330.000 & 11.794 \\ 340.000 & 11.794 \\ 350.000 & 11.794 \\ 360.000 & 11.794 \\ 370.000 & 11.794 \\ 380.000 & 11.794 \\ 390.000 & 11.794 \\ 400.000 & 11.794 \\ 410.000 & 11.794 \\ 420.000 & 11.794 \\ 430.000 & 11.794 \\ 440.000 & 11.794 \\ 450.000 & 11.794 \\ 460.000 & 11.794 \\ 470.000 & 11.794 \\ 480.000 & 11.794 \\ 490.000 & 11.794 \\ 500.000 & 11.794 \\ 510.000 & 11.794 \\ 520.000 & 11.794 \\ 530.000 & 11.794 \\ 540.000 & 11.794 \\ 550.000 & 11.794 \\ 560.000 & 11.794 \\ 570.000 & 11.794 \\ 580.000 & 11.794 \\ 590.000 & \end{array}$




$\begin{array}{ll}600.000 & 11.794 \\ 610.000 & 11.794 \\ 620.000 & 11.794 \\ 630.000 & 11.794 \\ 640.000 & 11.794 \\ 650.000 & 11.794 \\ 660.000 & 11.794 \\ 670.000 & 11.794 \\ 680.000 & 11.794 \\ 690.000 & 11.794 \\ 700.000 & 11.794 \\ 710.000 & 11.794 \\ 720.000 & 11.794 \\ 730.000 & 11.794 \\ 740.000 & 11.794 \\ 750.000 & 11.794 \\ 760.000 & 11.794 \\ 770.000 & 11.794 \\ 780.000 & 11.794 \\ 790.000 & 11.794 \\ 800.000 & 11.794 \\ 810.000 & 11.794 \\ 820.000 & 11.794 \\ 830.000 & 11.794 \\ 840.000 & 11.794 \\ 850.000 & 11.794 \\ 860.000 & 11.794 \\ 870.000 & 11.794 \\ 880.000 & 11.794 \\ 890.000 & 11.794 \\ 900.000 & 11.794 \\ 910.000 & 11.794 \\ 920.000 & 11.794 \\ 930.000 & 11.794 \\ 940.000 & 11.794 \\ 950.000 & 11.794 \\ 960.000 & 11.794 \\ 970.000 & 11.794 \\ 980.000 & 11.794 \\ 990.000 & 11.794 \\ 1000.00 & 11.794 \\ 1100.00 & 11.794 \\ 1200.00 & 11.794 \\ E N D & \\ & \end{array}$




\section{HSB 69 \\ MAY 8, 19̄90 \\ TEST TWO \\ DRAWDOWN DATA}

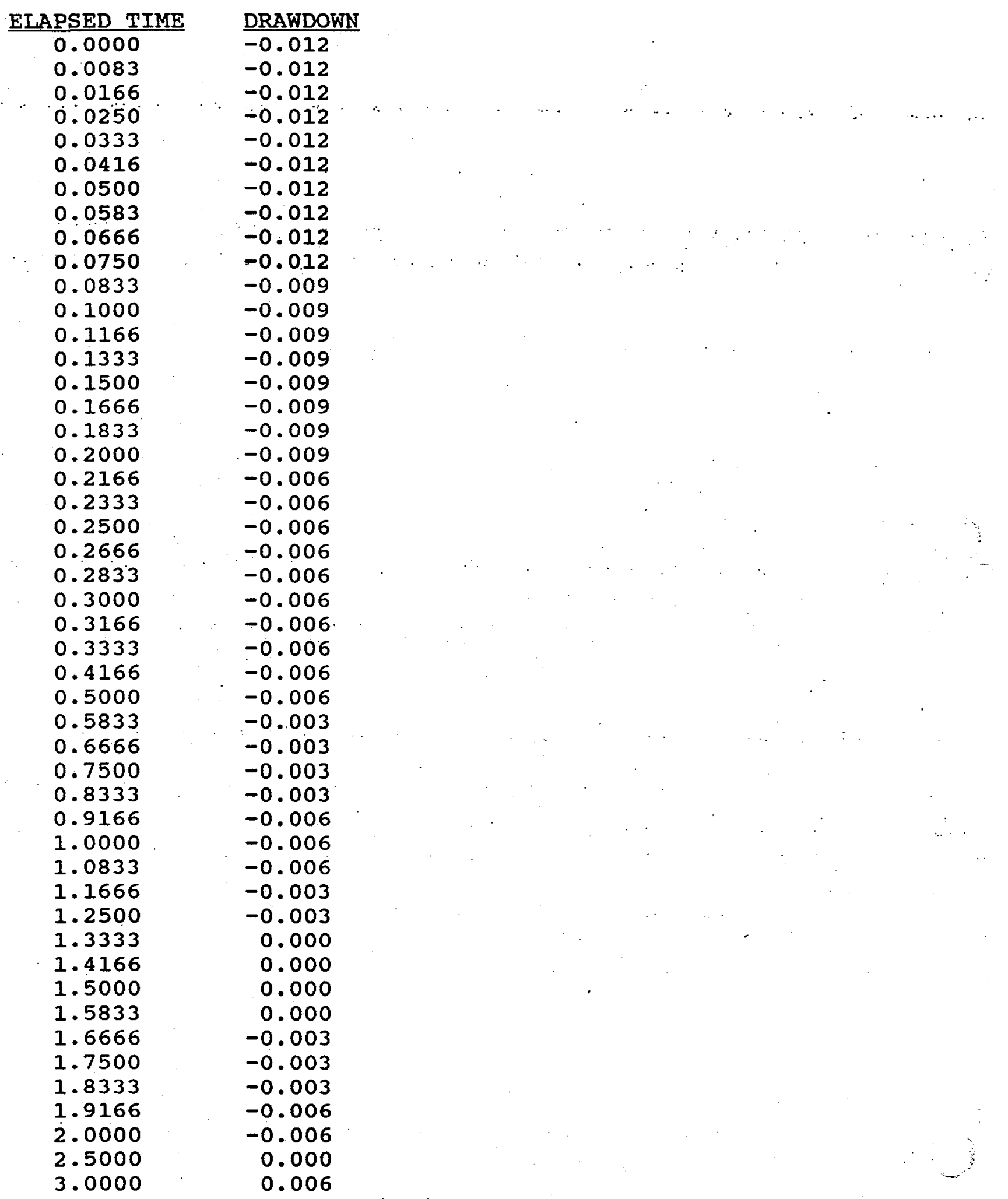




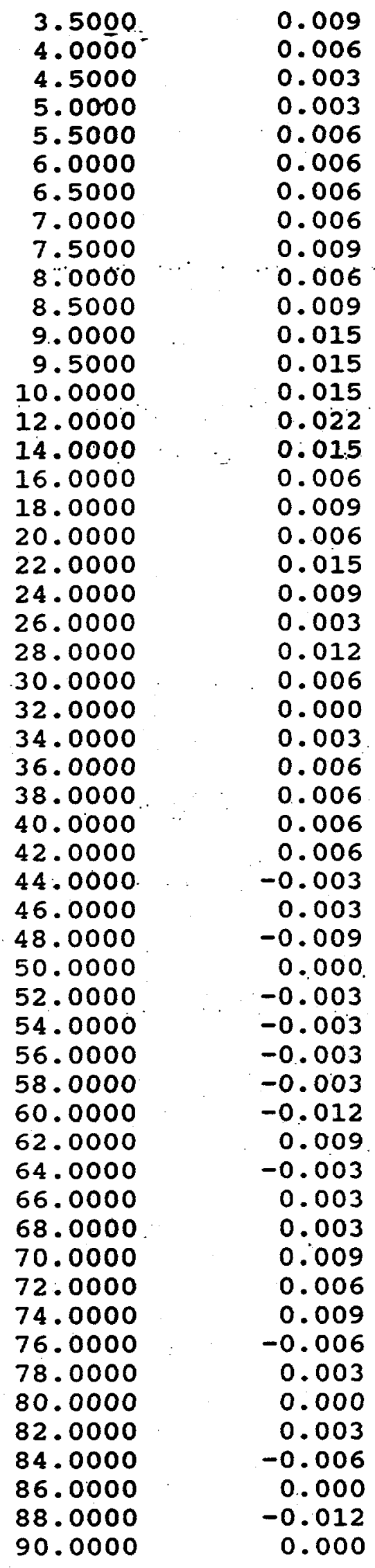




$\begin{array}{ll}92.0000 & -0.003 \\ 94.0000 & 0.000 \\ 96.0000 & 0.000 \\ 98.0000 & 0.006 \\ 100.000 & 0.009 \\ 110.000 & -0.015 \\ 120.000 & -0.009 \\ 130.000 & -0.018 \\ 140.000 & -0.015 \\ 150.000 & -0.012 \\ 160.000 & -0.012 \\ 170.000 & -0.034 \\ 180.000 & -0.015 \\ 190.000 & -0.022 \\ 200.000 & -0.022 \\ 210.000 & -0.031 \\ 220.000 & -0.028 \\ 230.000 & -0.025 \\ 240.000 & -0.028 \\ 250.000 & -0.028 \\ 260.000 & -0.025 \\ 270.000 & -0.028 \\ 280.000 & -0.028 \\ 290.000 & -0.025 \\ 300.000 & -0.022 \\ 310.000 & -0.028 \\ 320.000 & -0.025 \\ 330.000 & -0.031 \\ 340.000 & -0.025 \\ 350.000 & -0.028 \\ 360.000 & -0.028 \\ 370.000 & -0.025 \\ 380.000 & -0.025 \\ 390.000 & -0.025 \\ 400.000 & -0.025 \\ 410.000 & -0.028 \\ 420.000 & -0.028 \\ 430.000 & -0.028 \\ 440.000 & -0.031 \\ 450.000 & -0.028 \\ 460.000 & -0.031 \\ 470.000 & -0.028 \\ 480.000 & -0.028 \\ 490.000 & -0.028 \\ 500.000 & -0.028 \\ 510.000 & -0.025 \\ 520.000 & -0.025 \\ 530.000 & -0.025 \\ 540.000 & -0.022 \\ 550.000 & -0.022 \\ 560.000 & -0.022 \\ 570.000 & -0.018 \\ 580.000 & -0.018 \\ 590.000 & -0.018\end{array}$




\begin{tabular}{ll}
600.000 & -0.018 \\
610.000 & -0.018 \\
620.000 & -0.018 \\
630.000 & -0.018 \\
640.000 & -0.022 \\
650.000 & -0.018 \\
660.000 & -0.018 \\
670.000 & -0.022 \\
680.000 & -0.022 \\
690.000 & -0.022 \\
700.000 & -0.022 \\
710.000 & -0.022 \\
720.000 & -0.022 \\
730.000 & -0.022 \\
740.000 & -0.025 \\
750.000 & -0.025 \\
760.000 & -0.025 \\
770.000 & -0.025 \\
780.000 & -0.028 \\
790.000 & -0.028 \\
800.000 & -0.028 \\
810.000 & -0.031 \\
820.000 & -0.031 \\
830.000 & -0.031 \\
840.000 & -0.034 \\
850.000 & -0.034 \\
860.000 & -0.037 \\
870.000 & -0.037 \\
880.000 & -0.037 \\
890.000 & -0.040 \\
900.000 & -0.040 \\
910.000 & -0.037 \\
920.000 & -0.040 \\
930.000 & -0.040 \\
940.000 & -0.040 \\
950.000 & -0.040 \\
960.000 & -0.044 \\
970.000 & -0.044 \\
980.000 & -0.040 \\
990.000 & -0.040 \\
100.0 .00 & -0.040 \\
1100.00 & -0.034 \\
1200.00 & -0.028 \\
\hline 0 & \\
\hline & \\
\hline 70
\end{tabular}




\section{HSB 69A \\ MAY 8,1900 \\ TEST THO \\ DRATDOWN DATA}

\begin{tabular}{lc} 
ELAPSED TIME & DRAWDOWN \\
\hline 0.0000 & 0.050 \\
0.0083 & 0.053 \\
0.0166 & 0.053 \\
0.0250 & 0.053 \\
0.0333 & 0.053 \\
0.0416 & 0.056 \\
0.0500 & 0.056 \\
0.0583 & 0.056 \\
0.0666 & 0.056 \\
0.0750 & 0.056 \\
0.0833 & 0.056 \\
0.1000 & 0.059 \\
0.1166 & 0.059 \\
0.1333 & 0.059 \\
0.1500 & 0.059 \\
0.1666 & 0.059 \\
0.1833 & 0.063 \\
0.2000 & 0.063 \\
0.2166 & 0.063 \\
0.2333 & 0.063 \\
0.2500 & 0.066 \\
0.2666 & 0.066 \\
0.2833 & 0.066 \\
0.3000 & 0.066 \\
0.3166 & 0.066 \\
0.3333 & 0.066 \\
0.4166 & 0.069 \\
0.5000 & 0.072 \\
0.5833 & 0.075 \\
0.6666 & 0.078 \\
0.7500 & 0.085 \\
0.8333 & 0.091 \\
0.9166 & 0.101 \\
1.0000 & 0.110 \\
1.0833 & 0.119 \\
1.1666 & 0.132 \\
1.2500 & 0.145 \\
1.3333 & 0.157 \\
1.4166 & 0.170 \\
1.5000 & 0.183 \\
1.5833 & 0.198 \\
1.6666 & 0.211 \\
1.7500 & 0.227 \\
1.8333 & 0.243 \\
1.9166 & 0.255 \\
2.0000 & 0.271 \\
2.5000 & 0.372 \\
3.0000 & \\
& 0.467
\end{tabular}




\begin{tabular}{ll}
3.5000 & \\
4.0000 & 0.555 \\
4.5000 & 0.637 \\
5.0000 & 0.710 \\
5.5000 & 0.776 \\
6.0000 & 0.839 \\
6.5000 & 0.896 \\
7.0000 & 0.950 \\
7.5000 & 1.000 \\
8.0000 & 1.044 \\
8.5000 & 1.085 \\
9.0000 & 1.130 \\
9.5000 & 1.167 \\
10.0000 & 1.202 \\
12.0000 & 1.234 \\
14.0000 & 1.344 \\
16.0000 & 1.429 \\
18.0000 & 1.496 \\
20.0000 & 1.565 \\
22.0000 & 1.622 \\
24.0000 & 1.679 \\
26.0000 & 1.729 \\
28.0000 & 1.770 \\
30.0000 & 1.821 \\
32.0000 & 1.859 \\
34.0000 & 1.890 \\
36.0000 & 1.922 \\
38.0000 & 1.957 \\
40.0000 & 1.988 \\
42.0000 & 2.010 \\
44.0000 & 2.036 \\
46.0000 & 2.058 \\
48.0000 & 2.086 \\
50.0000 & 2.099 \\
52.0000 & 2.121 \\
54.0000 & 2.140 \\
56.0000 & 2.162 \\
58.0000 & 2.184 \\
60.0000 & 2.203 \\
62.0000 & 2.212 \\
64.0000 & 2.238 \\
66.0000 & 2.250 \\
68.0000 & 2.272 \\
70.0000 & 2.288 \\
72.0000 & 2.307 \\
74.0000 & 2.320 \\
76.0000 & 2.335 \\
78.0000 & 2.342 \\
80.0000 & 2.364 \\
82.0000 & 2.376 \\
84.0000 & 2.389 \\
86.0000 & 2.395 \\
88.0000 & \\
90.0000 & \\
& 2.418 \\
\hline &
\end{tabular}




$\begin{array}{ll}92.0000 & 2.446 \\ 94.0000 & 2.459 \\ 96.0000 & 2.468 \\ 98.0000 & 2.484 \\ 100.000 & 2.496 \\ 110.000 & 2.519 \\ 120.000 & 2.566 \\ 130.000 & 2.597 \\ 140.000 & 2.638 \\ 150.000 & 2.670 \\ 160.000 & 2.698 \\ 170.000 & 2.721 \\ 180.000 & 2.755 \\ 190.000 & 2.784 \\ 200.000 & 2.809 \\ 210.000 & 2.831 \\ 220.000 & 2.859 \\ 230.000 & 2.885 \\ 240.000 & 2.900 \\ 250.000 & 2.919 \\ 260.000 & 2.942 \\ 270.000 & 2.967 \\ 280.000 & 2.986 \\ 290.000 & 2.995 \\ 300.000 & 3.020 \\ 310.000 & 3.033 \\ 320.000 & 3.052 \\ 330.000 & 3.068 \\ 340.000 & 3.087 \\ 350.000 & 3.087 \\ 360.000 & 3.090 \\ 370.000 & 3.090 \\ 380.000 & 3.093 \\ 390.000 & 3.106 \\ 400.000 & 3.102 \\ 410.000 & 3.096 \\ 420.000 & 3.084 \\ 430.000 & 3.080 \\ 440.000 & 3.077 \\ 450.000 & 3.074 \\ 460.000 & 3.068 \\ 470.000 & 3.065 \\ 480.000 & 3.052 \\ 490.000 & 3.017 \\ 500.000 & 2.992 \\ 510.000 & 2.995 \\ 520.000 & 3.008 \\ 530.000 & 3.017 \\ 540.000 & 3.030 \\ 550.000 & 3.052 \\ 560.000 & 3.147 \\ 570.000 & \end{array}$




$\begin{array}{ll}600.000 & 3.166 \\ 610.000 & 3.194 \\ 620.000 & 3.204 \\ 630.000 & 3.216 \\ 640.000 & 3.229 \\ 650.000 & 3.235 \\ 660.000 & 3.248 \\ 670.000 & 3.248 \\ 680.000 & 3.241 \\ 690.000 & 3.232 \\ 700.000 & 3.226 \\ 710.000 & 3.226 \\ 720.000 & 3.219 \\ 730.000 & 3.254 \\ 740.000 & 3.311 \\ 750.000 & 3.339 \\ 760.000 & 3.346 \\ 770.000 & 3.339 \\ 780.000 & 3.339 \\ 790.000 & 3.333 \\ 800.000 & 3.339 \\ 810.000 & 3.330 \\ 820.000 & 3.323 \\ 830.000 & 3.330 \\ 840.000 & 3.327 \\ 850.000 & 3.317 \\ 860.000 & 3.311 \\ 870.000 & 3.311 \\ 880.000 & 3.320 \\ 890.000 & 3.327 \\ 900.000 & 3.311 \\ 910.000 & 3.295 \\ 920.000 & 3.282 \\ 930.000 & 3.279 \\ 940.000 & 3.282 \\ 950.000 & 3.279 \\ 960.000 & 3.286 \\ 970.000 & 3.295 \\ 980.000 & 3.298 \\ 990.000 & 3.292 \\ 1000.00 & 3.292 \\ 1100.00 & 3.418 \\ 1200.00 & 3.560\end{array}$




\section{HSB 84 A \\ MAY 9, 1990 \\ TEST TTO \\ RECOVERY DATA}

\begin{tabular}{cc} 
ELAPSED TIME & DRAWDOWN \\
\hline 0.0000 & 30.367 \\
0.0083 & 29.010 \\
0.0166 & 28.300 \\
0.0250 & 27.306 \\
0.0333 & 26.391 \\
0.0416 & 25.539 \\
0.0500 & 24.640 \\
0.0583 & 23.804 \\
0.0666 & 23.000 \\
0.0750 & 22.148 \\
0.0833 & 21.406 \\
0.1000 & 19.892 \\
0.1166 & 18.551 \\
0.1333 & 17.194 \\
0.1500 & 15.932 \\
0.1666 & 15.033 \\
0.1833 & 13.960 \\
0.2000 & 13.077 \\
0.2166 & 12.257 \\
0.2333 & 11.531 \\
0.2500 & 10.869 \\
0.2666 & 10.269 \\
0.2833 & 9.733 \\
0.3000 & 9.291 \\
0.3166 & 8.865 \\
0.3333 & 8.487 \\
0.4166 & 7.083 \\
0.5000 & 6.246 \\
0.5833 & 5.742 \\
0.6666 & 5.347 \\
0.7500 & 5.111 \\
0.8333 & 4.890 \\
0.9166 & 4.700 \\
1.0000 & 4.543 \\
1.0833 & 4.432 \\
1.1666 & 4.306 \\
1.2500 & 4.227 \\
1.3333 & 4.148 \\
1.4166 & 4.054 \\
1.5000 & 3.959 \\
1.5833 & 3.912 \\
1.6666 & 3.849 \\
1.7500 & 3.801 \\
1.8333 & 3.722 \\
1.9166 & 3.691 \\
2.0000 & 3.644 \\
2.5000 & 3.423 \\
3.0000 & 3.249
\end{tabular}




\begin{tabular}{|c|c|}
\hline 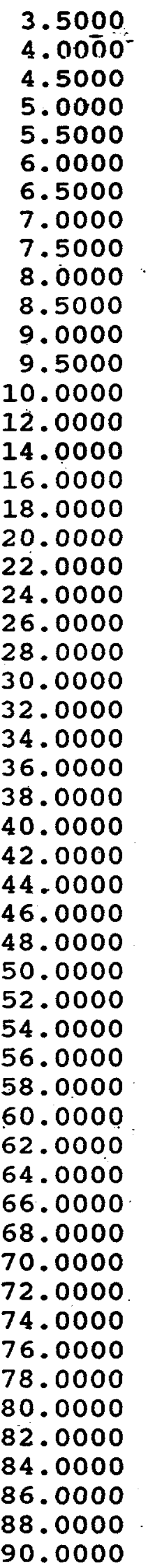 & $\begin{array}{l}3.123 \\
3.013 \\
2.918 \\
2.839 \\
2.792 \\
2.713 \\
2.665 \\
2.634 \\
2.587 \\
2.555 \\
2.539 \\
2.508 \\
2.476 \\
2.460 \\
2.382 \\
2.334 \\
2.287 \\
2.240 \\
2.176 \\
2.113 \\
2.066 \\
2.019 \\
1.971 \\
1.940 \\
1.893 \\
1.861 \\
1.829 \\
1.798 \\
1.766 \\
1.751 \\
1.719 \\
1.687 \\
1.672 \\
1.640 \\
1.624 \\
1.609 \\
1.577 \\
1.561 \\
1.545 \\
1.530 \\
1.514 \\
1.498 \\
1.482 \\
1.467 \\
1.451 \\
1.431 \\
1.403 \\
1.388 \\
1.372 \\
1.356 \\
1.340 \\
1.340\end{array}$ \\
\hline
\end{tabular}




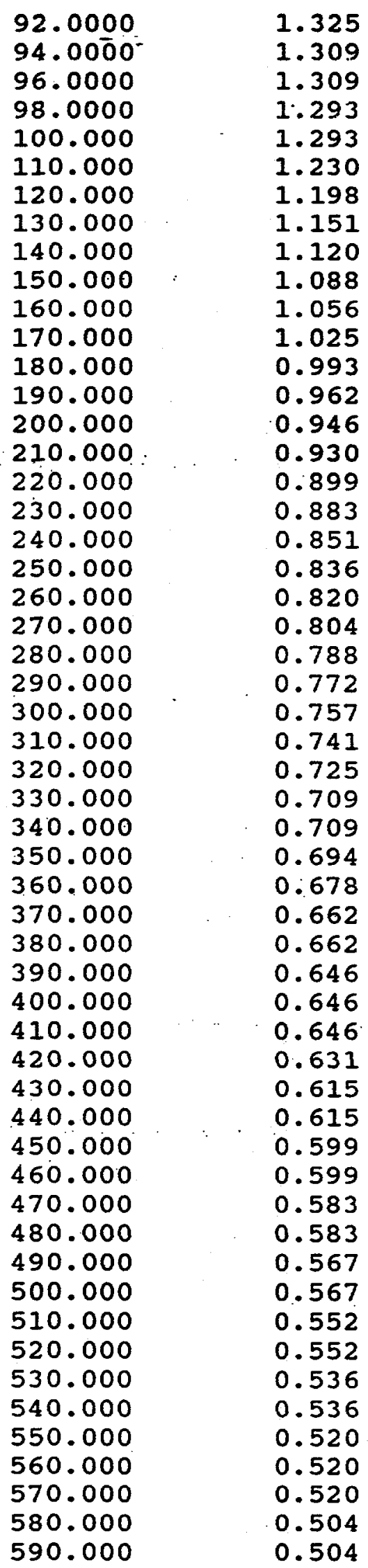




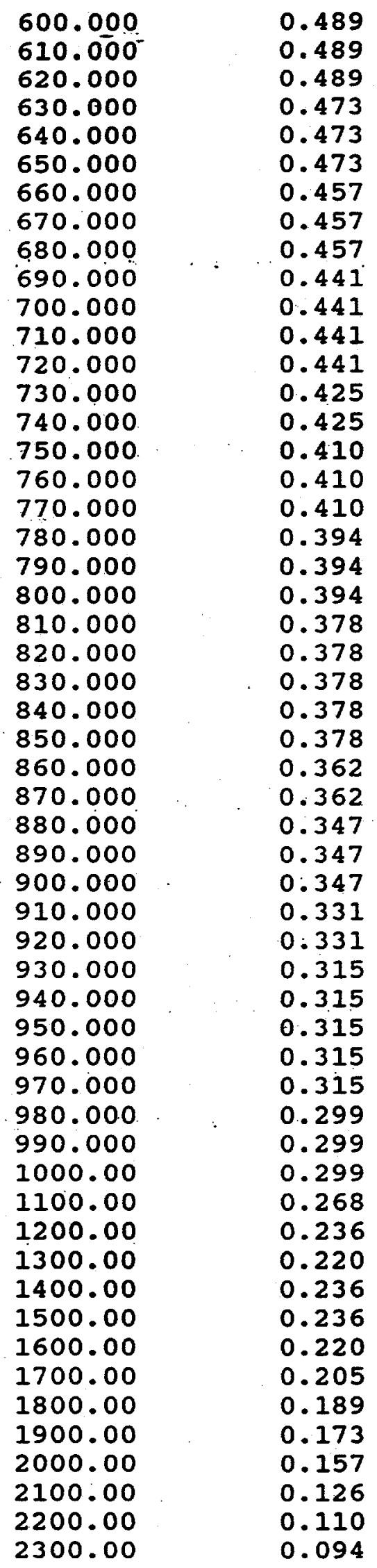




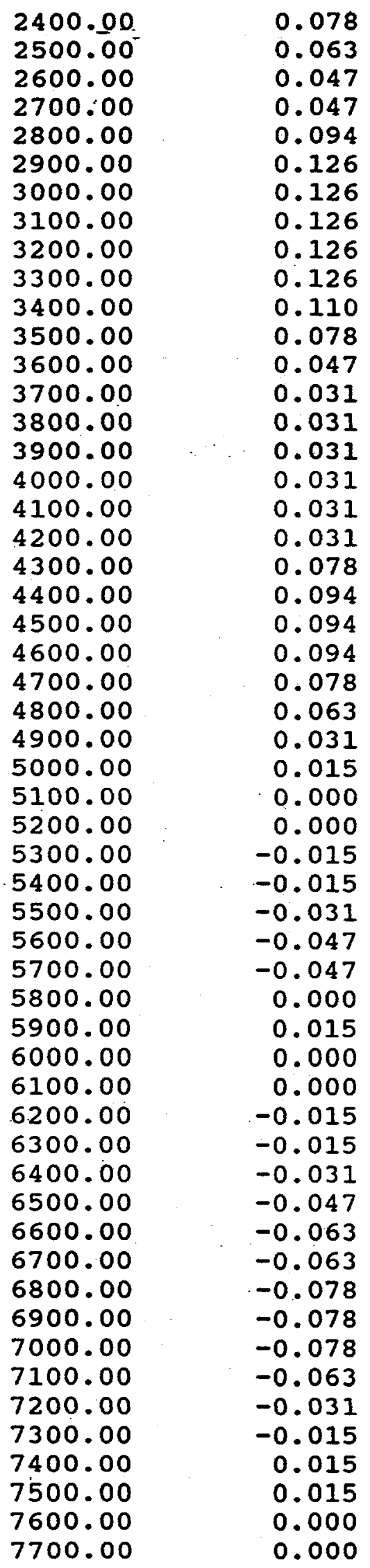




$\begin{array}{ll}7800.00 & -0.015 \\ 7900.00 & -0.031 \\ 8000.00 & -0.047 \\ 8100.00 & -0.047 \\ 8200.00 & -0.047 \\ 8300.00 & -0.047 \\ 8400.00 & -0.063 \\ 8500.00 & -0.031 \\ 8600.00 & 0.000 \\ 8700.00 & 60.749 \\ 8800.00 & 60.844 \\ 8900.00 & 67.485 \\ 9000.00 & 67.485 \\ 9100.00 & 67.485 \\ 9200.00 & 67.485 \\ 9300.00 & 67.485 \\ 9400.00 & 67.485 \\ 9500.00 & 67.485 \\ 9600.00 & 67.485 \\ 9700.00 & 67.485 \\ 9800.00 & 67.485 \\ 9900.00 & 67.485 \\ 10000.0 & 67.485 \\ 10500.0 & 67.485 \\ 11000.0 & 67.485 \\ 11500.0 & 67.485 \\ 12000.0 & 67.485 \\ 12500.0 & 67.485 \\ 13000.0 & 67.485 \\ 13500.0 & 67.485 \\ 14000.0 & 67.485 \\ 14500.0 & 67.485 \\ 15000.0 & 67.485 \\ 15500.0 & 67.485 \\ 16000.0 & 67.485 \\ 16500.0 & 67.485 \\ 17000.0 & 67.485 \\ 17500.0 & 67.485 \\ 18000.0 & 67.485 \\ 18500.0 & 67.485 \\ 19000.0 & 67.485 \\ 19500.0 & 67.485 \\ 20000.0 & 67.485 \\ 20500.0 & 67.485 \\ 21000.0 & 67.485 \\ 21500.0 & 67.485 \\ 22000.0 & 67.485 \\ 22500.0 & 67.485 \\ 23000.0 & 67.485 \\ 23500.0 & 67.485 \\ 24000.0 & 67.485 \\ 24500.0 & 67.485 \\ 25000.0 & 67.485 \\ 25500.0 & 67.485\end{array}$




$\begin{array}{ll}26000.0 & 67.485 \\ 26500.0 & 67.485 \\ 27000.0 & 67.485 \\ 27500.0 & 67.485 \\ 28000.0 & 67.485 \\ 28500.0 & 67.485 \\ 29000.0 & 67.485 \\ 29500.0 & 67.485 \\ 30000.0 & 67.485 \\ 30500.0 & 67.485 \\ 31000.0 & 67.485 \\ 31500.0 & 67.485 \\ 32000.0 & 67.485 \\ 32500.0 & 67.485 \\ 33000.0 & 67.485 \\ 33500.0 & 67.485 \\ 34000.0 & 67.485 \\ 34500.0 & 67.485 \\ 35000.0 & 67.485 \\ 35500.0 & 67.485 \\ 36000.0 & 67.485 \\ 36500.0 & 67.485 \\ 37000.0 & 67.485 \\ 37500.0 & 67.485 \\ 38000.0 & 67.485 \\ 38500.0 & 67.485 \\ 39000.0 & 67.485 \\ 39500.0 & 67.485 \\ 40000.0 & 67.485 \\ 40500.0 & 67.485 \\ 41000.0 & 67.485 \\ 41500.0 & 67.485\end{array}$

END 


\section{HSB $84 B$ \\ MAY 9, 1990 \\ TEST TTO \\ RECOVERY DATA}

\section{ELAPSED TIME}

0.0000

0.0083

0.0166

0.0250

0.0333

0.0416

0.0500

0.0583

0.0666

0.0750

0.0833

0.1000

0.1166

0.1333

0.1500

0.1666

0.1833

0.2000

0.2166

0.2333

0.2500

0.2666

0.2833

0.3000

0.3166

0.3333

0.4166

0.5000

0.5833

0.6666

0.7500

0.8333

0.9166

1.0000

1.0833

1.1666

1.2500

1.3333

1.4166

1.5000

1.5833

1.6666

1.7500

1.8333

1.9166

2.0000

2.5000

3.0000

DRAWDOWN

$-0.047$

$-0.063$

$-0.047$

$-0.047$

$-0.063$

$-0.063$

$-0.047$

$-0.063$

$-0.047$

$-0.063$

$-0.047$

$-0.047$

$-0.047$

$-0.047$

$-0.047$

$-0.047$

$-0.047$

$-0.047$

$-0.047$

$-0.063$

$-0.047$

$-0.047$

$-0.047$

$-0.047$

$-0.047$

$-0.047$

$-0.047$

$-0.063$

$-0.047$

$-0.063$

$-0.047$

$-0.047$

$-0.047$

$-0.047$

$-0.047$

$-0.047$

$-0.047$

$-0.047$

$-0.047$

$-0.047$

$-0.047$

$-0.047$

$-0.047$

$-0.047$

$-0.047$

$-0.047$

$-0.047$

$-0.047$ 


\begin{tabular}{|c|c|c|}
\hline $\begin{array}{l}3.5000 \\
4.0000 \\
4.5000 \\
5.0000 \\
5.5000 \\
6.0000 \\
6.5000 \\
7.0000 \\
7.5000 \\
8.0000 \\
8.5000 \\
9.0000 \\
9.5000 \\
10.0000 \\
12.0000 \\
14.0000 \\
16.0000 \\
18.0000 \\
20.0000 \\
22.0000 \\
24.0000 \\
26.0000 \\
28.0000 \\
30.0000 \\
32.0000 \\
34.0000 \\
36.0000 \\
38.0000 \\
40.0000 \\
42.0000 \\
44.0000 \\
46.0000 \\
48.0000 \\
50.0000 \\
52.0000 \\
54.0000 \\
56.0000 \\
58.0000 \\
60.0000 \\
62.0000 \\
64.0000 \\
66.0000 \\
68.0000 \\
70.0000 \\
72.0000 \\
74.0000 \\
76.0000 \\
78.0000 \\
80.0000 \\
82.0000 \\
84.0000 \\
86.0000 \\
88.0000 \\
90.0000\end{array}$ & $=$ & $\begin{array}{l}-0.031 \\
-0.031 \\
-0.031 \\
-0.031 \\
-0.031 \\
-0.031 \\
-0.015 \\
-0.015 \\
-0.015 \\
-0.015 \\
-0.015 \\
-0.015 \\
-0.015 \\
0.000 \\
0.015 \\
0.015 \\
0.031 \\
0.031 \\
0.031 \\
0.031 \\
0.047 \\
0.047 \\
0.047 \\
0.047 \\
0.047 \\
0.047 \\
0.047 \\
0.047 \\
0.063 \\
0.063 \\
0.063 \\
0.063 \\
0.063 \\
0.063 \\
0.063 \\
0.063 \\
0.063 \\
0.063 \\
0.063 \\
0.063 \\
0.063 \\
0.063 \\
0.0078 \\
0.063 \\
0.078 \\
0.0078 \\
0.063 \\
0.063 \\
0.063 \\
0.078\end{array}$ \\
\hline
\end{tabular}




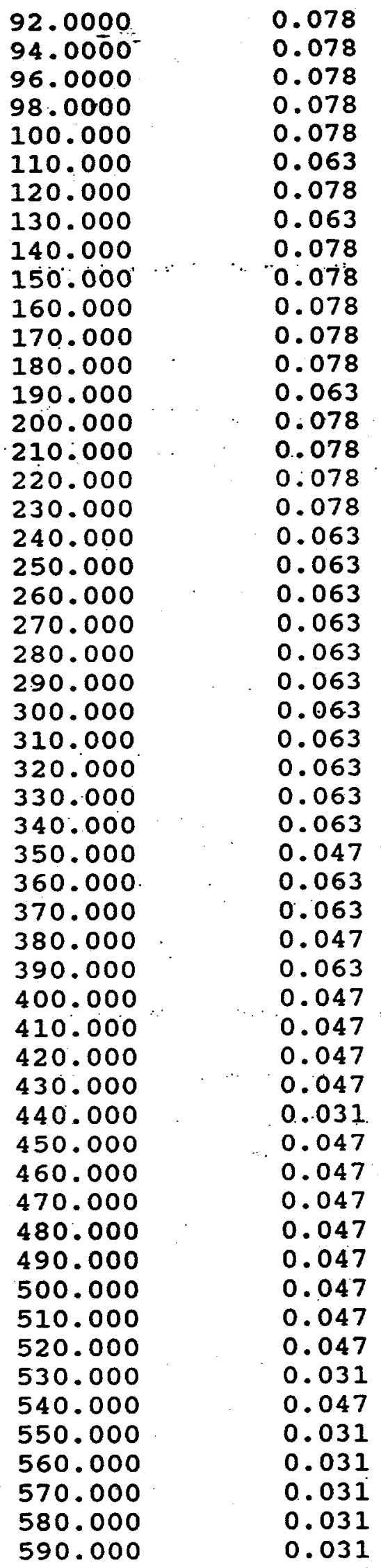




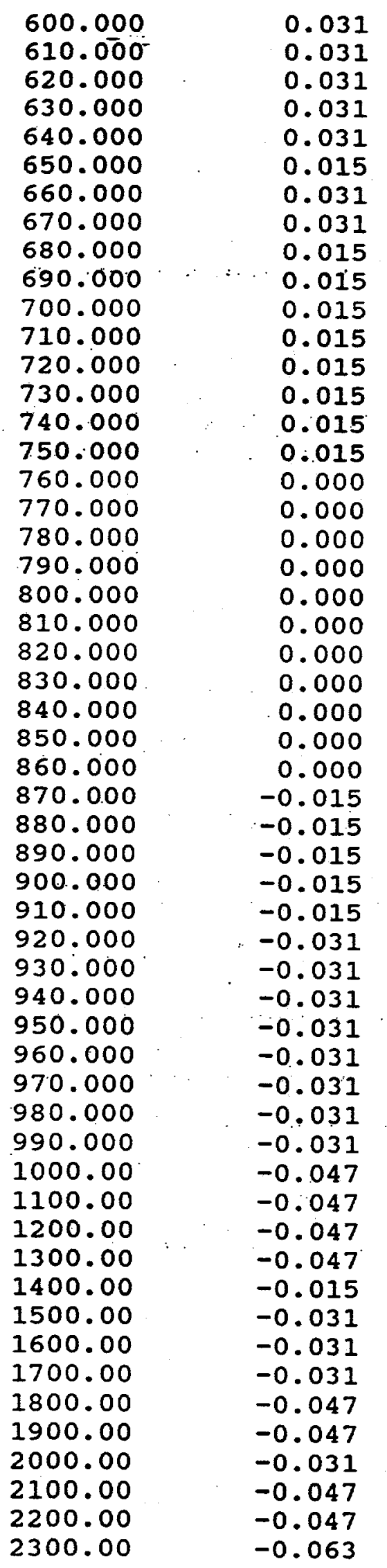




$\begin{array}{lr}2400.00 & -0.063 \\ 2500.00 & -0.063 \\ 2600.00 & -0.078 \\ 2700.00 & -0.078 \\ 2800.00 & -0.031 \\ 2900.00 & -0.015 \\ 3000.00 & 0.000 \\ 3100.00 & 0.000 \\ 3200.00 & -0.015 \\ 3300.00 & -0.015 \\ 3400.00 & -0.015 \\ 3500.00 & -0.031 \\ 3600.00 & -0.047 \\ 3700.00 & -0.047 \\ 3800.00 & -0.047 \\ 3900.00 & -0.047 \\ 4000.00 & -0.047 \\ 4100.00 & -0.047 \\ 4200.00 & -0.031 \\ 4300.00 & 0.000 \\ 4400.00 & 0.015 \\ 4500.00 & 0.000 \\ 4600.00 & 0.015 \\ 4700.00 & 0.000 \\ 4800.00 & 0.000 \\ 4900.00 & -0.015 \\ 5000.00 & -0.031 \\ 5100.00 & -0.031 \\ 5200.00 & -0.031 \\ 5300.00 & -0.031 \\ 5400.00 & -0.031 \\ 5500.00 & -0.031 \\ 5600.00 & -0.047 \\ 5700.00 & -0.047 \\ 5800.00 & -0.047 \\ 5900.00 & -0.015 \\ 6000.00 & -0.015 \\ 6100.00 & -0.015 \\ 6200.00 & -0.015 \\ 6300.00 & -0.015 \\ 6400.00 & -0.015 \\ 6500.00 & -0.031 \\ 6600.00 & -0.031 \\ 6700.00 & -0.031 \\ 6800.00 & -0.031 \\ 6900.00 & -0.031 \\ 7000.00 & -0.031 \\ 7100.00 & -0.015 \\ 7200.00 & 0.000 \\ 7300.00 & 0.015 \\ 7400.00 & \\ 7500.00 & \\ 7600.00 & 0.000 \\ 7700.00 & \end{array}$




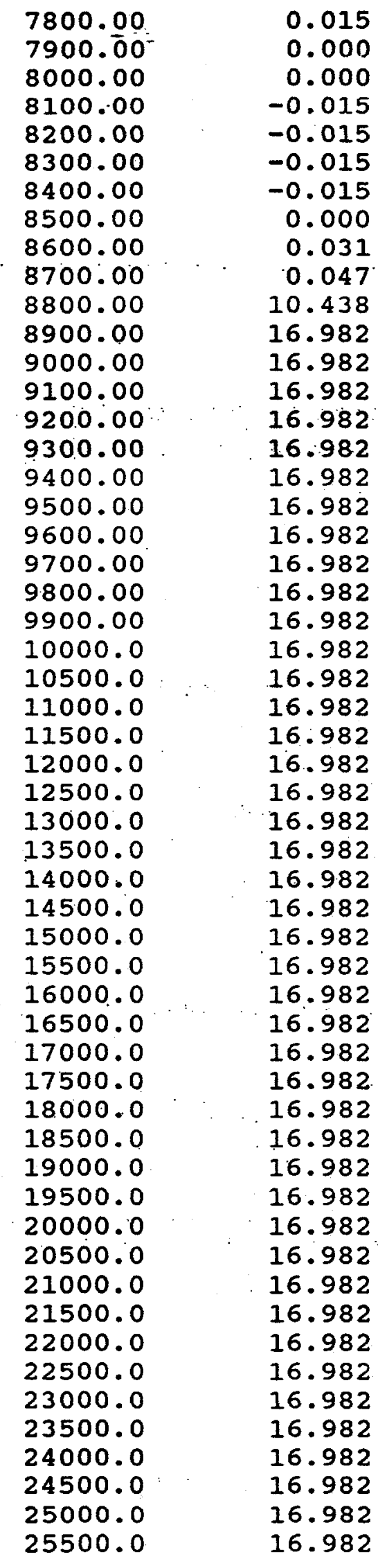




$\begin{array}{ll}26000.0 & 16.982 \\ 26500.0 & 16.982 \\ 27000.0 & 16.982 \\ 27500.0 & 16.982 \\ 28000.0 & 16.982 \\ 28500.0 & 16.982 \\ 29000.0 & 16.982 \\ 29500.0 & 16.982 \\ 30000.0 & 16.982 \\ 30500.0 & 16.982 \\ 31000.0 & 16.982 \\ 31500.0 & 16.982 \\ 32000.0 & 16.982 \\ 32500.0 & 16.982 \\ 33000.0 & 16.982 \\ 33500.0 & 16.982 \\ 34000.0 & 16.982 \\ 34500.0 & 16.982 \\ 35000.0 & 16.982 \\ 35500.0 & 16.982 \\ 36000.0 & 16.982 \\ 36500.0 & 16.982 \\ 37000.0 & 16.982 \\ 37500.0 & 16.982 \\ 38000.0 & 16.982 \\ 38500.0 & 16.982 \\ 39000.0 & 16.982 \\ 39500.0 & 16.982 \\ 40000.0 & 16.982 \\ 40500.0 & 16.982 \\ 41000.0 & 16.982 \\ 41500.0 & 16.982\end{array}$

END 


\section{HSB $84 \mathrm{C}$ \\ MAY $9,1 \overline{990}$ \\ TEST THO \\ RECOVERY DATA}

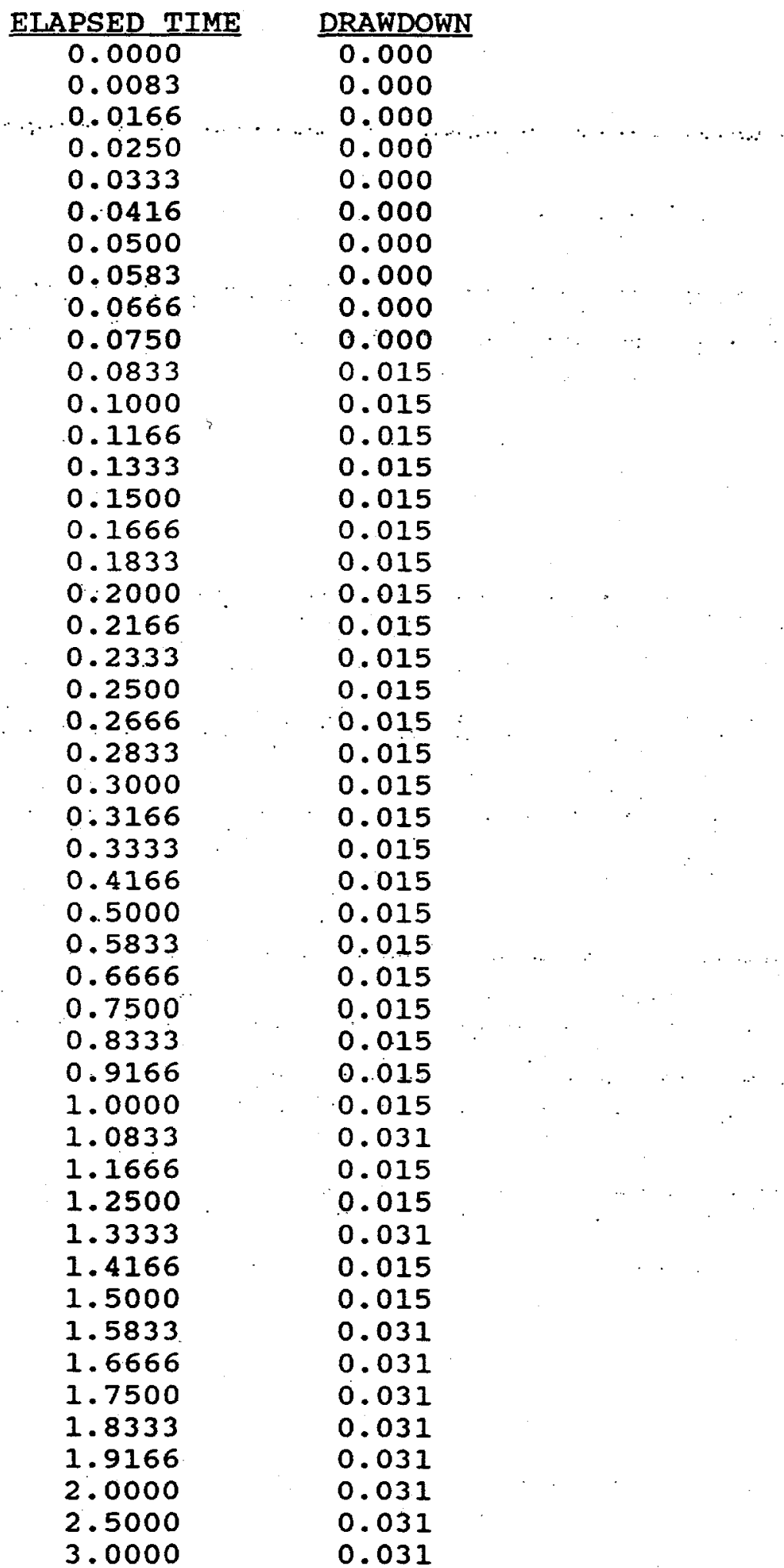




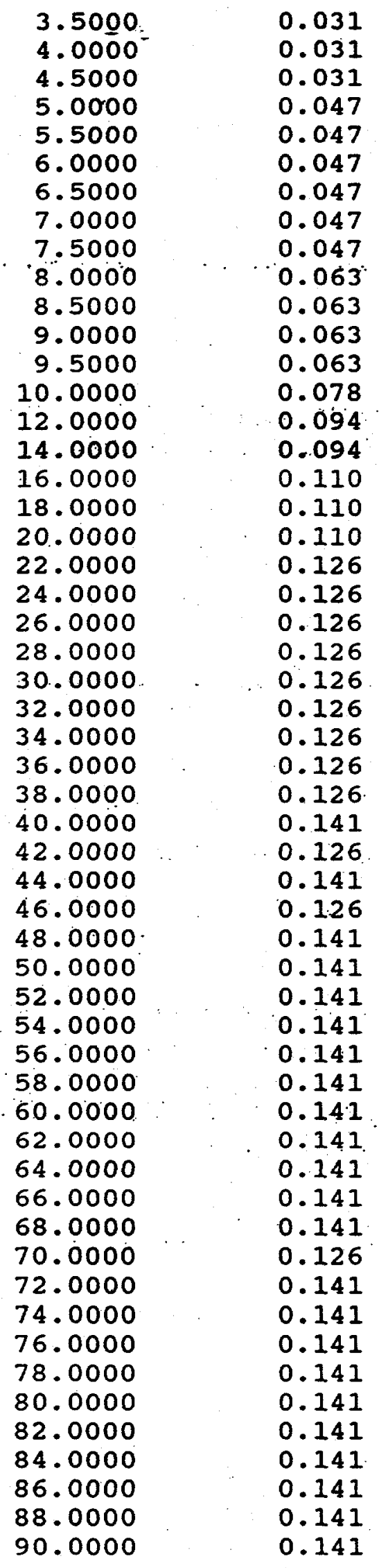




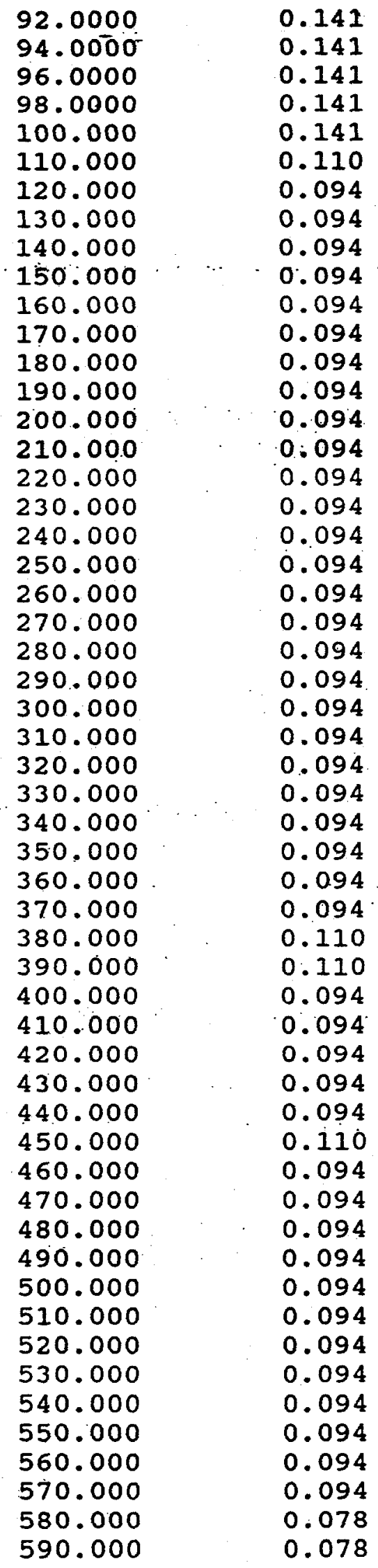




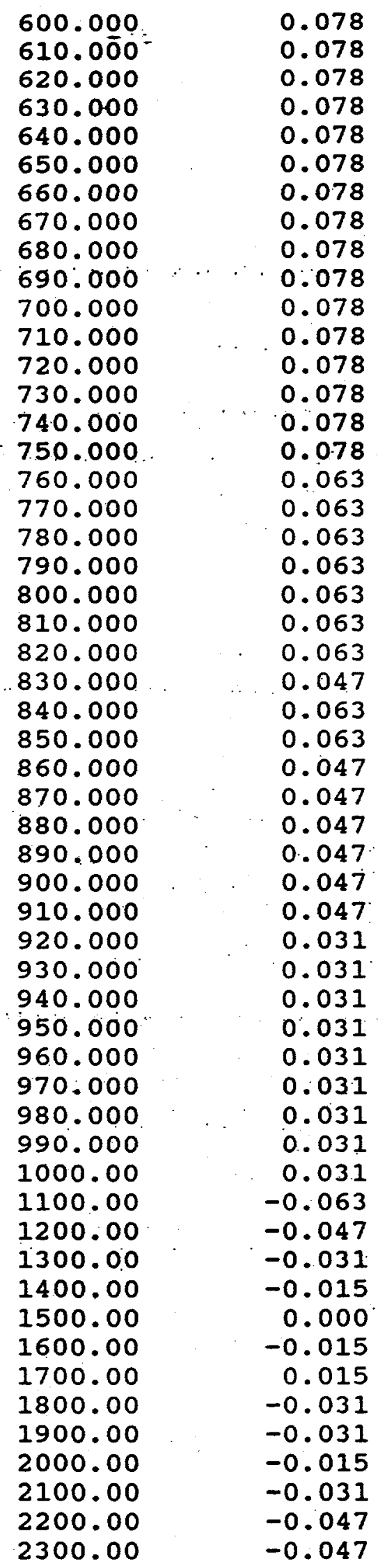




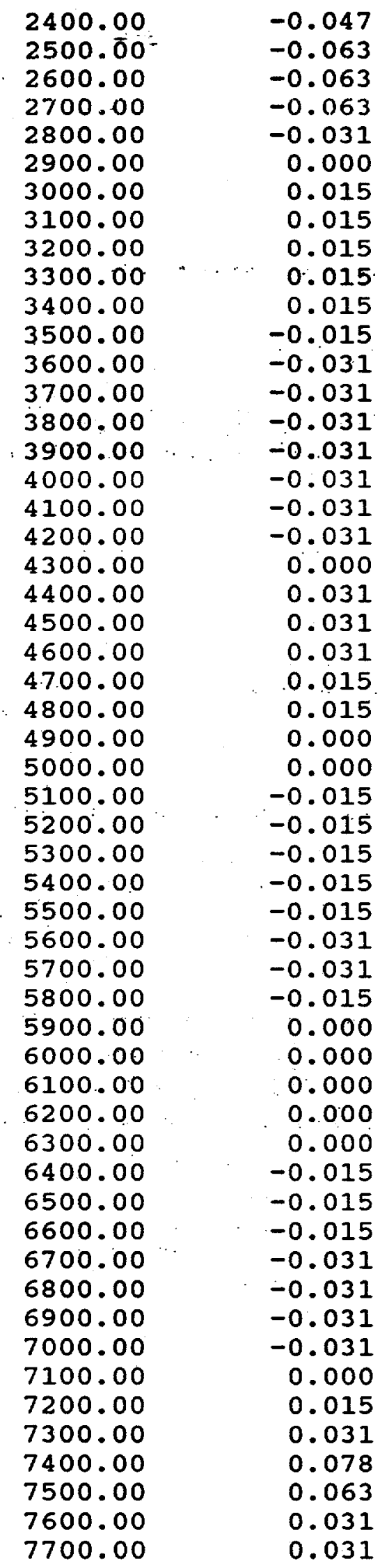




$\begin{array}{ll}7800.00 & 0.031 \\ 7900.00 & 0.015 \\ 8000.00 & 0.000 \\ 8100.00 & 0.000 \\ 8200.00 & 0.000 \\ 8300.00 & 0.000 \\ 8400.00 & 0.000 \\ 8500.00 & 0.015 \\ 8600.00 & 0.031 \\ 8700.00 & 0.047 \\ 8800.00 & 10.106 \\ 8900.00 & 16.901 \\ 9000.00 & 16.901 \\ 9100.00 & 16.901 \\ 9200.00 & 16.901 \\ 9300.00 & 16.901 \\ 9400.00 & 16.901 \\ 9500.00 & 16.901 \\ 9600.00 & 16.901 \\ 9700.00 & 16.901 \\ 9800.00 & 16.901 \\ 9900.00 & 16.901 \\ 10000.0 & 16.901 \\ 10500.0 & 16.901 \\ 11000.0 & 16.901 \\ 11500.0 & 16.901 \\ 12000.0 & 16.901 \\ 12500.0 & 16.901 \\ 13000.0 & 16.901 \\ 13500.0 & 16.901 \\ 14000.0 & 16.901 \\ 14500.0 & 16.901 \\ 15000.0 & 16.901 \\ 15500.0 & 16.901 \\ 16000.0 & 16.901 \\ 16500.0 & 16.901 \\ 17000.0 & 16.901 \\ 17500.0 & 16.901 \\ 18000.0 & 16.901 \\ 18500.0 & 16.901 \\ 19000.0 & 16.901 \\ 19500.0 & 16.901 \\ 20000.0 & 16.901 \\ 20500.0 & 16.901 \\ 21000.0 & 16.901 \\ 21500.0 & 16.901 \\ 22000.0 & 16.901 \\ 22500.0 & 16.901 \\ 23000.0 & 16.901 \\ 23500.0 & 16.901 \\ 245000.0 & 16.901 \\ 25000.0 & \end{array}$




$\begin{array}{ll}26000.0 & 16.901 \\ 26500.0 & 16.901 \\ 27000.0 & 16.901 \\ 27500.0 & 16.901 \\ 28000.0 & 16.901 \\ 28500.0 & 16.901 \\ 29000.0 & 16.901 \\ 29500.0 & 16.901 \\ 30000.0 & 16.901 \\ 30500.0 & 16.901 \\ 31000.0 & 16.901 \\ 31500.0 & 16.901 \\ 32000.0 & 16.901 \\ 32500.0 & 16.901 \\ 33000.0 & 16.901 \\ 33500.0 & 16.901 \\ 34000.0 & 16.901 \\ 34500.0 & 16.901 \\ 35000.0 & 16.901 \\ 35500.0 & 16.901 \\ 36000.0 & 16.901 \\ 36500.0 & 16.901 \\ 37000.0 & 16.901 \\ 37500.0 & 16.901 \\ 38000.0 & 16.901 \\ 38500.0 & 16.901 \\ 39000.0 & 16.901 \\ 39500.0 & 16.901 \\ 40000.0 & 16.901 \\ 40500.0 & 16.901 \\ 41000.0 & 16.901 \\ 41500.0 & 16.901 \\ 1 & \end{array}$

END 


\section{HSB 84D \\ MAY 9,1990 \\ TEST TWO \\ RECOVERY DATA}

ELAPSED TIME DRAWDOWN

0.0000

0.0083

0.0166

0.0250

0.0333

0.0416

0.0500

0.0583

0.0666

0.0750

0.0833

0.1000

0.1166

0.1333

0.1500

0.1666

0.1833

0.2000

0.2166

0.2333

0.2500

0.2666

0.2833

0.3000

0.31 .66

0.3333

0.4166

0.5000

0.5833

0.6666

0.7500

0.8333

0.9166

1.0000

1.0833

1. 1666

1.2500

1.3333

1.4166

1.5000

1.5833

1.6666

1.7500

1.8333

1.9166

2.0000

2.5000

3.0000
11.794

11.794

11.794

11.794

11.794

11.794

11.794

11.794

11.794

11.794

11.794

11.794

11.794

11.794

11.794

11.794

11.794

11.794

11.794

11.794

11.794

11.794

11.794

11.794

11.794

11.794

11.794

11.794

11.794

11.794

11.794

11.794

11.794

11.794

11.79 .4

11.794

11.794

11.794

11.794

11.794

11.794

11.794

11.794

11.794

11.794

11.794

11.794

11.794 


$\begin{array}{ll}3.5000 & 11.794 \\ 4.0000 & 11.794 \\ 4.5000 & 11.794 \\ 5.0000 & 11.794 \\ 5.5000 & 11.794 \\ 6.0000 & 11.794 \\ 6.5000 & 11.794 \\ 7.0000 & 11.794 \\ 7.5000 & 11.794 \\ 8.0000 & 11.794 \\ 8.5000 & 11.794 \\ 9.0000 & 11.794 \\ 9.5000 & 11.794 \\ 10.0000 & 11.794 \\ 12.0000 & 11.794 \\ 14.0000 & 11.794 \\ 16.0000 & 11.794 \\ 18.0000 & 11.794 \\ 20.0000 & 11.794 \\ 22.0000 & 11.794 \\ 24.0000 & 11.794 \\ 26.0000 & 11.794 \\ 28.0000 & 11.794 \\ 3.0 .0000 & 11.794 \\ 32.0000 & 11.794 \\ 34.0000 & 11.794 \\ 36.0000 & 11.794 \\ 38.0000 & 11.794 \\ 40.0000 & 11.794 \\ 42.0000 & 11.794 \\ 44.0000 & 11.794 \\ 46.0000 & 11.794 \\ 48.0000 & 11.794 \\ 50.0000 & 11.794 \\ 52.0000 & 11.794 \\ 54.0000 & 11.794 \\ 56.0000 & 11.794 \\ 58.0000 & 11.794 \\ 60.0000 & 11.794 \\ 62.0000 & 11.794 \\ 64.0000 & 11.794 \\ 66.0000 & 11.794 \\ 68.0000 & 11.794 \\ 70.0000 & 11.794 \\ 72.0000 & 11.794 \\ 74.0000 & 11.794 \\ 76.0000 & 11.794 \\ 78.0000 & 11.794 \\ 80.0000 & 11.794 \\ 82.0000 & 11.794 \\ 84.0000 & 11.794 \\ 86.0000 & 11.794 \\ 88.0000 & 11.794 \\ 90.0000 & 11.794 \\ & \end{array}$




\begin{tabular}{ll}
92.0000 & 11.794 \\
94.0000 & 11.794 \\
96.0000 & 11.794 \\
98.0000 & 11.794 \\
100.000 & 11.794 \\
110.000 & 11.794 \\
120.000 & 11.794 \\
130.000 & 11.794 \\
140.000 & 11.794 \\
150.000 & 11.794 \\
160.000 & 11.794 \\
170.000 & 11.794 \\
180.000 & 11.794 \\
190.000 & 11.794 \\
20.0 .000 & 11.794 \\
210.000 & 11.794 \\
220.000 & 11.794 \\
230.000 & 11.794 \\
240.000 & 11.794 \\
250.000 & 11.794 \\
260.000 & 11.794 \\
270.000 & 11.794 \\
280.000 & 11.794 \\
290.000 & 11.794 \\
300.000 & 11.794 \\
310.000 & 11.794 \\
320.000 & 11.794 \\
330.000 & 11.794 \\
340.000 & 11.794 \\
350.000 & 11.794 \\
360.000 & 11.794 \\
370.000 & 11.794 \\
380.000 & 11.794 \\
390.000 & 11.794 \\
400.000 & 11.794 \\
410.000 & 11.794 \\
420.000 & 11.794 \\
430.000 & 11.794 \\
440.000 & 11.794 \\
450.000 & 11.794 \\
460.000 & 11.794 \\
470.000 & 11.794 \\
480.000 & 11.794 \\
490.000 & 11.794 \\
500.000 & 11.794 \\
510.000 & 11.794 \\
520.000 & 11.794 \\
530.000 & 11.794 \\
540.000 & 11.794 \\
550.000 & 11.794 \\
560.000 & 11.794 \\
570.000 & 11.794 \\
580.000 & \\
590.000 & \\
\hline &
\end{tabular}




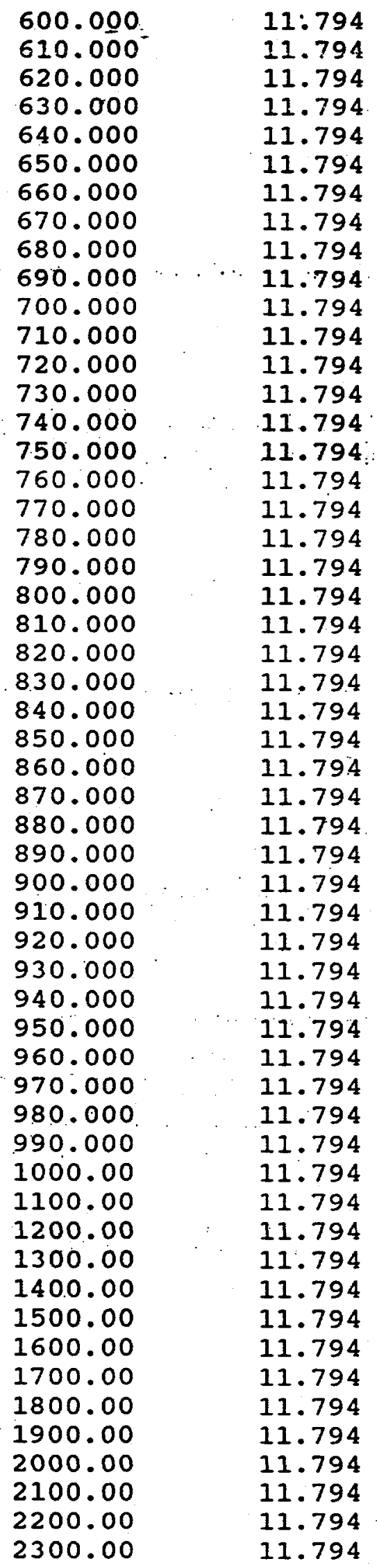




$\begin{array}{ll}2400.00 & 11.794 \\ 2500.00 & 11.794 \\ 2600.00 & 11.794 \\ 2700.00 & 11.794 \\ 2800.00 & 11.794 \\ 2900.00 & 11.794 \\ 3000.00 & 11.794 \\ 3100.00 & 11.794 \\ 3200.00 & 11.794 \\ 3300.00 & 11.794 \\ 3400.00 & 11.794 \\ 3500.00 & 11.794 \\ 3600.00 & 11.794 \\ 3700.00 & 11.794 \\ 3800.00 & 11.794 \\ 3900.00 & 11.794 \\ 4000.00 & 11.794 \\ 4100.00 & 11.794 \\ 4200.00 & 11.794 \\ 4300.00 & 11.794 \\ 4400.00 & 11.794 \\ 4500.00 & 11.794 \\ 4600.00 & 11.794 \\ 4700.00 & 11.794 \\ 4800.00 & 11.794 \\ 4900.00 & 11.794 \\ 5000.00 & 11.794 \\ 5100.00 & 11.794 \\ 5200.00 & 11.794 \\ 5300.00 & 11.794 \\ 5400.00 & 11.794 \\ 5500.00 & 11.794 \\ 5600.00 & 11.794 \\ 5700.00 & 11.794 \\ 5800.00 & 11.794 \\ 5900.00 & 11.794 \\ 6000.00 & 11.794 \\ 6100.00 & 11.794 \\ 6200.00 & 11.794 \\ 6300.00 & 11.794 \\ 6400.00 & 11.794 \\ 6500.00 & 11.794 \\ 6600.00 & 11.794 \\ 6700.00 & 11.794 \\ 6800.00 & 11.794 \\ 6900.00 & 11.794 \\ 7000.00 & 11.794 \\ 7100.00 & 11.794 \\ 7200.00 & 11.794 \\ 7300.00 & 11.794 \\ 7400.00 & 11.794 \\ 7500.00 & 11.794 \\ 7600.00 & 11.794 \\ 7700.00 & \end{array}$




$\begin{array}{ll}7800.00 & 11.794 \\ 7900.00 & 11.794 \\ 8000.00 & 11.794 \\ 8100.00 & 11.794 \\ 8200.00 & 11.794 \\ 8300.00 & 11.794 \\ 8400.00 & 11.794 \\ 8500.00 & 11.794 \\ 8600.00 & 11.794 \\ 8700.00 & 11.794 \\ 8800.00 & 11.794 \\ 8900.00 & 11.794 \\ 9000.00 & 11.794 \\ 9100.00 & 11.794 \\ 9200.00 & 11.794 \\ 9300.00 & 11.794 \\ 9400.00 & 11.794 \\ 9500.00 & 11.794 \\ 9600.00 & 11.794 \\ 9700.00 & 11.794 \\ 9800.00 & 11.794 \\ 9900.00 & 11.794 \\ 10000.0 & 11.794 \\ 10500.0 & 11.794 \\ 11000.0 & 11.794 \\ 11500.0 & 11.794 \\ 12000.0 & 11.794 \\ 12500.0 & 11.794 \\ 13000.0 & 11.794 \\ 13500.0 & 11.794 \\ 14000.0 & 11.794 \\ 14500.0 & 11.794 \\ 15000.0 & 11.794 \\ 15500.0 & 11.794 \\ 16000.0 & 11.794 \\ 16500.0 & 11.794 \\ 17000.0 & 11.794 \\ 17500.0 & 11.794 \\ 18000.0 & 11.794 \\ 18500.0 & 11.794 \\ 19000.0 & 11.794 \\ 19500.0 & 11.794 \\ 20000.0 & 11.794 \\ 20500.0 & 11.794 \\ 21000.0 & 11.794 \\ 21500.0 & 11.794 \\ 22000.0 & 11.794 \\ 22500.0 & 11.794 \\ 23000.0 & 11.794 \\ 23500.0 & 11.794 \\ 24000.0 & 1.794 \\ 25500.0 & \\ 25500.0 & 1194 \\ & \end{array}$




\begin{tabular}{ll}
26000.0 & 11.794 \\
26500.0 & 11.794 \\
27000.0 & 11.794 \\
27500.0 & 11.794 \\
28000.0 & 11.794 \\
28500.0 & 11.794 \\
29000.0 & 11.794 \\
29500.0 & 11.794 \\
30000.0 & 11.794 \\
30500.0 & 11.794 \\
31000.0 & 11.794 \\
31500.0 & 11.794 \\
32000.0 & 11.794 \\
32500.0 & 11.794 \\
33000.0 & 11.794 \\
33500.0 & 11.794 \\
34000.0 & 11.794 \\
34500.0 & 11.794 \\
35000.0 & 11.794 \\
35500.0 & 11.794 \\
36000.0 & 11.794 \\
36500.0 & 11.794 \\
37000.0 & 11.794 \\
37500.0 & 11.794 \\
38000.0 & 11.794 \\
38500.0 & 11.794 \\
39000.0 & 11.794 \\
39500.0 & 11.794 \\
40000.0 & 11.794 \\
40500.0 & 11.794 \\
41000.0 & 11.794 \\
41500.0 & 11.794 \\
\hline 0 &
\end{tabular}

END 


\section{HSB 69 \\ MAY 9,1990 \\ TEST TWO \\ RECOVERY DATA}

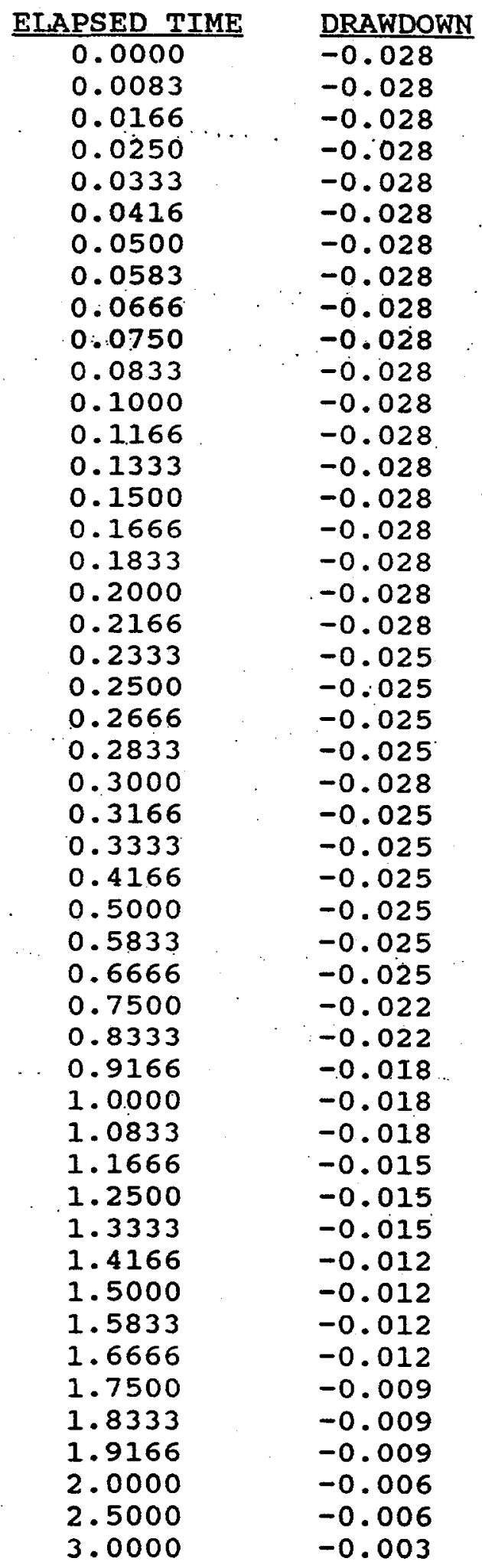




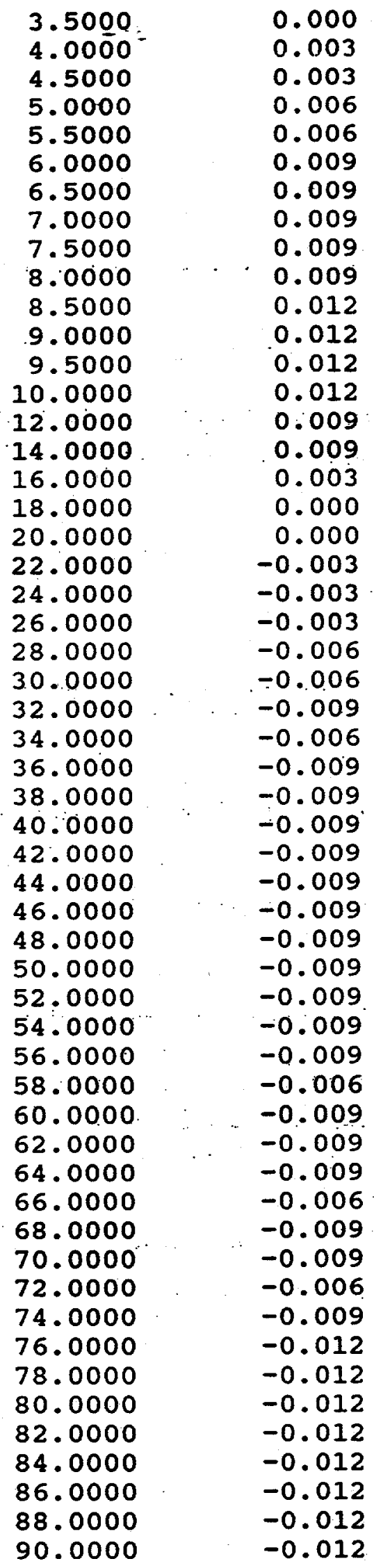




$\begin{array}{ll}92.0000 & -0.012 \\ 94.0000 & -0.015 \\ 96.0000 & -0.012 \\ 98.0000 & -0.015 \\ 100.000 & -0.012 \\ 110.000 & -0.025 \\ 120.000 & -0.034 \\ 130.000 & -0.031 \\ 140.000 & -0.028 \\ 150.000 & -0.031 \\ 160.000 & -0.031 \\ 170.000 & -0.034 \\ 180.000 & -0.034 \\ 190.000 & -0.037 \\ 200.000 & -0.034 \\ 210.000 & -0.031 \\ 220.000 & -0.031 \\ 230.000 & -0.031 \\ 240.000 & -0.034 \\ 250.000 & -0.037 \\ 260.000 & -0.034 \\ 270.000 & -0.037 \\ 280.000 & -0.037 \\ 290.000 & -0.040 \\ 300.000 & -0.044 \\ 310.000 & -0.040 \\ 320.000 & -0.044 \\ 330.000 & -0.044 \\ 340.000 & -0.044 \\ 350.000 & -0.047 \\ 360.000 & -0.047 \\ 370.000 & -0.047 \\ 380.000 & -0.044 \\ 390.000 & -0.047 \\ 400.000 & -0.050 \\ 410.000 & -0.056 \\ 420.000 & -0.053 \\ 430.000 & -0.053 \\ 44.0 .000 & -0.050 \\ 450.000 & -0.050 \\ 460.000 & -0.050 \\ 470.000 & -0.050 \\ 480.000 & -0.050 \\ 490.000 & -0.047 \\ 500.000 & -0.047 \\ 510.000 & -0.047 \\ 520.000 & -0.044 \\ 530.000 & -0.044 \\ 540.000 & -0.047 \\ 550.000 & -0.047 \\ 560.000 & -0.047 \\ 570.000 & -0.047 \\ 580.000 & -0.047 \\ 590.000 & -0.044 \\ & \end{array}$




$\begin{array}{ll}600.000 & -0.044 \\ 610.000 & -0.044 \\ 620.000 & -0.044 \\ 630.000 & -0.044 \\ 640.000 & -0.044 \\ 650.000 & -0.044 \\ 660.000 & -0.044 \\ 670.000 & -0.044 \\ 680.000 & -0.044 \\ 690.000 & -0.044 \\ 700.000 & -0.044 \\ 710.000 & -0.044 \\ 720.000 & -0.044 \\ 730.000 & -0.044 \\ 740.000 & -0.040 \\ 750.000 & -0.044 \\ 760.000 & -0.047 \\ 770.000 & -0.050 \\ 780.000 & -0.050 \\ 790.000 & -0.053 \\ 800.000 & -0.053 \\ 810.000 & -0.056 \\ 820.000 & -0.059 \\ 830.000 & -0.059 \\ 840.000 & -0.059 \\ 850.000 & -0.059 \\ 860.000 & -0.063 \\ 870.000 & -0.066 \\ 880.000 & -0.069 \\ 890.000 & -0.072 \\ 900.000 & -0.075 \\ 910.000 & -0.078 \\ 920.000 & -0.081 \\ 930.000 & -0.081 \\ 940.000 & -0.081 \\ 950.000 & -0.085 \\ 960.000 & -0.088 \\ 970.000 & -0.088 \\ 980.000 & -0.094 \\ 990.000 & -0.097 \\ 1000.00 & -0.097 \\ 1100.00 & -0.097 \\ 1200.00 & -0.091 \\ 1300.00 & -0.078 \\ 1400.00 & -0.069 \\ 1500.00 & -0.066 \\ 1600.00 & -0.072 \\ 1700.00 & -0.066 \\ 1800.00 & -0.078 \\ 1900.00 & -0.059 \\ 2000.00 & -0.050 \\ 2100.00 & -0.037 \\ 2200.00 & \\ 2300.00 & \end{array}$




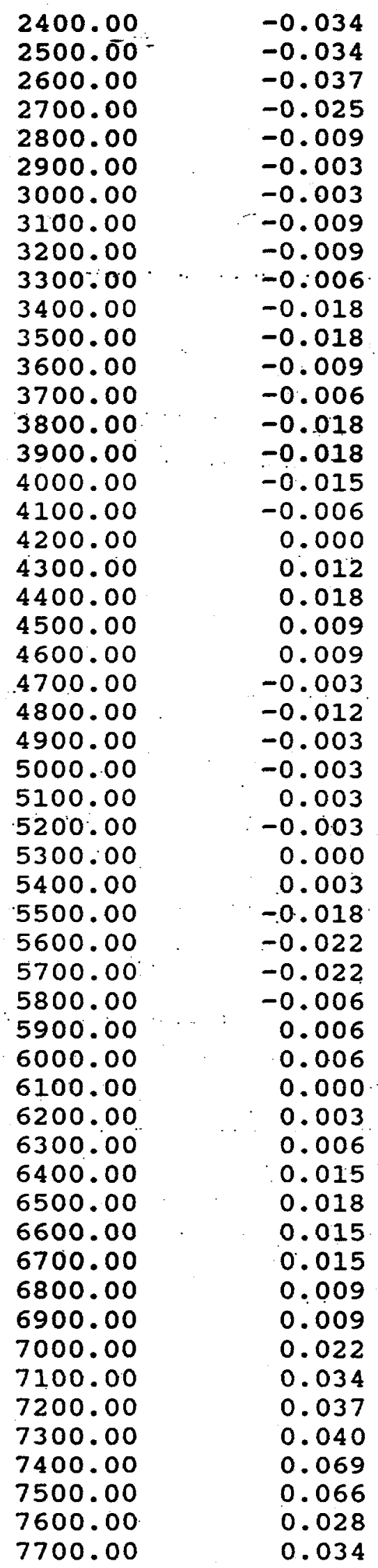




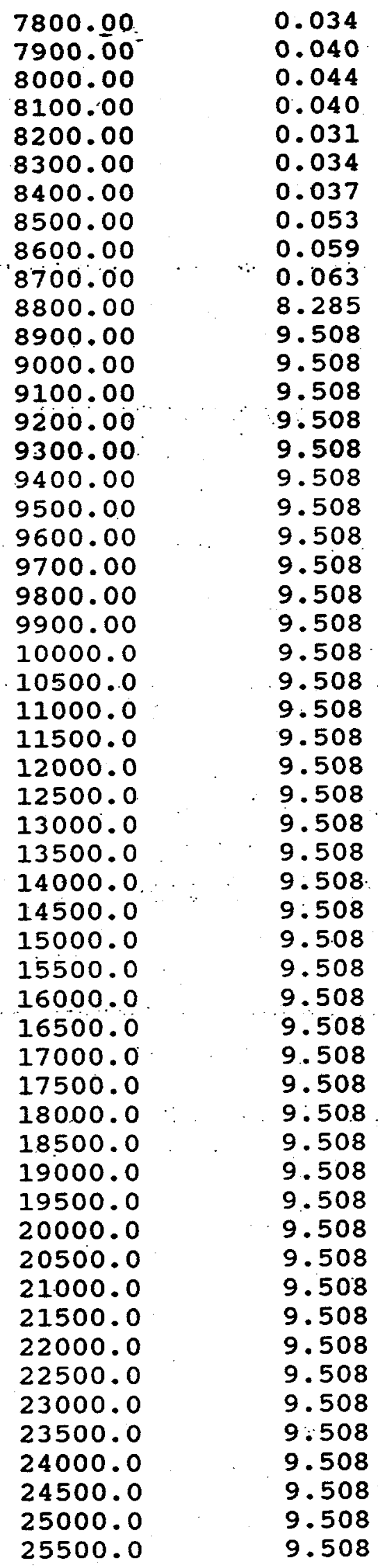




\begin{tabular}{ll}
26000.0 & 9.508 \\
26500.0 & 9.508 \\
27000.0 & 9.508 \\
27500.0 & 9.508 \\
28000.0 & 9.508 \\
28500.0 & 9.508 \\
29000.0 & 9.508 \\
29500.0 & 9.508 \\
30000.0 & 9.508 \\
30500.0 & 9.508 \\
31000.0 & 9.508 \\
31500.0 & 9.508 \\
32000.0 & 9.508 \\
32500.0 & 9.508 \\
33000.0 & 9.508 \\
33500.0 & 9.508 \\
34000.0 & 9.508 \\
34500.0 & 9.508 \\
35000.0 & 9.508 \\
35500.0 & 9.508 \\
36000.0 & 9.508 \\
36500.0 & 9.508 \\
37000.0 & 9.508 \\
37500.0 & 9.508 \\
38000.0 & 9.508 \\
38500.0 & 9.508 \\
39000.0 & 9.508 \\
39500.0 & 9.508 \\
40000.0 & 9.508 \\
40500.0 & 9.508 \\
41000.0 & 9.508 \\
41500.0 & 9.508 \\
$E N D$ & \\
\hline &
\end{tabular}




\section{HSB $69 A$ \\ MAY 9, 1990 \\ TEST TWO \\ RECOVERY DATA}

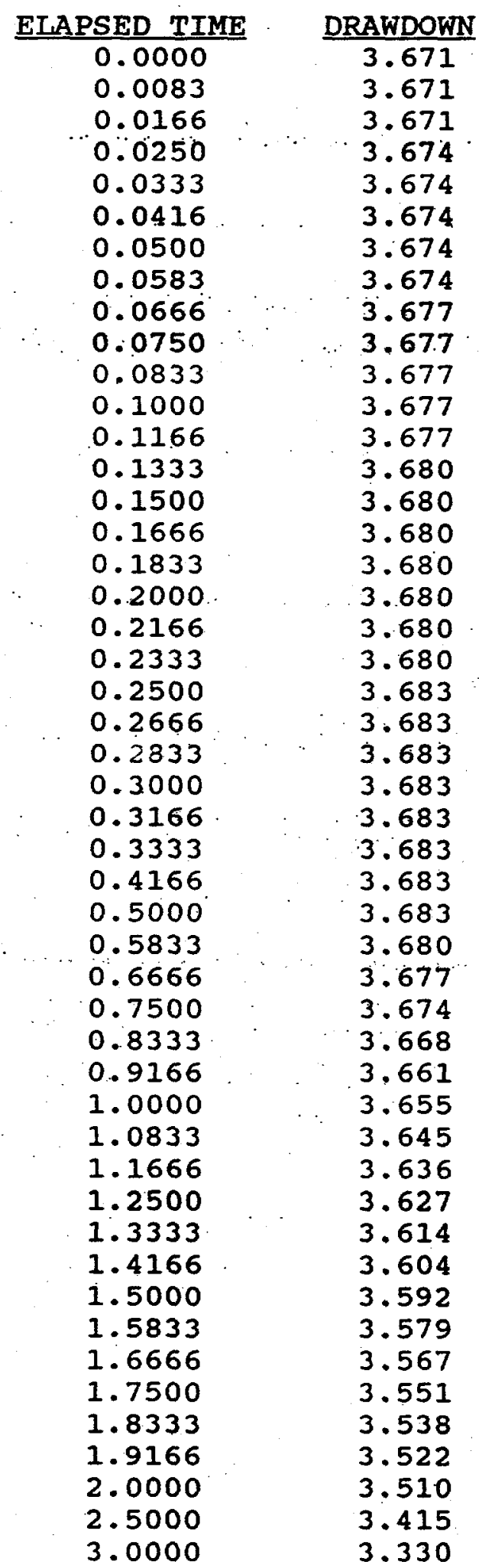




\begin{tabular}{|c|c|c|}
\hline $\begin{array}{l}3.5000 \\
4.0000 \\
4.5000 \\
5.0000 \\
5.5000 \\
6.0000 \\
6.5000 \\
7.0000 \\
7.5000 \\
8.0000 \\
8.5000 \\
9.0000 \\
9.5000 \\
0.0000 \\
2.0000 \\
4.0000 \\
6.0000 \\
8.0000 \\
0.0000 \\
2.0000 \\
4.0000 \\
6.0000 \\
8.0000 \\
0.0000 \\
2.0000 \\
4.0000 \\
6.0000 \\
8.0000 \\
0.0000 \\
2.0000 \\
4.0000 \\
6.0000 \\
8.0000 \\
0.0000 \\
2.0000 \\
4.0000 \\
6.0000 \\
8.0000 \\
0.0000 \\
2.0000 \\
4.0000 \\
6.0000 \\
8.0000 \\
0.0000 \\
2.0000 \\
4.0000 \\
6.0000 \\
8.0000 \\
0.0000 \\
2.0000 \\
4.0000 \\
6.0000 \\
8.0000 \\
0.0000\end{array}$ & $\cdot \cdots$ & $\begin{array}{l}3.248 \\
3.169 \\
3.099 \\
3.033 \\
2.976 \\
2.919 \\
2.869 \\
2.822 \\
2.781 \\
2.739 \\
2.702 \\
2.664 \\
2.632 \\
2.601 \\
2.477 \\
2.376 \\
2.298 \\
2.225 \\
2.165 \\
2.108 \\
2.058 \\
2.010 \\
1.966 \\
1.925 \\
1.887 \\
1.852 \\
1.818 \\
1.786 \\
1.758 \\
1.729 \\
1.704 \\
1.679 \\
1.654 \\
1.631 \\
1.609 \\
1.587 \\
1.565 \\
1.549 \\
1.530 \\
1.512 \\
1.493 \\
1.3325 \\
1.4773 \\
1.461 \\
1.445 \\
1.433 \\
1.417 \\
1.401 \\
1.385 \\
1.373\end{array}$ \\
\hline
\end{tabular}




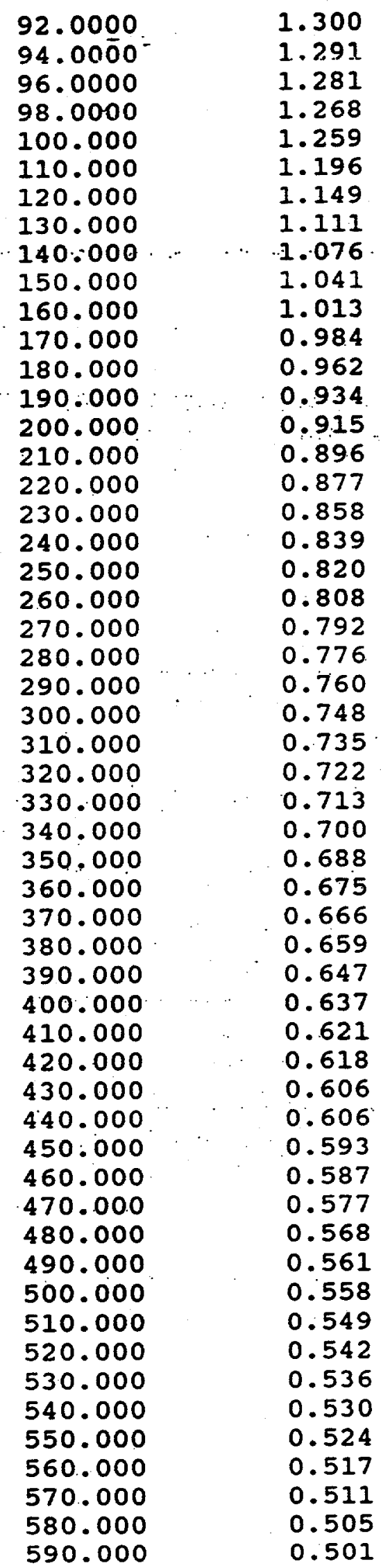




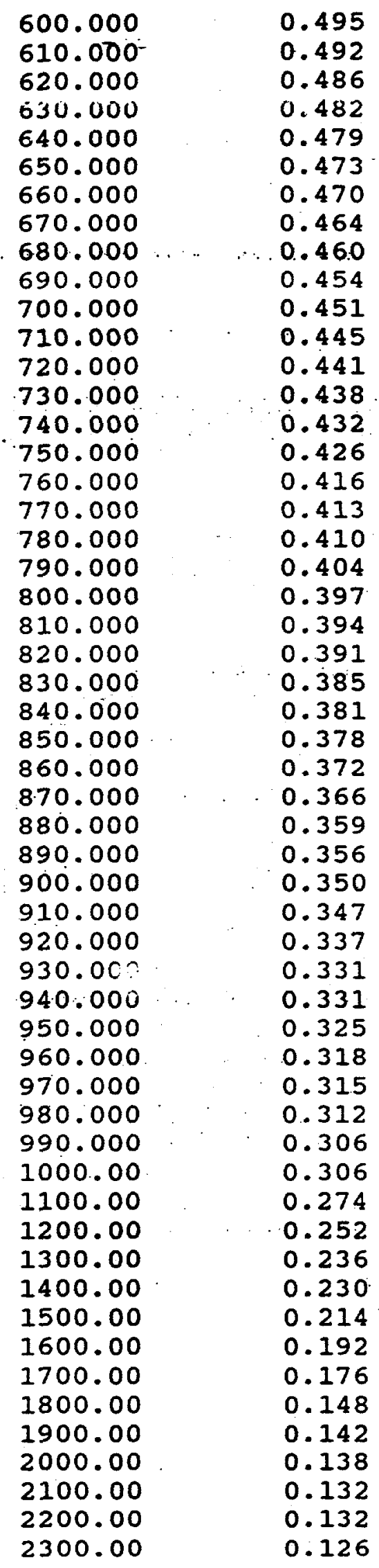




$\begin{array}{ll}2400.00 & 0.116 \\ 2500.00 & 0.110 \\ 2600.00 & 0.101 \\ 2700.00 & 0.097 \\ 2800.00 & 0.104 \\ 2900.00 & 0.104 \\ 3000.00 & 0.101 \\ 3100.00 & 0.091 \\ 3200.00 & 0.088 \\ 3300.00 & 0.075 \\ 3400.00 & 0.063 \\ 3500.00 & 0.056 \\ 3600.00 & 0.059 \\ 3700.00 & 0.063 \\ 3800.00 & 0.059 \\ 3900.00 & 0.056 \\ 4000.00 & 0.053 \\ 4100.00 & 0.050 \\ 4200.00 & 0.050 \\ 4300.00 & 0.059 \\ 4400.00 & 0.059 \\ 4500.00 & 0.056 \\ 4600.00 & 0.047 \\ 4700.00 & 0.034 \\ 4800.00 & 0.015 \\ 4900.00 & 0.012 \\ 5000.00 & 0.009 \\ 5100.00 & 0.006 \\ 5200.00 & 0.003 \\ 5300.00 & 0.000 \\ 5400.00 & -0.003 \\ 5500.00 & -0.025 \\ 5600.00 & -0.031 \\ 5700.00 & -0.037 \\ 5800.00 & -0.031 \\ 5900.00 & -0.028 \\ 6000.00 & -0.034 \\ 6100.00 & -0.041 \\ 6200.00 & -0.047 \\ 6300.00 & -0.050 \\ 6400.00 & -0.050 \\ 6500.00 & -0.050 \\ 6600.00 & -0.050 \\ 6700.00 & -0.053 \\ 6800.00 & -0.059 \\ 6900.00 & -0.063 \\ 7000.00 & -0.063 \\ 7100.00 & -0.053 \\ 7200.00 & -0.053 \\ 7300.00 & -0.050 \\ 7400.00 & \\ 7500.00 & \\ 7600.00 & -0.034 \\ 7700.00 & \end{array}$




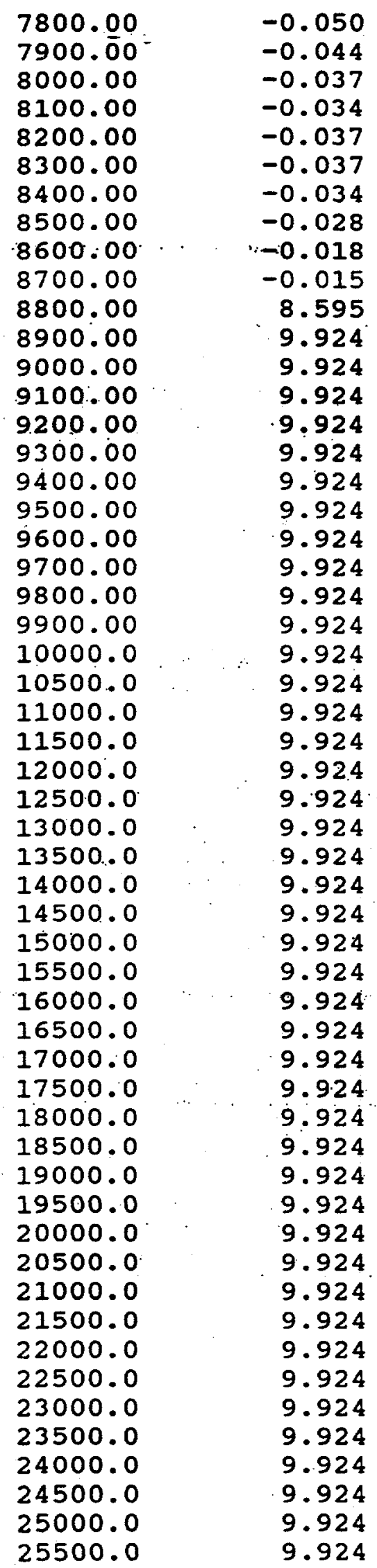




\begin{tabular}{ll}
26000.0 & 9.924 \\
26500.0 & 9.924 \\
27000.0 & 9.924 \\
27500.0 & 9.924 \\
28000.0 & 9.924 \\
28500.0 & 9.924 \\
29000.0 & 9.924 \\
29500.0 & 9.924 \\
30000.0 & 9.924 \\
30500.0 & 9.924 \\
31000.0 & 9.924 \\
31500.0 & 9.924 \\
32000.0 & 9.924 \\
32500.0 & 9.924 \\
33000.0 & 9.924 \\
33500.0 & 9.924 \\
34000.0 & 9.924 \\
34500.0 & 9.924 \\
35000.0 & 9.924 \\
35500.0 & 9.924 \\
36000.0 & 9.924 \\
36500.0 & 9.924 \\
37000.0 & 9.924 \\
37500.0 & 9.924 \\
38000.0 & 9.924 \\
38500.0 & 9.924 \\
39000.0 & 9.924 \\
39500.0 & 9.924 \\
40000.0 & 9.924 \\
40500.0 & 9.924 \\
41000.0 & 9.924 \\
41500.0 & 9.924 \\
\hline 10 &
\end{tabular}


WELL NO.: $86 \mathrm{~A}$

PAGE 1 OF 2

DISTANCE FROM PUMPING WELL: $996 \mathrm{FT}$

DATE: May 8, 1990

\begin{tabular}{|l|c|c|c|c|c|c|}
\hline TIME & $\begin{array}{c}\text { ELAPSED } \\
\text { TIME (MIN) }\end{array}$ & $\begin{array}{c}\text { DEPTH TO } \\
\text { WATER (TOC) }\end{array}$ & DRAWDOWN & $\begin{array}{c}\text { FLOW- } \\
\text { METER } \\
\text { READING }\end{array}$ & Q (GPM) & MBAR \\
\hline $12: 30$ & 0 & 95.70 & 0 & & & \\
\hline $13: 24$ & 54 & 95.82 & 0.12 & & & \\
\hline $13: 51$ & 81 & 95.91 & 0.21 & & & \\
\hline $14: 15$ & 105 & 95.96 & 0.21 & & & \\
\hline $15: 00$ & 150 & 96.06 & 0.36 & & & \\
\hline $15: 28$ & 178 & 96.11 & 0.41 & & & \\
\hline $16: 42$ & 252 & 96.22 & 0.52 & & & \\
\hline $17: 33$ & 303 & 96.30 & 0.60 & & & \\
\hline $18: 38$ & 368 & 96.38 & 0.68 & & & \\
\hline $19: 30$ & 420 & 96.43 & 0.73 & & & \\
\hline $20: 16$ & 466 & 96.45 & 0.75 & & & \\
\hline $21: 14$ & 524 & 96.48 & 0.78 & & & \\
\hline $22: 25$ & 595 & 96.53 & 0.83 & & & \\
\hline $23: 26$ & 656 & 96.57 & 0.87 & & & \\
\hline $00: 23$ & 713 & 96.59 & 0.89 & & & \\
\hline $02: 14$ & 824 & 96.64 & 0.94 & & & \\
\hline $24: 04$ & 934 & 96.68 & 0.98 & & & \\
\hline $66: 06$ & 1056 & 96.72 & 1.02 & & & \\
\hline $08: 02$ & 1170 & 96.78 & 1.06 & & & \\
\hline $69: 04$ & 1232 & 96.85 & 1.13 & & & \\
\hline & & & & & & \\
\hline & & Pump turned off at & $10: n n$ A. M. & & & \\
\hline
\end{tabular}


WELL NO.: HSB 86A

PAGE 2 OF 2

DISTANCE FROM PUMPING WELL: $996 \mathrm{FT}$

DATE: May 9, 1990

\begin{tabular}{|c|c|c|c|c|c|c|}
\hline TIME & $\begin{array}{l}\text { ELAPSED } \\
\text { TIME (MIN) }\end{array}$ & $\begin{array}{l}\text { DEPTH TO } \\
\text { WATER (TOC) }\end{array}$ & DRAWDOWN & $\begin{array}{l}\text { FLOW- } \\
\text { METER } \\
\text { READING }\end{array}$ & $Q(G P M)$ & MBAR \\
\hline $10: 00$ & 0 & 2 & & & & \\
\hline $10: 37$ & 37 & 96.80 & 1.10 & & & \\
\hline $10: 58$ & 58 & 96.74 & 1.04 & & $\therefore$ & \\
\hline $11: 20$ & 80 & 96.70 & 1.0 & & & \\
\hline $11: 45$ & 105 & 96.65 & 0.95 & & & \\
\hline $12: 55$ & 175 & 96.46. & 0.76 & & & \\
\hline $13: 17$ & 197 & 96.44 & 0.74 & & & \\
\hline 13.39 & 219 & 96.44 & & & & $\therefore$ \\
\hline $14: 33$ & 273 & 96.37 & 0.67 & & & \\
\hline 15.33 & 333 & 96.31 & 0.61. & & & \\
\hline $16: 35$ & 395 & 96.24 & 0.54 & & & \\
\hline $17: 36$ & 456 & 96.21 & 0.51 & & & \\
\hline $18: 36$ & 516 & 96.17 & 0.47 & & & \\
\hline $19: 31$ & $571^{\circ}$ & 96.15 & 0.45 & & & \\
\hline $23: 57$ & 837 & 96.03 & 0.33 & & 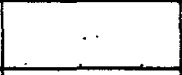 & \\
\hline $04: 05$ & 1085 & 95.94 & 0.24 & & - & \\
\hline $07: 48$ & 1308 & 95.90 & 0.20 & & & \\
\hline $09: 53$ & 1433 & 95.88 & 0.18 & & & \\
\hline & & & & & & \\
\hline & & & & & & \\
\hline & & & & & & \\
\hline
\end{tabular}




\section{$\sum^{\prime \prime} i^{2}$}

WELL NO.: HSB 118A

FAGE I OF 3

DISTANCE FROM PUMPING WELL: $1244 \mathrm{Ft}$

DATE: May 8, 1990

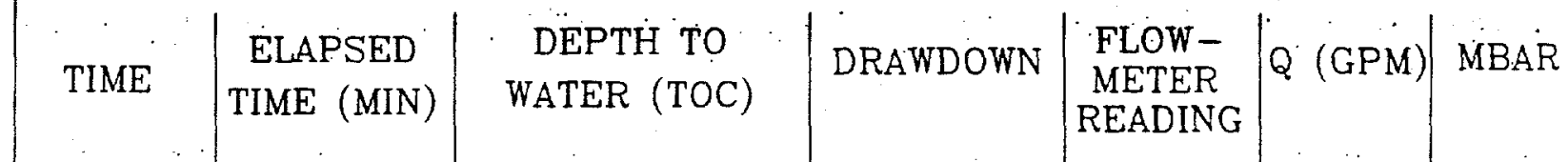

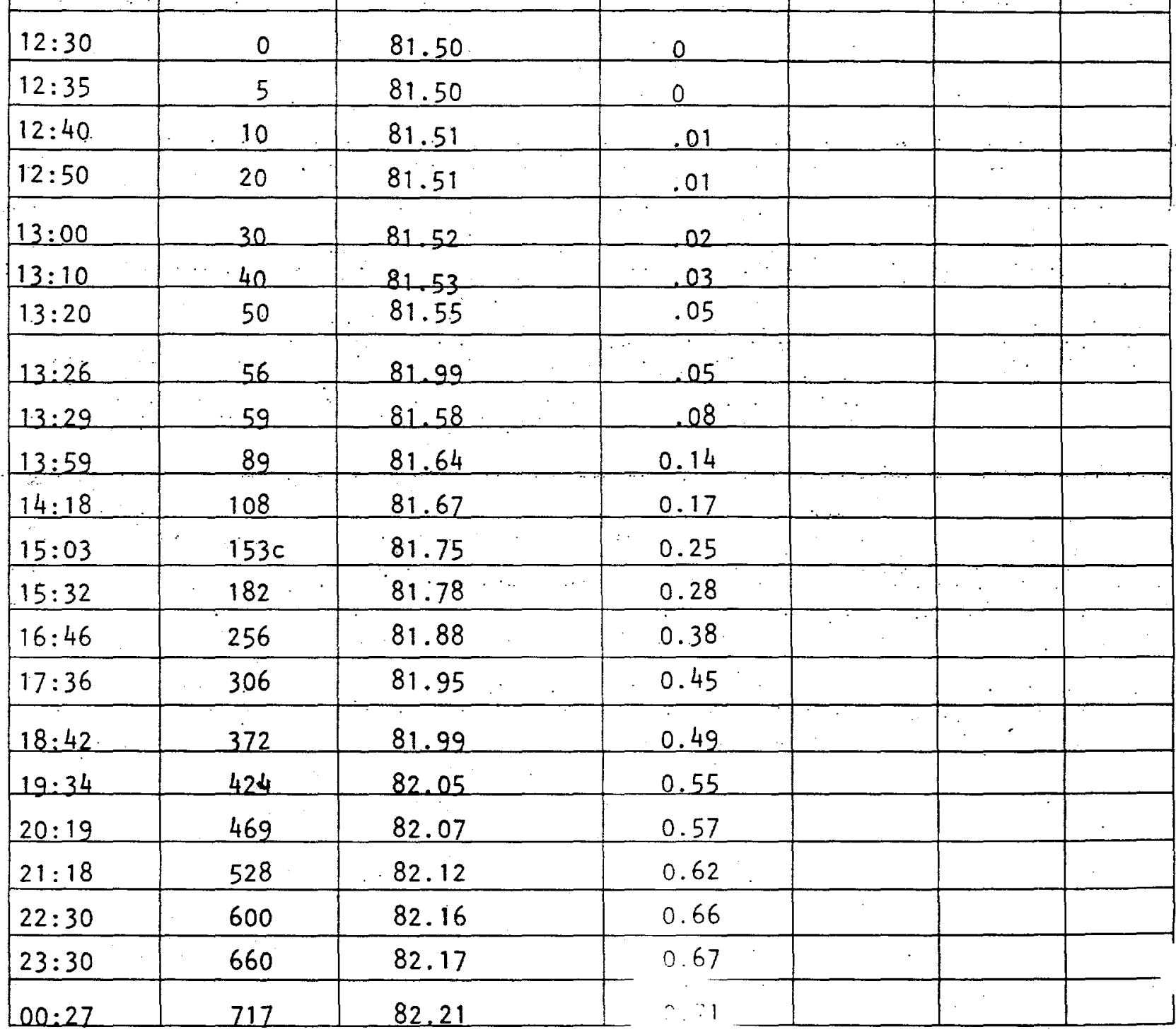


WELL NO.: HSB 118A

PAGE 2 OF 3

DISTANCE FROM PUMPING WELL: 1244 FT

DATE: May 9, 1990

TIME

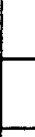

\begin{tabular}{|c|c|c|c|} 
& TIME (MIN) & WATER (TOC) & DRAWDOWN \\
\hline $02: 18$ & 828 & 82.26 & 0.76 \\
\hline
\end{tabular}

FLOW-

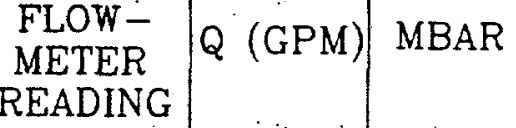

\begin{tabular}{|c|c|c|c|}
\hline $04: 08$ & 938 & 82.29 & 0.79 \\
\hline $06: 09$ & 1059 & 82.32 & 0.82 \\
\hline $08: 06$ & 1176 & 82.38 & 0.88 \\
\hline $09: 08$ & 1238 & 82.40 & 0.90 \\
\hline & & Stopped pumping a & $10: 00 \mathrm{~A} . \mathrm{M}$ \\
\hline & & REÇOVERY & \\
\hline
\end{tabular}

\begin{tabular}{|l|c|c|c|l|l|l|}
\hline $10: 00$ & 0 & $?$ & $?$ & & & \\
\hline $10: 39$ & 39 & 82.40 & 0.9 & & & \\
\hline $11: 00$ & 60 & 82.38 & 0.88 & & & \\
\hline 11.23 & 83 & 82.34 & 0.84 & & & \\
\hline $11: 48$ & 108 & 82.31 & 0.81 & & & \\
\hline $11: 58$ & $118(178)$ & 82.20 & 0.70 & & & \\
\hline $13: 20$ & 200 & 82.18 & 0.68 & & & \\
\hline $13: 41$ & 221 & 82.18 & 0.68 & & & \\
\hline $14: 36$ & 276 & 82.11 & 0.61 & & & \\
\hline $15: 36$ & 336 & 82.05 & 0.55 & & & \\
\hline $16: 38$ & 398 & 82.01 & 0.51 & & & \\
\hline $17: 39$ & 459 & 81.98 & 0.48 & & & \\
\hline $18: 39$ & 519 & 81.95 & 0.45 & & & \\
\hline $19: 33$ & 573 & 81.93 & 0.43 & & & \\
\hline $00: 01$ & 841 & 81.83 & 0.33 & & & \\
\hline
\end{tabular}




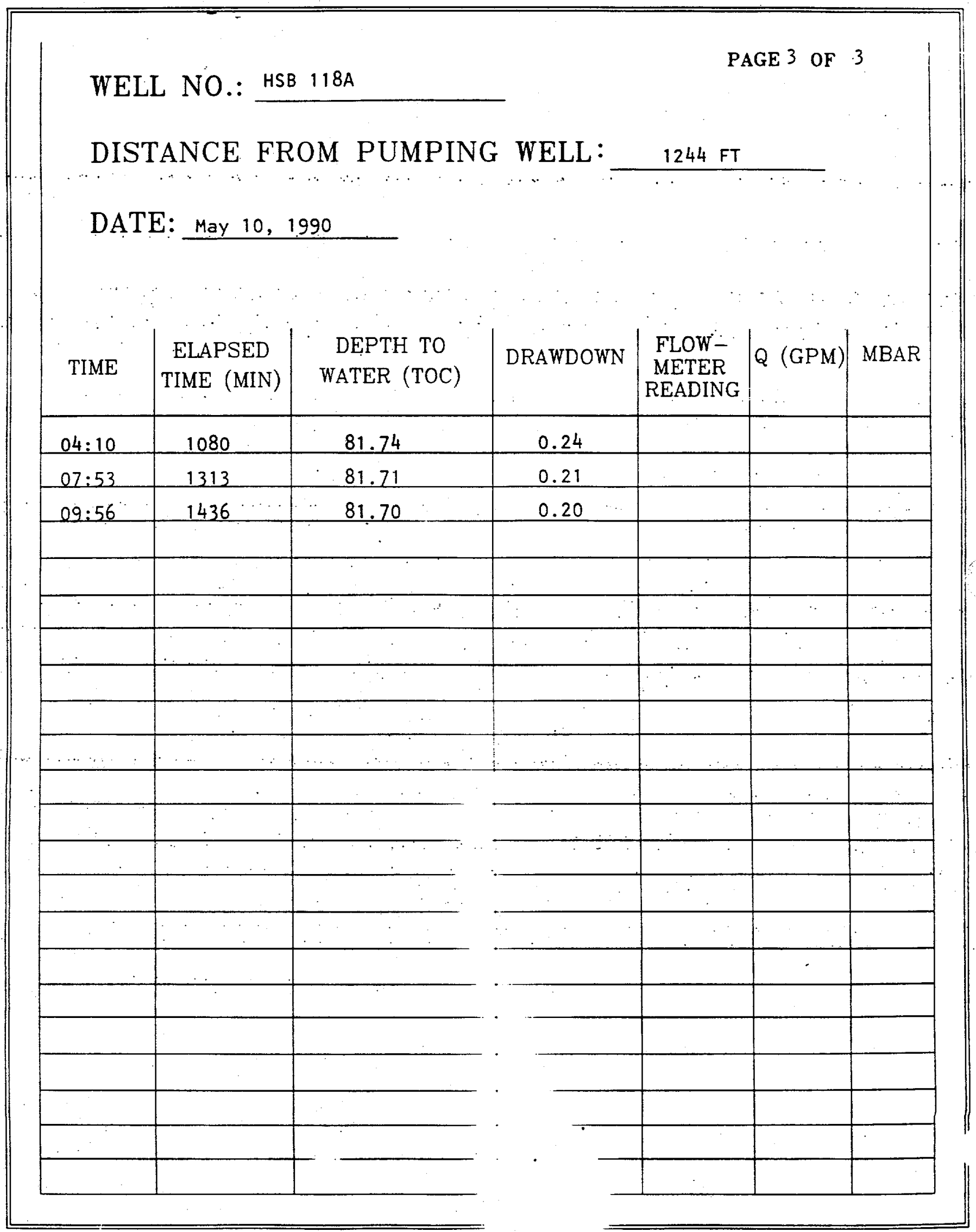


WELL NO.: HSB 68A

PAGE 1 OF 4

DISTANCE FROM PUMPING WELL: $549 \mathrm{FT}$

DATE: May 8,1990

\begin{tabular}{|c|c|c|c|c|c|c|}
\hline TIME & $\begin{array}{c}\text { ELAPSED } \\
\text { TIME (MIN) }\end{array}$ & $\begin{array}{c}\text { DEPTH TO } \\
\text { WATER (TOC) }\end{array}$ & DRAWDOWN & $\begin{array}{c}\text { FLOW- } \\
\text { MEERR }\end{array}$ & Q (GPM) & MBAR \\
\hline $12: 30$ & 0 & 79.56 & 0 & & & \\
\hline $12: 31$ & 1 & 79.56 & 0 & & & \\
\hline $12: 32$ & 2 & 79.58 & 0.02 & & & \\
\hline $12: 33$ & 3 & 79.59 & 0.03 & & & \\
\hline $12: 34$ & 4 & 79.59 & 0.03 & & & \\
\hline $12: 35$ & 5 & 79.60 & 0.04 & & & \\
\hline $12: 40$ & 10 & 79.65 & 0.09 & & & \\
\hline $12: 45$ & 15 & 79.73 & 0.17 & & & \\
\hline $12: 50$ & 20 & 79.80 & 0.24 & & & \\
\hline $12: 55$ & 25 & 79.87 & 0.31 & & & \\
\hline $13: 00$ & 30 & 79.92 & 0.36 & & & \\
\hline $13: 05$ & 35 & 79.95 & 0.39 & & & \\
\hline $13: 10$ & 40 & 79.99 & 0.43 & & & \\
\hline $13: 20$ & 50 & 80.06 & 0.50 & & & \\
\hline $13: 33$ & 63 & 80.12 & 0.56 & & & \\
\hline $14: 02$ & 92 & 80.25 & 0.69 & & & \\
\hline $14: 22$ & 112 & 80.32 & 0.76 & & & \\
\hline $15: 08$ & 158 & 80.46 & 0.90 & & & \\
\hline $15: 36$ & 186 & 80.51 & 0.95 & & & \\
\hline $16: 52$ & 262 & 80.66 & 1.10 & & & \\
\hline 17.42 & 312 & 80.74 & 1.18 & & & \\
\hline $18: 49$ & 379 & 80.82 & 1.26 & & & \\
\hline
\end{tabular}


WELL NO.: HSB 68A

PAGE 2 OF 4

DISTANCE FROM PUMPING WELL: $545 \mathrm{FT}$

DATE: May 8, 1990

MAY 9

\begin{tabular}{|c|c|c|c|c|c|c|}
\hline TIME & $\begin{array}{l}\text { ELAPSED } \\
\text { TIME (MIN) }\end{array}$ & $\begin{array}{c}\text { DEPTH TO } \\
\text { WATER (TOC) } \\
80.82 \\
\end{array}$ & DRAWDOWN & $\begin{array}{c}\text { FLOW- } \\
\text { METER } \\
\text { READING }\end{array}$ & $Q(G P M)$ & MBAR \\
\hline $19: 38$ & 428 & 80.85 & 1.29 & & & \\
\hline $20: 23$ & 473 & 80.88 & 1.32 & & & \\
\hline $21: 24$ & 534 & 80.89 & 1.33 & & & \\
\hline $22: 36$ & 606 & 80.97 & 1.41 & & & \\
\hline $23: 35$ & 665 & 81.0 & 1.44 & & & \\
\hline $00: 32$ & 722 & 81.04 & 1.48 & & & \\
\hline 02.23 & .833 & 81.11 & 1.55 & & & \\
\hline $04: 13$ & 243 & 81.13 & 1.57 & & & \\
\hline $06: 13$ & 1063 & 81.18 & 1.62 & & & \\
\hline $08: 08$ & 1178 & 81.29 & 1.73 & & & \\
\hline \multirow[t]{3}{*}{$09: 13$} & 1243 & 8.1 .31 & 1.75 & & & \\
\hline & & Stopped pumping & $10: 00$ A. M. & & & \\
\hline & & RECOVERY & & - & & \\
\hline \multirow[t]{2}{*}{$10: 00$} & 0. & $?$ & $?$ & - & & \\
\hline & & & & . & & \\
\hline & & & & & : & \\
\hline & & & & & & \\
\hline & & & & & & \\
\hline & & & & & & \\
\hline & & & & & & \\
\hline & & & & & & \\
\hline & & & & & & \\
\hline
\end{tabular}




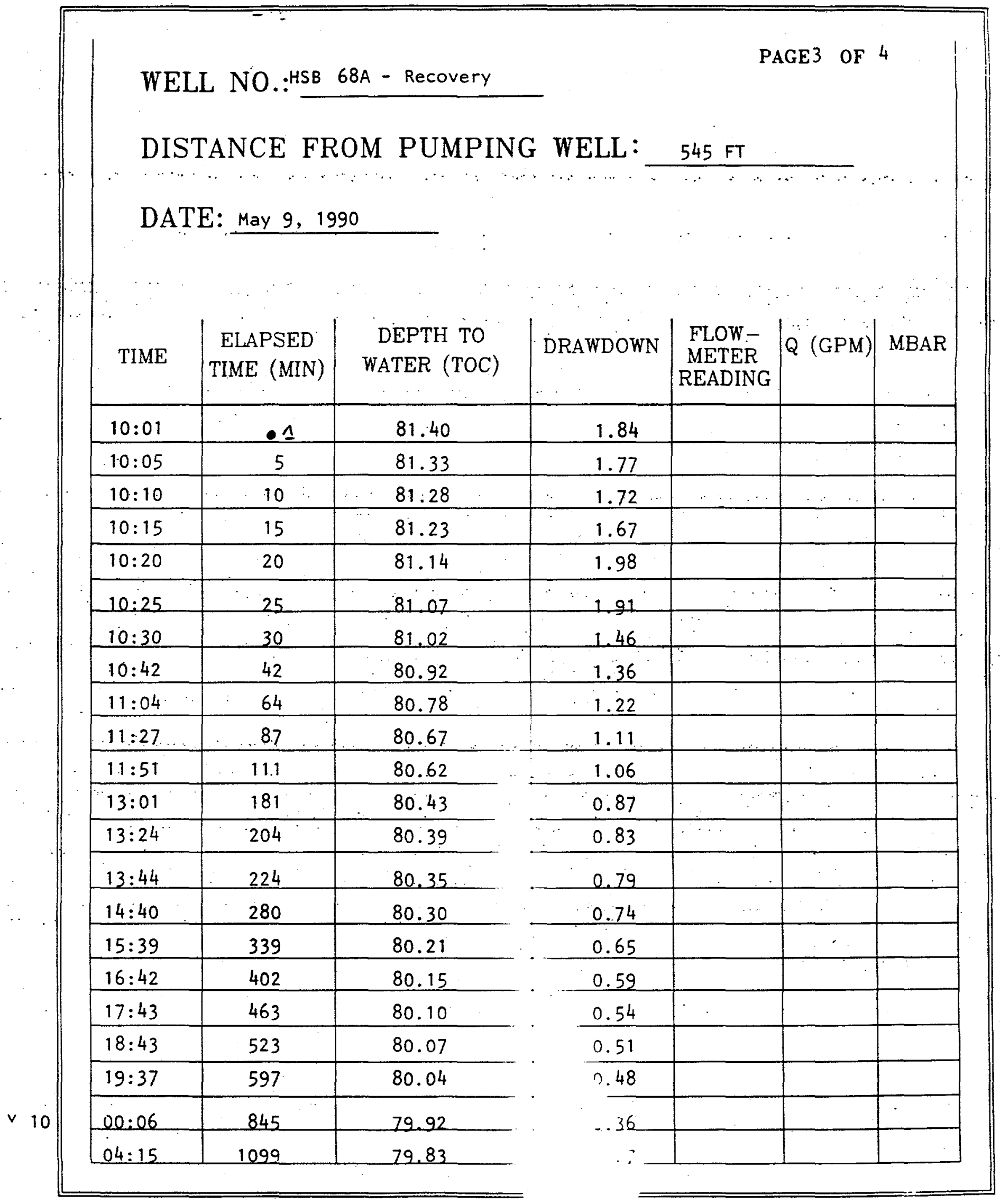




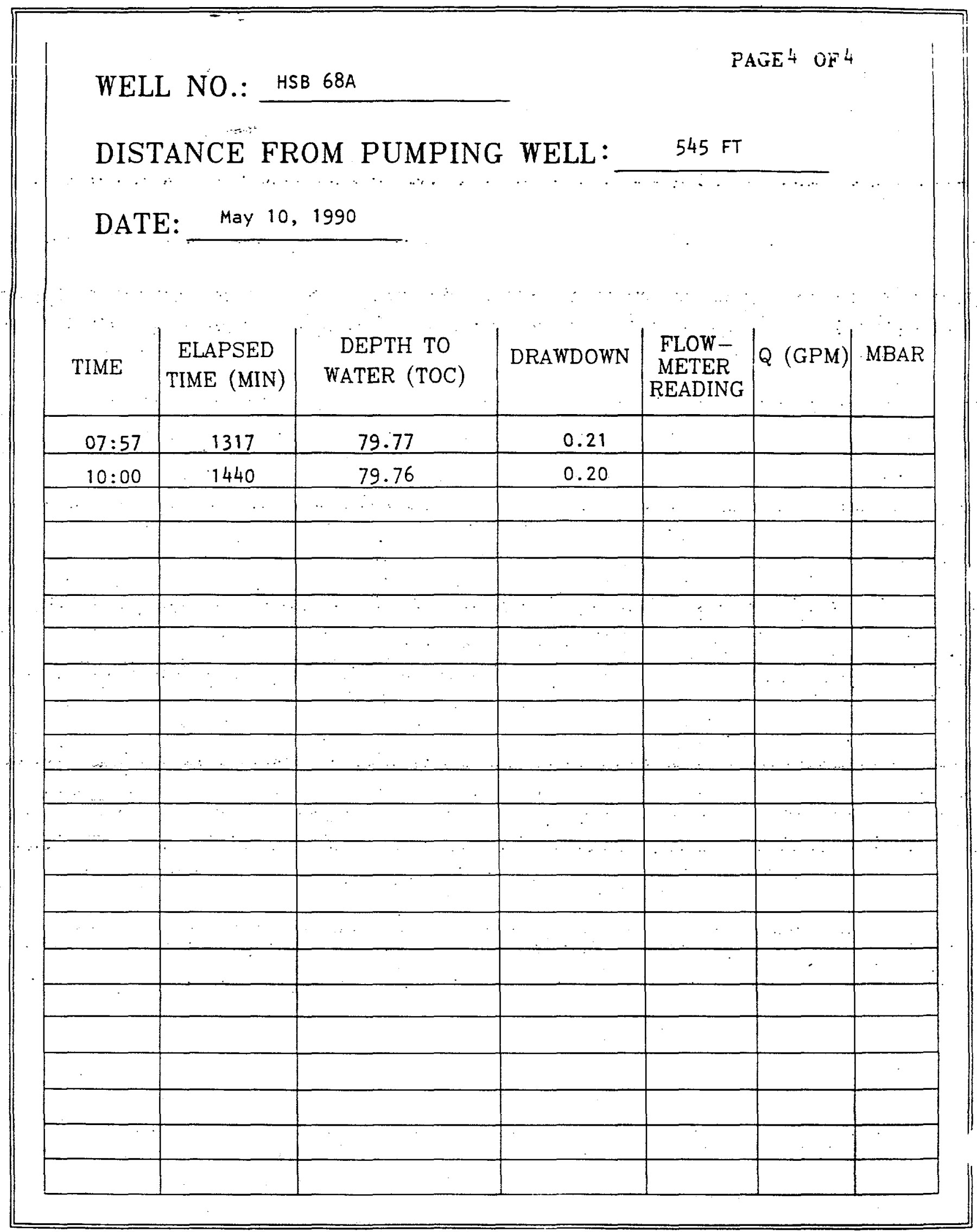


WELL NO.: HSB 139A

PAGE 1 OF 2

DISTANCE FROM PUMPING WELL: 1116 FT

DATE: May 8, 1990

\begin{tabular}{|l|c|c|c|c|c|c|}
\hline TIME & $\begin{array}{c}\text { ELAPSED } \\
\text { TIME (MIN) }\end{array}$ & $\begin{array}{c}\text { DEPTH TO } \\
\text { WATER (TOC) }\end{array}$ & DRAWDOWN & $\begin{array}{c}\text { FLOW- } \\
\text { METER } \\
\text { READING }\end{array}$ & Q (GPM) & MBAR \\
\hline $12: 30$ & 0 & 62.12 & 0 & & & \\
\hline $13: 37$ & 67 & 62.22 & 0.10 & & & \\
\hline $14: 05$ & 95 & 62.28 & 0.16 & & & \\
\hline $14: 25$ & 115 & 62.32 & 0.20 & & & \\
\hline $15: 12$ & 162 & 62.42 & 0.30 & & & \\
\hline $15: 39$ & 189 & 62.47 & 0.35 & & & \\
\hline $16: 56$ & 266 & 62.60 & 0.48 & & & \\
\hline $17: 44$ & 314 & 62.66 & 0.54 & & & \\
\hline $18: 51$ & 381 & 62.76 & 0.64 & & & \\
\hline $19: 43$ & 433 & 62.81 & 0.69 & & & \\
\hline $20: 27$ & 477 & 62.84 & 0.72 & & & \\
\hline $21: 28$ & 538 & 62.88 & 0.76 & & & \\
\hline $22: 41$ & 611 & 62.93 & 0.81 & & $\ddots$ & \\
\hline $23: 39$ & 669 & 62.96 & 0.84 & & & \\
\hline $00: 36$ & 726 & 63.01 & 0.89 & & $\ddots$ & \\
\hline $02: 29$ & 839 & 63.07 & 0.95 & & & \\
\hline $04: 17$ & 947 & 63.11 & 0.99 & & & \\
\hline $06: 17$ & 1067 & 63.16 & 1.05 & & & \\
\hline $08: 11$ & 1181 & 63.24 & 1.13 & & & \\
\hline $09: 17$ & 1247 & 63.25 & 1.14 & & & \\
\hline & & Pumping & & & & \\
\hline & & & & & & \\
\hline & & & & & \\
\hline
\end{tabular}


WELL NO.: HSB 139A

PAGE 2 OF 2

DISTANCE FROM PUMPING WELL: $1116 \mathrm{FT}$

DATE: May 9, 1990

\begin{tabular}{|c|c|c|c|c|c|c|}
\hline TIME & $\begin{array}{c}\text { ELAPSED } \\
\text { TIME (MIN) }\end{array}$ & $\begin{array}{l}\text { DEPTH TO } \\
\text { WATER (TOC) }\end{array}$ & DRAWDOWN & $\begin{array}{l}\text { FLOW- } \\
\text { METER } \\
\text { READING }\end{array}$ & $Q(\mathrm{GPM})$ & MBAR \\
\hline & & RECOVERY & & & & \\
\hline $10: 00$ & 0 & $?$ & $?$ & & & \\
\hline $10: 45$ & 45 & 63.24 & 1.12 & & & \\
\hline $11: 06$ & 66 & 63.20 & 1.08 & & & \\
\hline $11: 30$ & 90 & 63.15 & 1.03 & & & \\
\hline $11: 54$ & 114 & $63.10^{\circ}$ & 0.98 & & & \\
\hline $13: 04$ & 184 & 62.96 & 0.84 & & & \\
\hline $13: 26$ & 206 & 62.95 & 0.83 & & & \\
\hline $13: 47$ & 227 & 62.91 & 0.79 & & & \\
\hline $14: 43$ & 283 & 62.85 & $\cdots 0.73$ & & & \\
\hline $15: 43$ & 343 & 62.79 & 0.67 & & $\cdots$ & \\
\hline $16: 45$ & 405 & 62.72 & 0.60 & & & \\
\hline $17: 46$ & 466 & 62.69 & 0.57 & & & \\
\hline $18: 46$ & 526 & 62.65 & 0.53 & & & \\
\hline $19: 39$. & 579 & 62.61 & 0.49 & & & \\
\hline $00: 11$ & 85.1 & 62.47 & 0.35 & & & \\
\hline $04 \cdot 12$ & 1099 & 62.39 & 0.27 & & & \\
\hline $08: 11$ & 1331 & 62.34 & 0.22 & & & \\
\hline $10: 04$ & 1444 & $62: 3$ & 0.21 & & & \\
\hline & & & & & & \\
\hline & & & & & & \\
\hline & & & & & & \\
\hline
\end{tabular}


WELL NO.: HSB 83A

FAGE I OF 2

DISTANCE FROM PUMPING WELL: 2253 FT

DATE:

May 8,1990

Niay 9

\begin{tabular}{|c|c|c|c|c|c|c|}
\hline TIME & $\begin{array}{l}\text { ELAPSED } \\
\text { TIME (MIN) }\end{array}$ & $\begin{array}{c}\text { DEPTH TO } \\
\text { WATER (TOC) }\end{array}$ & DRAWDOWN & $\begin{array}{c}\text { FLOW- } \\
\text { METER } \\
\text { READING }\end{array}$ & $Q(G P M)$ & MBAR \\
\hline $12: 30$ & 0 & 65.99 & 0 & & & \\
\hline $13: 41$ & 71 & 66.02 & 0.03 & - & & \\
\hline $14: 30$ & 120 & 66.04 & 0.05 & & & \\
\hline $15: 49$ & 199 & 66.10 & 0.11 & & & \\
\hline $17: 00$ & 280 & 66.15 & 0.16 & & . & \\
\hline $17: 49$ & 329 & 66.18 & 0.19 & & & \\
\hline $18: 56$ & 386. & 66.22 & 0.23 & & & \\
\hline $19: 49$ & 439 & 66.25 & 0.26 & & & \\
\hline $21: 35$ & 545 & 66.30 & 0.31 & & & $\because$ \\
\hline $22: 51^{\prime \prime}$ & 621 & $\therefore 66.33$ & 0.34 & & & \\
\hline $23: 44$ & 674 & 66.34 & 0.35 & & & \\
\hline $00: 41$ & 731 & 66.37. & 0.38 & & & \\
\hline $02: 39$ & 849 & 66.42 & 0.42 & & & \\
\hline $04: 22$ & 952 & 66.47 & 0.47 & & & \\
\hline $06: 22$ & 1072 & 66.51 & 0.51 & & & \\
\hline $08: 17$ & 1187 & 66.55 & 0.55 & & & \\
\hline $09: 21$ & 1251 & 66.57 & 0.57 & & & \\
\hline & & PUMFING STOPPED & AT $10.00 \mathrm{~A} .1$ & 1. & & \\
\hline & & RECOVERY & & & & \\
\hline $10: 00$ & 0 & $?$ & $?$ & & & \\
\hline $10: 49$ & 49 & 66.59 & 0.6 & & & \\
\hline $11: 10$ & 70 & 66.59 & 0.6 & & & \\
\hline
\end{tabular}




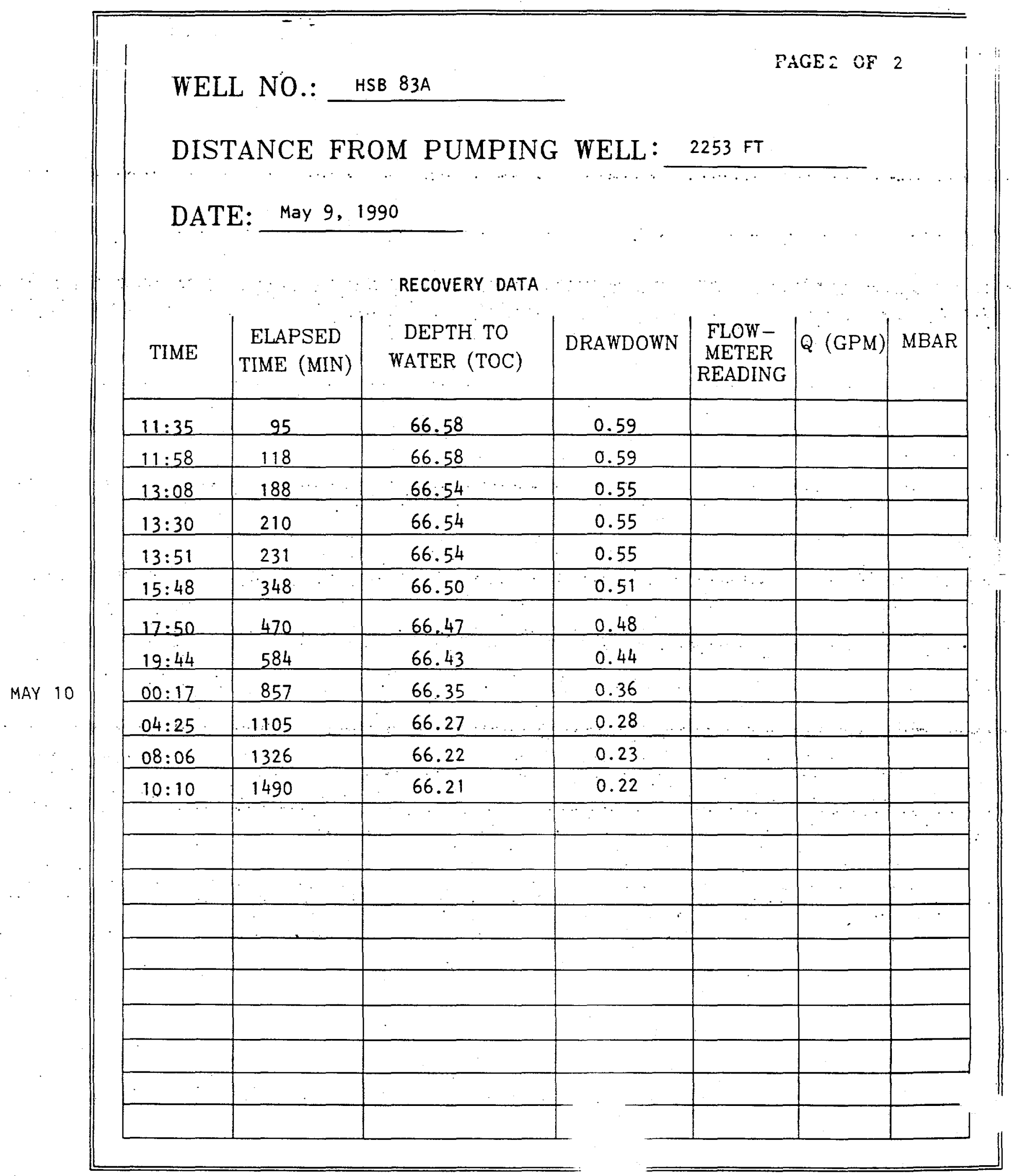


APPENDIX D

AQTESOLV THST TWO 
$\frac{N}{k}$

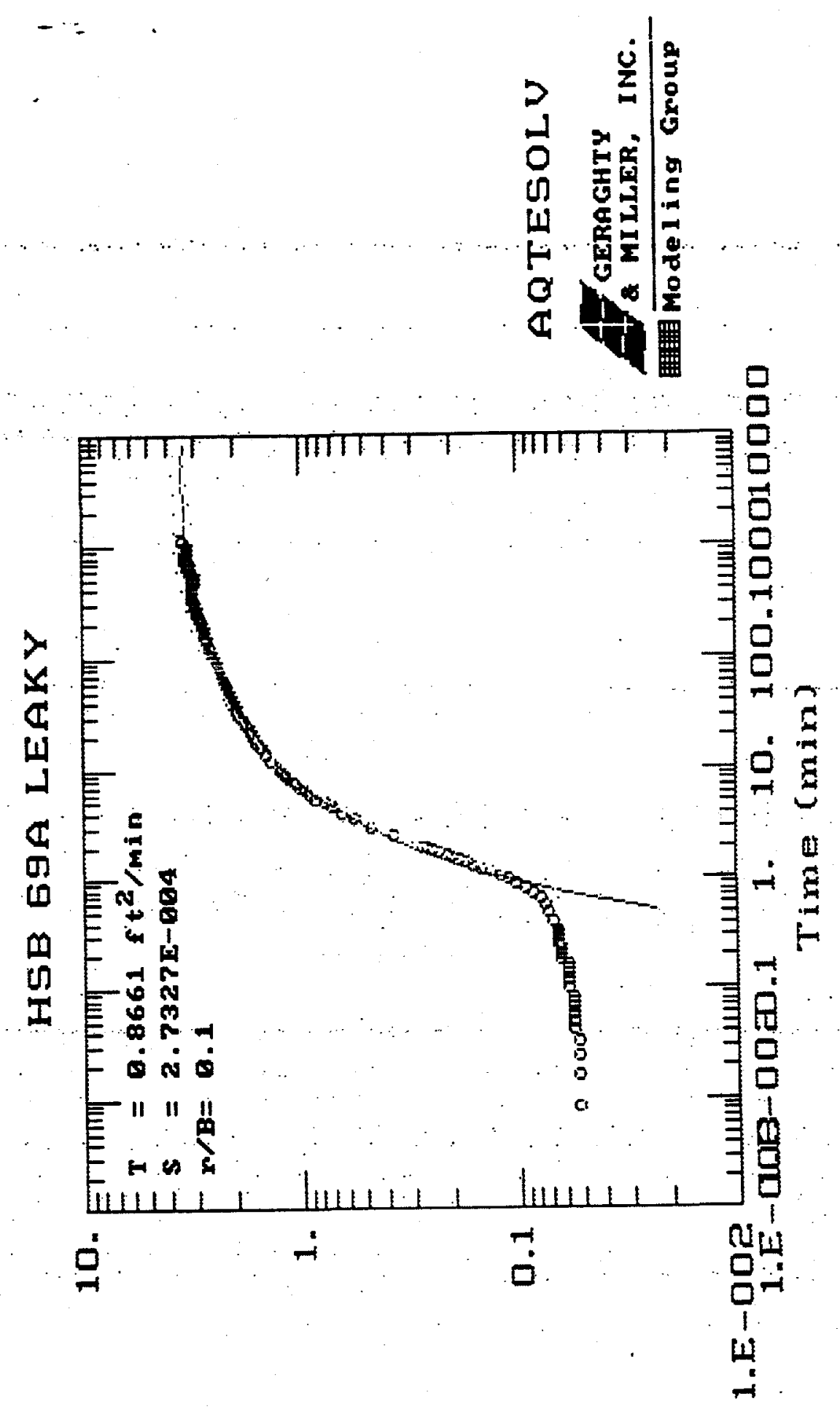

(7) CIMAPMEx 


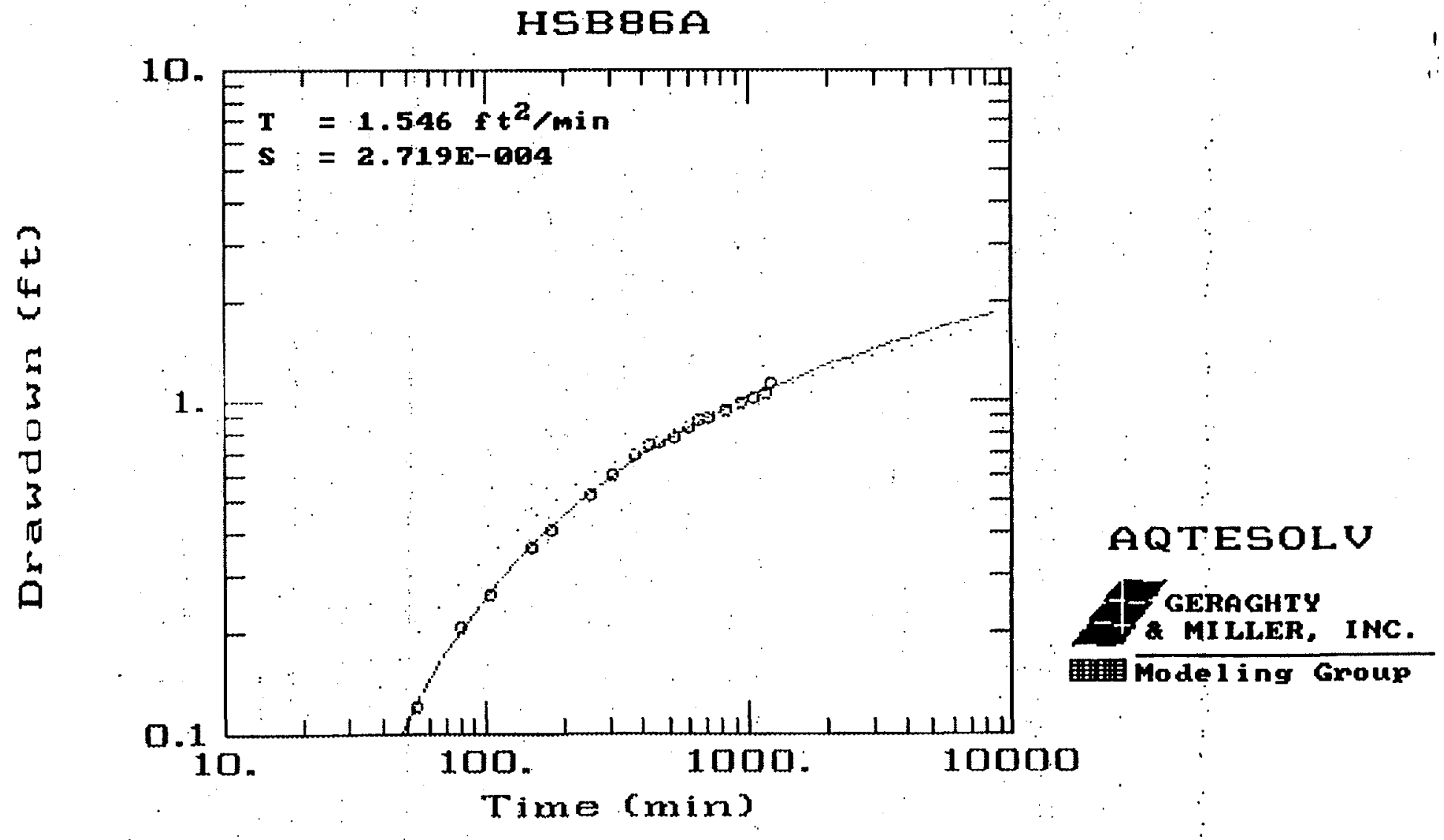




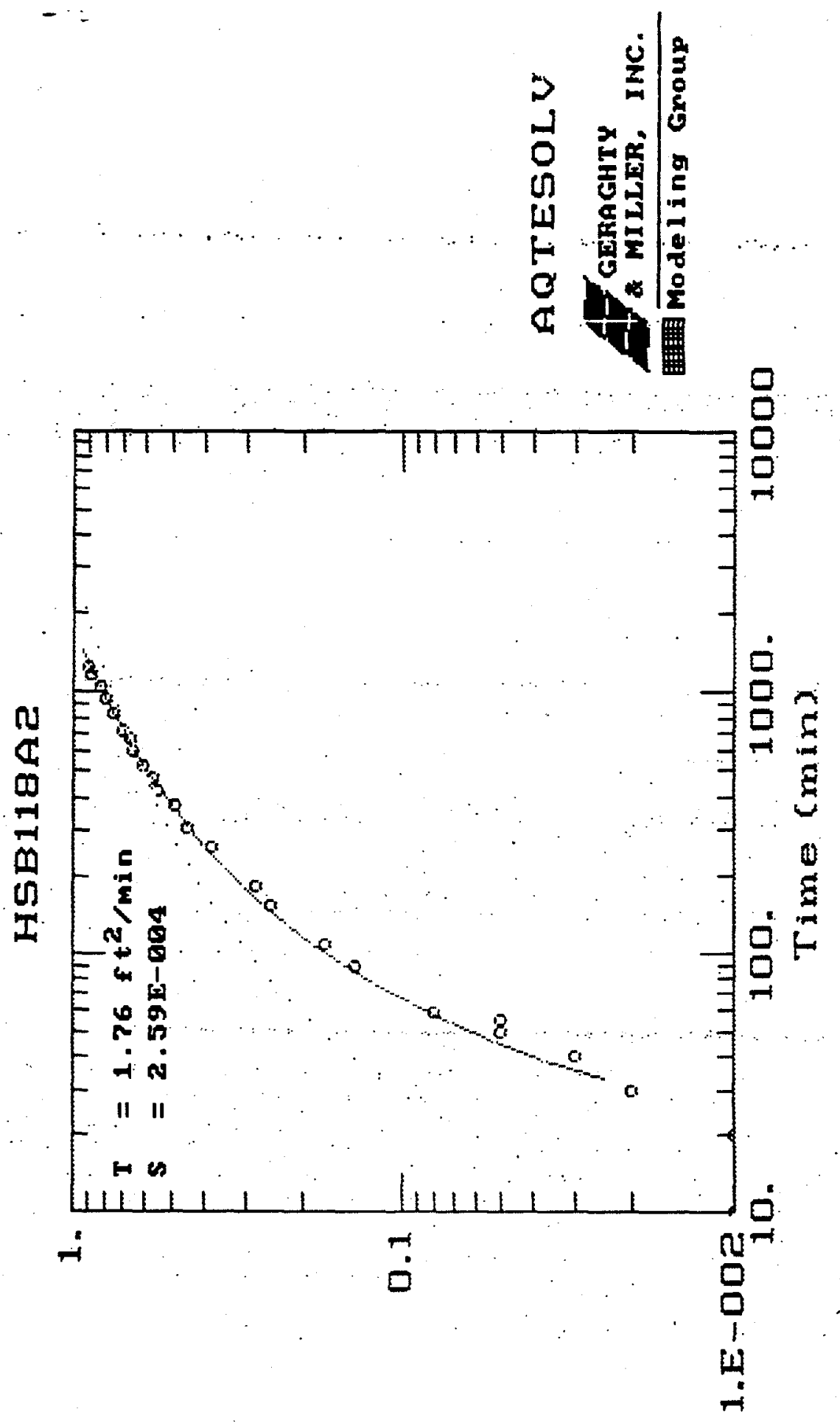

(7) cxMOpmexa 


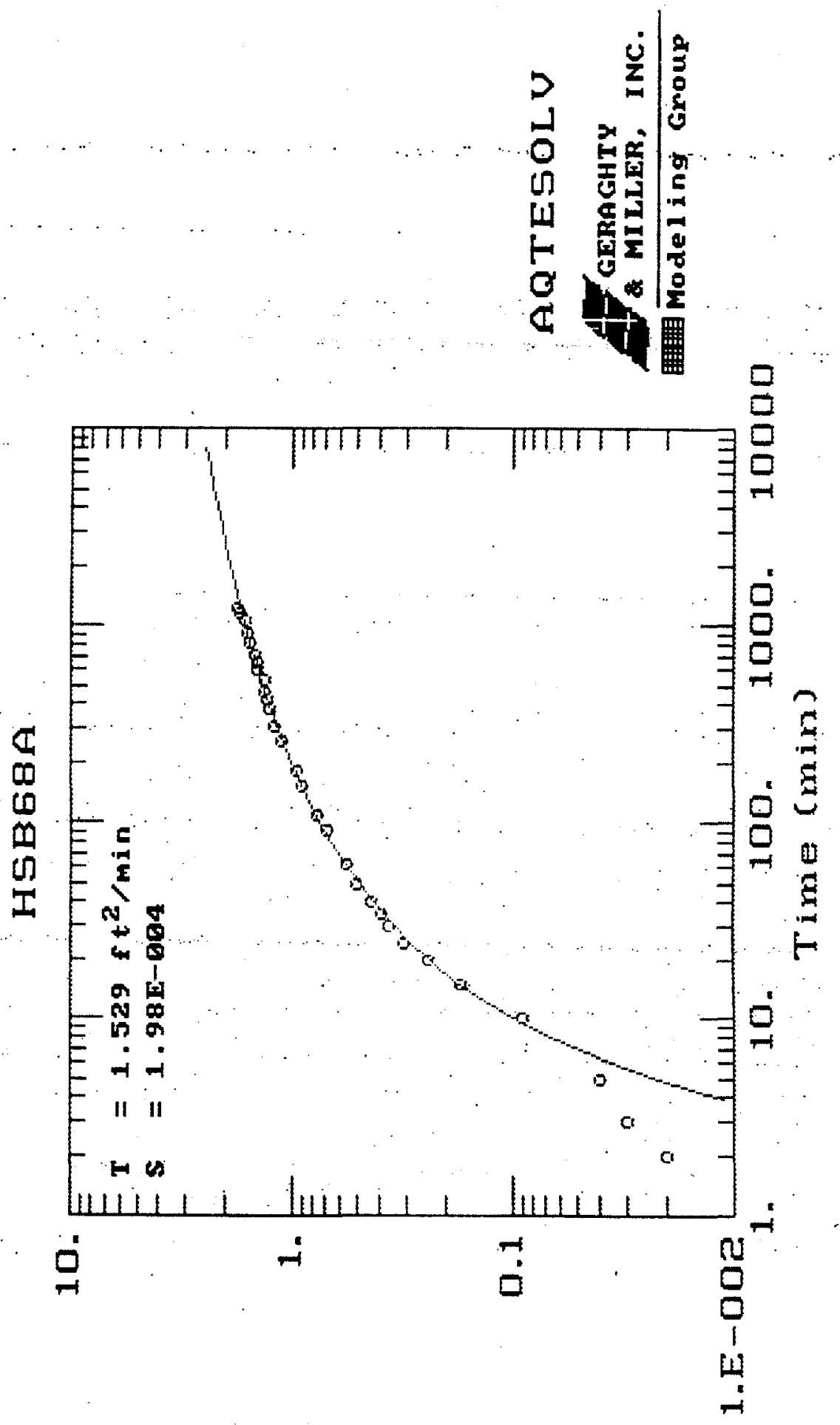

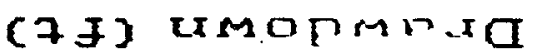




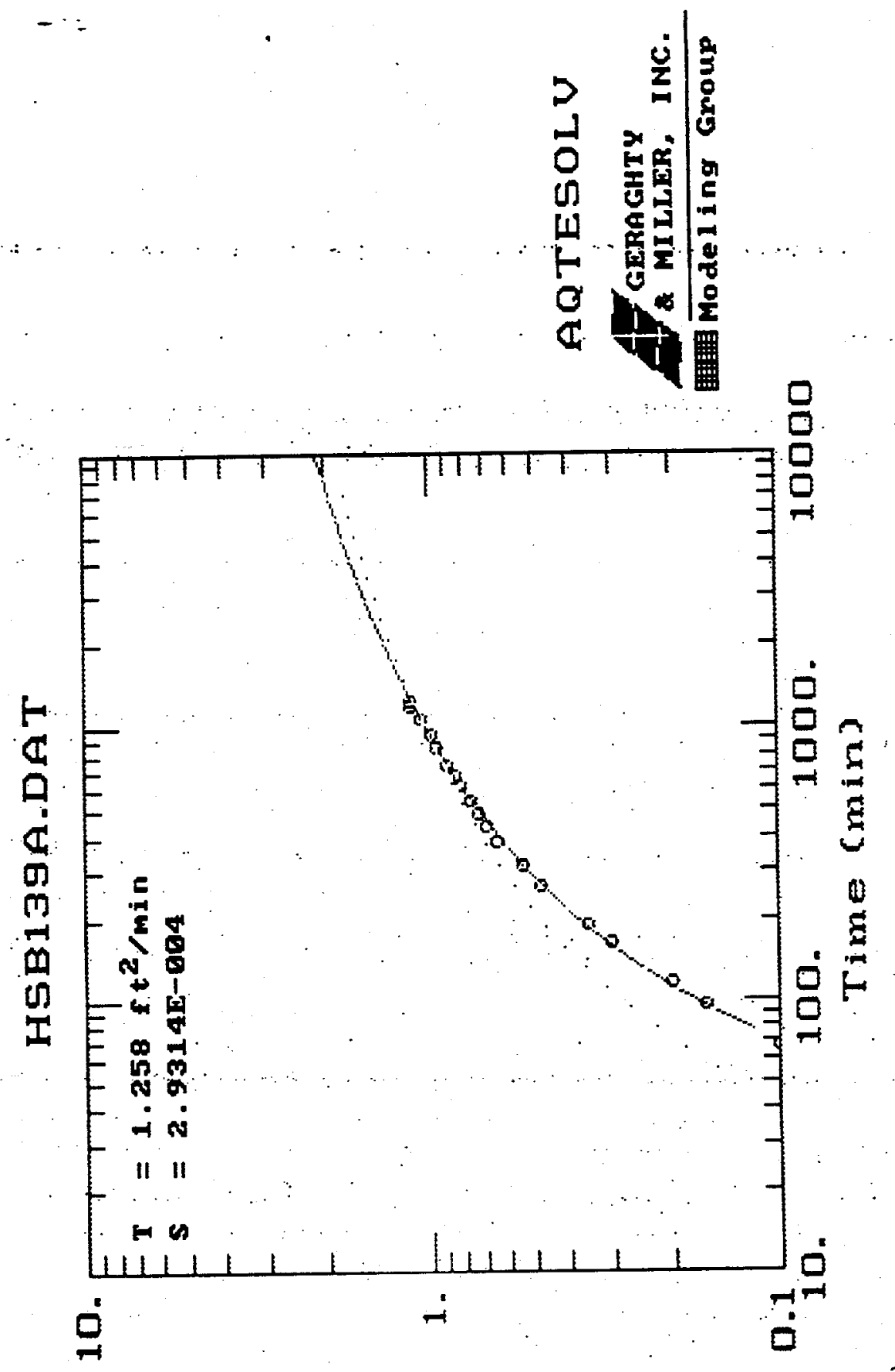

(7f) שMOpMExa 


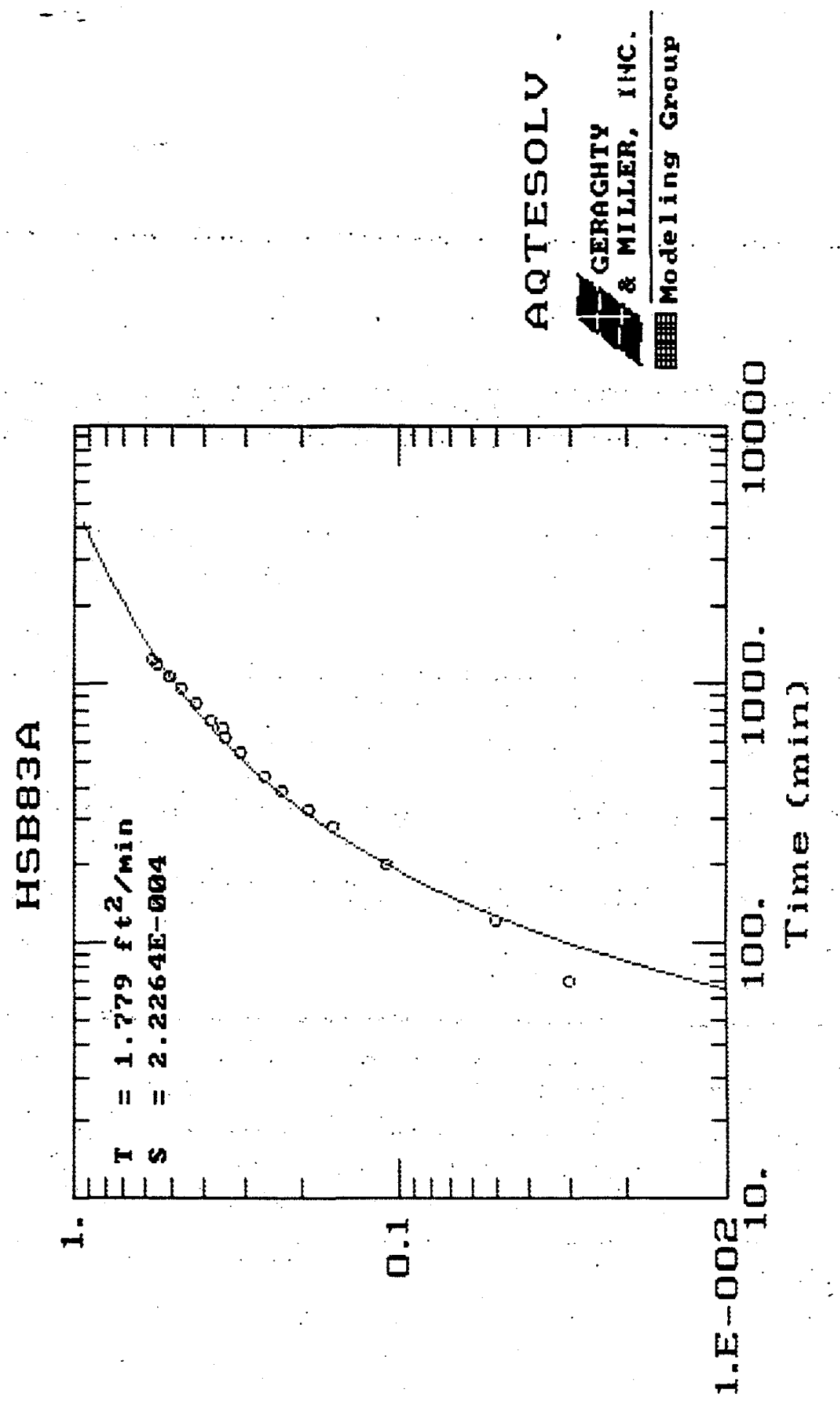

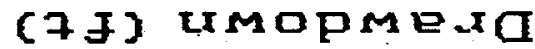


APPENDIX E

$$
\text { AQTESOLV THST TWO }
$$




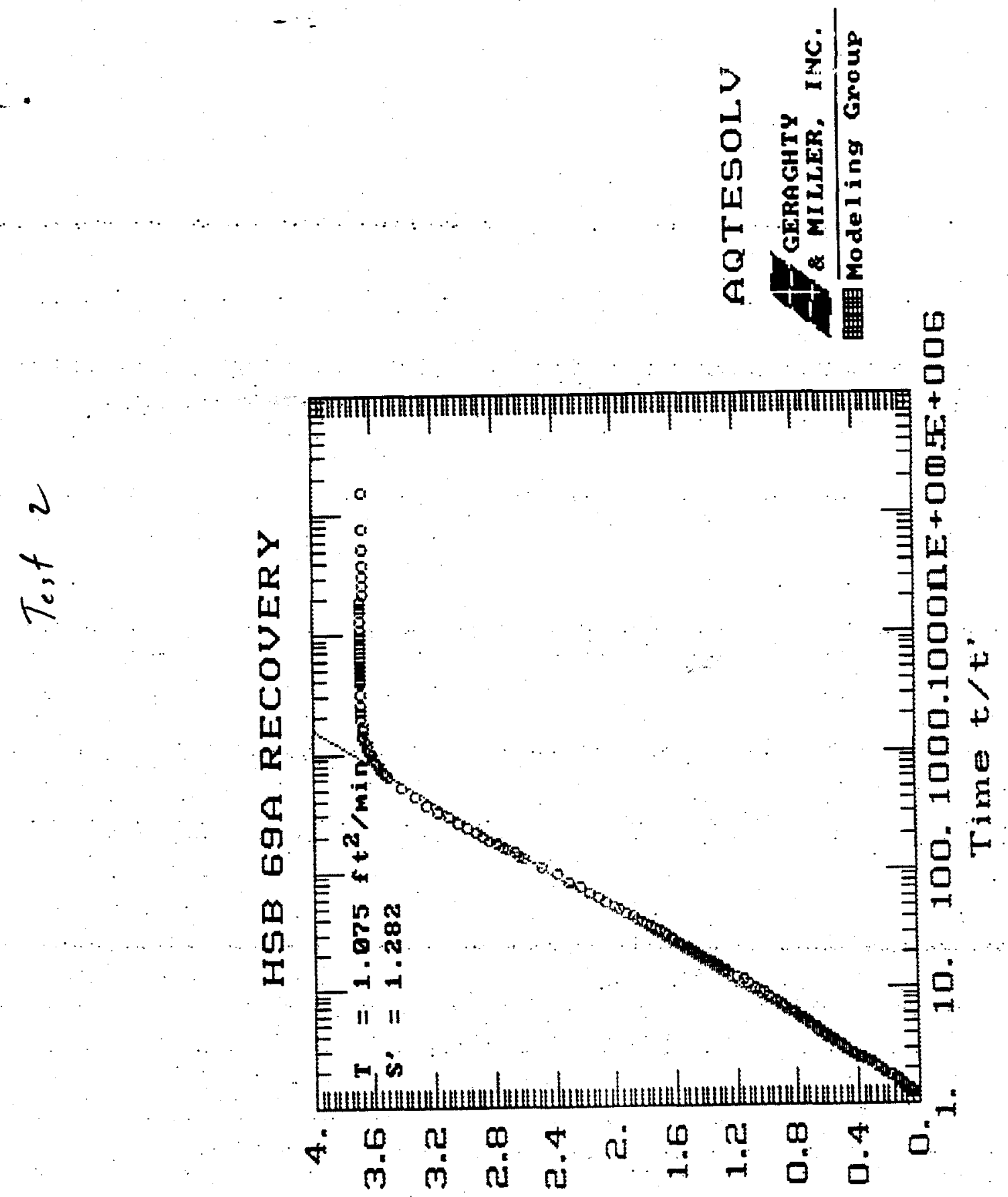

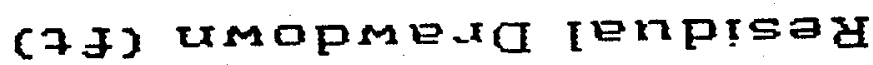




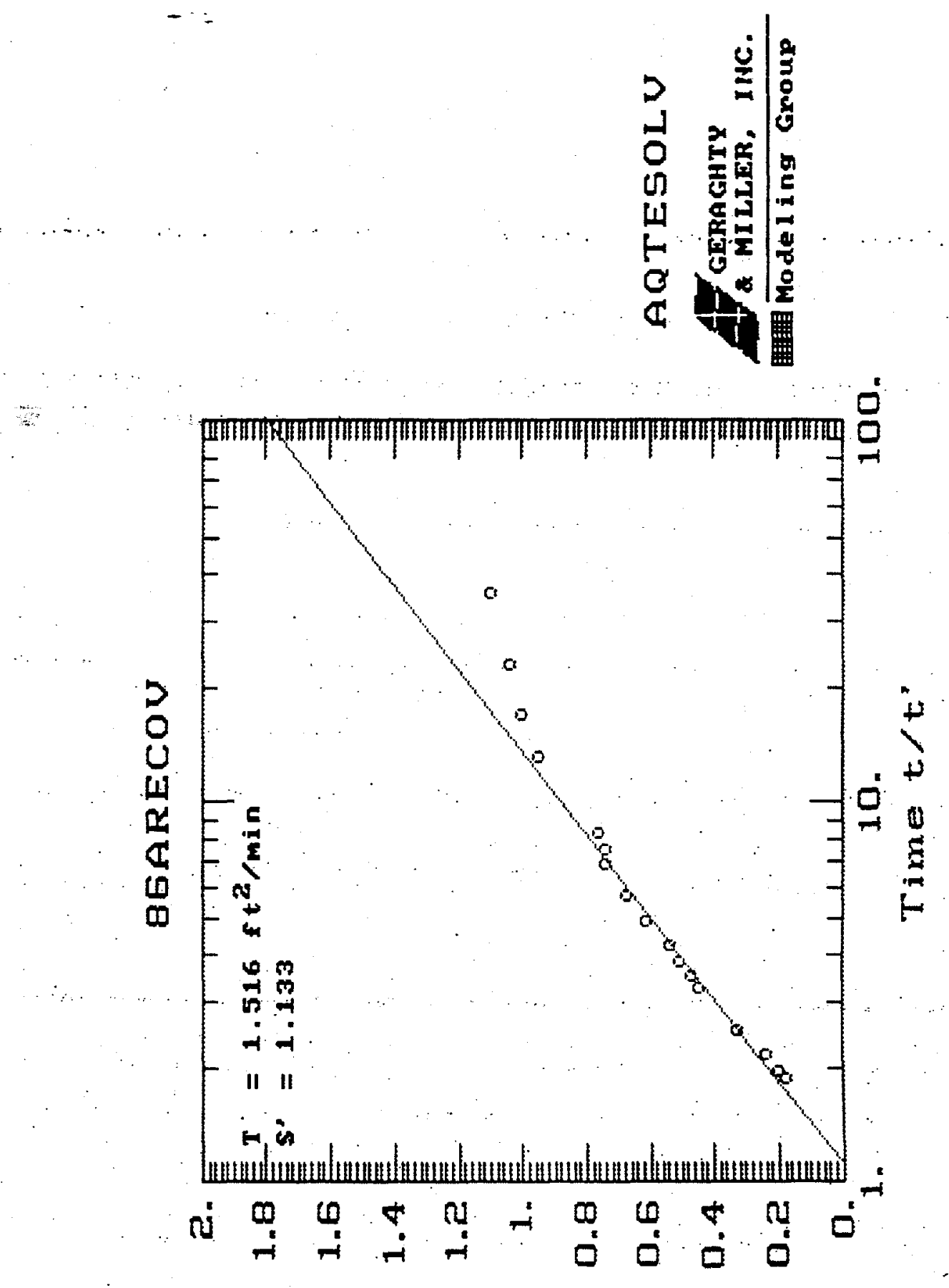

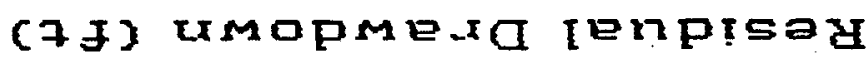




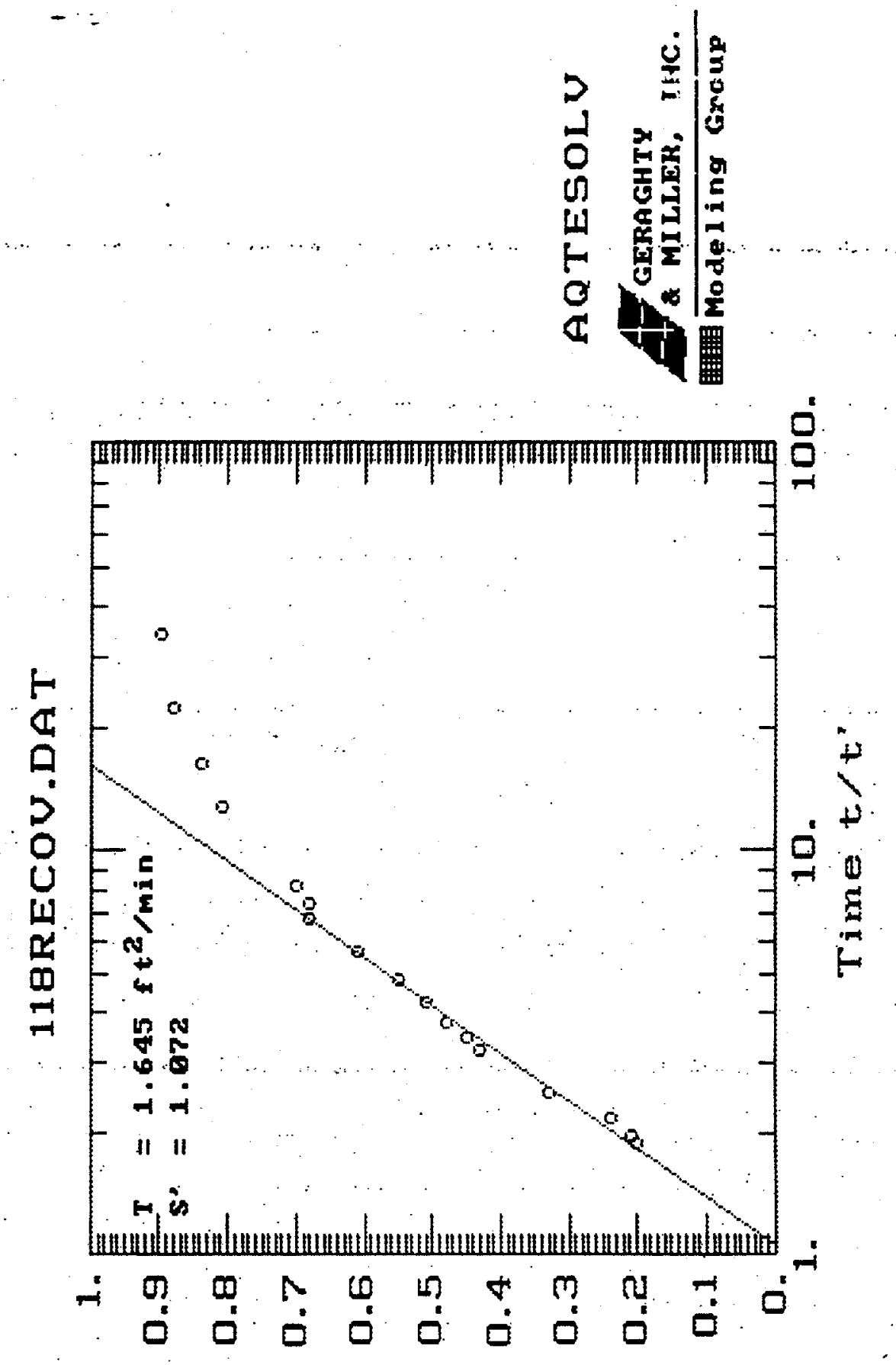

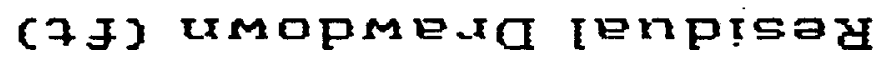




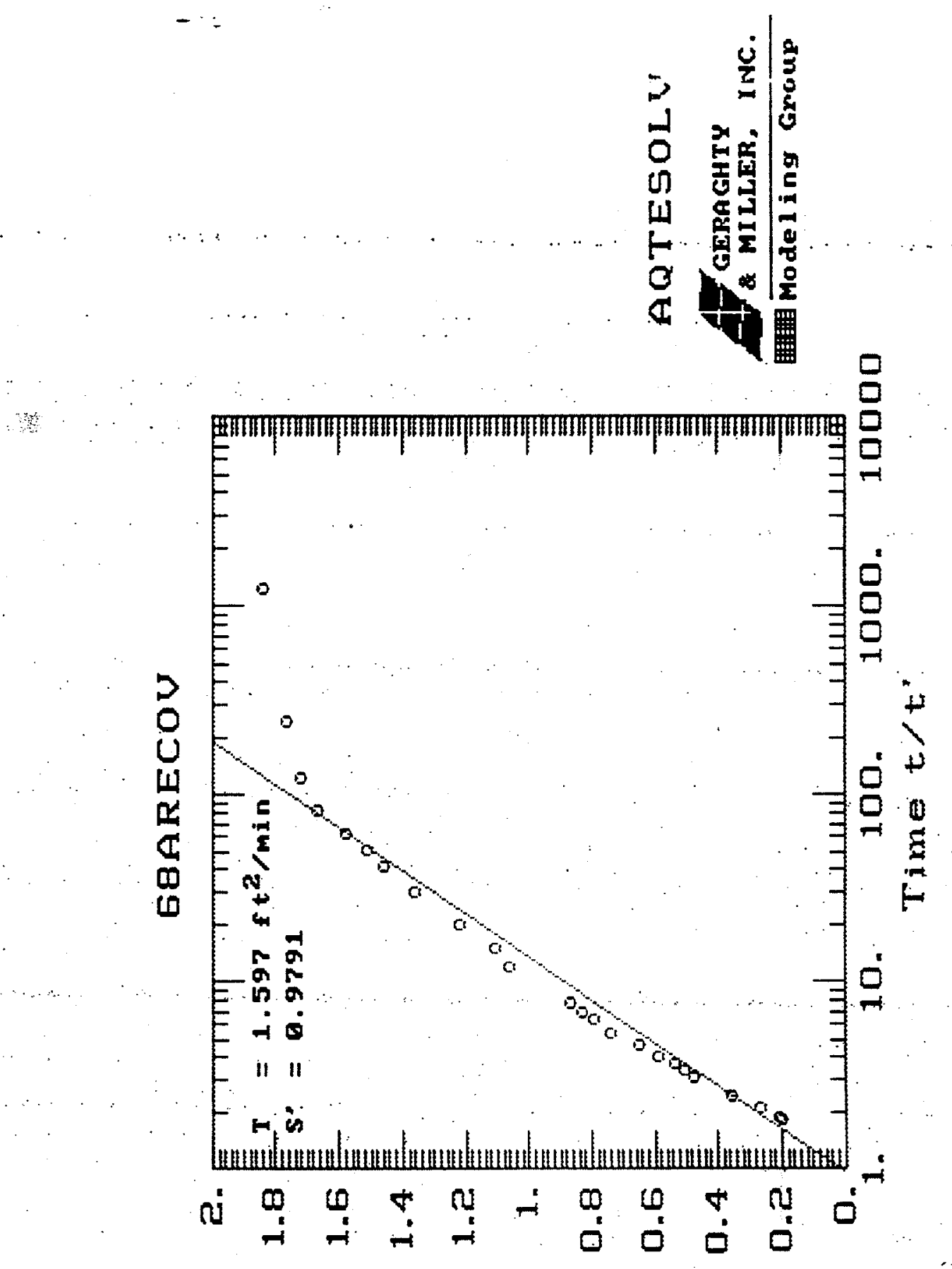

(7) xмmppexa tenptsay 


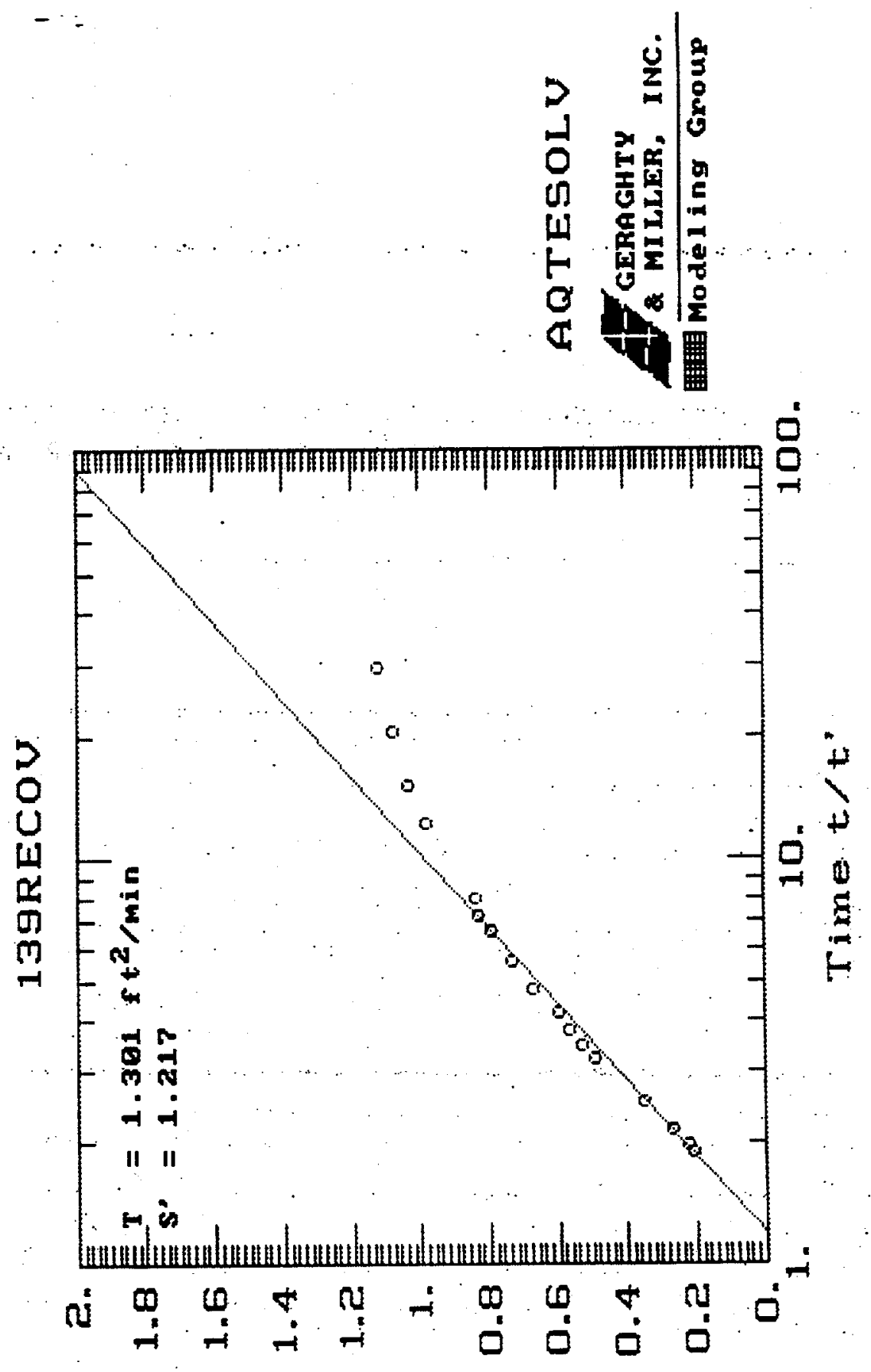

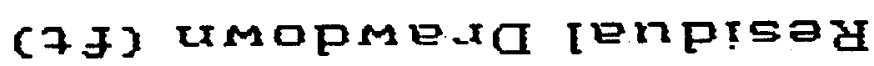




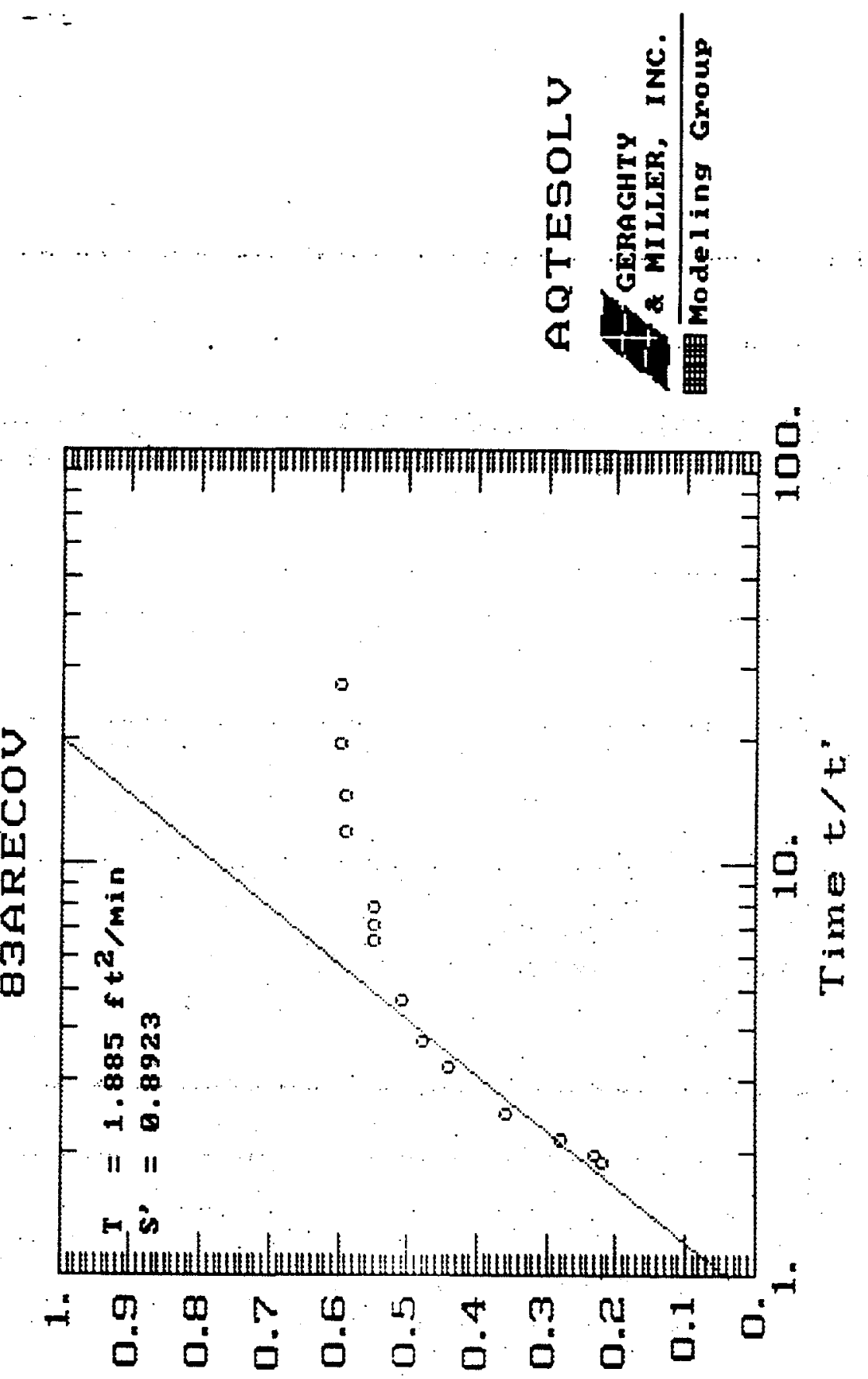

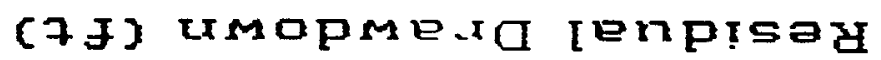


APPENDIX $\dot{\boldsymbol{F}}$

HSB 84A PUMPING TEST

CHEMICAL DATA 
APPENDIX $F$ LEGEND

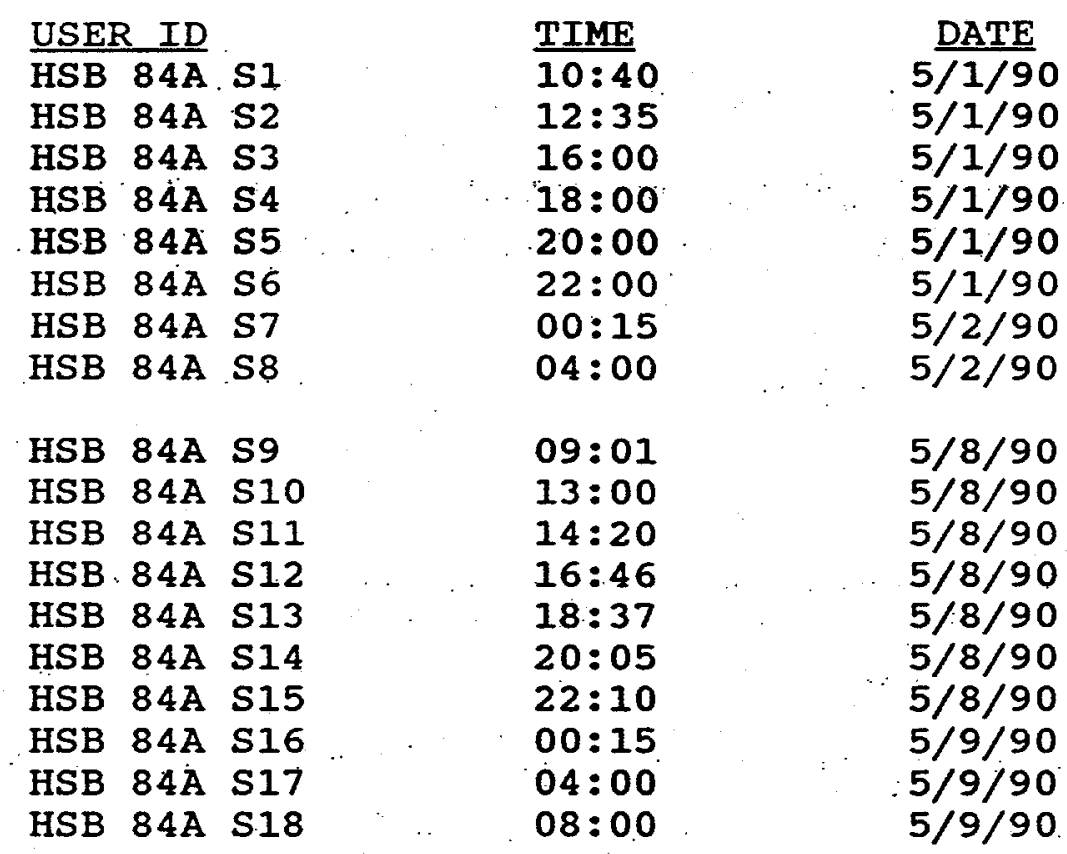


>)> Analytlcal Services: Customer Sanple RESULTS Report $v 1.15 .9<<<<$

Saturday Hay 5, $19903: 0$ an

Group by Subaltter Ordered by Sample Type, User Id, Method, Sequence.

subsitter aldemserug_pu

$158-84 A-51$

ADS SampId: 200042362
Study : H/F GROUND WATER
Addrase intow :

CustomDG \&ASTE HGNT

SanpleType: H/F-LIO-TRITIUM

LoggedBy RHINEHART_RH
Subuissionlds 100009480

Subultoate: $05 / 04 / 90$

LoggerPhone: $\times 5-2034$

Results Section

nethod Results.

iration 1 : TRITIUH PREP

Conponent

TRITIUn

TRITIUH PREP
Analyst: THOMPSDN_MR

Value

$2 / 20$

105

eration 2 : BETA LIO SCINT

ADP FILE/NOTEBDOK REF : BETA LIO SCINT COMMENTS P 189

Analyst: FULMER_GT

Component

Value

TRITIUN

$1.50 \cdot E-3$ UEI/ML

Units

ML.

PACE NUMBER

Units

TEXT
1
$\vdots$
$\mathrm{R} v$

R V

11

A V
11

$\begin{array}{ll}\text { A } & \text { V } \\ 1 & 1\end{array}$

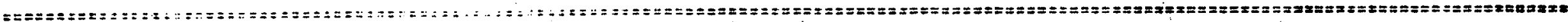


$>>>$ Analytical Services: Customer Sample RESULTS Report $\quad v i .15 .9<<<<$ Group by Subaitter Ordered by Sample Type, User Id, Method, Sequence

Saturday May 5, 1990310 a Subaitter : ALBENESIUS_PW

$5 B-84 A-52$

ADS Sampld: 200042363

Study, I H/F GROUND WATER

Helhod Results.

ration 1 TRITIUA PREP

Address I Unknown

CustomDG : HASTE MGNF

SampleType: H/F-LIQ-TRITIUM

LoggedBy I RHINEHART_RH

Suba issionIds 100009480

Subufloate : 05/04/90

LoggerPhone : $\times 5-2034$

Results Section

Analyst: THOMPSON_MR

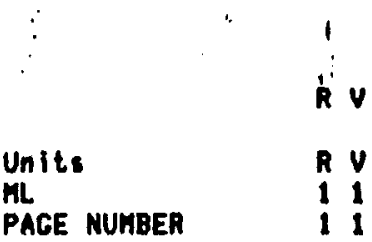

Conponen

TRITIUH PREP

Value
$2 / 20$

105

rat Ion 2 : BETA LIO SCINT ADP FILE/NOTEBODK REF!

BETA LIQ ECINT COMMENTS $P 189$

Analyst: FULMER_GT

Value 


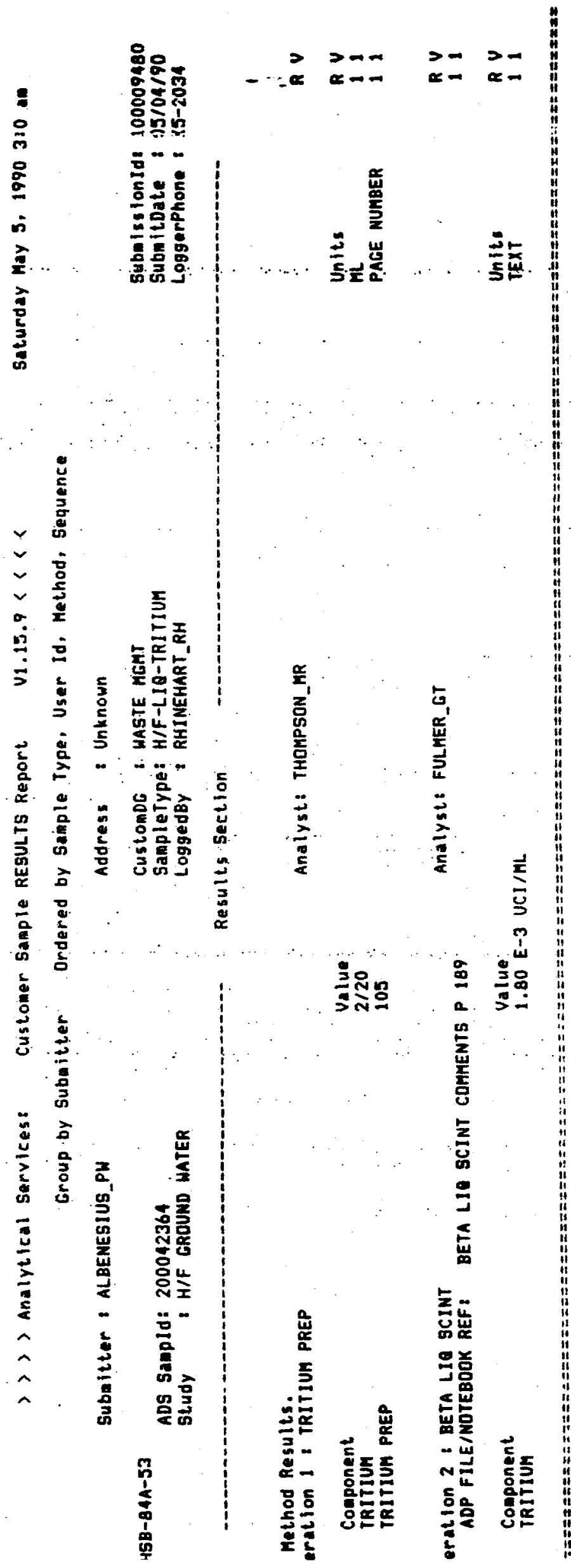


$>>>$ Analytical Services: Customer Sample RESULTS Report. $\quad$ I. $15.9<<<<$

Saturday May 5, 1990 3:0 an

Group by Subvitter Ordered by Sample Type, User Id, Method, Sequence

$158-84 A-54$

ADS Sanpld: 200042365

Study I H/F GROUND WATER
Submitter : ALBENESIUS_PW

Address : Unknown

CustomDG : HASTE MGMT

LoggedBY \& RINEHART RH

subnitbate 05/04/90

LoggerPhone $\times 5-2034$

Results section

Analyst: THOMPSON_MR

i'

iration I TRITIUN PREP

\section{Cosponent}

TRITIUn

TRITIUA PREP

\section{Value}

$2 / 60$

iration 2 : BETA LIG SCINT ADP FILE/NOTEBOOK REF:

BETA LIQ SCINT COHMENTS P 189
Analyst: FULMER_GT

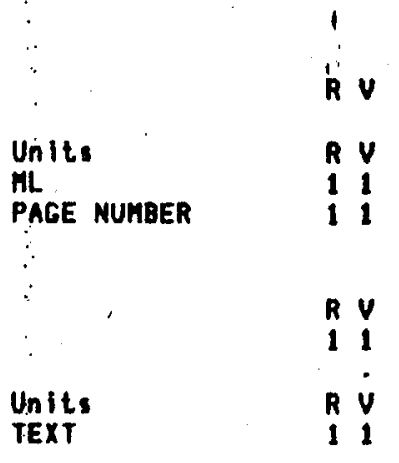

Conponent

Value
$1.89 \mathrm{E}-3 \mathrm{UCI} / \mathrm{ML}$

$\begin{array}{ll}R & V \\ 1 & 1\end{array}$ 


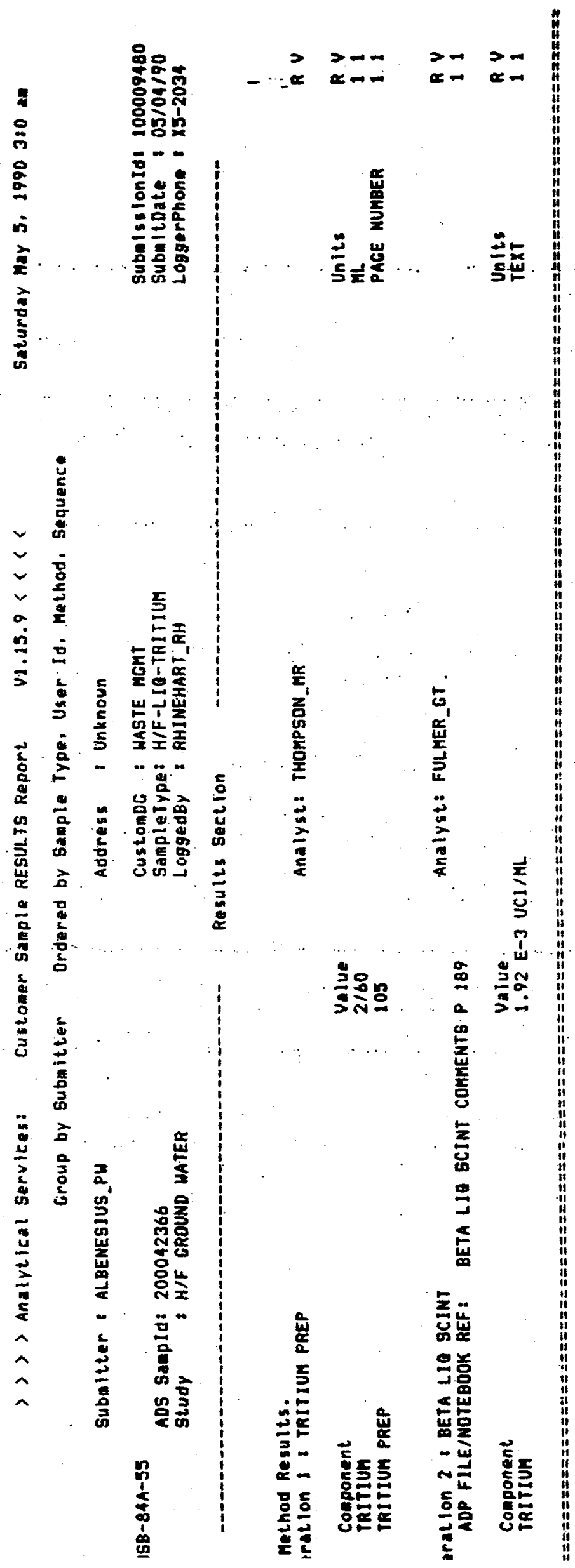


;>> Analytical Services:

Custoner Sample RESULTS Report

V1. $15.9<<<<$

Group by Submitter. Drdered by Sample Type, User Id. Method, Sequence
$45 B-84 A-56$

\section{ADS Sampld: 200042367}

Study : H/F GROUND WATER
Method Results.

eration 1 TRITIUn PREP

\section{Component}

IRITIUA

TRITIUA PREP

\section{Address : Unknown}

Value
$2 / 60$

105

eration 2 : BETA LIG SCINT ADP FILE/NOTEBOOK REFI

BETA LIO SCINT COMMENTS P 189
CustomDG : HASTE MGMT

SampleTYpe: H/F-LIQ-TRITIUH

LoggedBy RHIMEHARI_RH
Results section

Analyst: THOMPSON_MR
Subwission 1d: 100009480 Subuitoate: $05 / 04 / 90$ LoggerPhone $\times 5-2034$

Analyst: FULMER_GT
Units

ML

PAGE NUMBER

R Y

11

R V

11

Units $\quad$ R V

Component

TRITIUH
Value

$1.96 \mathrm{E}-3$ UCI/ML 


\section{>> > Analytical Services: \\ Customer Sample RESULTS Report \\ $v 1.15 .9<<<<$}

Group by subsitter

Drdered by Sanple type. User Id, Method, Sequence

Subwitter : ALBENESIUS_PH

$45 B-84 A-57$

ADS Sampldi 200042368

Study

H/F GRDUND WATER

Method Results.

oration 1 TRITIUn PREP

\section{Component}

TRITIUN

TRITIUN PREP

eration 2 : BETA LIQ SCINT ADP FILEINOTEBODK REF: BETA LIA SCINT COMMENTS P 189
Address Unknown

CustomDG : WASTE MGMT

SampleType: H/F-LIO-TRITIUM

LoggedBy $1 \cdot$ RHINEHART_RH

Results section

Analyst: THOMPSON_MR

Value

$2 / 60$

105

Value
$1.95 \mathrm{E}-3 \mathrm{UCI} / \mathrm{ML}$

Gaturday May 5, 1990380 an

Bubselscionlds 100009480 SubnitDate : O5/04/90 LoggerPhone $\times 5-2034$

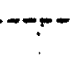
R V

Units

ML

ṔAge number

$\begin{array}{ll}R & V \\ 1 & 1\end{array}$

11

Analyst: FULHER_GT 
$>>>$ Analytical Services: Customer Sample RESULTS Report

Group by Submitter. Ordered by Sample Type, User Id, Method, Sequence

Subaitter : ALBENESIUS_PH

HSB $-84 A-5 B$

ADS Sampld: 200042369

Study : H/F GROUND HATEER

\section{Address : Unknown}

SampleType: H/F-LIQ-TRITIUM

Results Section

\section{Value}

2160

TRITIUn

TRITIUN PREP perat fon 2 : BETA LIO SCINT ADP FILEINOTEBDOK REF: BETA LIQ SCINT COMMENTS P 189
CustomDG WASTE MGMT

LoggedBy : RHINEHART_RH

Analysl: THOMPSON_MR

Subw Issionlds 100009480

SubaitDate $103 / 04 / 90$

Loggerphon : X5-2034

R V

Units

It

PAGE NUMBER

and

Analyet: FULHER_GT 


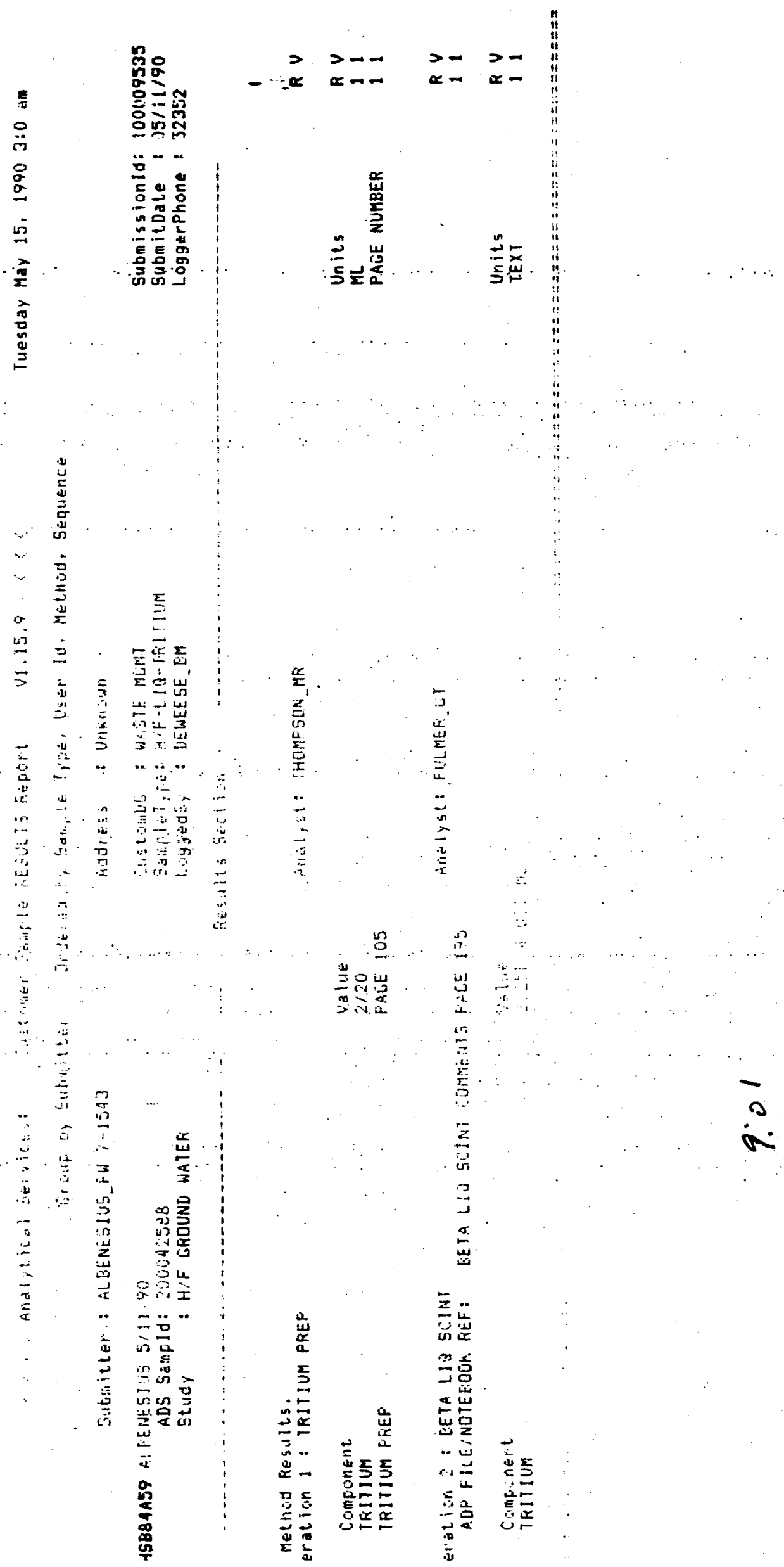




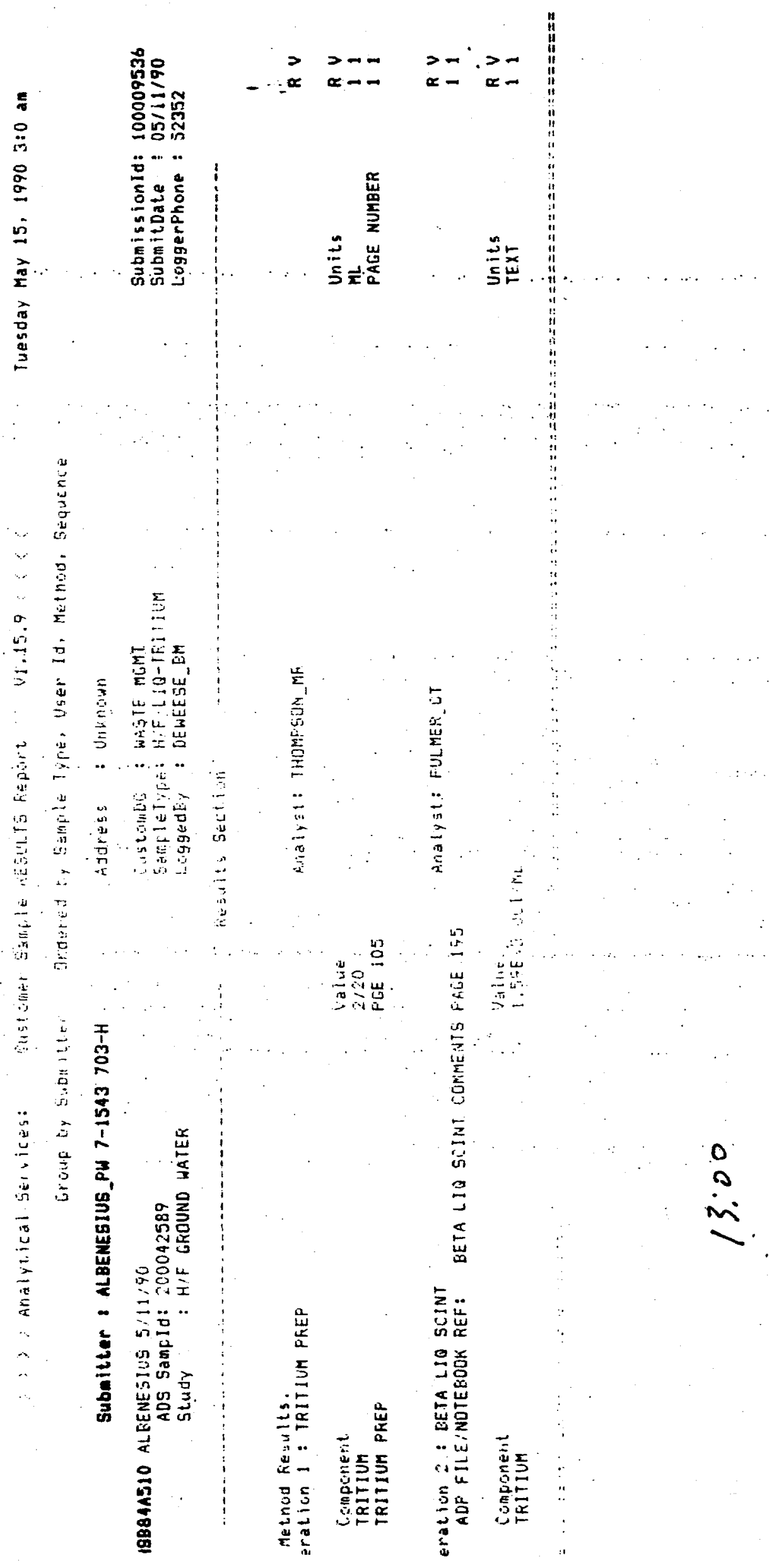




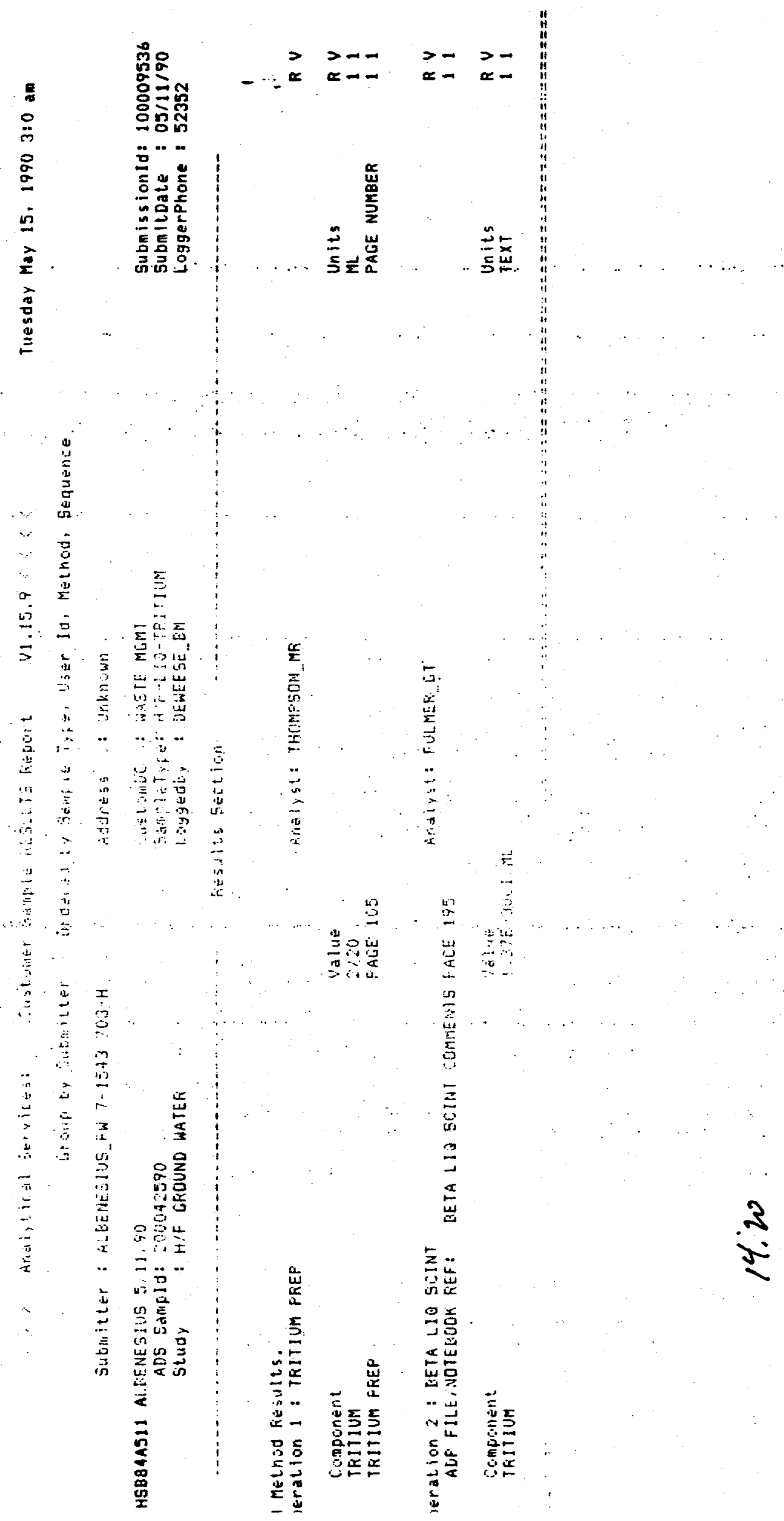




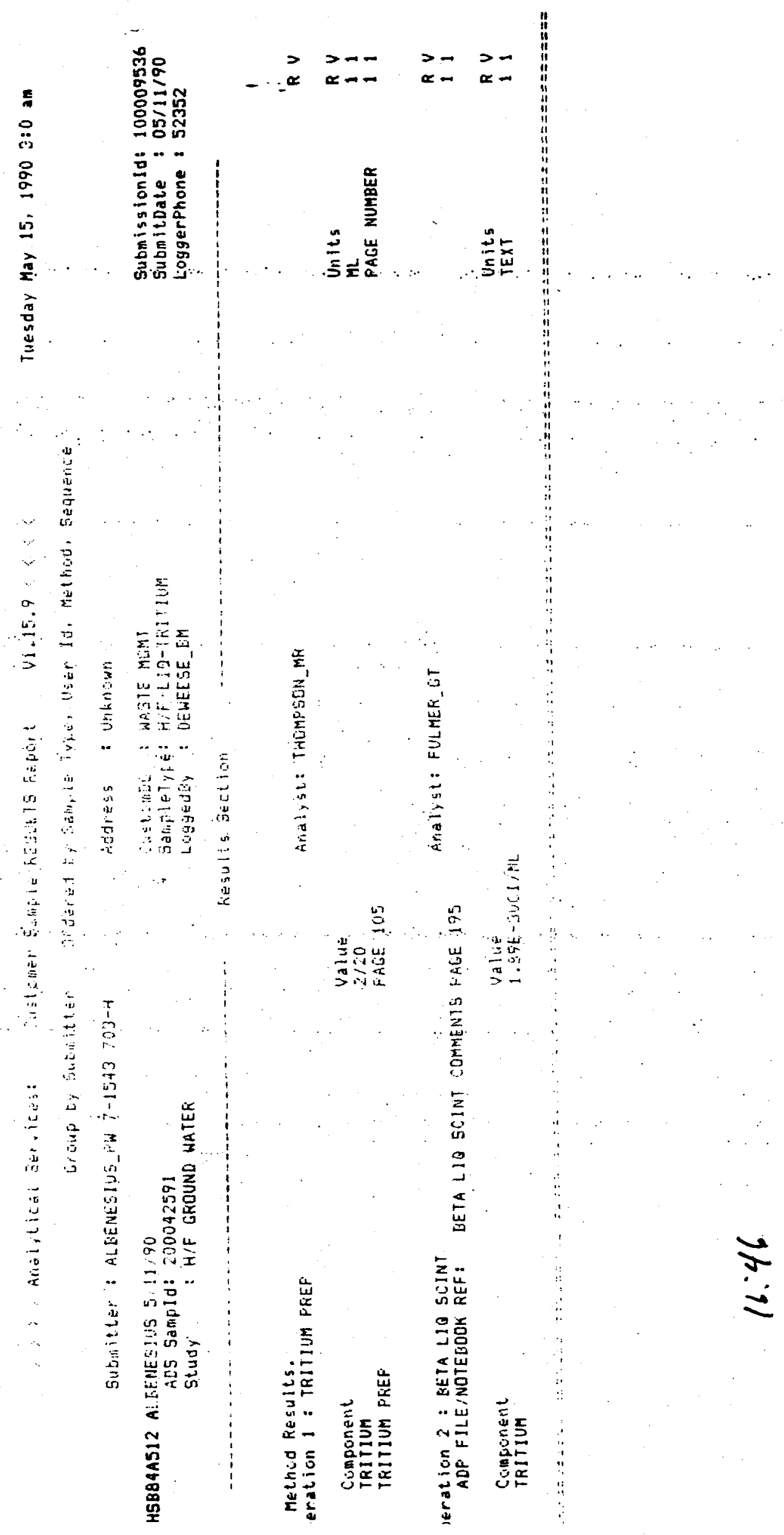




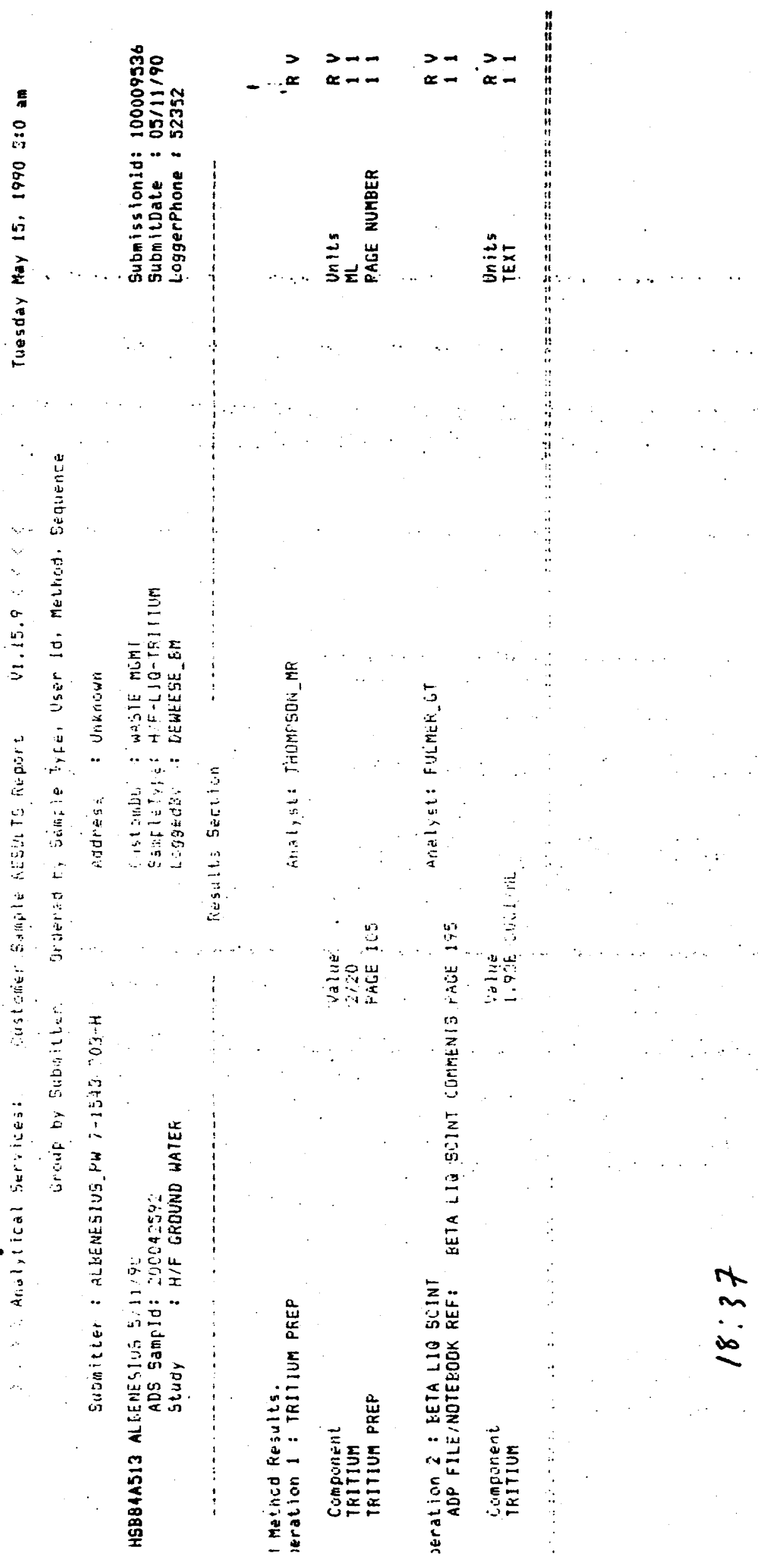


Subinitter : ALEENESIUS_FW 7-1543703-H

ISB84A514 ALLENES1U5 $5: 11 / 90$

ADS Sampld: 200042593

Study
Address : Unknown

EASTUDOS : WRETE MEMT

SampleTYFe: H/F-LIQ-TFITIUR

LOgGEdB; : DEWEESE: EM

Fesult.s Section

AnAlyst: THOMFSOH_MR

Via
$21 \% 0$

2720

FALE IIS
Submissionld: 100009536 SubmitDate : $05 / 11 / 90$ Loggerphone: $\mathbf{5 2 3 5 2}$

\section{Methed Results.}

iration 1 : TRITIUM PREF

Component

IRITIUU FREP

Eration 2 : EETA LIQ SCINT ADF FILE/NOTEEOOK REF:

Component

TFITIUM

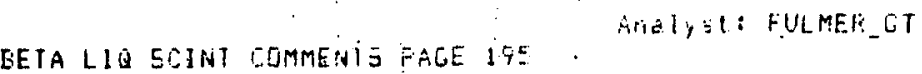

Valus

$1.90 \mathrm{O} 3 \mathrm{BLO} / \mathrm{MI}$

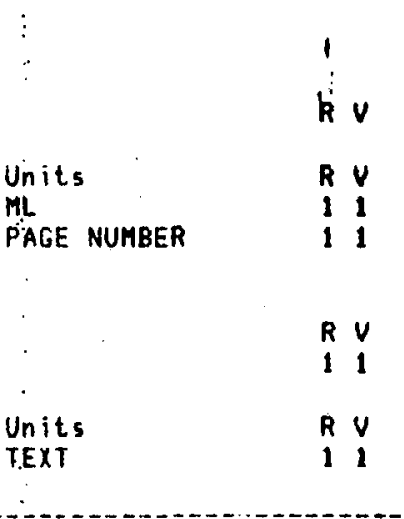

$20: 05$ 


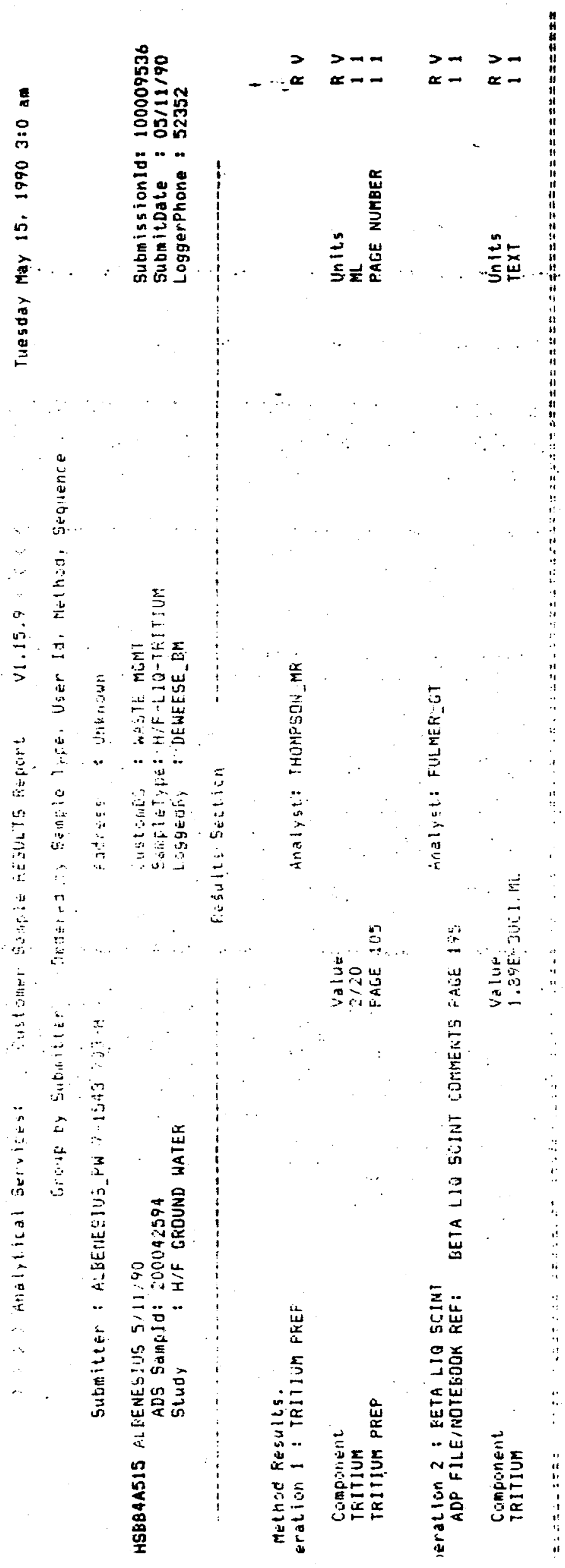

$\stackrel{3}{N}$ 
Sutmitter : ALEENESIUS_FW \%-1373, $70,-H$

HSB84A516 ALLENESIULS $5 / 11 / 90$

ADS Sampld: 200042595

Study : H/F GROUND WATER

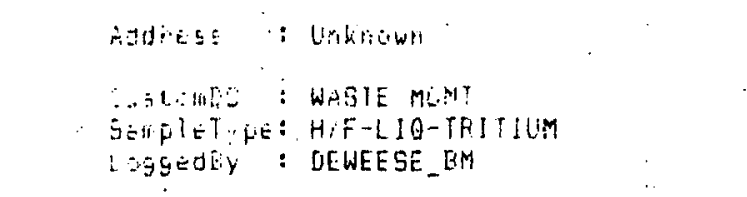

Fesults section. i Method Results.

ieration 1 : TRITIUM FREP

\section{Companent}

TRITIUM

IFITIUM FREP

Jeration 2 : BETA L19 SCINT ADF FILE, NOTEBDDK REF:

BETA. LIQ SEINT CUMAENTS FACE I 95

\section{Aral,st: THUMFSOH_MF}

Value

F.ACE 105

Componert

TRITIUA

\section{Valute}

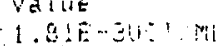

Ariaiyst: FULMEF ET

Submissionld: 100009536 SubmitDate : $05 /: 1 / 90$

Loggerphone 52352 


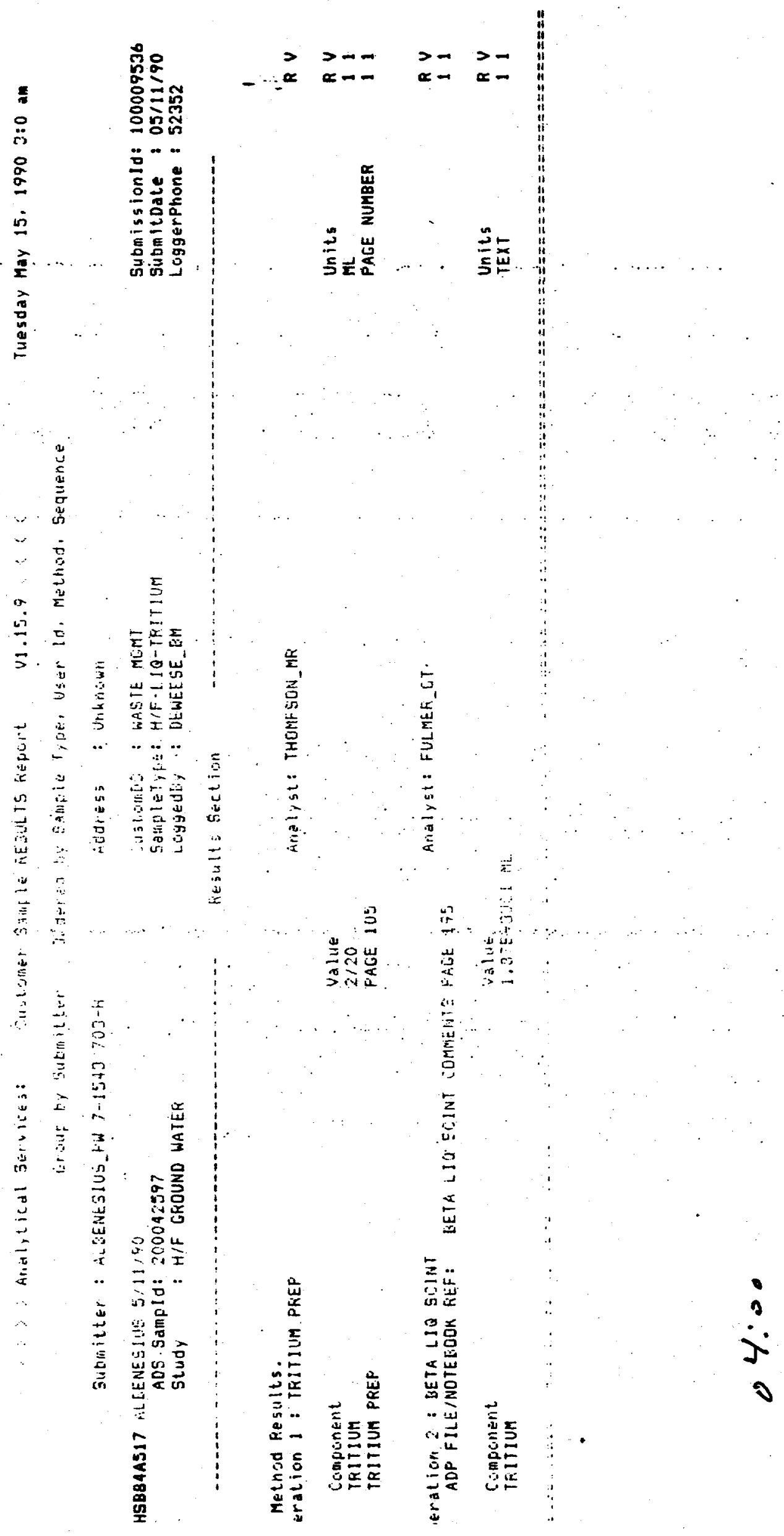




\section{APPENDIX $G$}

HSB $84 \mathrm{~A}$ - PUMPING TEST

PRE-TEST WATER LEVEL DATA 
HSB $84 A$

AFRII 27, 1990

PRE-TEST WATER LEVEL CHANGES

ELAPSED TIME

CHANGE

0.0000

0.000

.15 .0000

$-0.015$

30.0000

$-0.015$

45.0000

$-0.015$

60.0000

$-0.015$

75.0000

$-0.031$

90.0000

$-0.031$

105.000

$-0.031$

120.000

-0.047 .

135.000

$-0.047$

150.000

$-0.047$

165.000

$-0.047$

180.000

195.000

210.000

225.000

$240: 000$

255.000

270.000

285.000

300.000

315.000

330.000

345.000

360.000

375.000

390.000

405.000

$-0.063$

$-0.063$

$-0.063$

$-0.063$

$-0.078$

$-0.078$

$-0.078$

$-0.094$

$-0.094$

$-0.094$

$-0.110$

$-0.110$

$-0.110$

$-0.110$

$-0.110$

$-0.110$

420.000

$-0.126$

435.000

$-0.126$

450.000

$-0.126$

465.000

$-0.126$

480.000

$-0.126$

495.000

$-0.126$

510.000

$-0.141$

525.000

540.000

555.000

570.000

585.000

600.000

615.000

630.000

$-0.141$

$-0.141$

$-0.141$

$-0.141$

$-0.141$

$-0.141$

$-0.141$

$-0.141$

645.000

$-0.141$

660.000

$-0.141$

675.000

$-0.141$

690.000

$-0.157$

705.000

$-0.157$

720.000

$-0.157$ 


$\begin{array}{ll}735.000 & -0.157 \\ 750.000 & -0.157 \\ 765.000 & -0.157 \\ 780.000 & -0.157 \\ 795.000 & -0.157 \\ 810.000 & -0.157 \\ 825.000 & -0.157 \\ 840.000 & -0.157 \\ 855.000 & -0.157 \\ 870.000 & -0.157 \\ 885.000 & -0.157 \\ 900.000 & -0.157 \\ 915.000 & -0.157 \\ 930.000 & -0.157 \\ 945.000 & -0.157 \\ 960.000 & -0.157 \\ 975.000 & -0.157 \\ 990.000 & -0.157 \\ 1005.00 & -0.157 \\ 1020.00 & -0.157 \\ 1035.00 & -0.157 \\ 1050.00 & -0.157 \\ 1065.00 & -0.141 \\ 1080.00 & -0.157 \\ 1095.00 & -0.157 \\ 1110.00 & -0.157 \\ 1125.00 & -0.141 \\ 1140.00 & -0.157 \\ 1155.00 & -0.157 \\ 1170.00 & -0.141 \\ 1185.00 & -0.141 \\ 1200.00 & -0.141 \\ 1215.00 & -0.141 \\ 1230.00 & -0.157 \\ 1245.00 & -0.157 \\ 1260.00 & -0.157 \\ 1275.00 & -0.141 \\ 1290.00 & -0.141 \\ 1305.00 & -0.141 \\ 1320.00 & -0.157 \\ 1335.00 & -0.157 \\ 1350.00 & -0.141 \\ 1365.00 & -0.141 \\ 1380.00 & -0.141 \\ 1395.00 & -0.141 \\ 1410.00 & -0.141 \\ 1425.00 & -0.141 \\ 1440.00 & -0.141 \\ 1455.00 & -0.141 \\ 1470.00 & \\ 1485.00 & -0.141 \\ 1500.00 & -0.141 \\ 1515.00 & -00\end{array}$




$\begin{array}{ll}1545.00 & -0.141 \\ 1560.00 & -0.141 \\ 1575.00 & -0.157 \\ 1590.00 & -0.141 \\ 1605.00 & -0.141 \\ 1620.00 & -0.157 \\ 1635.00 & -0.157 \\ 1650.00 & -0.157 \\ 1665.00 & -0.157 \\ 1680.00 & -0.157 \\ 1695.00 & -0.157 \\ 1710.00 & -0.157 \\ 1725.00 & -0.173 \\ 1740.00 & -0.205 \\ 1755.00 & -0.205 \\ 1770.00 & -0.205 \\ 1785.00 & -0.205 \\ 1800.00 & -0.205 \\ 1815.00 & -0.220 \\ 1830.00 & -0.220 \\ 1845.00 & -0.236 \\ 1860.00 & -0.236 \\ 1875.00 & -0.236 \\ 1890.00 & -0.236 \\ 1905.00 & -0.236 \\ 1920.00 & -0.236 \\ 1935.00 & -0.236 \\ 1950.00 & -0.252 \\ 1965.00 & -0.252 \\ 1980.00 & -0.252 \\ 1995.00 & -0.252 \\ 2010.00 & -0.252 \\ 2025.00 & -0.252 \\ 2040.00 & -0.252 \\ 2055.00 & -0.252 \\ 2070.00 & -0.252 \\ 2085.00 & -0.252 \\ 2100.00 & -0.252 \\ 2115.00 & -0.252 \\ 2130.00 & -0.252 \\ 2145.00 & -0.252 \\ 2160.00 & -0.252 \\ 2175.00 & -0.252 \\ 2190.00 & -0.252 \\ 2205.00 & -0.252 \\ 2220.00 & -0.252 \\ 2235.00 & -0.252 \\ 2250.00 & -0.252 \\ 2265.00 & -0.252 \\ 2280.00 & -0.252 \\ 2395.00 & -0.252 \\ 2325.00 & -0.252 \\ 2340.00 & -0.252 \\ & -0.252\end{array}$




$\begin{array}{ll}2355.00 & -0.252 \\ 2370.00 & -0.252 \\ 2385.00 & -0.252 \\ 2400.00 & -0.252 \\ 2415.00 & -0.252 \\ 2430.00 & -0.252 \\ 2445.00 & -0.252 \\ 2460.00 & -0.252 \\ 2475.00 & -0.252 \\ 2490.00 & -0.236 \\ 2505.00 & -0.236 \\ 2520.00 & -0.220 \\ 2535.00 & -0.220 \\ 2550.00 & -0.220 \\ 2565.00 & -0.220 \\ 2580.00 & -0.220 \\ 2595.00 & -0.205 \\ 2610.00 & -0.220 \\ 2625.00 & -0.205 \\ 2640.00 & -0.189 \\ 2655.00 & -0.205 \\ 2670.00 & -0.189 \\ 2685.00 & -0.189 \\ 2700.00 & -0.189 \\ 2715.00 & -0.189 \\ 2730.00 & -0.189 \\ 2745.00 & -0.189 \\ 2760.00 & -0.189 \\ 2775.00 & -0.189 \\ 2790.00 & -0.189 \\ 2805.00 & -0.189 \\ 2820.00 & -0.189 \\ 2835.00 & -0.189 \\ 2850.00 & -0.189 \\ 2865.00 & -0.189 \\ 2880.00 & -0.189 \\ 2895.00 & -0.189 \\ 2910.00 & -0.189 \\ 2925.00 & -0.189 \\ 2940.00 & -0.189 \\ 2955.00 & -0.189 \\ 2970.00 & -0.189 \\ 2985.00 & -0.189 \\ 3000.00 & -0.189 \\ 3015.00 & -0.205 \\ 3030.00 & -0.189 \\ 3045.00 & -0.205 \\ 3060.00 & -0.205 \\ 3075.00 & -0.205 \\ 31090.00 & -0.205 \\ 3120.00 & -0.220 \\ 3150.00 & \end{array}$




\section{HEB $84 B$}

\section{ARPII 27, 1930}

PRE-TEST WATER LEVEL CHANGES

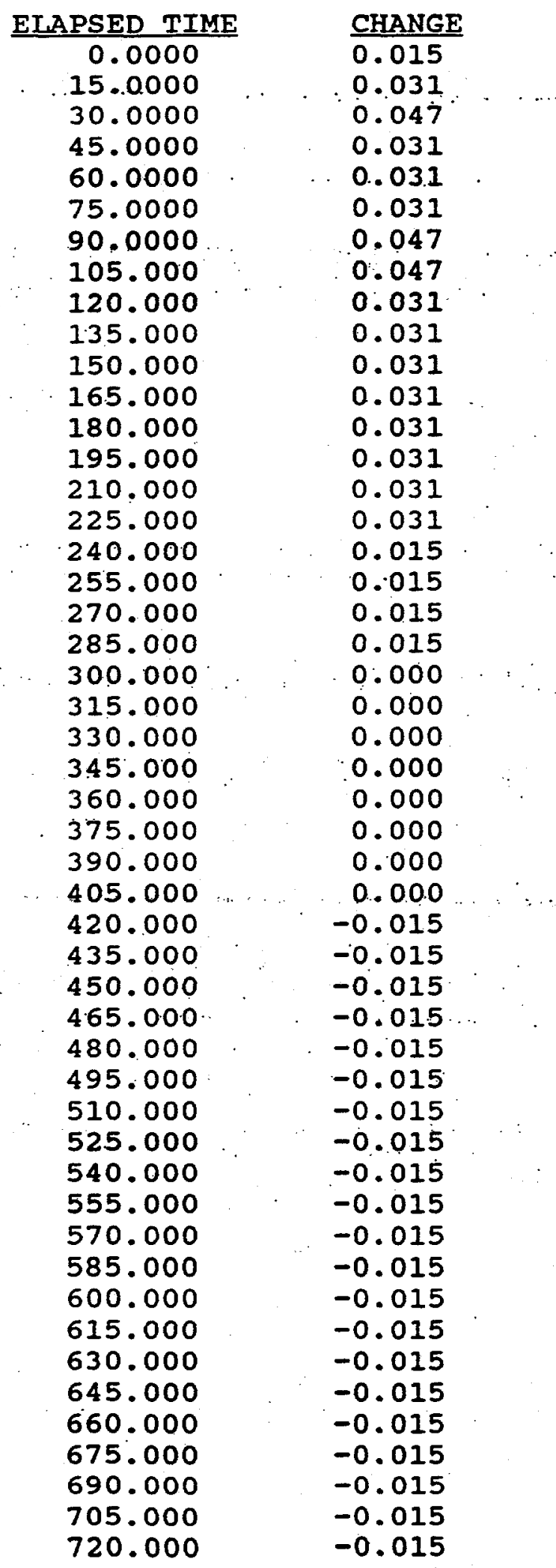




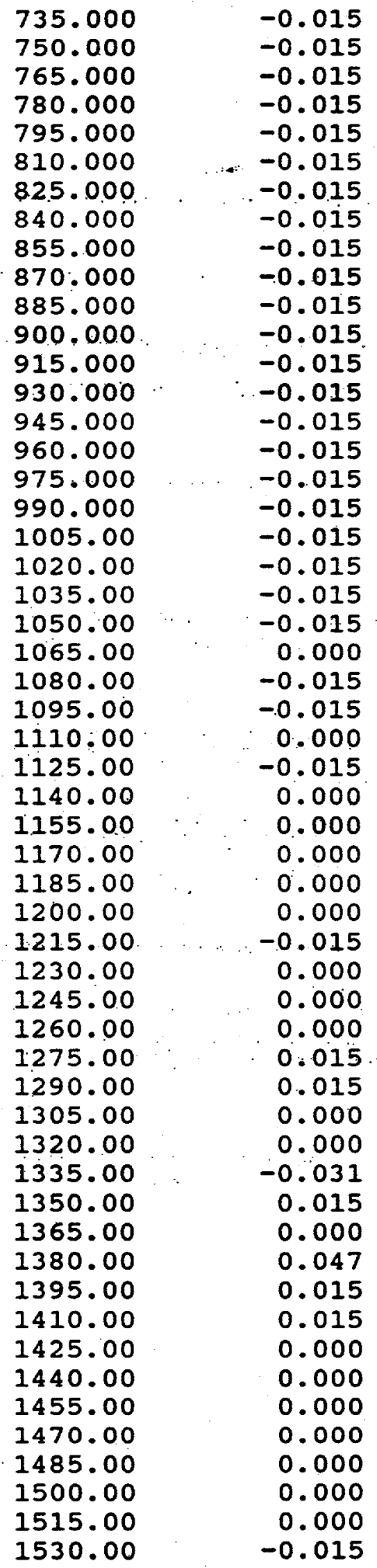




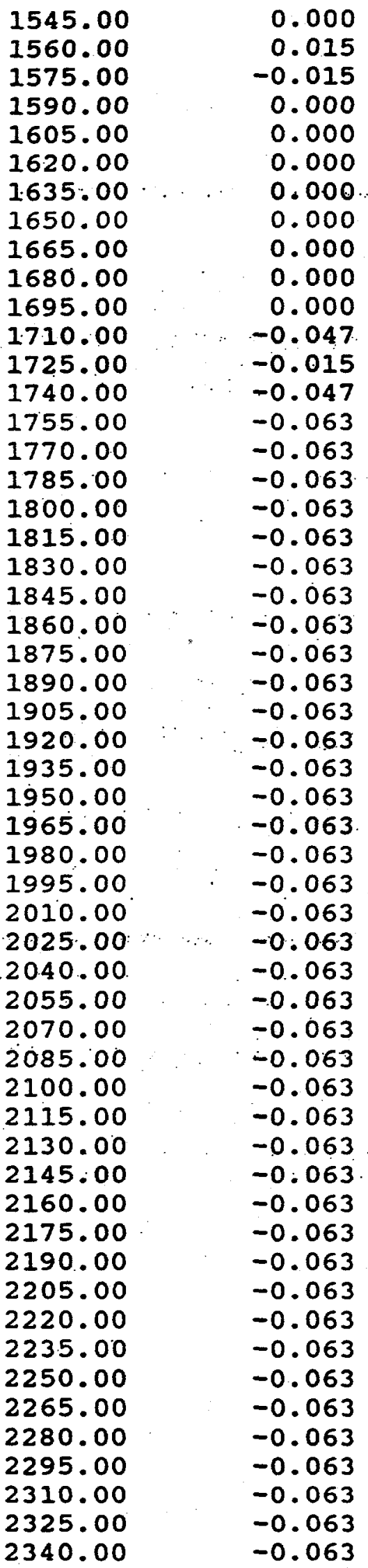




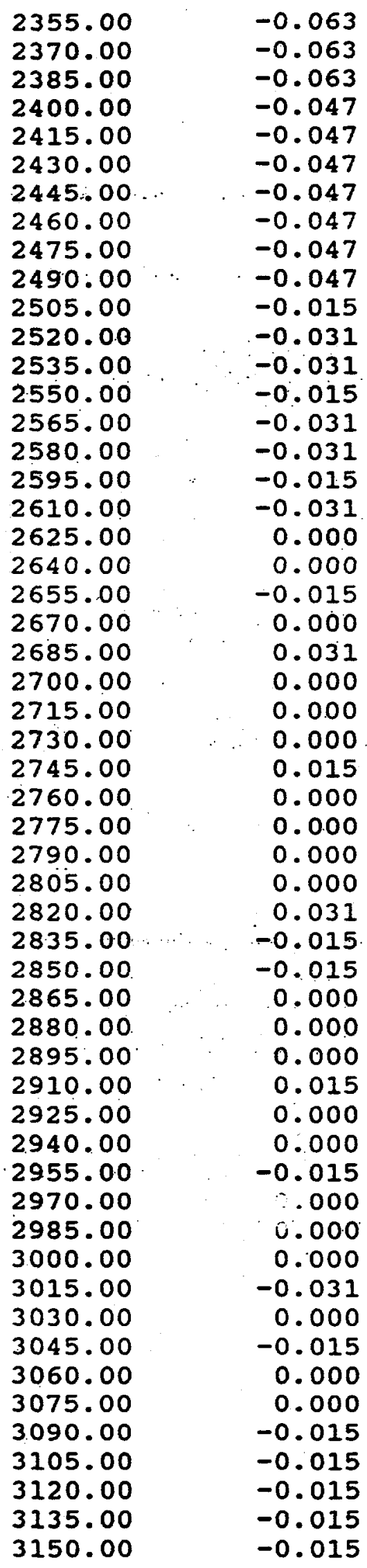




$\begin{array}{ll}3165.00 & -0.015 \\ 3180.00 & -0.015 \\ 3195.00 & -0.031 \\ 3210.00 & -0.031 \\ 3225.00 & -0.031 \\ 3240.00 & -0.031 \\ 3255.00 & -0.031 \\ 3270.00 & -0.031 \\ 3285.00 & -0.031 \\ 3300.00 & -0.031 \\ 3315.00 & -0.031 \\ 3330.00 & -0.047 \\ 3345.00 & -0.047 \\ 3360.00 & -0.047 \\ 3375.00 & -0.047 \\ 3390.00 & -0.047 \\ 3405.00 & -0.047 \\ 3420.00 & -0.047 \\ 3435.00 & -0.047 \\ 3450.00 & -0.047 \\ 3465.00 & -0.047 \\ 3480.00 & -0.047 \\ 3495.00 & -0.047 \\ 3510.00 & -0.047 \\ 3525.00 & -0.047 \\ 3540.00 & -0.047 \\ 3555.00 & -0.047 \\ 3570.00 & -0.047 \\ 3585.00 & -0.047 \\ 3600.00 & -0.047 \\ 3615.00 & -0.047 \\ 3630.00 & -0.047 \\ 3645.00 & -0.063 \\ 3660.00 & -0.047 \\ 3675.00 & -0.063 \\ 3690.00 & -0.047 \\ 3705.00 & -0.047 \\ 3720.00 & -0.047 \\ 3735.00 & -0.047 \\ 3750.00 & -0.015 \\ 3765.00 & -0.047 \\ 3780.00 & -0.047 \\ 3795.00 & -0.047 \\ 3810.00 & -0.047 \\ 3825.00 & -0.047 \\ 3840.00 & -0.047 \\ 3855.00 & -0.031 \\ 3870.00 & -0.031 \\ 3885.00 & -0.031 \\ 3900.00 & -000 \\ 3915.00 & \\ 3930.00 & -0.015 \\ 3945.00 & \end{array}$


$3975.00 \quad 0.000$ 


\section{HSB $84 C$ \\ AFRII 27, 1990 \\ PRE-TEST WATER LEVEL CHANGES}

ELAPSED TIME

0.0000

.15 .0000

30.0000

45.0000

60.0000

75.0000

90.0000

105.000

120.000

135.000

150.000

165.000

180.000

195.000

210.000

225.000

240.000

255.000

270.0 .00

285.000

300.000

315.000

330.000

345.000

360.000

375.000

390.000

405.000

420.000

435.000

450.000

465.000

480.000

495.000

510.000

525.000

540.000

555.000

570.000

585.000

600.000

615.000

630.000

645.000

660.000

675.000

690.000

705.000

720.000
CHANGE

0.047

0.031

0.047

0.015

0.015

0.031

0.031

0.031

0.015

0.015

0.015

0.031

0.015

0.031

0.015

0.015

0.015

0.015

0.015

0.000

0.000

0.000

0.000

0.000

0.000

0.000

0.000

0.000

$-0.015$

$-0.015$

$-0.015$

$-0.015$

$-0.015$

$-0.015$

$-0.015$

$-0.015$

$-0.015$

$-0.015$

$-0.015$

$-0.015$

$-0.015$

$-0.015$

$-0.015$

$-0.015$

$-0.015$

$-0.015$

$-0.015$

$-0.015$

$-0.015$ 


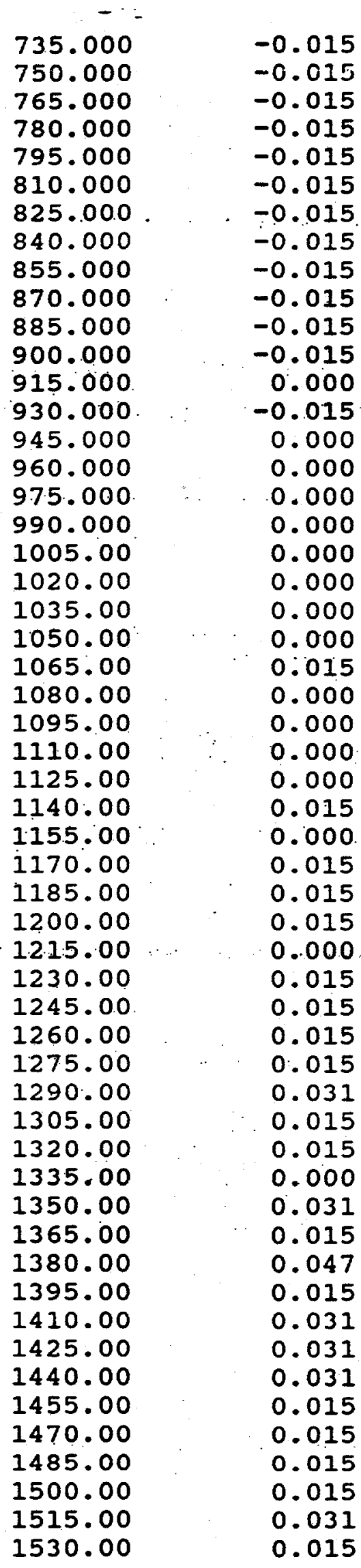




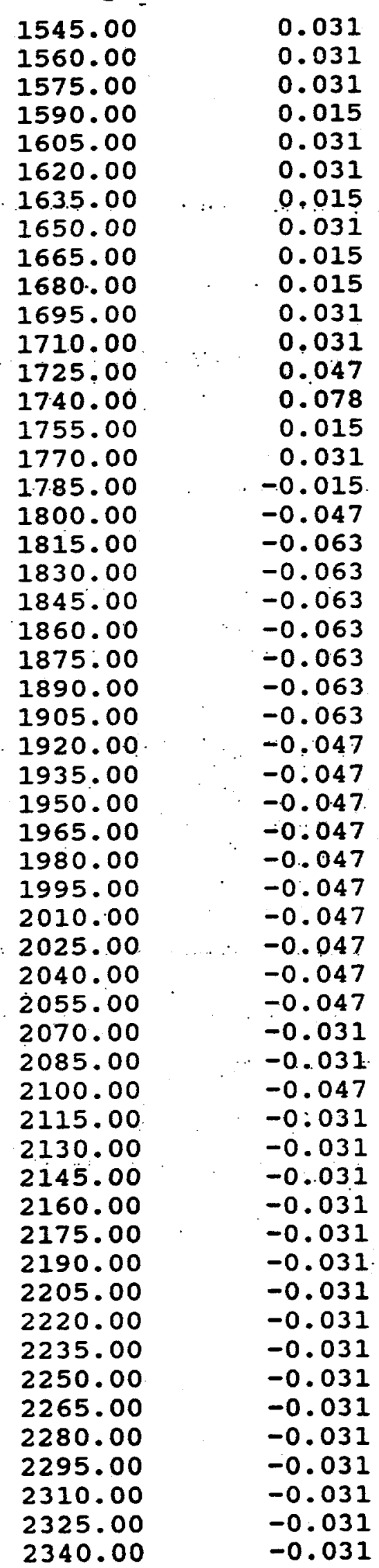




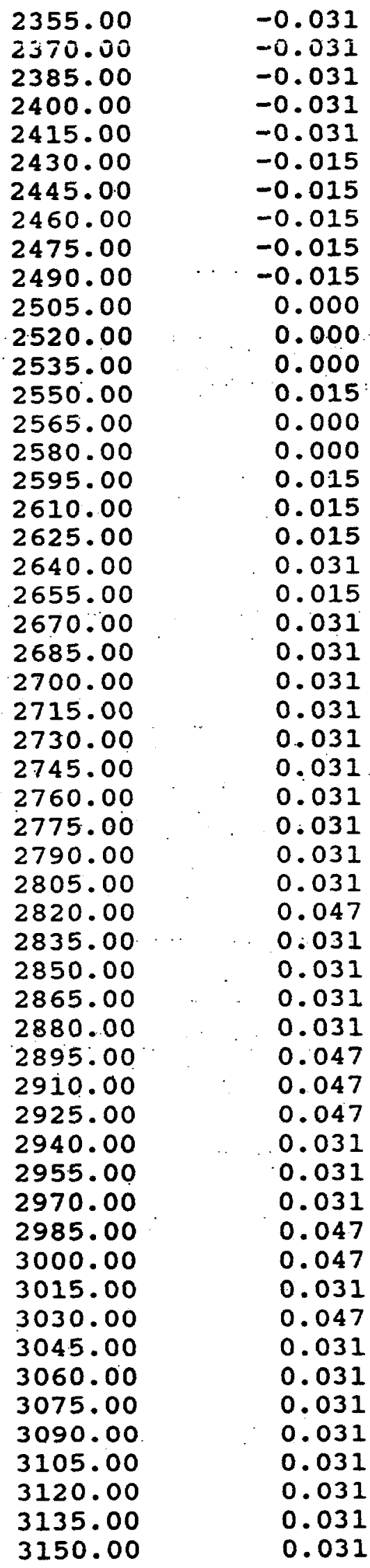




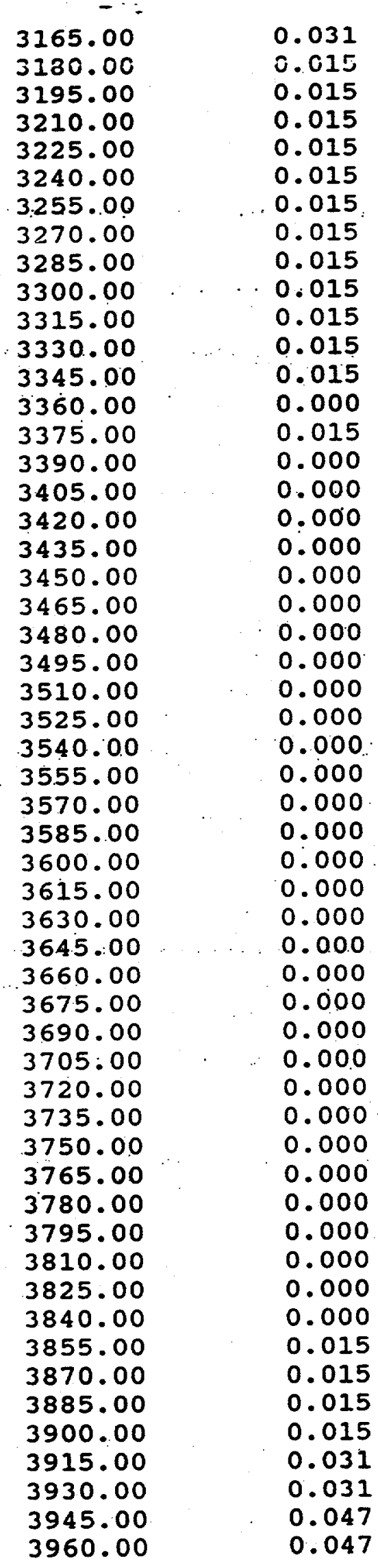


0.047 


\section{HSB 84D}

\section{APRII 27, 1990}

PRE-TEST NATER IEVEL CHANGES

EIAPSED TIME

CHANGE

0.0000

$-0.015$

15.0000

0.000

30.0000

45.0000

0.015

0.000

60.0000

0.000

75.0000

0.015

90.0000

0.015

1.05 .000

0.015

120.000

0.000

135.000

0.015

150.000

0.015

165.000

0.015

180.000

0.015

195.000

0.015

210.000

0.015

225.000

0.000

240.000

0.000

0.000

270.000

0.000

285.000

300.000

315.000

330.000

345.000

360.000

375.000

390.000

405.000

420.000

435.000

450.000

465.000

480.000

495.000

510.000

525.000

540.000

555.000

570.000

585.000

600.000

$-0.015$

$-0.015$

$-0.015$

$-0.015$

$-0.031$

$-0.015$

$-0.031$

$-0.031$

$-0.031$

$-0.031$

$-0.031$

$-0.031$

$-0.031$

$-0.031$

$-0.031$

$-0.031$

$-0.047$

$-0.047$

$-0.047$

$-0.047$

$-0.047$

$-0.047$

$-0.047$

615.000

$-0.047$

645.000

$-0.047$

660.000

$-0.0 .62$

675.000

$-0.062$

690.000

705.000

$-0.062$

$-0.062$

720.000

$-0.062$ 


$\begin{array}{ll}735.000 & \\ 750.000 & -0.062 \\ 765.000 & -0.062 \\ 780.000 & -0.062 \\ 795.000 & -0.062 \\ 810.000 & -0.062 \\ 825.000 & -0.062 \\ 840.000 & -0.062 \\ 855.000 & -0.062 \\ 870.000 & -0.062 \\ 885.000 & -0.062 \\ 900.000 & -0.062 \\ 915.000 & -0.062 \\ 930.000 & -0.062 \\ 945.000 & -0.062 \\ 960.000 & -0.062 \\ 975.000 & -0.062 \\ 990.000 & -0.062 \\ 1005.00 & -0.062 \\ 1020.00 & -0.062 \\ 1035.00 & -0.062 \\ 1050.00 & -0.062 \\ 1065.00 & -0.062 \\ 1080.00 & -0.047 \\ 1095.00 & -0.062 \\ 1110.00 & -0.062 \\ 1125.00 & -0.047 \\ 1140.00 & -0.062 \\ 1155.00 & -0.047 \\ 1170.00 & -0.047 \\ 1185.00 & -0.047 \\ 1200.00 & -0.047 \\ 1215.00 & -0.047 \\ 1230.00 & -0.062 \\ 1245.00 & -0.062 \\ 1260.00 & -0.062 \\ 1275.00 & -0.047 \\ 1290.00 & -0.047 \\ 1305.00 & -0.047 \\ 1320.00 & -0.062 \\ 1335.00 & -0.062 \\ 1350.00 & -0.078 \\ 1365.00 & -0.047 \\ 1380.00 & -0.062 \\ 1395.00 & -0.047 \\ 1410.00 & -0.047 \\ 1425.00 & -0.047 \\ 1440.00 & -0.047 \\ 1455.00 & -0.062 \\ 1470.00 & -0.062 \\ 1485.00 & -0.062 \\ 1500.00 & -0.047 \\ 1515.00 & -0.047 \\ 1530.00 & \end{array}$




$\begin{array}{ll}1545.00 & -0.047 \\ 1560.00 & -0.047 \\ 1575.00 & -0.062 \\ 1590.00 & -0.047 \\ 1605.00 & -0.047 \\ 1620.00 & -0.062 \\ 1635.00 & -0.047 \\ 1650.00 & -0.047 \\ 1665.00 & -0.047 \\ 1680.00 & -0.047 \\ 1695.00 & -0.047 \\ 1710.00 & -0.031 \\ 1725.00 & -0.047 \\ 1740.00 & -0.062 \\ 1755.00 & -0.094 \\ 1770.00 & -0.094 \\ 1785.00 & -0.110 \\ 1800.00 & -0.110 \\ 1815.00 & -0.125 \\ 1830.00 & -0.125 \\ 1845.00 & -0.110 \\ 1860.00 & -0.110 \\ 1875.00 & -0.110 \\ 1890.00 & -0.110 \\ 1905.00 & -0.110 \\ 1920.00 & -0.110 \\ 1935.00 & -0.110 \\ 1950.00 & -0.110 \\ 1955.00 & -0.125 \\ 1980.00 & -0.125 \\ 1995.00 & -0.110 \\ 2010.00 & -0.110 \\ 2025.00 & -0.110 \\ 2040.00 & -0.125 \\ 2055.00 & -0.110 \\ 2070.00 & -0.110 \\ 2085.00 & -0.125 \\ 2100.00 & -0.125 \\ 2115.00 & -0.125 \\ 2130.00 & -0.125 \\ 2145.00 & -0.125 \\ 2160.00 & -0.110 \\ 2175.00 & -0.125 \\ 2190.00 & -0.125 \\ 2205.00 & -0.110 \\ 2220.00 & -0.125 \\ 2235.00 & -0.125 \\ 2250.00 & -0.110 \\ 2265.00 & -0.110 \\ 2280.00 & -0.110 \\ 2295.00 & -0.110 \\ 2310.00 & -0.110 \\ 2340.00 & -110 \\ & \end{array}$




$\begin{array}{ll} & - \\ 2355.00 & \\ 2370.00 & -0.110 \\ 2385.00 & -0.110 \\ 2400.00 & -0.110 \\ 2415.00 & -0.110 \\ 2430.00 & -0.110 \\ 2445.00 & -0.110 \\ 2460.00 & -0.094 \\ 2475.00 & -0.094 \\ 2490.00 & -0.094 \\ 2505.00 & -0.078 \\ 2520.00 & -0.078 \\ 2535.00 & -0.078 \\ 2550.00 & -0.078 \\ 2565.00 & -0.062 \\ 2580.00 & -0.078 \\ 2595.00 & -0.078 \\ 2610.00 & -0.062 \\ 2625.00 & -0.062 \\ 2640.00 & -0.062 \\ 2655.00 & -0.047 \\ 2670.00 & -0.062 \\ 2685.00 & -0.047 \\ 2700.00 & -0.031 \\ 2715.00 & -0.047 \\ 2730.00 & -0.047 \\ 2745.00 & -0.047 \\ 2760.00 & -0.047 \\ 2775.00 & -0.047 \\ 2790.00 & -0.047 \\ 2805.00 & -0.047 \\ 2820.00 & -0.047 \\ 2835.00 & -0.031 \\ 2850.00 & -0.047 \\ 2865.00 & -0.047 \\ 2880.00 & -0.047 \\ 2895.00 & -0.047 \\ 2910.00 & -0.047 \\ 2925.00 & -0.047 \\ 2940.00 & -0.047 \\ 2955.00 & -0.047 \\ 2970.00 & -0.047 \\ 2985.00 & -0.047 \\ 3000.00 & -0.047 \\ 3015.00 & -0.047 \\ 3030.00 & -0.062 \\ 3045.00 & -0.047 \\ 3060.00 & -0.047 \\ 3075.00 & -0.047 \\ 3090.00 & -0.047 \\ 3105.00 & -0.047 \\ 3120.00 & -0.062 \\ 3135.00 & -0.062 \\ 3150.00 & \\ 2 & \end{array}$




$\begin{array}{ll}3165.00 & -0.062 \\ 2160.00 & -0.052 \\ 3195.00 & -0.062 \\ 3210.00 & -0.078 \\ 3225.00 & -0.062 \\ 3240.00 & -0.078 \\ 3255.00 & -0.062 \\ 3270.00 & -0.078 \\ 3285.00 & -0.078 \\ 3300.00 & -0.078 \\ 3315.00 & -0.078 \\ 3330.00 & -0.078 \\ 3345.00 & -0.078 \\ 3360.00 & -0.094 \\ 3375.00 & -0.094 \\ 3390.00 & -0.094 \\ 3405.00 & -0.078 \\ 3420.00 & -0.078 \\ 3435.00 & -0.094 \\ 3450.00 & -0.078 \\ 3465.00 & -0.078 \\ 3480.00 & -0.078 \\ 3495.00 & -0.078 \\ 3510.00 & -0.094 \\ 3525.00 & -0.094 \\ 3540.00 & -0.094 \\ 3555.00 & -0.094 \\ 3570.00 & -0.094 \\ 3585.00 & -0.094 \\ 3600.00 & -0.094 \\ 3615.00 & -0.094 \\ 3630.00 & -0.094 \\ 3645.00 & -0.094 \\ 3660.00 & -0.094 \\ 3675.00 & -0.094 \\ 3690.00 & -0.094 \\ 3705.00 & -0.09 .4 \\ 3720.00 & -0.094 \\ 3735.00 & -0.094 \\ 3750.00 & -0.094 \\ 3765.00 & -0.094 \\ 3780.00 & -0.094 \\ 3795.00 & -0.094 \\ 3810.00 & -0.094 \\ 3825.00 & -0.094 \\ 3840.00 & -0.078 \\ 3855.00 & -0.078 \\ 3870.00 & -0.078 \\ 3885.00 & -0.062 \\ 3900.00 & -0.062 \\ 3915.00 & -0.062 \\ 3930.00 & -0.047 \\ 3945.00 & -0.047 \\ 3960.00 & -0.047\end{array}$


$3975.00^{\circ} \quad-0.031$ 


\section{HBB 69}

\section{ARRII 27, 1990}

PRE-TEST WATER LEVEL CHANGES

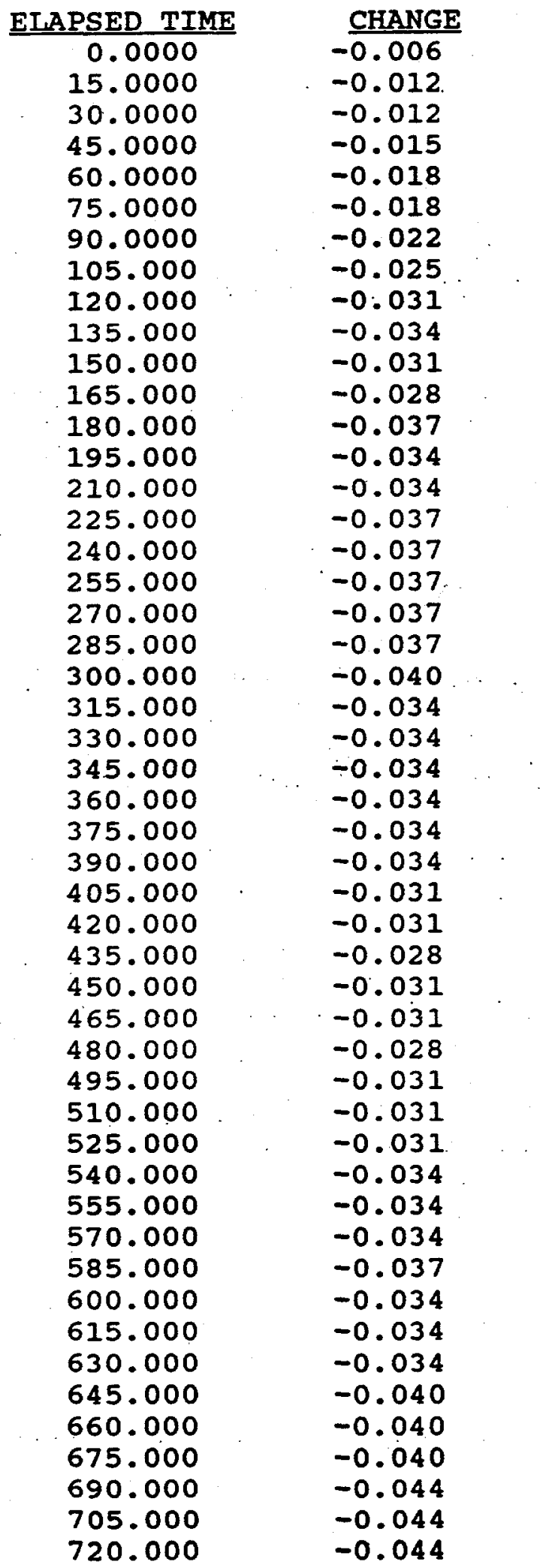




$\begin{array}{ll} & \\ 135.000 & \\ 750.000 & -0.044 \\ 765.000 & -0.047 \\ 780.000 & -0.044 \\ 795.000 & -0.044 \\ 810.000 & -0.044 \\ 825.000 & -0.044 \\ 840.000 & -0.044 \\ 855.000 & -0.047 \\ 870.000 & -0.044 \\ 885.000 & -0.040 \\ 900.000 & -0.040 \\ 915.000 & -0.037 \\ 930.000 & -0.037 \\ 945.000 & -0.037 \\ 960.000 & -0.034 \\ 975.000 & -0.034 \\ 990.000 & -0.034 \\ 1005.00 & -0.034 \\ 1020.00 & -0.037 \\ 1035.00 & -0.031 \\ 1050.00 & -0.037 \\ 1065.00 & -0.037 \\ 1080.00 & -0.031 \\ 1095.00 & -0.037 \\ 1110.00 & -0.040 \\ 1125.00 & -0.037 \\ 1140.00 & -0.037 \\ 1155.00 & -0.034 \\ 1170.00 & -0.037 \\ 1185.00 & -0.034 \\ 1200.00 & -0.034 \\ 1215.00 & -0.031 \\ 1230.00 & -0.044 \\ 1245.00 & -0.044 \\ 1260.00 & -0.044 \\ 1275.00 & -0.044 \\ 1290.00 & -0.037 \\ 1305.00 & -0.040 \\ 1320.00 & -0.056 \\ 1335.00 & -0.050 \\ 1350.00 & -0.063 \\ 1365.00 & -0.047 \\ 1380.00 & -0.053 \\ 1395.00 & -0.031 \\ 1410.00 & -0.044 \\ 1425.00 & -0.037 \\ 1440.00 & -0.047 \\ 1455.00 & -0.047 \\ 1470.00 & -0.053 \\ 1485.00 & -0.050 \\ 1500.00 & -0.050 \\ 1515.00 & -0.053 \\ 1530.00 & -0.047 \\ & -0.056\end{array}$




$\begin{array}{ll}1545.00 & -0.047 \\ 1500.00 & -0.040 \\ 1575.00 & -0.047 \\ 1590.00 & -0.044 \\ 1605.00 & -0.034 \\ 1620.00 & -0.050 \\ 1635.00 & -0.047 \\ 1650.00 & -0.047 \\ 1665.00 & -0.040 \\ 1680.00 & -0.034 \\ 1695.00 & -0.028 \\ 1710.00 & -0.015 \\ 1725.00 & -0.031 \\ 1740.00 & -0.040 \\ 1755.00 & -0.047 \\ 1770.00 & -0.047 \\ 1785.00 & -0.053 \\ 1800.00 & -0.056 \\ 1815.00 & -0.056 \\ 1830.00 & -0.053 \\ 1845.00 & -0.050 \\ 1860.00 & -0.044 \\ 1875.00 & -0.047 \\ 1890.00 & -0.044 \\ 1905.00 & -0.047 \\ 1920.00 & -0.050 \\ 1935.00 & -0.056 \\ 1950.00 & -0.056 \\ 1965.00 & -0.056 \\ 1980.00 & -0.059 \\ 1995.00 & -0.056 \\ 2010.00 & -0.053 \\ 2025.00 & -0.056 \\ 2040.00 & -0.053 \\ 2055.00 & -0.056 \\ 2070.00 & -0.056 \\ 2085.00 & -0.056 \\ 2100.00 & -0.059 \\ 2115.00 & -0.059 \\ 2130.00 & -0.059 \\ 2145.00 & -0.059 \\ 2160.00 & -0.056 \\ 2175.00 & -0.059 \\ 2190.00 & -0.056 \\ 2205.00 & -0.056 \\ 2220.00 & -0.059 \\ 2235.00 & -0.056 \\ 2250.00 & -0.053 \\ 2265.00 & -0.056 \\ 2280.00 & -0.053 \\ 2295.00 & -0.053 \\ 2310.00 & -0.050 \\ 2340.00 & -0.047 \\ 13200 & -0.050\end{array}$




$\begin{array}{ll}2355.00 & -0.050 \\ 2370.00 & -0.047 \\ 2385.00 & -0.044 \\ 2400.00 & -0.044 \\ 2415.00 & -0.044 \\ 2430.00 & -0.044 \\ 2445.00 & -0.040 \\ 2460.00 & -0.044 \\ 2475.00 & -0.037 \\ 2490.00 & -0.034 \\ 2505.00 & -0.025 \\ 2520.00 & -0.031 \\ 2535.00 & -0.034 \\ 2550.00 & -0.028 \\ 2565.00 & -0.034 \\ 2580.00 & -0.037 \\ 2595.00 & -0.031 \\ 2610.00 & -0.031 \\ 2625.00 & -0.022 \\ 2640.00 & -0.015 \\ 2655.00 & -0.028 \\ 2670.00 & -0.028 \\ 2685.00 & -0.018 \\ 2700.00 & -0.034 \\ 2715.00 & -0.040 \\ 2730.00 & -0.037 \\ 2745.00 & -0.031 \\ 2760.00 & -0.037 \\ 2775.00 & -0.037 \\ 2790.00 & -0.037 \\ 2805.00 & -0.040 \\ 2820.00 & -0.028 \\ 2835.00 & -0.047 \\ 2850.00 & -0.053 \\ 2865.00 & -0.050 \\ 2880.00 & -0.053 \\ 2895.00 & -0.047 \\ 2910.00 & -0.047 \\ 2925.00 & -0.047 \\ 2940.00 & -0.037 \\ 2955.00 & -0.056 \\ 2970.00 & -0.053 \\ 2985.00 & -0.053 \\ 3000.00 & -0.050 \\ 3015.00 & -0.063 \\ 3030.00 & -0.050 \\ 3045.00 & -0.056 \\ 3060.00 & -0.056 \\ 3075.00 & -0.056 \\ 3090.00 & -0.059 \\ 3105.00 & -0.063 \\ 3120.00 & -0.059 \\ 3135.00 & -0.063 \\ 3150.00 & -0.063\end{array}$




$\begin{array}{ll}3165.00 & -0.059 \\ 3180.00 & -0.059 \\ 3195.00 & -0.056 \\ 3210.00 & -0.056 \\ 3225.00 & -0.053 \\ 3240.00 & -0.053 \\ 3255.00 & -0.050 \\ 3270.00 & -0.053 \\ 3285.00 & -0.056 \\ 3300.00 & -0.053 \\ 3315.00 & -0.053 \\ 3330.00 & -0.056 \\ 3345.00 & -0.056 \\ 3360.00 & -0.059 \\ 3375.00 & -0.056 \\ 3390.00 & -0.059 \\ 3405.00 & -0.056 \\ 3420.00 & -0.056 \\ 3435.00 & -0.056 \\ 3450.00 & -0.053 \\ 3465.00 & -0.050 \\ 3480.00 & -0.050 \\ 3495.00 & -0.050 \\ 3510.00 & -0.050 \\ 3525.00 & -0.053 \\ 3540.00 & -0.053 \\ 3555.00 & -0.056 \\ 3570.00 & -0.056 \\ 3585.00 & -0.063 \\ 3600.00 & -0.059 \\ 3615.00 & -0.059 \\ 3630.00 & -0.063 \\ 3645.00 & -0.063 \\ 3660.00 & -0.059 \\ 3675.00 & -0.059 \\ 3690.00 & -0.056 \\ 3705.00 & -0.059 \\ 3720.00 & -0.056 \\ 3735.00 & -0.056 \\ 3750.00 & -0.053 \\ 3765.00 & -0.053 \\ 3780.00 & -0.053 \\ 3795.00 & -0.050 \\ 3810.00 & -0.050 \\ 3825.00 & -0.047 \\ 3840.00 & -0.044 \\ 3855.00 & -0.037 \\ 3870.00 & -0.037 \\ 3885.00 & -0.034 \\ 3900.00 & -0.031 \\ 3915.00 & -0.031 \\ 3930.00 & -028 \\ 3945.00 & \\ 3960.00 & \end{array}$


HSB 692

APRIL 27, 1990
PRE-TEST WATER LEVEL CHANGES

ELAPSED TIME

CHANGE

0.0000

15.975

15.0000

30.0000

15.956

45.0000

15.953

60.0000

15.947

75.0000

90.0000

105.000

120.000

135.000

150.000

15.937

15.937

15.934

15.928

15.925

15.922

165.000

15.922

180.000

15.922

195.000

15.919

210.000

15.915

225.000

15.915

15.915

240.000

15.912

255.000

15.912

270.000

285.000

300.000

315.000

330.000

345.000

15.912

15.912

15.912

15.915

15.915

360.000

15.915

375.000

390.000

405.000

420.000

435.000

450.000

465.000

480.000

495.000

510.000

525.000

540.000

555.000

570.000

585.000

600.000

15.915

15.915

15.915

15.915

15.915

15.919

15.915

15.919

15.919

15.919

15.919

15.919

15.915

15.919

15.919

15.919

15.919

15.919

615.000

15.919

645.000

15.915

660.000

15.915

675.000

15.915

690.000

15.915

705.000

15.915

720.000

15.915 


$\begin{array}{ll}735.000 & 15.915 \\ 750.000 & 15.915 \\ 765.000 & 15.915 \\ 780.000 & 15.915 \\ 795.000 & 15.915 \\ 810.000 & 15.915 \\ 825.000 & 15.915 \\ 840.000 & 15.912 \\ 855.000 & 15.912 \\ 870.000 & 15.915 \\ 885.000 & 15.912 \\ 900.000 & 15.915 \\ 915.000 & 15.912 \\ 930.000 & 15.915 \\ 945.000 & 15.915 \\ 960.000 & 15.915 \\ 975.000 & 15.915 \\ 990.000 & 15.915 \\ 1005.00 & 15.915 \\ 1020.00 & 15.919 \\ 1035.00 & 15.915 \\ 1050.00 & 15.915 \\ 1065.00 & 15.919 \\ 1080.00 & 15.919 \\ 1095.00 & 15.915 \\ 1110.00 & 15.915 \\ 1125.00 & 15.915 \\ 1140.00 & 15.915 \\ 1155.00 & 15.915 \\ 1170.00 & 15.915 \\ 1185.00 & 15.919 \\ 1200.00 & 15.922 \\ 1215.00 & 15.915 \\ 1230.00 & 15.912 \\ 1245.00 & 15.915 \\ 1260.00 & 15.915 \\ 1275.00 & 15.919 \\ 1290.00 & 15.922 \\ 14305.00 & 15.906 \\ 1320.00 & 15.909 \\ 1515.00 & 15.906 \\ 1530.00 & 15.912 \\ 1350.00 & 15.909 \\ 1365.00 & 15.925 \\ 1380.00 & 15.915 \\ 1395.00 & 15.919 \\ 1410.00 & 15.915 \\ 1425.00 & 15.909 \\ 1440.00 & 15.903 \\ 1455.00 & 15.900 \\ 1470.900 & \end{array}$




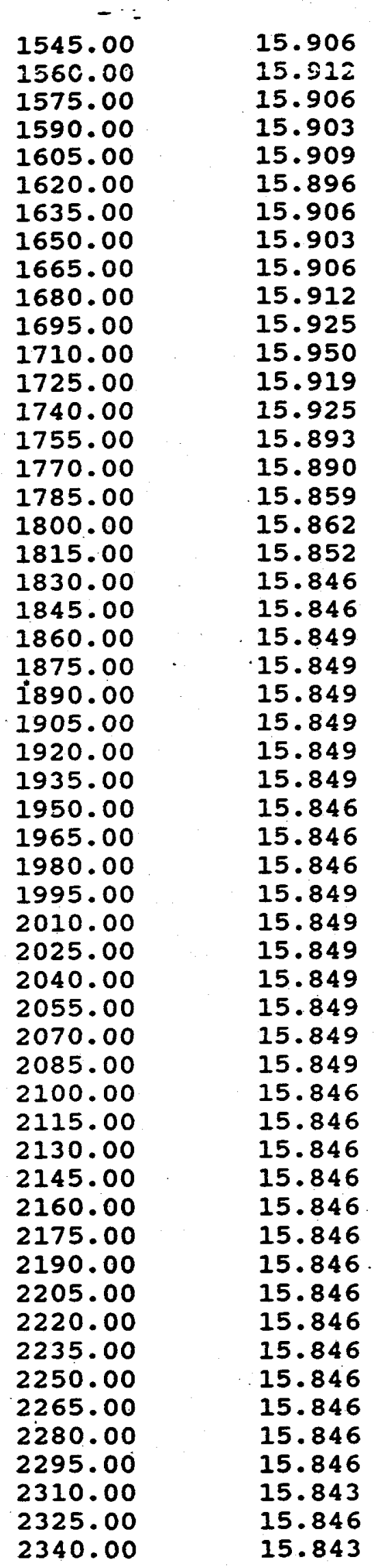




$\begin{array}{ll} & \\ 2355.00 & 15.846 \\ 2370.00 & 15.843 \\ 2385.00 & 15.846 \\ 2400.00 & 15.846 \\ 2415.00 & 15.846 \\ 2430.00 & 15.849 \\ 2445.00 & 15.852 \\ 2460.00 & 15.849 \\ 2475.00 & 15.852 \\ 2490.00 & 15.852 \\ 2505.00 & 15.862 \\ 2520.00 & 15.855 \\ 2535.00 & 15.849 \\ 2550.00 & 15.859 \\ 2565.00 & 15.852 \\ 2580.00 & 15.849 \\ 2595.00 & 15.852 \\ 2610.00 & 15.855 \\ 2625.00 & 15.865 \\ 2640.00 & 15.865 \\ 2655.00 & 15.859 \\ 2670.00 & 15.859 \\ 2685.00 & 15.868 \\ 2700.00 & 15.855 \\ 2715.00 & 15.852 \\ 2730.00 & 15.855 \\ 2745.00 & 15.859 \\ 2760.00 & 15.855 \\ 2775.00 & 15.855 \\ 2790.00 & 15.852 \\ 2805.00 & 15.852 \\ 2820.00 & 15.862 \\ 2835.00 & 15.846 \\ 2850.00 & 15.843 \\ 2865.00 & 15.843 \\ 2880.00 & 15.840 \\ 2895.00 & 15.846 \\ 2910.00 & 15.846 \\ 2925.00 & 15.849 \\ 2940.00 & 15.859 \\ 2955.00 & 15.843 \\ 2970.00 & 15.840 \\ 2985.00 & 15.836 \\ 3000.00 & 15.840 \\ 3015.00 & 15.833 \\ 3030.00 & 15.840 \\ 3045.00 & 15.836 \\ 3060.00 & 15.836 \\ 3075.00 & 15.833 \\ 3090.00 & 15.830 \\ 3120.00 & 15.830 \\ 3150.00 & 15.827 \\ 2530 & \end{array}$




$\begin{array}{ll}3165.00 & \\ 3180.00 & 15.827 \\ 3195.00 & 15.830 \\ 3210.00 & 15.830 \\ 3225.00 & 15.830 \\ 3240.00 & 15.830 \\ 3255.00 & 15.830 \\ 3270.00 & 15.830 \\ 3285.00 & 15.827 \\ 3300.00 & 15.827 \\ 3315.00 & 15.827 \\ 3330.00 & 15.827 \\ 3345.00 & 15.824 \\ 3360.00 & 15.824 \\ 3375.00 & 15.824 \\ 3390.00 & 15.824 \\ 3405.00 & 15.824 \\ 3420.00 & 15.824 \\ 3435.00 & 15.824 \\ 3450.00 & 15.824 \\ 3465.00 & 15.821 \\ 3480.00 & 15.824 \\ 3495.00 & 15.824 \\ 3510.00 & 15.821 \\ 3525.00 & 15.821 \\ 3540.00 & 15.821 \\ 3555.00 & 15.821 \\ 3570.00 & 15.818 \\ 3585.00 & 15.818 \\ 3600.00 & 15.814 \\ 3615.00 & 15.814 \\ 3630.00 & 15.814 \\ 3645.00 & 15.814 \\ 3660.00 & 15.814 \\ 3675.00 & 15.814 \\ 3690.00 & 15.814 \\ 3705.00 & 15.814 \\ 3720.00 & 15.814 \\ 3735.00 & 15.814 \\ 3750.00 & 15.814 \\ 3765.00 & 15.814 \\ 3780.00 & 15.814 \\ 3795.00 & 15.818 \\ 3810.00 & 15.818 \\ 3825.00 & 15.818 \\ 3840.00 & 15.821 \\ 3855.00 & 15.824 \\ 3870.00 & 15.827 \\ 3885.00 & 15.827 \\ 3900.00 & 15.827 \\ 3915.00 & 15.827 \\ 3930.00 & 15.830 \\ 3945.00 & 15.830\end{array}$


